\title{
THESIS
}

\section{A MULTI-FUNCTIONAL ELECTROLYTE FOR LITHIUM-ION BATTERIES}

\author{
Submitted by \\ Kevin A. Westhoff \\ Department of Mechanical Engineering
}

In partial fulfillment of the requirements

For the Degree of Master of Science

Colorado State University

Fort Collins, Colorado

Summer 2016

Master's Committee:

Advisor: Todd M. Bandhauer

Thomas H. Bradley

Amy L. Prieto 
Copyright by Kevin A. Westhoff 2016

All Rights Reserved 


\section{ABSTRACT}

\section{A MULTI-FUNCTIONAL ELECTROLYTE FOR LITHIUM-ION BATTERIES}

Thermal management of lithium-ion batteries (LIBs) is paramount for multi-cell packs, such as those found in electric vehicles, to ensure safe and sustainable operation. Thermal management systems (TMSs) maintain cell temperatures well below those associated with capacity fade and thermal runaway to ensure safe operation and prolong the useful life of the pack. Current TMSs employ single-phase liquid cooling to the exterior surfaces of every cell, decreasing the volumetric and gravimetric energy density of the pack. In the present study, a novel, internal TMS that utilizes a multi-functional electrolyte (MFE) is investigated, which contains a volatile co-solvent that boils upon heat absorption in small channels in the positive electrode of the cell.

The inert fluid HFE-7000 is investigated as the volatile co-solvent in the MFE (1 M LiTFSI

in 1:1 HFE-7000/ethyl methyl carbonate by volume) for the proposed TMS. In the first phase of the study, the baseline electrochemical performance of the MFE is determined by conductivity, electrochemical stability window, half and full cell cycling with lithium iron phosphate $\left(\mathrm{LiFePO}_{4}\right)$, lithium titanate oxide $\left(\mathrm{Li}_{4} \mathrm{Ti}_{5} \mathrm{O}_{12}\right)$, and copper antimonide $\left(\mathrm{Cu}_{2} \mathrm{Sb}\right)$, and impedance spectroscopy measurements. The results show that the MFE containing HFE-7000 has comparable stability and cycling performance to a conventional lithium-ion electrolyte (1 $\mathrm{M} \mathrm{LiPF} 6$ in 3:7 ethylene carbonate/diethyl carbonate by weight). The MFE-containing cells had higher impedance than carbonate-only cells, indicating reduced passivation capability on the electrodes. Additional investigation is warranted to refine the binary MFE mixture by the addition of solid electrolyte interphase (SEI) stabilizing additives. 
To validate the thermal and electrochemical performance of the $\mathrm{MFE}, \mathrm{Cu}_{2} \mathrm{Sb}$ and $\mathrm{LiFePO}_{4}$ are used in a full cell architecture with the MFE in a custom electrolyte boiling facility. The facility enables direct viewing of the vapor generation within the channel in the positive electrode and characterizes the galvanostatic electrochemical performance. Test results show that the $\mathrm{LiFePO}_{4} / \mathrm{Cu}_{2} \mathrm{Sb}$ cell is capable of operation even when a portion of the more volatile $\mathrm{HFE}-7000$ is continuously evaporated under an extreme heat flux, proving the concept of a MFE. The conclusions presented in this work inform the future development of the proposed internal TMS. 


\section{ACKNOWLEDGEMENTS}

I would first like to thank my advisor, Dr. Todd Bandhauer, for his instrumental role in my graduate education. My time as a graduate student was supported entirely by the technical forethought of Dr. Bandhauer. I greatly appreciate the projects I was able to work on, especially their direct relevancy to current industry. It has been an incredible learning experience helping to create and working within the Interdisciplinary Thermal Science Lab. It is difficult to quantify how much I have grown technically and professionally from my interactions with Dr. Bandhauer over the past couple of years. Also, thank you for your thorough feedback on this document.

I know my research significantly benefited from my interactions with CSU's Chemistry department. Specifically, I would like to thank Everett Jackson and Dr. Amy Prieto for their extremely useful discussions on the electrochemical aspects of my research. Their unparalleled expertise truly helped to bring me up to speed on a completely foreign subject. In addition, I would like to thank Dr. Wesley Hoffert at Prieto Battery for his help in preparing electrodes used in this study as well as technical discussions on my research. I would also like to recognize Dr. Anita Kear and Dr. James Dong at Prieto Battery for their assistance on my project.

Fellow ITS Lab members also deserve recognition for their support of my research, specifically in their useful discussions around the design, fabrication, and troubleshooting of my test facility. I would like to directly thank Torben Grumstrup, Taylor Bevis, Jonas Adler, and Trevor Vernon.

Finally I would like to thank my friends and family who kept me grounded throughout this experience. Amy, thank you for all the delicious meals and constant reminders that there is light at the end of the tunnel, I love you. Tyler Schott and Darryl Beemer, I am looking forward to our future canoe adventures outside of CSU. 


\section{TABLE OF CONTENTS}

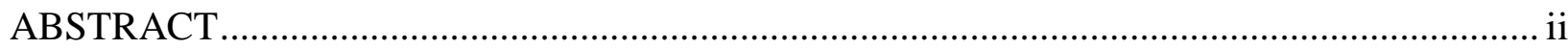

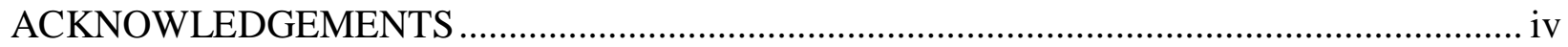

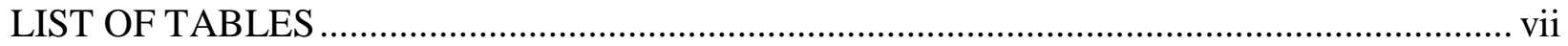

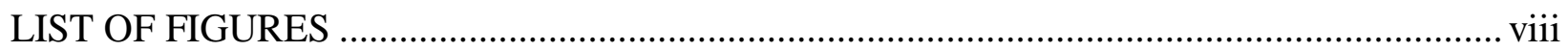

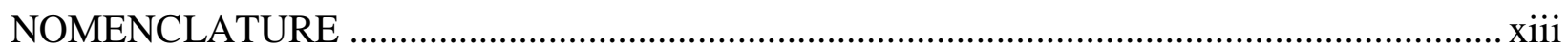

CHAPTER 1. INTRODUCTION ……………........................................................... 1

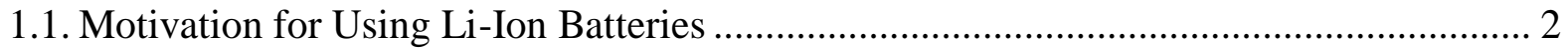

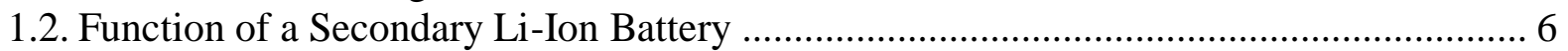

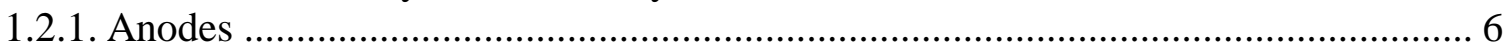

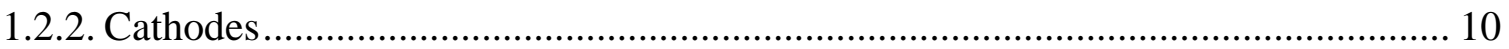

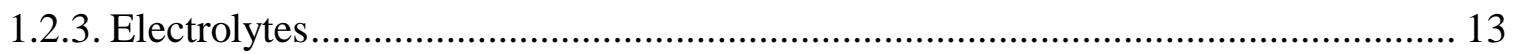

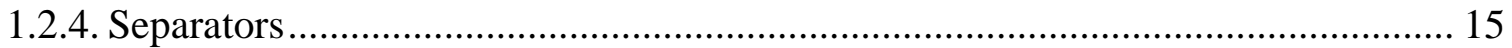

1.2.5. Li-Ion Battery Function ....................................................................................... 16

1.2.6. Li-Ion Battery Heat Generation During Normal Operation ................................... 17

1.3. Fundamental Thermal Limitations of Li-Ion Batteries ................................................... 20

1.3.1. Thermal Runaway ......................................................................................... 22

1.3.2. High Profile Li-Ion Battery Failures ……………………………………….... 22

1.4. Thesis Organization ……………………………................................................ 27

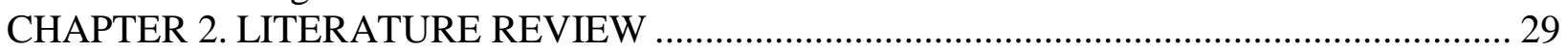

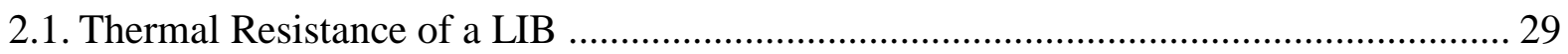

2.2. State-of-the-Art Thermal Management Systems for Large LIB Packs in EVs................ 36

2.3. Internal Thermal Management of LIBs........................................................................ 43

2.4. Prior LIB Electrolyte Modification Research ……….................................................. 46

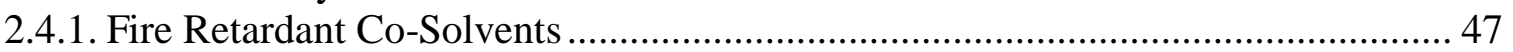

2.4.2. Non-Flammable Fluids as Co-Solvents ............................................................. 52

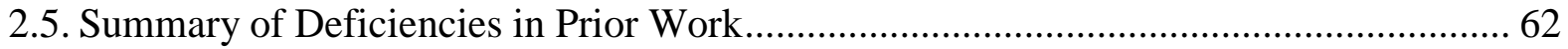

2.6. Focus of Current Investigation ................................................................................... 64

CHAPTER 3. CONTINUOUS, PASSIVE INTERNAL COOLING WITH A MULTI-

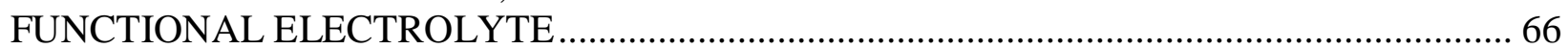

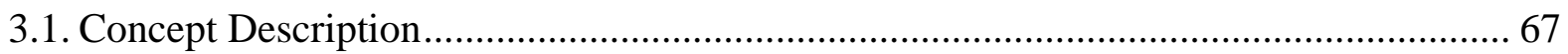

3.1.1. Impact of the Proposed Internal TMS ……………........................................... 71

3.2. Multi-Functional Electrolyte Requirements.................................................................... 74

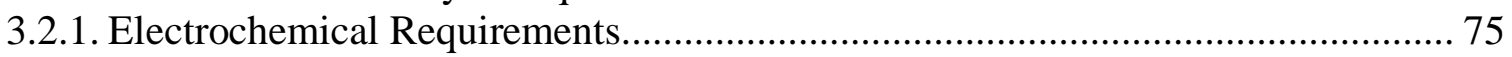

3.2.2. Thermal Requirements ................................................................................ 82

3.3. Components of the Multi-Functional Electrolyte …………………………….............. 84

3.3.1. Candidate Volatile Co-Solvents ....................................................................... 85

3.3.2. Candidate Organic Carbonate Co-Solvents and Lithium Salt ................................ 88

3.3.3. Baseline Electrolyte for Multi-Functional Electrolyte Performance Comparison.. 90

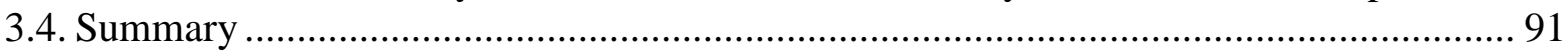

CHAPTER 4. NON-BOILING ELECTROCHEMICAL PERFORMANCE OF THE MULTI-

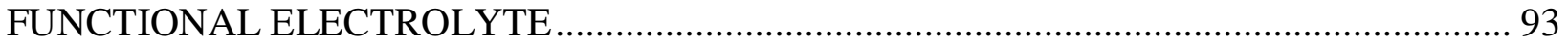

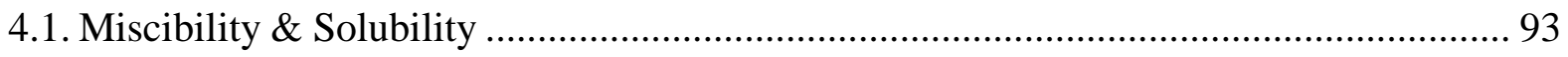

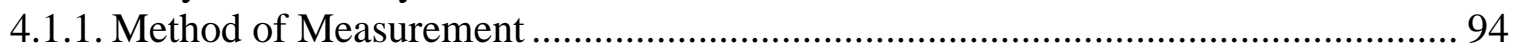




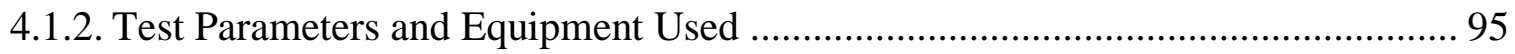

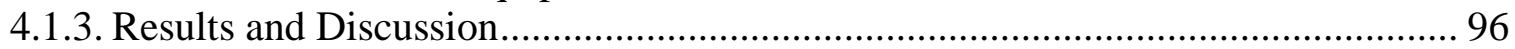

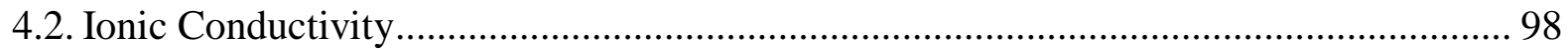

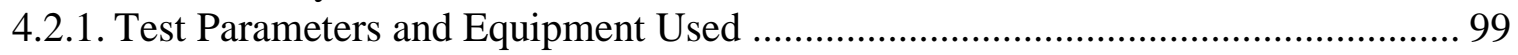

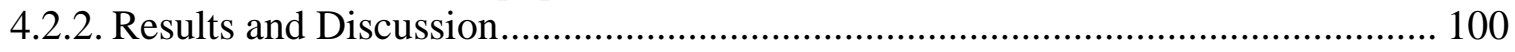

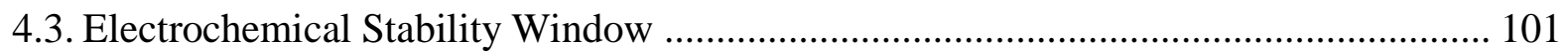

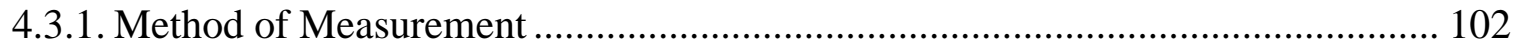

4.3.2. Test Parameters and Equipment Used ......................................................... 106

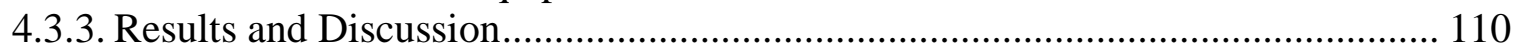

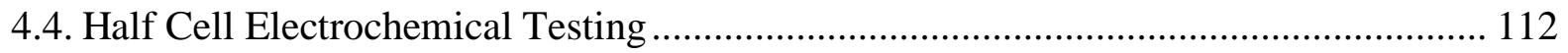

4.4.1. Definition and Method of Measurement ..................................................... 112

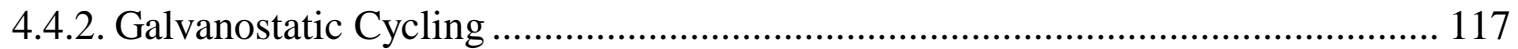

4.4.3. Electrochemical Impedance Spectroscopy.................................................... 119

4.4.4. Test Parameters and Equipment Used .......................................................... 136

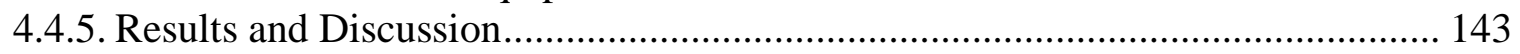

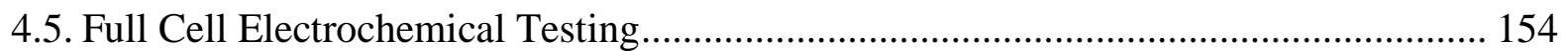

4.5.1. Definition and Method of Measurement ........................................................... 154

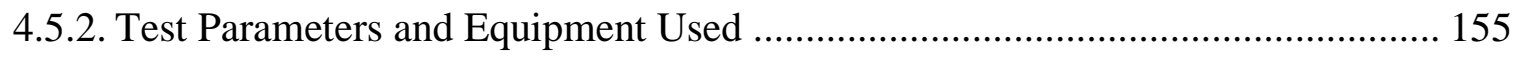

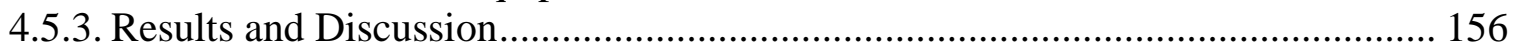

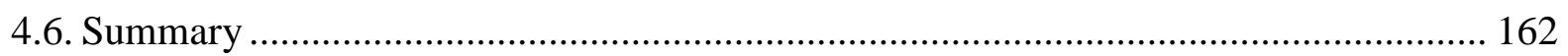

CHAPTER 5. ELECTROCHEMICAL PERFORMANCE OF MULTI-FUNCTIONAL ELECTROLYTE WITH SIMULTANEOUS CO-SOLVENT BOILING .............................. 164

5.1. Experimental Requirements for Custom Electrolyte Boiling Facility ........................ 164

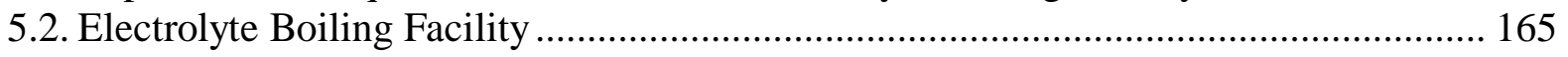

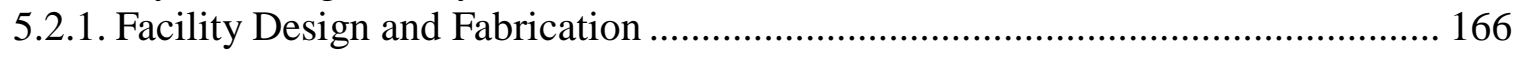

5.2.2. Cleaning of Electrolyte Wetted Components................................................. 172

5.2.3. Assembly of Electrolyte Boiling Facility....................................................... 173

5.2.4. Charging Electrolyte Boiling Facility with Multi-Functional Electrolyte ............ 178

5.3. Test Parameters and Equipment Used ................................................................ 181

5.3.1. Data Acquisition and Auxiliary Components ............................................. 182

5.3.2. Thermal Control of Electrolyte Boiling Facility ......................................... 185

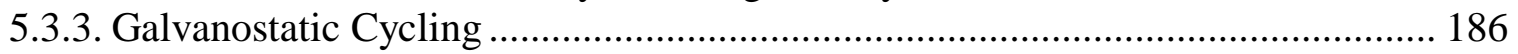

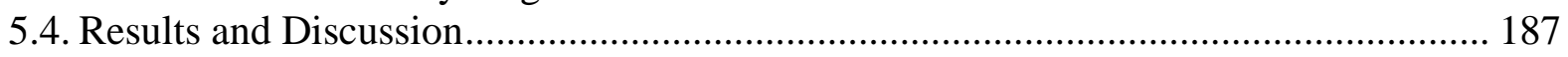

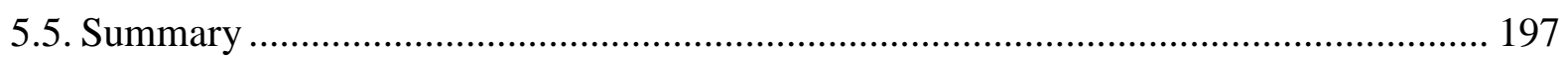

CHAPTER 6. CONCLUSION AND RECOMMENDATIONS ......................................... 199

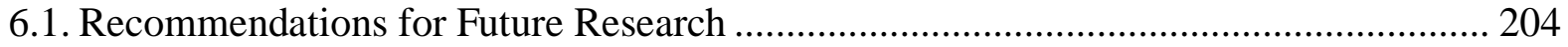

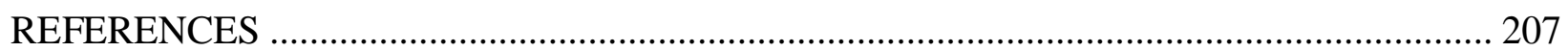

APPENDIX A. FREEZE-PUMP-THAW LIQUID DEGASSING PROCEDURE ................. 215

APPENDIX B. WORKING ELECTRODE POLISHING PROCEDURE............................... 218

APPENDIX C. SLURRY-BASED ELECTRODE COATING PROCEDURE ....................... 220

APPENDIX D. ELECTROLYTE BOILING FACILTIY COMPONENT LIST ..................... 226

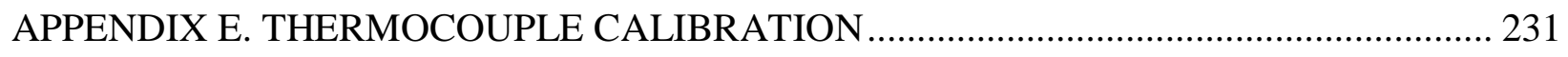




\section{LIST OF TABLES}

Table 1-1: $\quad$ Physical Properties of Some Commonly Applied Cathode Active Materials ...... 12

Table 2-1: $\quad$ Thermal Conduction Resistance for Chevrolet Volt 15 Ah Cell ........................ 34

Table 2-2: $\quad$ Thermal Conduction Resistance for Boeing 75 Ah Cell .................................. 35

Table 2-3: $\quad$ Summary of LIB Packs in Vehicles........................................................... 42

Table 2-4: $\quad$ Summary of Phosphorus-Based Fire Retardant Co-Solvents in LIB Electrolytes 51

Table 2-5: $\quad$ Summary of Non-Flammable Fluids as Co-Solvents in LIB Electrolytes............ 60

Table 3-1: $\quad$ Values Used for System Impact of Proposed Internal TMS ............................ 71

Table 3-2: $\quad$ System Impact of Proposed Internal TMS Compared to Chevrolet Volt ............. 73

Table 3-3: $\quad$ Summary of Current and Proposed TMSs for LIBs ....................................... 74

Table 3-4: Candidate Volatile Co-Solvents for the MFE ................................................. 86

Table 3-5: $\quad$ Common Organic Carbonate Solvents in LIB Liquid Electrolytes ..................... 89

Table 4-1: $\quad$ Suppliers for Components of Multi-Functional Electrolyte ............................... 94

Table 4-2: $\quad$ Room Temperature Ionic Conductivity of Investigated Electrolytes ................ 100

Table 4-3: $\quad$ Impedance of Common Circuit Elements used for EIS Measurements ............. 131

Table 4-4: $\quad$ Slurry Composition for LFP and LTO Electrodes by Weight Percent ............... 137

Table 4-5: $\quad$ Half Cell Voltage Limits and Capacity ............................................................ 141

Table 4-6: Generic Half Cell Test Schedule for Galvanostatic Cycling Tests .................... 141

Table 4-7: $\quad$ EIS Experimental Sequence Defined in Sequence Wizard of Gamry Framework

Table 4-8: $\quad$ Average EIS Fit Parameters for Half Cells, All Potentials are vs. $\mathrm{Li} / \mathrm{Li}^{+}$

Table 4-9: $\quad$ Full Cell Voltage Limits and Capacity ............................................................. 156

Table 4-10: $\quad$ Average EIS Fit Parameters for Full Cells ................................................... 161

Table 5-1: $\quad$ Requirements of the Electrolyte Boiling Facility .......................................... 165

Table 5-2: $\quad$ Testing Procedure for Electrolyte Boiling Facility ........................................ 187

Table D-1: $\quad$ Components of Electrolyte Boiling Facility ................................................. 226

Table E-1: $\quad$ Thermocouple Calibration Fits and Total Uncertainty .................................... 234 


\section{LIST OF FIGURES}

Figure 1-1: LIBs Compared to NiMH and Ni-Cd (Size and Weight Values are for $640 \mathrm{Wh}$ battery) .................................................................................................... 2

Figure 1-2: US Solar and Wind Energy Consumption [9]................................................... 5

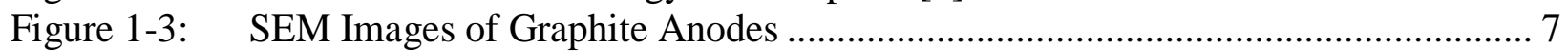

Figure 1-4: Lithiation and Delithation of Graphite at $\mathrm{C} / 30$ Rate, Voltage vs. $\mathrm{Li} / \mathrm{Li}^{+}$................. 8

Figure 1-5: SEM Images of Cathode Active Material Particles ............................................... 10

Figure 1-6: Lithiation of Cathode Active Materials at C/30 Rate, Voltage vs. $\mathrm{Li}^{2} / \mathrm{Li}^{+}$............. 12

Figure 1-7: Planar SEM Image of Microporous PP Separator ............................................ 15

Figure 1-8: Schematic of a LIB During Discharge [24] ....................................................... 16

Figure 1-9: Boeing APU Thermal Runaway (Left: Fire from APU; Middle: New APU; Right: Failed APU) [35] .................................................................................... 23

Figure 1-10: Chevrolet Volt Event Series (Left: Side-Impact Test, Middle: LIB Pack Damage, Right: Post Thermal Runaway Event) [38] ...................................................... 25

Figure 2-1: Tesla 85 kWh External TMS, Adapted from [48] …………………................. 30

Figure 2-2: Chevrolet Volt 15 Ah LIB ..................................................................... 31

Figure 2-3: Repeating Unit Cell for Calculation of Thermal Resistance of a 15 Ah LIB ....... 33

Figure 2-4: Boeing APU 75 Ah Cell Construction Schematic, Adapted from [35] ................. 34

Figure 2-5: Nissan LEAF 24 kWh LIB Pack [49] ............................................................... 36

Figure 2-6: Left: Tesla Model S with 85 kWh LIB Pack (Bottom of Image, Source: Tesla.com)

Right: Uncovered Pack [48]......................................................................... 38

Figure 2-7: Unwound Coolant Conduit from the 18650 Cells of a Tesla 85 kWh LIB Pack [48]

Figure 2-8: $\quad$ Aluminum Cooling Plates Used in Chevrolet Volt Battery Pack [63] .................. 40

Figure 2-9: 2016 Chevrolet Volt 18.4 kWh LIB Pack (Source: Chevrolet.com) ..................... 41

Figure 2-10: Bandhauer et al. Proposed Internal TMS with Microchannel Evaporator Containing R-134a Refrigerant [72] ................................................................................. 44

Figure 3-1: Schematic of Two-Phase Closed Loop Thermosiphon [93] ................................. 67

Figure 3-2: Proposed Internal Thermal Management System for Lithium-Ion Battery Using

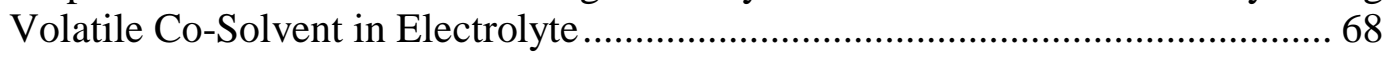

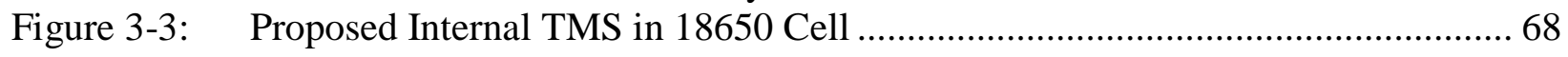

Figure 3-4: Representative Vapor Generation Channel Created in a Positive Electrode ........ 69

Figure 3-5: Embodiment 1 of Proposed Internal TMS with External Liquid Cooled Condenser on Edge Face of LIB Cells .............................................................................. 70

Figure 3-6: Embodiment 2 of Proposed Internal TMS with External Air Cooled Condenser on Large Face of LIB Cells.................................................................................. 70

Figure 3-7: Internal Short Caused by Nail Penetration of a $\mathrm{LiCoO}_{2} / \mathrm{Graphite}$ Cell at $4.2 \mathrm{~V}$ [20]

Figure 3-8: $\quad$ Open-Circuit Energy Diagram for a Lithium-Ion Electrolyte [19].......................... 77

Figure 3-9: Ethylene Carbonate Coordination Sphere around a Positively Charge Lithium-Ion (Oxygen —red; carbon—green; lithium — blue) [22] ........................................ 79

Figure 3-10: P-h Diagram of HFE-7000 with a $34^{\circ} \mathrm{C}$ Isotherm ............................................ 83

Figure 3-11: P-h Diagram of HFE-7100 Showing Multiple Lines of Constant Temperature ... 86

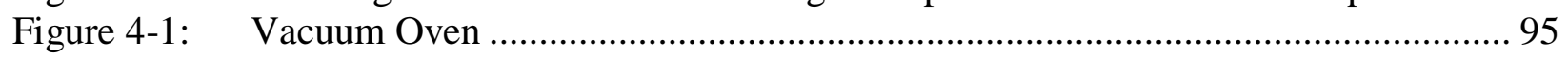


Figure 4-2: $\quad$ Separated Mixtures of EMC/Perflenapent and EMC/FC-72 …........................ 97

Figure 4-3: $\quad$ Unsolvated LiTFSI Salt (Left) and MFE Solution (1 M LiTFSI in 1:1 HFE7000/EMC by Volume) on Stir Plate (Right) ................................................... 98

Figure 4-4: $\quad$ Modified Glass Jar Lid for Conductivity Probe (Left) and Conductivity Probe Submersion in Electrolyte (Right) .......................................................... 99

Figure 4-5: $\quad$ LUMO and HOMO of HFE-7100 (labeled as MFE) Compared to Other Carbonate Solvents [90]

Figure 4-6: 3-Electrode Experimental Setup for CV Experiment ................................... 102

Figure 4-7: $\quad$ Effect of Potential Scan Rate, $v$ in $\mathrm{mV} \mathrm{s}^{-1}$, on Electrochemical Stability Window of E1 Measured with a GCE Working, Lithium Metal Counter and Reference

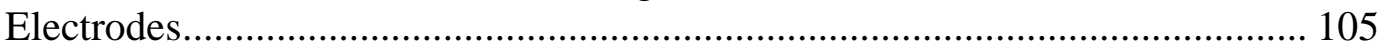

Figure 4-8: Gamry Reference 3000 Potentiostat ...................................................... 106

Figure 4-9: Gamry Calibration Circuit and Faraday Cage (source: gamry.com) ................. 107

Figure 4-10: Glassy Carbon and Platinum Working Electrodes Used for Electrochemical Stability Window Measurements ................................................................ 107

Figure 4-11: Electrochemical Stability Window Experimental Setup with Modified Glass Jar Lid

Figure 4-12: Gamry Reference 300 Potentiostat Leads ................................................. 108

Figure 4-13: Applied Potential Scan and Measured Current during Electrochemical Stability Window Measurement of E1 at $10 \mathrm{mV} \mathrm{s}^{-1}$ with a Glassy Carbon Working Electrode 109

Figure 4-14: Electrochemical Stability Window Measured with a GCE WE, Li metal CE/RE, 10 $\mathrm{mV} \mathrm{s}^{-1}$ Scan Rate, $0.5 \mathrm{~V}$ and $5 \mathrm{~V}$ vs. $\mathrm{Li} / \mathrm{Li}^{+}$Switching Potentials .................... 110

Figure 4-15: Electrochemical Stability Window Measured with a Pt WE, Li metal CE/RE, 10 $\mathrm{mV} \mathrm{s}^{-1}$ Scan Rate, $0.5 \mathrm{~V}$ and $5 \mathrm{~V}$ vs. Li/Li ${ }^{+}$Switching Potentials..................... 111

Figure 4-16: Half Cell Stacking Architecture Performed in Argon Glove Box .................... 116

Figure 4-17: Assembled LFP Half Cell .......................................................................... 117

Figure 4-18: Impedance Spectra of a Randles Cell from $1 \mathrm{~Hz}-5 \mathrm{kHz}$............................ 126

Figure 4-19: Electrical Circuit Schematic of Randles Cell............................................... 126

Figure 4-20: $\quad$ Nyquist Plot of Impedance Data from Randles Cell from $1 \mathrm{~Hz}-5 \mathrm{kHz} . \ldots \ldots \ldots . . .127$

Figure 4-21: $\quad$ Bode Plot of Impedance Data from Randles Cell from $1 \mathrm{~Hz}-5 \mathrm{kHz} \ldots \ldots \ldots \ldots \ldots . . . . .128$

Figure 4-22: $\quad$ Nyquist Plot of Impedance of $R$-CPE Circuit with Varying $\alpha$ [123] ................ 129

Figure 4-23: $\quad$ Nyquist Plot of $200 \Omega$ Resistor Impedance from $1 \mathrm{~Hz}-5 \mathrm{kHz}$..................... 130

Figure 4-24: Nyquist Plot of $1 \mu \mathrm{F}$ Capacitor Impedance from $1 \mathrm{~Hz}-5 \mathrm{kHz}$..................... 131

Figure 4-25: Equivalent Circuit Model Used for Impedance Fitting in Half and Full Cell Tests

Figure 4-26: Example of Impedance Model Fitting to Measured Data for $\mathrm{Cu}_{2} \mathrm{Sb} / \mathrm{Li} \mathrm{Cell} \mathrm{at} 0.5 \mathrm{~V}$ vs. $\mathrm{Li} / \mathrm{Li}^{+}$from $0.1 \mathrm{~Hz}-100 \mathrm{kHz}$.............................................................. 134

Figure 4-27: Frequency-Dependent Preferential Current Paths Through Equivalent Circuit . 135

Figure 4-28: $\quad \mathrm{Cu}_{2} \mathrm{Sb}$ Electrodeposition Setup at Prieto Battery ............................................. 137

Figure 4-29: Post-Electrodeposition of $3 \mu \mathrm{m}$ of $\mathrm{Cu}_{2} \mathrm{Sb}$ onto $15 \mu \mathrm{m}$ Copper Foil .................. 138

Figure 4-30: Arbin BT-2143 Battery Tester (Left) and Tenney Environmental Chamber (Right)

Figure 4-31: Arbin Battery Tester Lead Connections to Full Cells in Environmental Chamber 139 
Figure 4-32: Lithiation and Delithiation Capacity and Coulombic Efficiency of LTO/Li Cells Figure 4-33: (a) Nyquist Plot at $1 \mathrm{~V}$ vs. $\mathrm{Li} / \mathrm{Li}^{+}$After 10 and 20 Cycles (b) Nyquist Plot at $2 \mathrm{~V}$ vs $\mathrm{Li} / \mathrm{Li}^{+}$After 10 and 20 Cycles for LTO/Li Cells ............................................ 145

Figure 4-34: LTO/Li Cell Voltage vs. State of Lithiation (a) $10^{\text {th }}$ Cycle (b) $20^{\text {th }}$ Cycle ......... 146 Figure 4-35: Lithiation and Delithiation Capacity and Coulombic Efficiency of $\mathrm{Cu}_{2} \mathrm{Sb} / \mathrm{Li}$ Cells

Figure 4-36: (a) Nyquist Plot at $0.5 \mathrm{~V}$ vs. $\mathrm{Li} / \mathrm{Li}^{+}$After 10 and 20 Cycles (b) Nyquist Plot at 0.95 $\mathrm{V}$ vs. $\mathrm{Li} / \mathrm{Li}^{+}$After 10 and 20 Cycles for $\mathrm{Cu}_{2} \mathrm{Sb} / \mathrm{Li}$ Cells ................................. 148

Figure 4-37: $\quad \mathrm{Cu}_{2} \mathrm{Sb} / \mathrm{Li}$ Cell Voltage vs. State of Lithiation (a) $10^{\text {th }}$ Cycle (b) $20^{\text {th }}$ Cycle ....... 149

Figure 4-38: Lithiation and Delithiation Capacity and Coulombic Efficiency of LFP/Li Cells

Figure 4-39: (a) Nyquist Plot at $2.5 \mathrm{~V}$ vs. Li/Li ${ }^{+}$After 10 and 20 Cycles (b) Nyquist Plot at $4 \mathrm{~V}$ vs. $\mathrm{Li} / \mathrm{Li}^{+}$After 10 and 20 Cycles for LFP/Li Cells.......................................... 151

Figure 4-40: $\quad$ LFP/Li Cell Voltage vs. State of Lithiation (a) $10^{\text {th }}$ Cycle (b) $20^{\text {th }}$ Cycle ......... 152

Figure 4-41: Assembled Full Cell................................................................................. 154

Figure 4-42: Charge and Discharge Capacity and Coulombic Efficiency of LFP/Cu $\mathrm{Cb}_{2} \mathrm{Cells}$

Figure 4-43. (a) Nyquist Plot at $1.75 \mathrm{~V}$ After 10 and 20 Cycles (b) Nyquist Plot at $3.5 \mathrm{~V}$ After

10 and 20 Cycles for $\mathrm{LFP} / \mathrm{Cu}_{2} \mathrm{Sb}$ Cells ........................................................ 158

Figure 4-44: $\quad \mathrm{LFP} / \mathrm{Cu}_{2} \mathrm{Sb}$ Cell Voltage vs. Depth of Discharge (a) $10^{\text {th }}$ Cycle (b) $20^{\text {th }}$ Cycle 158

Figure 4-45: Charge and Discharge Capacity and Coulombic Efficiency of LFP/LTO Cells 159

Figure 4-46: (a) Nyquist Plot at 1.5 V After 10 and 20 Cycles (b) Nyquist Plot at 2.5 V After 10 and 20 Cycles for LFP/LTO Cells ................................................................. 160

Figure 4-47: LFP/LTO Cell Voltage vs. Depth of Discharge (a) $10^{\text {th }}$ Cycle (b) $20^{\text {th }}$ Cycle.... 161

Figure 5-1: $\quad$ Electrolyte Boiling Facility ....................................................................... 166

Figure 5-2: $\quad$ Electrolyte Boiling Facility: Exploded View of Test Section .......................... 167

Figure 5-3: $\quad$ Interface of PEEK Top and Bottom Flanges with Test Section ....................... 168

Figure 5-4: $\quad$ Steel Top and Bottom Flange Supports .................................................... 168

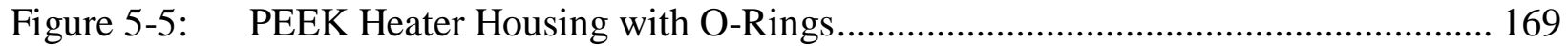

Figure 5-6: $\quad 10 \mathrm{~W}$ Thin Film Heater with Three T-Type Surface Thermocouples ............... 169

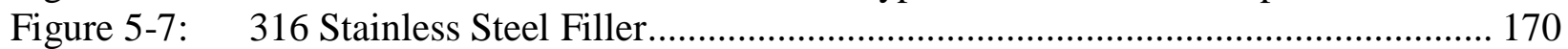

Figure 5-8: $\quad$ Preheater Wire Passage in Stainless Steel Filler .............................................. 170

Figure 5-9: $\quad$ LFP and $\mathrm{Cu}_{2} \mathrm{Sb}$ Electrodes Used in Electrolyte Boiling Facility ...................... 171

Figure 5-10: Electrode Configuration in Electrolyte Boiling Facility ................................ 171

Figure 5-11: Metal Polish and Solvents Used for Cleaning Electrolyte Wetted Components 172

Figure 5-12: $\quad$ Mounting of PEEK Heater Housing onto Stainless Steel Filler with 10 W Thin Film

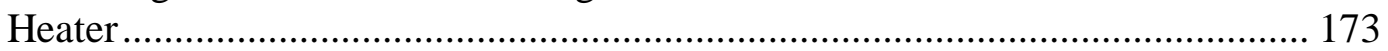

Figure 5-13: Assembled Test Section with Preheater and without Electrodes ...................... 174

Figure 5-14: Assembled Test Section without Preheater and with Electrodes ...................... 174

Figure 5-15: 8-32 Screw with O-Ring Sealing Head...................................................... 175

Figure 5-16: Installed 8-32 O-Ring Screws, Fittings, and PTFE Gasket ............................ 175

Figure 5-17: Assembly of the Test Section with Top Flange and Electrode Leads ................ 175

Figure 5-18: Bottom View of Assembled Test Section with Top Flange and Electrode Leads with

Simulated Sight Glass Bottom Edge ........................................................... 176

Figure 5-19: Final Assembly of Test Section with Sight Glass and Bottom Flange ............... 176 
Figure 5-20: Inserting all Electrolyte Boiling Facility Components into Glove Box via the Large

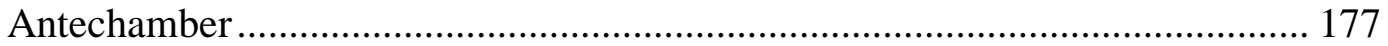

Figure 5-21: Fully Assembled Electrolyte Boiling Facility in Glove Box ............................ 177

Figure 5-22: $\quad$ Modified $180 \mathrm{~mL}$ Glass Jar Lid for Charging the MFE into the Electrolyte Boiling

Facility .................................................................................... 178

Figure 5-23: EBF Charging Process Development with Water........................................... 179

Figure 5-24: EBF System Pressure versus Time with Valve Events.................................. 180

Figure 5-25: Modified Glass Jar Containing MFE Connected to EBF for Charging ............. 180

Figure 5-26: Video Snapshots of MFE Charging Process into the EBF ............................... 181

Figure 5-27: Instek SPS-606 Variable DC Power Supply for 10 W Thin Film Heater.......... 182

Figure 5-28: Thermo Scientific LC 250 Recirculating Chiller, Water Lines, and Glove Box

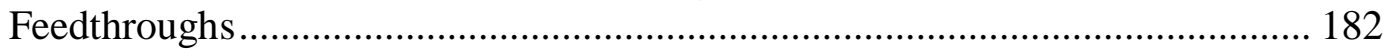

Figure 5-29: National Instruments cDAQ-9174 DAQ Chassis (source: ni.com) .................. 183

Figure 5-30: Wiring of NI 9214 16-Channel Isothermal Thermocouple Input Module......... 183

Figure 5-31: Wiring of NI 9207 Voltage/Current Analog Input Module .............................. 184

Figure 5-32: Glove Box Feedthroughs for NI DAQ and Gamry Potentiostat ....................... 184

Figure 5-33: VI Front Panel for EBF DAQ System ..................................................... 185

Figure 5-34: Pre-Boiling Chronopotentiogram of $\mathrm{LFP} / \mathrm{Cu}_{2} \mathrm{Sb}$ Cell during Cycles 1-10 with System Temperature and Pressure Traces .................................................. 187

Figure 5-35: $\quad \mathrm{LFP} / \mathrm{Cu}_{2} \mathrm{Sb}$ Cell Capacity and Coulombic Efficiency versus Cycle Number..... 188

Figure 5-36: Boiling Chronopotentiogram of LFP/Cu $2 \mathrm{Sb}$ Cell during Cycles 11-20 with System Temperature and Pressure Traces .................................................................. 189

Figure 5-37: View of Evaporation Channel while Cycling LFP/Cu $2 \mathrm{Sb}$ Cell at $0.5 \mathrm{C}$ with $4 \mathrm{~W}$ of Heat Input....................................................................................... 192

Figure 5-38: Bubble Point-Dew Point of HFE-7000/EMC Mixture with Measured Heater and Bulk Electrolyte Temperature from EBF Test ................................................ 194

Figure 5-39: Effect of System Pressure on Bubble Point-Dew Point of HFE-7000/EMC Mixture 195

Figure 5-40: Post-Boiling Chronopotentiogram of $\mathrm{LFP} / \mathrm{Cu}_{2} \mathrm{Sb}$ Cell during Cycles 21-30 with System Temperature and Pressure Traces ..................................................... 196

Figure 5-41: $\quad \mathrm{LFP} / \mathrm{Cu}_{2} \mathrm{Sb}$ Cell Voltage versus Capacity for Cycles 10, 20, and 30 ............... 197

Figure A-1: $\quad 50 \mathrm{~mL}$ Sealed Schlenk Flask Connected to Vacuum Line................................. 215

Figure A-2: $\quad$ Freeze-Pump-Thaw Vacuum Manifold Setup in Fume Hood ........................... 216

Figure A-3: $\quad$ Luke Warm Water Bath for Thawing Frozen Solvents ................................. 217

Figure B-1: $\quad$ Platinum Electrode Polishing on Nylon Disk .............................................. 218

Figure B-2: $\quad$ Electrode Polishing on Microcloth Disk ....................................................... 219

Figure C-1: $\quad$ Surry-Based Electrode Coating Equipment used at Prieto Battery ................... 220

Figure C-2: NMP Solvent, PVDF Binder, and Stainless Steel Ball Bearings in Plastic Jar .. 221

Figure C-3: $\quad$ Paint Can Shaker used for Slurry Homogenizing .......................................... 221

Figure C-4: Weighing Lithium Titanate Oxide Particles Prior to Inserting into Slurry Mixing

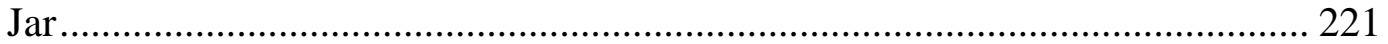

Figure C-5: NMP and Suspended PVDF with Carbon Conductive Additives ..................... 221

Figure C-6: Homogenized LTO Slurry; Ready for Electrode Coating ............................... 222

Figure C-7: $\quad$ Prepared Copper Current Collector with LTO Slurry Globs Prior to First Pass with

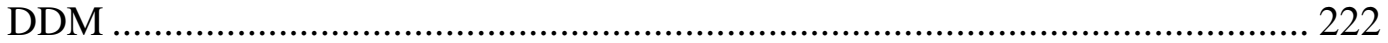

Figure C-8: $\quad$ Coated LTO Electrodes with DDM at Travel Limit ..................................... 223 
Figure C-9: Coated LTO Electrodes under Heat Lamp Prior to Vacuum Oven ................... 223

Figure C-10: Calendaring Electrodes to Final Thickness.................................................. 224 


\section{NOMENCLATURE}

\section{Symbol}

Description

Units

$\alpha \quad$ Exponent for impedance of a constant phase element

a Chemical activity of electrochemically active species

A Area

$\mathrm{m}^{2}$

$B \quad$ Magnitude of magnetic field

$\mathrm{T}$

C Capacity of cell

$\mathrm{Ah}$

$C_{\mathrm{dl}} \quad$ Capacitance of electrochemical double-layer

F

CE Coulombic efficiency

$\%$

CPE Constant phase element, approximates capacitor AC impedance

$\Omega$

C-Rate Rate of charge or discharge normalized by 1 hour (e.g., 1C

discharge requires $1 \mathrm{hr}$ )

$\frac{\partial U}{\partial T} \quad$ Entropic heat coefficient

$\mathrm{V} \mathrm{K}^{-1}$

$\frac{d T}{d x} \quad$ Thermal gradient in $x$ direction

$\mathrm{K} \mathrm{m}^{-1}$

E Energy of cell; Measured electrode potential versus a reference

Wh; V

$E^{0} \quad$ Standard reference potential

V

$f \quad$ Frequency

$\mathrm{Hz}$

F Faraday constant, charge on one mole of electrons

$\mathrm{C}$

HOMO Highest occupied molecular orbital

$\mathrm{eV}$

$i^{\prime \prime \prime} \quad$ Volumetric current generation

$\mathrm{A} \mathrm{m}^{-3}$

I Current, applied or measured

A 


\begin{tabular}{|c|c|c|}
\hline$j$ & Imaginary unit, $\sqrt{-1}$ & - \\
\hline$k$ & Thermal conductivity & $\mathrm{W} \mathrm{m} \mathrm{m}^{-1} \mathrm{~K}^{-1}$ \\
\hline$l$ & Length & $\mathrm{m}$ \\
\hline LUMO & Lowest unoccupied molecular orbital & $\mathrm{eV}$ \\
\hline$m$ & Mass & $\mathrm{kg}$ \\
\hline$\eta$ & Overpotential $\left(U-E^{0}\right)$ & V \\
\hline$\sigma$ & Electronic conductivity & $\mathrm{S} \mathrm{m}^{-1}$ \\
\hline$\phi$ & Potential & $\mathrm{V}$ \\
\hline$q$ & Heat flow & $\mathrm{W}$ \\
\hline$q^{\prime \prime \prime}$ & Volumetric heat generation & $\mathrm{W} \mathrm{m}^{-3}$ \\
\hline$Q$ & Capacitance of constant phase element & $\mathrm{S} \mathrm{s}^{\alpha}$ \\
\hline$R$ & Ideal gas constant, 8.314 & $\mathrm{~J} \mathrm{~mol}^{-1} \mathrm{~K}^{-1}$ \\
\hline$R_{\text {cond }}$ & Conduction thermal resistance & $\mathrm{K} \mathrm{W}^{-1}$ \\
\hline$R_{\mathrm{ct}}$ & Charge-transfer resistance through SEI & $\Omega$ \\
\hline$R_{\Omega}$ & Cell resistance due to conductivity of cell components & $\Omega$ \\
\hline $\mathrm{SOC}$ & State-of-charge & $\%$ \\
\hline SOL & State-of-lithiaton & $\%$ \\
\hline$t$ & Time & $\mathrm{s}$ \\
\hline$T$ & Temperature & K \\
\hline$v$ & Stoichiometric coefficient of redox reaction & - \\
\hline$\mu$ & Chemical potential of electrochemically active species & $\mathrm{kJ} \mathrm{mol}^{-1}$ \\
\hline$\mu_{0}$ & Magnetic permeability in vacuum & $\mathrm{N} \mathrm{A}^{-2}$ \\
\hline
\end{tabular}




\begin{tabular}{|c|c|c|}
\hline Symbol & Description & Units \\
\hline$U$ & Applied potential & $\mathrm{V}$ \\
\hline$V$ & Voltage & $\mathrm{V}$ \\
\hline$w$ & Active material loading & $\mathrm{mg} \mathrm{cm}^{-2}$ \\
\hline$\omega$ & Angular frequency & $\operatorname{rad~s}^{-1}$ \\
\hline$Y$ & Mass fraction & - \\
\hline$Z$ & AC impedance & $\Omega$ \\
\hline
\end{tabular}

\section{Subscripts}

a Activation

AM Active material

aq Aqueous

c Mass concentration

CC Current collector

CE Coated electrode

cell Cell

coat Coating

enc Enclosed

eq Equivalent

g Gas

j Imaginary

$1 \quad$ Liquid

O Oxidized species

$\Omega \quad$ Ohmic 


\begin{tabular}{cl}
\hline $\mathrm{r}$ & Real component \\
$\mathrm{R}$ & Reduced species \\
$\mathrm{s}$ & Solid \\
$\sup$ & Supply \\
$\mathrm{T}$ & Total \\
Theo & Theoretical \\
$\mathrm{x}$ & $x$-direction \\
$0.5 \mathrm{C}$ & $0.5 \mathrm{C}$ rate
\end{tabular}

\section{Abbreviations}

\begin{tabular}{cl}
\hline $\mathrm{AC}$ & Alternating current \\
$\mathrm{B}$ & Baseline electrolyte (1.0 M LiPF 6 in 3:7 EC/DEC by wt) \\
$\mathrm{CE}$ & Coulombic efficiency; counter electrode \\
$\mathrm{CV}$ & Cyclic voltammetry; Charging valve \\
$\mathrm{Cu}_{2} \mathrm{Sb}$ & Copper antimonide, intermetallic negative electrode material \\
$\mathrm{DAQ}$ & Data acquisition \\
$\mathrm{DC}$ & Direct current \\
$\mathrm{DEC}$ & Diethyl carbonate \\
$\mathrm{E} 1$ & Candidate MFE (1.0 M LiTFSI in 1:1 HFE-7000/EMC by vol) \\
$\mathrm{EC}$ & Ethylene carbonate \\
$\mathrm{EIS}$ & Electrochemical impedance spectroscopy \\
$\mathrm{EMC}$ & Ethyl methyl carbonate \\
$\mathrm{EV}$ & Electric vehicle; Evacuation valve
\end{tabular}




\begin{tabular}{cl}
\hline FR & Fire retardant \\
GCE & Glassy carbon electrode \\
HFE & Hydrofluoroether \\
ID & Inside diameter \\
LFP & Lithium iron phosphate, $\mathrm{LiFePO}_{4}$ \\
LIB & Lithium-ion battery(ies) \\
LiPF 6 & Lithium hexafluorophosphate \\
LiTFSI & Lithium bis(trifluromethane sulfonyl) imide, $\mathrm{LiN}\left(\mathrm{SO}_{2} \mathrm{CF}_{3}\right)_{2}$ \\
LTO & Lithium titanate oxide, Li4Ti5 $\mathrm{O}_{12}$ \\
LVL & Lower voltage limit \\
MFE & Multi-functional electrolyte \\
OD & Outside diameter \\
PTE & Platinum electrode \\
RC & Resistor-capacitor circuit, elements connected in parallel \\
RE & Reference electrode \\
SEI & Solid electrolyte interphase \\
SEM & Scanning electron microscope \\
TC & Thermocouple \\
WVL & Thermal management system(s) \\
Upper voltage limit \\
Working electrode \\
\end{tabular}




\section{CHAPTER 1. INTRODUCTION}

Batteries have become an indispensable electrical energy storage medium for countless different consumer, industrial, and defense applications. When Sony produced the first commercial lithium-ion battery (LIB) in 1991, the demand has never ceased to increase. From 2009 to 2015, the demand for the LIB is expected to grow from 4 billion units to over 8 billion units [1]. In this time, LIBs have eclipsed other battery chemistries to become the standard to power most consumer electronics and more recently electric vehicles (EVs) and hybrid electric vehicles (HEVs). LIBs account for nearly three-fourths of the secondary (rechargeable) battery market. A detailed economic analysis projects global LIB demand to grow from $61 \mathrm{GWh}$ in 2015 to $124 \mathrm{GWh}$ by 2020, with $22-41 \%$ compound annual growth for automotive applications during that time [2]. LIBs also have specialized applications in the aerospace industry, aeronautical industry, defense, and grid storage. These applications range from satellite power systems to auxiliary power units for aircraft to solar energy storage for homes.

Despite the growing market and diverse applications, LIBs are still plagued by inherent thermal issues. These thermal issues include capacity fade, self-discharge, and thermal runaway [3]. None of these issues are as catastrophic as thermal runaway: a series of cascading, exothermic reactions that result in fire and possibly explosions. Scientists and engineers have devised numerous thermal management techniques to limit the effects of capacity fade, self-discharge, and thermal runaway. Unfortunately, many of these implemented solutions come at the expense of decreasing volumetric and gravimetric energy density of the pack.

The following sections will provide introductory information for LIBs. First, the motivation for using LIBs will be discussed. Second, the electrochemical basics that guide the function and internal heat generation of a LIB will be presented. Third, the fundamental thermal 
limitations of a LIB will be discussed including thermal runaway and associated failures. Finally, the organization of the remainder of this document is provided.

\subsection{Motivation for Using Li-Ion Batteries}

LIBs are the industry standard for portable energy storage. No other commercially available and rechargeable battery chemistry weighs less and stores as much energy in the same volume. In fact, the next closest competitors to LIBs are nickel metal hydride (NiMH) and nickel cadmium (Ni-Cd) batteries. Compared to these chemistries, LIBs are far superior in specific energy, energy density, and nominal cell voltage. For example, a state-of-the-art LIB has a nominal voltage of $4.2 \mathrm{~V}$, a specific energy of $240 \mathrm{Wh} \mathrm{kg}^{-1}$, and an energy density of $640 \mathrm{Wh} \mathrm{L}^{-1}$. In contrast, a NiMH battery has a nominal voltage of $1.2 \mathrm{~V}$, a specific energy of $75 \mathrm{Wh} \mathrm{kg}^{-1}$, and an energy density of $240 \mathrm{Wh} \mathrm{L}^{-1}$. In addition, a Ni-Cd battery has a nominal voltage of $1.2 \mathrm{~V}$, a specific energy of $35 \mathrm{Wh} \mathrm{kg}^{-1}$, and an energy density of $100 \mathrm{Wh} \mathrm{L}^{-1}$. In other words, over three $\mathrm{NiMH}$ or Ni-Cd cells must be placed in series to produce the same voltage as a single LIB; NiMH require $2.67 \times$ and $\mathrm{Ni}-\mathrm{Cd}$ require $6.4 \times$ the amount of space to have the equivalent stored energy of a LIB; and NiMH weigh $3.2 \times$ and $\mathrm{Ni}-\mathrm{Cd}$ weigh $6.9 \times$ more than a LIB. To further illustrate the advantages of LIBs, Figure 1-1 shows the size and mass of a 640 Wh battery in each of the aforementioned battery chemistries.

Secondary LIBs are also highly efficient. All batteries operate through the use of a pair of oxidation-reduction (redox) reactions whose

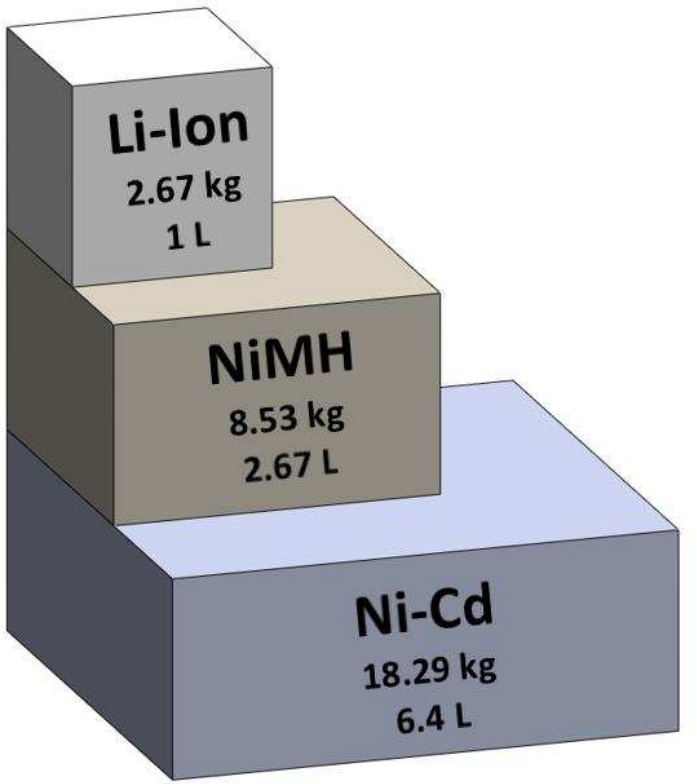
difference in standard reduction potentials over

Figure 1-1: LIBs Compared to NiMH and Ni-Cd (Size and Weight Values are for 640 Wh battery) 
their state of charge determines the voltage of the battery. Oxidation reactions are classified by the electrochemically active species losing an electron to increase its formal charge. The opposite reaction is termed reduction, where the electrochemically active species gains an electron to decrease its formal charge. The transfer of electrons associated with the change of formal charge of the electrochemically active species is what creates the useable energy in a battery. In the case of LIBs the redox reactions can be understood by the change in the formal charge of lithium, the electrochemically active species:

$$
L i \underset{\text { Reduction }}{\stackrel{\text { Oxidation }}{\rightleftharpoons}} \mathrm{Li}^{+}+e^{-}
$$

To enable their high efficiencies, redox reactions in rechargeable battery chemistries are highly reversible. For properly functioning rechargeable LIBs, the ratio of charge capacity output to capacity input is commonly $>99 \%$. If the efficiency of the redox reactions in a LIB is much less than $99 \%$, then it is not a viable chemistry for a rechargeable battery. The efficiency coupled with the high nominal cell voltage, specific energy, and energy density is why LIBs are used frequently in electric vehicle (EV) and hybrid-electric vehicle (HEV) power systems. By comparison, the efficiency of internal combustion (IC) engines can be as low as $25 \%$, with the remaining chemical potential of the fuel lost as heat to the environment [4]. Waste heat recovery efforts can be employed in an attempt to recapture some of the lost heat energy; however, the additional component cost and minimal efficiency gains often limit their application. Battery-powered vehicles also have the benefit of capturing energy that would normally be lost during braking. When the driver presses the brake, the electric motor's polarity is flipped which turns the motor into a generator that charges the battery. This converts the kinetic energy of the car to electricity that it is normally lost to frictional heat with conventional braking systems [5]. This further increases the already high energy efficiency of a HEV and EV. 
Despite the improved efficiency over IC engines, EVs still face challenges that limit their widespread commercial adoption. These challenges include high battery cost (approximately $\$ 400$ $\left.\mathrm{kWh}^{-1}[6]\right)$, battery life uncertainty, and restricted driving range. The stored energy volumetric and gravimetric density of a state-of-the-art LIB pales in comparison to gasoline, which is approximately 9,400 $\mathrm{Wh} \mathrm{L}^{-1}$ and $13,000 \mathrm{Wh} \mathrm{kg}^{-1}$ respectively [7]. The order of magnitude difference in energy density is the primary impediment to designing an EV with comparable driving range to a gasoline-powered vehicle. This forces the LIB packs in EVs to be much larger and heavier in comparison to a gasoline tank. The highest stored energy in a LIB pack for an EV is found in the $90 \mathrm{kWh}$ Tesla Model S which translates to 270 miles of driving range; however, nearly 29\% percent of the Model S vehicle weight is the LIB pack according to one estimate [8]. This will continue to be a significant tradeoff for EVs.

Another significant market concern for EVs is fast charging. Consumers looking to make the switch from fossil fuel-powered vehicles are accustomed to filling a gasoline tank in a few minutes; charging a LIB pack takes much longer. Charge time depends upon the charging infrastructure: AC Level 1 (120 V, 12 A), AC Level 2 (240 V, 40-80 A), and DC fast charging. The longer charging times from AC Level 1 and 2 chargers are supplemented by its convenience; these charging infrastructures can be installed in the home of the consumer. DC fast charging requires a much more robust charging infrastructure for the higher charging currents but can dramatically reduce charging times. Tesla has created a Supercharger network that supply $120 \mathrm{~kW}$ of DC power to rapidly charge its LIB packs. On its website, Tesla advertises that its Superchargers are capable of adding up to 170 miles of range in 30 minutes to a LIB pack when starting with 10\% charge remaining. (Charging a Tesla $85 \mathrm{kWh}$ LIB pack with an AC Level 1 charger would require 61 hours to obtain a full charge.) These DC fast charging stations will continue to evolve 
and more EVs will be designed to charge on this infrastructure; the current SAE standard being developed calls for target charging power levels up to $130 \mathrm{~kW}[6]$.

LIBs also have high compatibility with renewable energy sources. As the world strives to reduce its fossil fuel consumption, the use of renewable sources of energy continues to grow, particularly wind and solar [9]. The growth in renewable energy consumption over the last three years as well as the projected growth in the United States is shown in Figure 1-2. Solar and wind energy consumption are projected to increase by $20 \%$ and $16 \%$ respectively in 2016. However, solar and wind energy consumption accounted for only a very small fraction $(2.6 \%)$ of the total energy usage in the United States in 2015 [10]. To more effectively utilize renewable energy generated from solar and wind, especially

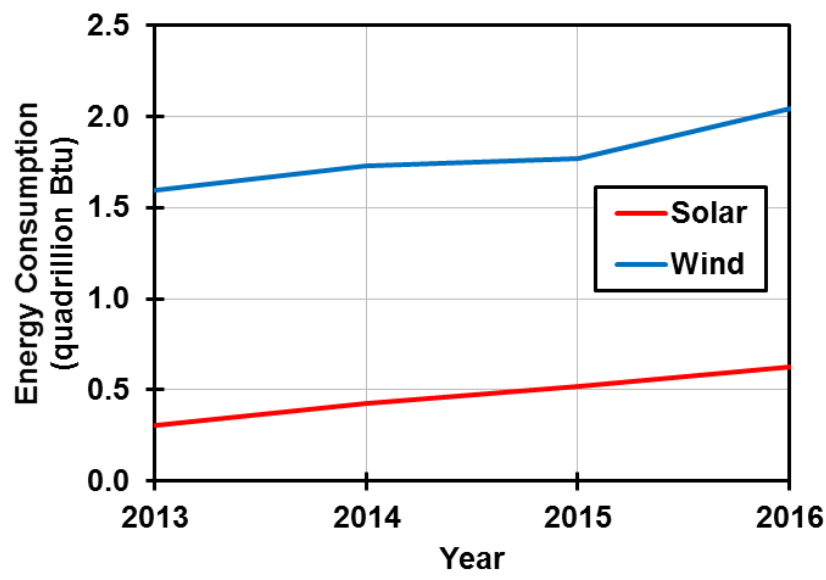

Figure 1-2: US Solar and Wind Energy Consumption [9]

in times of minimum grid demand, an electrical energy storage medium can be used. Solutions for grid storage require daily usage and appreciable lifetimes ( $>10$ years) to be cost-effective. LIBs are a potential solution for grid energy storage, particularly with home solar and wind energy systems. These alternative energy systems output DC power which can be directly input into a battery without the cost of a DC-AC inverter. Tesla has recently released its modular solution for grid energy storage called Powerwall, a \$3,000 6.4 kWh LIB pack. Homeowners can install the Powerwall and program it to store energy from roof-mounted solar panels or to charge off of the grid at low energy demand times (e.g., when the energy cost is low). 


\subsection{Function of a Secondary Li-Ion Battery}

Every LIB contains three basic components: an anode, cathode, and electrolyte. Each of these components can take a variety of different forms. Today's anodes and cathodes are lithium insertion electrodes: the active materials are capable of reversible insertion and extraction (also called intercalation and de-intercalation) of lithium ions. The active materials are also stable enough to store the lithium for long periods of time which enables the primary function of a rechargeable battery: store electrical energy until it is required. Anodes and cathodes have different redox potentials for lithium ion intercalation and de-intercalation reactions. The difference between the redox potentials of the anode and cathode over the entire range of charge is what determines the voltage of the battery. The electrolyte supplies the oxidized and reduced species for LIB operation: lithium ions. The electrolyte must maintain sufficient wetting of the anode and cathode to supply lithium ions to electrochemically active surfaces of the active materials, contain a high concentration of lithium ions $(\sim 1 \mathrm{M})$, and be electrically insulating so the anode and cathode do not short. A brief introduction into the most common chemistries for the anode, cathode, and electrolyte are presented in the following sections.

\subsubsection{Anodes}

Lithium metal is the ideal anode for LIBs. It has an extremely high specific capacity of $3860 \mathrm{mAh} \mathrm{g}^{-1}$, low density of $0.59 \mathrm{~g} \mathrm{~cm}^{-3}$, and contains the most electronegative potential of any electrochemical couple. Lithium metal anodes yield LIBs that are lighter and more power dense than every other candidate LIB anode material. However, the implementation of lithium metal as an anode in LIBs is hindered by the growth of dendrites, which preferentially form when charging the battery at higher rates. These dendrites are capable of piercing the separator between the anode and cathode, shorting the battery and instantaneously releasing the stored energy of cell causing 

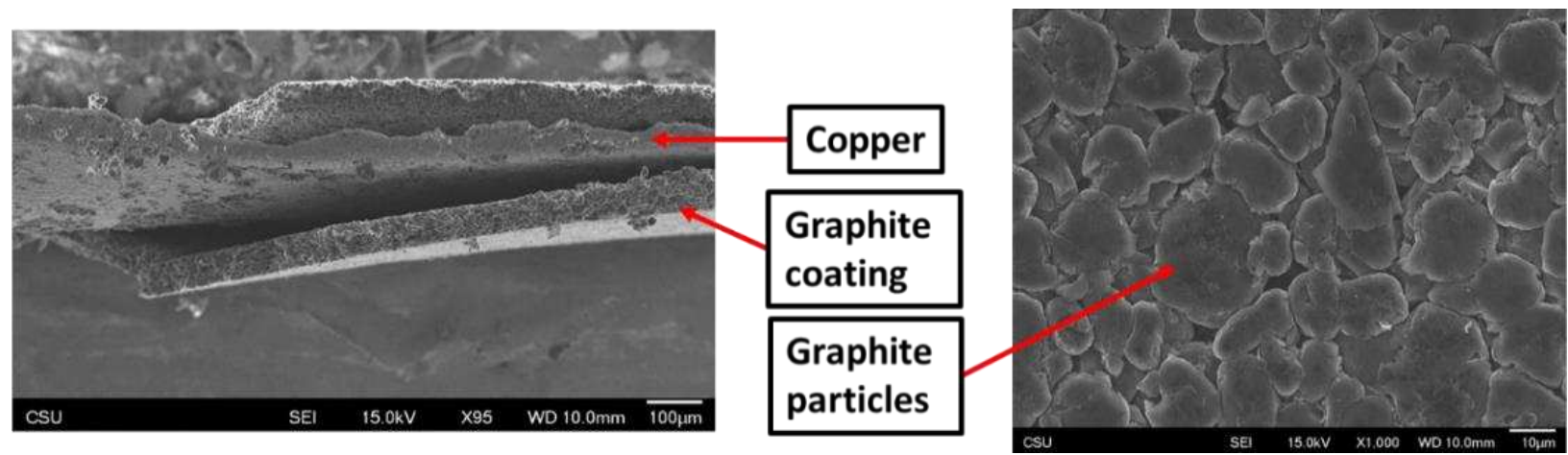

Figure 1-3: SEM Images of Graphite Anodes

thermal runaway (Section 1.3.1). In addition, the lithium plating and stripping process associated with its electrochemical couple is not fully-reversible. The lithium metal plates into a porous structure that requires additional passivation by the electrolyte, even if the battery is charged at a very low rate. This causes continual performance degradation consistent with SEI decomposition and reformation on the porous structure. Further discussion on lithium metal anodes for rechargeable LIBs can be found in the review written by $\mathrm{Xu}$ et al. [11].

Due to the issues with lithium metal, LIB anodes are primarily manufactured from graphite particles coated on a thin sheet of copper. The graphite particles are typically $15 \mu \mathrm{m}$ in diameter and are coated $60 \mu \mathrm{m}$ thick onto a $10 \mu \mathrm{m}$ thick copper sheet (Figure 1-3). The redox reaction of lithium intercalation or de-intercalation into graphite is:

$$
C+y L i^{+}+y e^{-} \underset{\text { Discharge }}{\stackrel{\text { Charge }}{\rightleftharpoons}} L i_{y} C
$$

Potentials at which LIB redox reactions occur are often referenced versus the $\mathrm{Li} / \mathrm{Li}^{+}$redox reaction:

$$
L i^{+}+e^{-} \rightleftharpoons L i_{(\mathrm{s})}^{0}
$$

Note that the $\mathrm{Li}^{0}$ state is lithium in its solid state, meaning that lithium has been plated on the electrode surface if lithium ions have been reduced. This is an important consideration in the selection of anode active materials. Reversible anode active materials in LIBs will always have a redox potential that is greater than the $\mathrm{Li} / \mathrm{Li}^{+}$redox couple to avoid the lithium plating reaction 
(and subsequent likelihood of dendrite formation). Standard reduction potentials found in chemistry textbooks are all typically in reference to the standard hydrogen electrode or normal hydrogen electrode (SHE or NHE) which is governed by the redox reaction:

$$
2 H^{+}+2 e^{-} \rightleftharpoons H_{2(\mathrm{~g})}
$$

The absolute electrode potential for the SHE reaction occurs at $0.41 \mathrm{~V}$. This voltage is considered the zeroing point for all standard reduction potentials. The $\mathrm{Li} / \mathrm{Li}^{+}$redox reaction occurs at $-3.04 \mathrm{~V}$ vs. SHE; the $\mathrm{Li} / \mathrm{Li}^{+}$redox potential versus itself is $0 \mathrm{~V}$. For graphite, the redox potential varies depending upon the lithiation state of the graphite particle (i.e. how much lithium is stored in the graphite structure). The theoretical maximum lithium storage capacity in graphite is $\mathrm{LiC}_{6}$, or one lithium

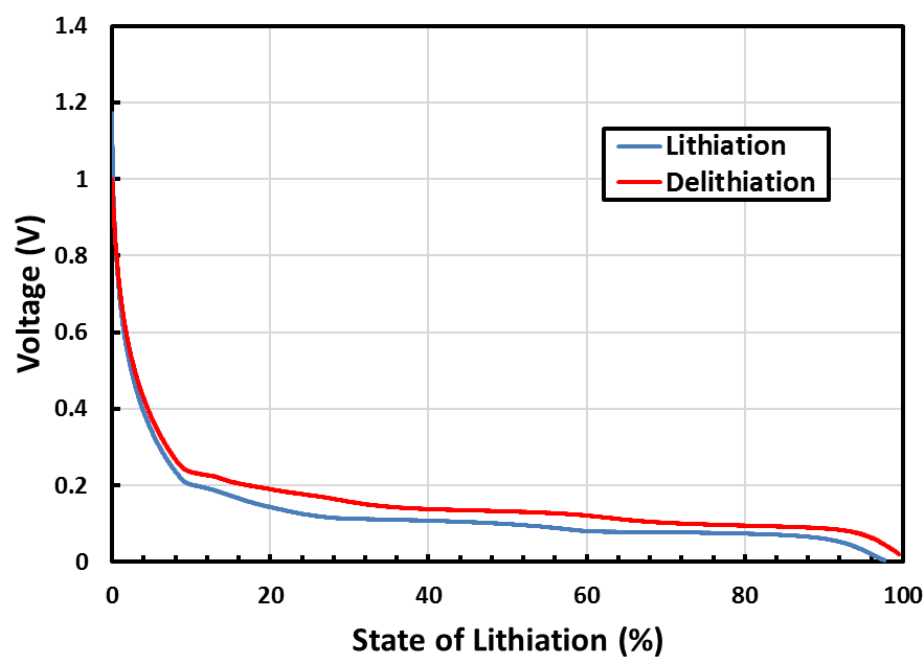

Figure 1-4: Lithiation and Delithation of Graphite at C/30 Rate, Voltage vs. $\mathrm{Li} / \mathrm{Li}^{+}$

equivalent to every six carbon. If the graphite particle is fully lithiated (charged state), the redox potential is typically around $0.05 \mathrm{~V}$ vs. $\mathrm{Li} / \mathrm{Li}^{+}$; in a fully delithiated state, the redox potential is typically around $0.8 \mathrm{~V}$ vs. $\mathrm{Li} / \mathrm{Li}^{+}$. This can be visualized in Figure 1-4.

The copper sheet is called the anode current collector; it is the source and sink for electrons for the redox reactions of lithium intercalation or de-intercalation in graphite. Copper is chosen because of its very high electric conductivity and relatively low cost compared to other high electric conductivity materials (silver and gold). Graphite is commonly found in LIBs because it is capable of reversibly cycling and has a very low potential versus $\mathrm{Li} / \mathrm{Li}^{+}$which enables cells with 
higher nominal voltages. However, the reversibility of graphite as an active material is somewhat limited by the subsequent volume expansion (up to 10\% [12]) in its fully lithiated state from its original delithiated state. Fatigue from the mechanical expansion and contraction of the graphite structure during charge and discharge is one of the primary capacity fade mechanisms currently being investigated in commercial LIBs [13].

There are many other anode chemistries that are functional for LIBs, all of which have higher redox potentials versus $\mathrm{Li} / \mathrm{Li}^{+}$than graphite. This can cause the nominal cell voltage to significantly reduce, which reduces the benefit of the li-ion chemistry over the Ni-Cd and NiMH chemistries. For example, lithium titanate oxide $\left(\mathrm{LTO}, \mathrm{Li}_{4} \mathrm{Ti}_{5} \mathrm{O}_{12}\right)$ is a highly reversible anode active material. However, LTO has a redox potential versus $\mathrm{Li} / \mathrm{Li}^{+}$of $1.5 \mathrm{~V}$. If LTO is selected over graphite as the anode active material, the nominal cell voltage of the LIB is reduced by over $1 \mathrm{~V}$. This causes a significant drop in the stored energy of the cell. However, LTO is considered a no-strain active material: its lattice expansion and contraction upon lithiation and delithiation is less than $1 \%$ [14]. This property alone eliminates the primary failure mechanism that plagues graphite anodes and enables high capacity retention and long cycle life.

An entirely different class of anodes for LIBs are intermetallics. Intermetallic anodes produce useful capacity by the formation of intermetallic phases with lithium and a base metal, $M$. The base metal can be $\mathrm{Mg}, \mathrm{Si}, \mathrm{Sn}, \mathrm{Sb}$, among many others [15]. The general reaction for an intermetallic anode is:

$$
L i_{x} M \underset{\text { charge }}{\stackrel{\text { discharge }}{\rightleftharpoons}} x L i^{+}+x e^{-}+M
$$

These intermetallic anodes have very high theoretical specific charge capacities. For example, a silicon anode has a theoretical charge capacity of $4200 \mathrm{mAh} \mathrm{g}^{-1}$ when alloyed to form $\mathrm{Li}_{15} \mathrm{Si}_{4}$ over 11 times that of graphite [16]. However, the subsequent volume expansion for the Si metallic 
structure to accommodate the lithium is approximately $370 \%$ [16]. This substantial expansion is the primary drawback of using intermetallic anodes over graphite. The volumetric expansion of intermetallic anodes can be reduced by the addition of a third, non-reacting metal, at the expense of the high specific capacity. One such example is copper antimonide $\left(\mathrm{Cu}_{2} \mathrm{Sb}\right)$. In this instance, non-reacting copper is alloyed with the reacting metal, antimony, to create the base metal structure. $\mathrm{Cu}_{2} \mathrm{Sb}$ has a theoretical specific capacity similar to that of graphite, $323 \mathrm{mAh} \mathrm{g}^{-1}$, and a volumetric expansion of only $42 \%$ when fully reacted to form $\mathrm{Li}_{3} \mathrm{Sb}$ [17]. Further discussion is provided on $\mathrm{Cu}_{2} \mathrm{Sb}$ in Section 4.4.1.

\subsubsection{Cathodes}

LIB cathodes are much more diverse and vary significantly depending upon power and energy requirements. A typical high energy LIB cathode will contain crystalline active material particles $(\sim 15 \mu \mathrm{m}$ in diameter $)$ coated $60 \mu \mathrm{m}$ thick onto a thin, $15 \mu \mathrm{m}$ sheet of aluminum. High power LIBs will have cathode active material particles that are $<1 \mu \mathrm{m}$ in diameter to reduce the diffusion lengths of li-ions into the active material particle. The variation in the size of active material particles can be seen in Figure 1-5. The size of the active material particle dramatically affects the rate capability of battery: smaller diameter active material particles have a greater surface area to volume ratio and smaller diffusion lengths. Redox reactions occur only at

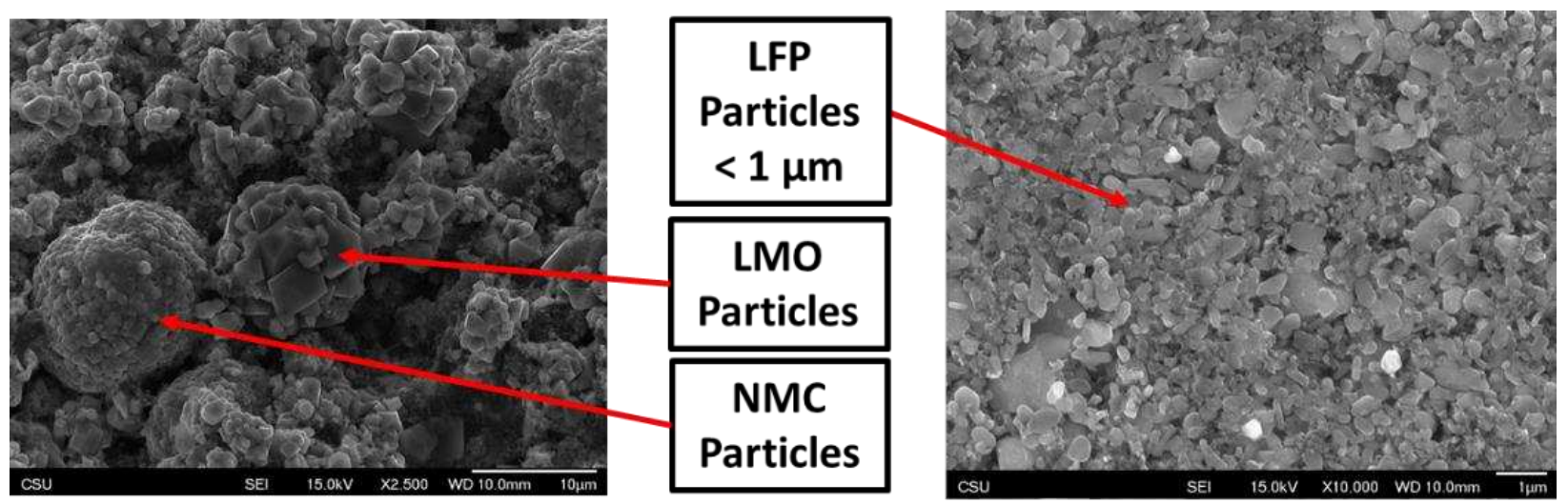

Figure 1-5: SEM Images of Cathode Active Material Particles 
interfaces; therefore, if the active material particle has a large surface area, it will have more electrochemically active area in contact with the electrolyte and a greater rate capability.

Most of the cathode active materials consist of metal oxides or metal phosphates. The most common formulations include lithium manganese oxide ( $\left.\mathrm{LMO}, \mathrm{LiMn}_{2} \mathrm{O}_{4}\right)$, lithium cobalt oxide $\left(\mathrm{LCO}, \mathrm{LiCoO}_{2}\right)$, lithium nickel manganese cobalt oxide (NMC, $\mathrm{LiNi}_{1 / 3} \mathrm{Mn}_{1 / 3} \mathrm{Co}_{1 / 3} \mathrm{O}_{2}$ or $\mathrm{LiNi}_{1 / 2} \mathrm{Mn}_{1 / 3} \mathrm{Co}_{1 / 6} \mathrm{O}_{2}$ ), lithium nickel cobalt aluminum oxide (NCA, $\mathrm{LiNi}_{0.8} \mathrm{Co}_{0.15} \mathrm{Al}_{0.05} \mathrm{O}_{2}$ ), and lithium iron phosphate ( $\left.\mathrm{LFP}, \mathrm{LiFePO}_{4}\right)$. The redox reaction for lithiation and delithiation of LMO is as follows:

$$
L i M n O_{2} \underset{\text { Discharge }}{\stackrel{\text { Charge }}{\rightleftharpoons}} L_{1-x} \mathrm{MnO}_{2}+x \mathrm{Li}^{+}+x e^{-}
$$

This redox reaction is similar for all the other cathode active materials. There are countless other derivatives of these metal oxides and phosphates that have been investigated and patented that seek to increase the redox potential versus $\mathrm{Li}^{\prime} \mathrm{Li}^{+}$to produce a higher voltage and energy battery. Some of the new cathode active materials have redox potentials up to $5.5 \mathrm{~V}$ vs. $\mathrm{Li}^{2} / \mathrm{Li}^{+}$[18]. Regardless of the chemistry, each of these active materials have a different redox potential versus $\mathrm{Li} / \mathrm{Li}^{+}$, and the redox potential changes as a function of lithium content. The redox potentials versus $\mathrm{Li} / \mathrm{Li}^{+}$for NMC, LFP, and a blend of LMO and NMC are shown in Figure 1-6. The difference between the potentials is due to the difference in the work functions of cathode active materials, which is the amount of energy required for electron transfer for lithium oxidation or reduction [19]. The LMO/NMC blended cathode will deliver the highest nominal cell voltage and stored energy density, whereas the LFP cell will have the lowest nominal cell voltage and similarly low stored energy density. 
The selection of the cathode active material in a LIB is primarily driven by application, manufacturing cost, and safety. In instances of high power (drawing large currents from the battery), LFP is the best option as it is capable of high rate performance with minimal material degradation.

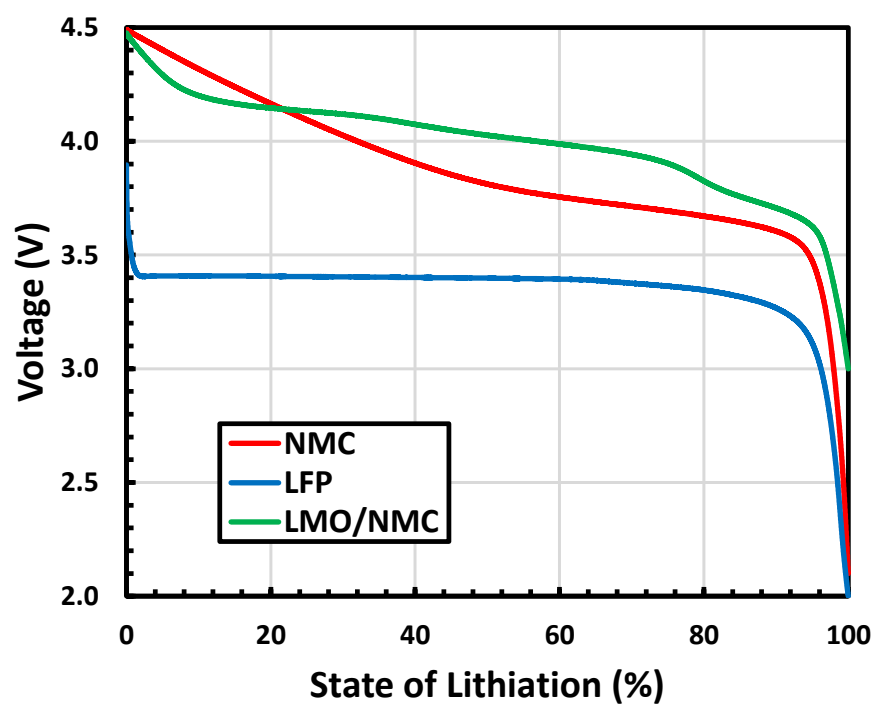
However, in applications that Figure 1-6: Lithiation of Cathode Active Materials at C/30 Rate, Voltage vs. $\mathrm{Li} / \mathrm{Li}^{+}$

demand high energy storage, LFP is not the best option because its volumetric capacity is smaller than for other chemistries and it has the lowest redox potential versus $\mathrm{Li} / \mathrm{Li}^{+}$of common cathode active materials. Table 1-1 contains properties of some commonly applied cathode active materials. It is common to find various derivatives of cathode active material particles. In Table 1-1, NMC has two different formulations that yield similar results for gravimetric and volumetric capacity. NMC 2 contains less cobalt, and is consequently less expensive and more thermally stable than NMC 1; however, NMC 2 has a lower rate capability [20]. Managing these tradeoffs encompasses the active material selection process for designing LIBs which is well-illustrated in the technical paper written by Matthe et al. on the Chevrolet Volt [21].

Table 1-1: Physical Properties of Some Commonly Applied Cathode Active Materials

\begin{tabular}{llcccc} 
Material & Chemical Formula & $\begin{array}{c}\text { Crystallographic } \\
\text { Density } \\
\mathrm{g} \mathrm{cm}^{-3}\end{array}$ & $\begin{array}{c}\text { Gravimetric } \\
\text { Capacity } \\
\mathrm{mAh} \mathrm{g}^{-1}\end{array}$ & $\begin{array}{c}\text { Volumetric } \\
\text { Capacity } \\
\mathrm{mAh} \mathrm{cm}^{-3}\end{array}$ & $\begin{array}{c}\mathbf{E}^{\mathbf{0}} \mathbf{\text { vs. }} \\
\mathbf{L i}^{-\mathbf{L i}^{+}} \\
\mathrm{V}\end{array}$ \\
\hline $\mathrm{LMO}$ & $\mathrm{LiMn}_{2} \mathrm{O}_{4}$ & 4.37 & 148 & 648 & $3.8-4.2$ \\
$\mathrm{NMC} 1$ & $\mathrm{Li}\left(\mathrm{Ni}_{1 / 3} \mathrm{Mn}_{1 / 3} \mathrm{Co}_{1 / 3}\right) \mathrm{O}_{2}$ & 4.77 & 280 & 1334 & $3-4.5$ \\
$\mathrm{NMC} 2$ & $\mathrm{Li}_{\left(\mathrm{Ni}_{1 / 2} \mathrm{Mn}_{1 / 3} \mathrm{Co}_{1 / 6}\right) \mathrm{O}_{2}}$ & 4.77 & 278 & 1329 & $3-4.5$ \\
$\mathrm{LFP}$ & $\mathrm{LiFePO}_{4}$ & 3.60 & 170 & 612 & $3-3.5$
\end{tabular}




\subsubsection{Electrolytes}

LIB electrolytes can take several different forms and phases: liquid, polymer, solid, or ionic liquid. In general, liquid electrolytes have low viscosity, high ionic conductivity, and are extremely flammable. Polymer electrolytes have a significantly higher viscosity and lower ionic conductivity, but still contain the similar components that make liquid electrolytes extremely flammable. Certain ceramic structures are capable of li-ion conduction and can serve as an electrolyte; however, the temperature at which the lithium diffusion processes become functional is much higher than the practical use temperatures of a LIB. Ionic liquids (molten salts) have the highest conductivity, but must be maintained at a high temperature to remain in the liquid state to be an ionically-conducting electrolyte.

The state-of-the-art liquid electrolytes for LIBs are a mixture of two to three nonaqueous organic carbonate solvents with an inorganic lithium salt. Common organic carbonate solvents include ethylene carbonate (EC), dimethyl carbonate (DMC), ethyl methyl carbonate (EMC), and diethyl carbonate (DEC). Of these organic carbonate solvents, DMC, EMC, and DEC are extremely flammable, all having very low flash points. EC is found in almost every liquid electrolyte due to its high dielectric constant, which increases its capability to solvate inorganic lithium salts, and its ability to form and maintain interfacial stability on both the anode and cathode [22]. EC cannot serve as the sole solvent for LIB electrolytes because it is a solid at room temperature. However, EC readily mixes with DMC, EMC, and DEC to form electrolyte solutions that have low viscosities (mixtures can remain liquids at temperatures down to $-30^{\circ} \mathrm{C}$ ) that enable li-ion transport with ionic conductivities ranging from $5-10 \mathrm{mS} \mathrm{cm}^{-1}$ [23].

LIBs do not contain lithium metal. The source of lithium ions is lithium salt that is solvated to form the electrolyte. Lithium salts are categorized as inorganic or organic; organic salts contain 
carbon, whereas inorganic do not. The primary difference is evident in the chemical structure and the size of the anion when the salt is solvated. Inorganic salts have much smaller anions, and consequently require solvents that have higher dielectric constants in order to be solvated and remain solvated. The larger anions of the organic salts have a much greater charge distribution and can be solvated with fluids that have much lower dielectric constants. In addition, large anion size will typically result in a higher electrolyte viscosity, which causes lower ionic conductivity [24].

The most common inorganic lithium salt is lithium hexafluorophosphate $\left(\mathrm{LiPF}_{6}\right) . \mathrm{LiPF}_{6}$ has been the LIB industry standard for a lithium salt due to its high ionic conductivity and stability with a variety of cathode and anode chemistries after initial SEI formation cycles have been completed. Unfortunately, $\mathrm{LiPF}_{6}$ has very poor thermal stability and can readily form hydrofluoric acid when exposed to water. Other inorganic lithium salts include lithium perchlorate $\left(\mathrm{LiClO}_{4}\right)$ and lithium tetrafluoroborate $\left(\mathrm{LiBF}_{4}\right)$, but are not common in commercial products due to their poor compatibility with the cathode aluminum current collector at high potentials. Organic lithium salts have been extensively investigated, but are not typically found in commercial LIBs due to their lower ionic conductivities than inorganic salts. The most prominent include lithium bis(trifluoromethane)sulfonimide $\quad$ (LiTFSI, $\left.\quad \mathrm{LiN}\left(\mathrm{SO}_{2} \mathrm{CF}_{3}\right)_{2}\right) \quad$ and $\quad$ lithium bis(perfluoroethylsulfonyl)imide ( $\left.\mathrm{LiBETI}, \mathrm{LiN}\left(\mathrm{SO}_{2} \mathrm{C}_{2} \mathrm{~F}_{5}\right)_{2}\right)$. The attraction of using organic salts originates from their high solubility in solvents that have much lower dielectric constants and high thermal stability. Their high solubility is due to the negative charge distribution that spreads over the large anion molecule of the organic salt (the $\left[\mathrm{N}\left(\mathrm{SO}_{2} \mathrm{CF}_{3}\right)_{2}\right]^{-1}$ anion of LiTFSI salt is much larger than the $\left[\mathrm{PF}_{6}\right]^{-1}$ anion of $\mathrm{LiPF}_{6}$ salt). Therefore, once an organic salt is solvated, it is highly unlikely that the lithium ion will be attracted to its bonding site on the anion because there is a minimal 
charge attraction. Their high thermal stability is due to the plethora of $\mathrm{C}-\mathrm{F}$ bonds in the anion, which are far stronger bonds than the P-F bonds of $\mathrm{LiPF}_{6}$ [25].

\subsubsection{Separators}

Separators for LIBs must be electrically insulating, allow for lithium-ion migration, and provide enough mechanical strength to withstand cell manufacturing processes. Microporous polyolefins such as polypropylene (PP) and polyethylene (PE) are commonly used as LIB separator materials. Either of these materials can be manufactured

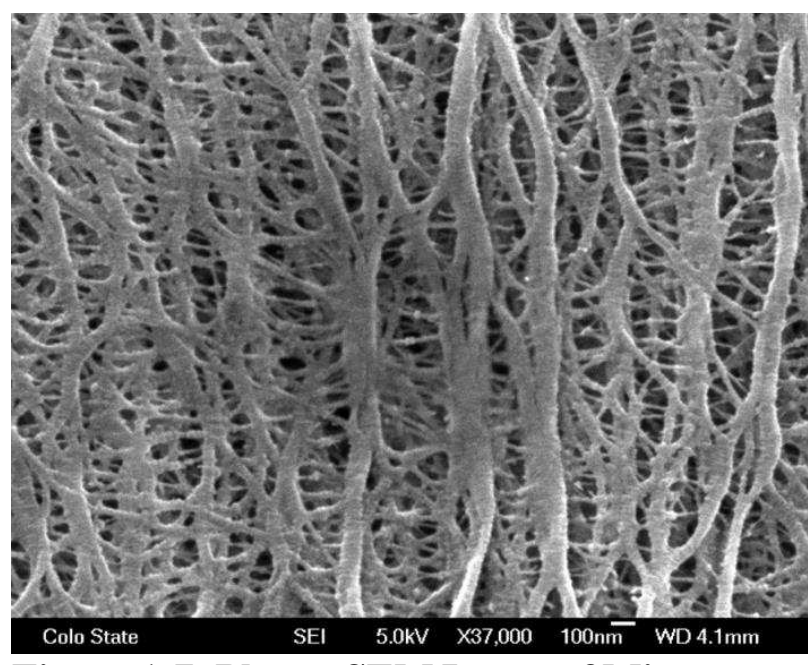

Figure 1-7: Planar SEM Image of Microporous PP Separator into a thin $\sim 20-25 \mu \mathrm{m}$ sheet with $>40 \%$ porosity with a pore size ranging from $0.03-0.1 \mu \mathrm{m}[26$, 27]. A SEM image of a PP separator is shown in Figure 1-7. The PP is clearly elongated in a preferential direction and has significant porosity. A common commercially-produced LIB separator manufactured by Celgard is a trilayer structure of PP/PE/PP which has a combined thickness of $25 \mu \mathrm{m}$. This architecture affords inherent thermal protection in the event of excessively high battery temperatures. The PE structure melts at $135^{\circ} \mathrm{C}$ (below that of $\mathrm{PP}, 165^{\circ} \mathrm{C}$ ) behaving as a thermal fuse to any further lithium-ion transfer [26]. Separator materials are not limited only to polyolefins. As mentioned in Section 1.2.3, the development of solid electrolytes precludes the need for a conventional separator. One such example is the glass-ceramic $\mathrm{Li}_{2} \mathrm{~S}-\mathrm{P}_{2} \mathrm{~S}_{5}$ which is capable of lithium-ion conduction and electrical insulation. However, solid electrolytes have yet to see commercial adoption due to inferior performance compared to liquid electrolytes [28]. 


\subsubsection{Li-Ion Battery Function}

Charging and discharging a

LIB causes equal, but opposite redox reactions at the anode and cathode. In all instances of LIB operation, charge neutrality at the anode and cathode is maintained. For illustrative purposes, the anode active material is graphite and the cathode active material is LMO in

Figure 1-8. During charge, lithium

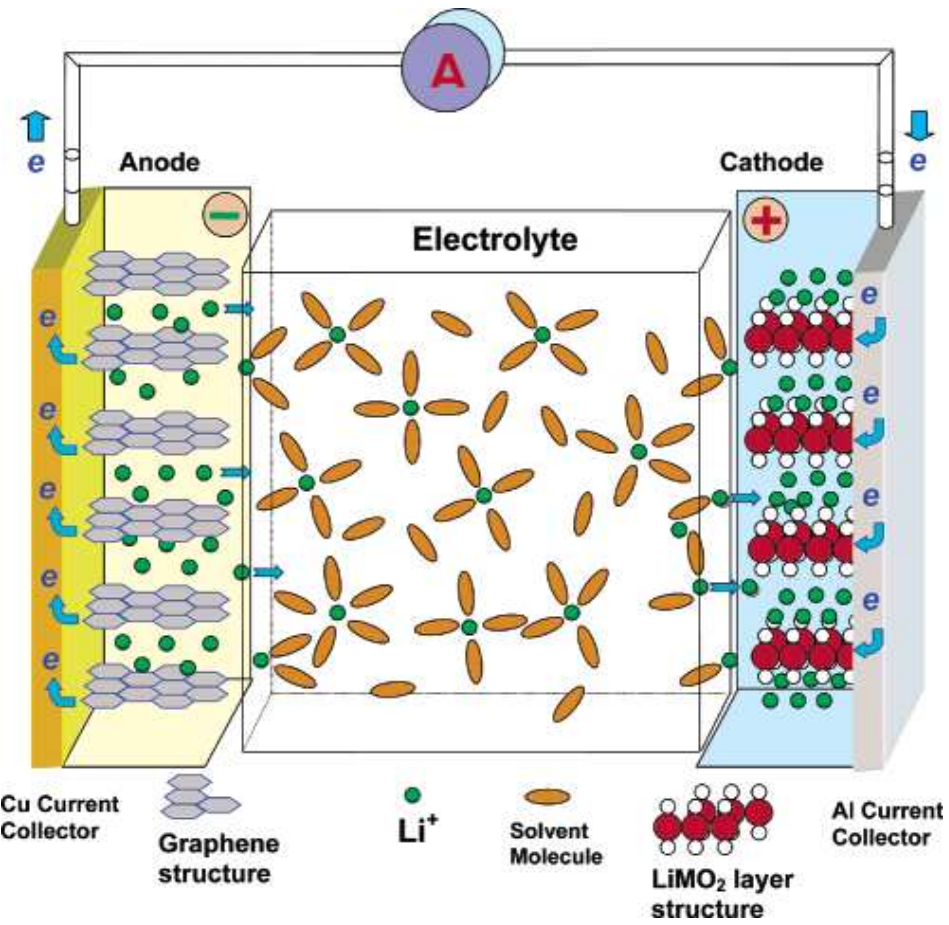

Figure 1-8: Schematic of a LIB During Discharge [24] ions intercalate into the graphite active material in the anode from the electrolyte. The intercalation into graphite causes the potential of the anode versus $\mathrm{Li} / \mathrm{Li}^{+}$to decrease. In its fully lithiated state, the potential of graphite is $0.05 \mathrm{~V}$ vs. $\mathrm{Li} / \mathrm{Li}^{+}$. Simultaneously, lithium ions de-intercalate from the LMO active material into the electrolyte at the same rate as the anode. The de-intercalation of LMO causes the cathode potential to rise versus $\mathrm{Li} / \mathrm{Li}^{+}$, reaching a maximum of $4.5 \mathrm{~V}$ in the fully delithiated condition. The electrons required for the reduction of lithium into graphite during the intercalation process is supplied from the oxidation of lithium from LMO. The electrons travel from the aluminum current collector, through the external circuit attached to the battery, and ultimately to the copper current collector. The total cell voltage in the fully-charged state is simply determined as $4.5-0.05 \mathrm{~V}=4.45 \mathrm{~V}$.

During discharge (Figure 1-8), the graphite delithiates, oxidizing its stored lithium back into the electrolyte. This causes the voltage of the anode to increase, eventually reaching $0.8 \mathrm{~V}$ vs. 
$\mathrm{Li} / \mathrm{Li}^{+}$. Subsequently, the LMO lithiates, reducing lithium into its structure from the electrolyte. The reduction of lithium causes the voltage of the cathode to decrease, eventually reaching $3 \mathrm{~V}$ vs. $\mathrm{Li} / \mathrm{Li}^{+}$. The electrons travel from the copper current collector, through the external circuit attached to the battery, and ultimately to the aluminum current collector. The total cell voltage in the discharged state is determined as $3-0.8 \mathrm{~V}=2.2 \mathrm{~V}$.

The charge and discharge rate of a LIB is defined in terms of a C-Rate. The C-Rate is determined from the charge capacity of the battery $\left(C_{\text {cell }}\right)$ and is normalized by the current, $I$, required to fully discharge the battery in 1 hour (i.e., a $1 \mathrm{C}$ discharge current will discharge the battery in $1 \mathrm{hr}$ ).

$$
\text { C-Rate }=\frac{C_{\text {cell }}}{I}
$$

The required charge or discharge current for a particular rate is determined by dividing the capacity (in Ah) by the number of hours for the charge or discharge. For a 5 Ah battery, a $2 \mathrm{C}(0.5 \mathrm{hr})$ discharge will require a current of $10 \mathrm{~A}$.

\subsubsection{Li-Ion Battery Heat Generation During Normal Operation}

During charge and discharge, LIBs internally generate heat which increases the temperature of the cell if it is not appropriately dissipated. In small LIBs, such as a cell phone or laptop battery, the internal heat generation can easily be dissipated to the surrounding environment simply due to their small form factor and their low rates of charge and discharge. Large LIBs, such as those found in an $\mathrm{EV}$, internal heat generation and appropriate dissipation is a significant design consideration for successful and safe implementation. The electrochemical mechanisms by which heat is internally generated in a LIB is presented in this section.

The rate at which electron-transfer reactions (i.e., redox reactions in a battery) occur is a function of the applied potential, $U$ [29]. The applied potential must either be greater or less than 
the equilibrium potential of the redox couple to drive the reversible reaction in the preferred direction. The Nernst equation is used to describe electrochemically reversible reactions by relating the measured electrode potential, $E$, to the standard reference potential, $E^{0}$, and the thermodynamic state of the electrochemical cell:

$$
E=E^{0}+\frac{R T}{n F} \ln \frac{a_{\mathrm{O}}^{v_{\mathrm{O}}}}{a_{\mathrm{R}}^{v_{\mathrm{R}}}}
$$

where $R$ is the ideal gas constant, $T$ is the temperature of the cell, $n$ is the number of electrons transferred in the redox reaction, $F$ is Faraday's constant, $a$ is the activity of the electrochemically active species, and $v$ is the stoichiometric coefficient of the redox reaction. The activity of the electrochemically active species $i$ can be determined as follows:

$$
a_{\mathrm{i}}=e^{\frac{\mu_{\mathrm{i}}-\mu_{\mathrm{i}}^{\theta}}{R T}}
$$

where $\mu$ is the chemical potential of the electrochemically active species. Chemical potential is an intensive thermodynamic property of the species and is calculated by the Gibbs function. The heat generated by a LIB during normal operation is a function of current generated, applied potential, equilibrium electrode potential, and potential distribution throughout the battery. Bandhauer et al. [3] provided the following equation for volumetric heat generation, $q^{\prime \prime}$, in larger format LIBs that relates these quantities:

$$
q^{\prime \prime \prime}=i^{\prime \prime \prime}\left(U-E-T \frac{\partial U}{\partial T}\right)+\left(\sigma|\nabla \phi|^{2}\right)_{\mathrm{cc}, \mathrm{pos}}+\left(\sigma|\nabla \phi|^{2}\right)_{\mathrm{cc}, \mathrm{neg}}
$$

where $i$ "' is the volumetric current generation of the battery. The quantity $U-E$ is the overpotential of the cell, $\eta$, which is discussed further below. $\frac{\partial U}{\partial T}$ is the entropic heat coefficient, which describes the reversible change in the open circuit potential of the cell as a function of cell 
temperature. $\sigma$ is the electronic conductivity of the current collector. The quantity $\sigma|\nabla \phi|^{2}$ is the is a three-dimensional representation of $\mathrm{I}^{2} \mathrm{R}$ joule heating derived using Ohm's Law, and is calculated by multiplying the electronic conductivity by the square of the divergence of the potential field.

The overpotential in LIBs is a consequence of three independent components:

$$
\eta=\eta_{\Omega}+\eta_{\mathrm{a}}+\eta_{\mathrm{C}}
$$

The first is the overpotential due to the ohmic resistance of the cell, $\eta_{\Omega}$. The potential loss associated with overcoming the ohmic resistance of the cell is seen immediately upon the application of current. The resistance of the cell is directly related to the conductivity of the materials and electrolyte used. The second term in Equation (1.11) is the overpotential due to the charge transfer or activation resistance of the electrochemical processes at the electrolyte-electrode interface, $\eta_{\mathrm{a}}$. This portion of the overpotential is what provides the driving force for charge transfer through the electrical double-layer of the cell. The last is the overpotential due to mass transfer limitations, $\eta_{\mathrm{C}}$. This overpotential is what drives the ions in the direction of the concentration gradient. The final two terms in the volumetric heat generation equation of a LIB are often negligible in smaller format LIBs (cell phone and laptop batteries); however, these terms are significant in larger format LIBs (such as those found in EVs) due to significant concentration of current in the tabs during high rate events.

Larger cells are much more susceptible to the negative effects associated with internal heat generation. Kim et al. [30] modeled the internal heat generation of a $15 \mathrm{Ah}$ LIB as a function of discharge rate. The cell is identical to the one used in the Chevrolet Volt, and the cell dimensions were $19 \mathrm{~cm}$ tall $\times 14.3 \mathrm{~cm}$ wide $\times 0.5 \mathrm{~cm}$ thick. Kim showed at high discharge rates $(5 \mathrm{C})$ the 
battery surface temperature increased $17^{\circ} \mathrm{C}$ in the regions of the battery next to the current collector tabs where the current density is very high, while other regions further away from the current collector tabs saw no temperature increase. Large temperature differences present within the cell illustrate that current thermal management systems are not completely effective. In addition, these temperature differences cycle the active materials of the battery at different rates, which can cause non-uniform aging as the cell is cycled.

\subsection{Fundamental Thermal Limitations of Li-Ion Batteries}

State of the art LIBs are inherently dangerous due to the high flammability of the organic carbonate solvents used in the liquid electrolyte and the thermal instability of the most commonly used inorganic lithium salt, $\mathrm{LiPF}_{6}$. Internally generated heat during cycling must be dissipated with a thermal management system or by heat rejection to the environment. If not, the cell temperature will rise. Once the cell temperature rises to $>50^{\circ} \mathrm{C}$, LIBs see significant performance degradation and can often fail catastrophically.

Bandhauer et al. [3] reviewed the thermal limitations of LIBs. The authors concluded that the most prevalent thermal issues are capacity fade, self-discharge, and thermal runaway. Each of these thermal issues have a different impact on a LIB with varying consequences. A brief review of each is provided here.

Capacity fade has been repeatedly observed in LIBs when the cell temperature increases beyond $50^{\circ} \mathrm{C}$. The solid electrolyte interphase (SEI), a passivating layer formed between the electrode and the electrolyte, is known to be very unstable at higher temperatures. The SEI layer forms upon initial cycling of a LIB and its initial formation is characterized by an irreversible capacity loss when charging and discharging the cell for the first time. This is due to the consumption of lithium ions in the electrolyte that become part of the decomposition products in 
the passivating SEI layer. At higher temperatures, the SEI becomes less stable which causes additional decomposition products to be formed, consuming even more lithium ions. The more lithium ions that are consumed in the decomposition products, the less there are to participate in the redox reactions in the battery. As one study has shown, cycling a LIB at temperatures $>50^{\circ} \mathrm{C}$ can cause significant, irreversible capacity fade up to 51\% [31]. Recently, capacity fade due to increased LIB cell temperature grounded the Solar Impulse 2, a solar-powered plane attempting to fly around the world. The on-board LIBs overheated during ascent resulting in irreversible capacity loss, enough to no longer provide enough power during the nighttime portion of flights [32]. Further discussion on the impact of capacity fade specifically in the Nissan LEAF EV LIB pack is given in Section 2.2.

Self-discharge is characterized by a loss of capacity after being charged and stored for a long period a time (typically several months). When the LIB is then used, its capacity is much less than the capacity it had when it was originally charged. The rate of self-discharge is temperature dependent: higher temperatures equate to higher self-discharge rates. The LIB can be recharged to its original capacity and operate normally. Therefore, self-discharge is not an irreversible performance degradation mechanism over the lifetime of a LIB. However, this thermal issue is extremely relevant in applications in which charged LIBs must be stored for long periods of time and provide useful energy when required. Self-discharge reduces the capacity of a state-of-the-art LIB at a rate of approximately $0.44 \%$ per day when stored at $60^{\circ} \mathrm{C}[33]$.

Capacity fade and self-discharge are relevant thermal concerns with real consequences to the end user. Engineers must understand these limitations and design LIB management systems that mitigate these thermal effects. The final and most critical thermal issue that remains to be addressed is thermal runaway. 


\subsubsection{Thermal Runaway}

Thermal runaway can generally be classified as irreversible cascading exothermic reactions that can spontaneously occur at battery temperatures greater than $80^{\circ} \mathrm{C}$. Wang et al. [34] provided a detailed review of the mechanisms of thermal runaway in LIBs. A brief summary of thermal runaway events is given below:

1. At temperatures as low as $69^{\circ} \mathrm{C}$ the SEI layer on the anode begins to decompose.

2. The SEI components decompose exothermically from $90-120^{\circ} \mathrm{C}$.

3. The liquid carbonate electrolyte readily reacts with intercalated lithium in the exposed anode (due to the decomposed SEI layer) to produce flammable hydrocarbons such as ethane and methane. This typically occurs around $100^{\circ} \mathrm{C}$, but has been observed at temperatures as low as $68^{\circ} \mathrm{C}$.

4. At $130^{\circ} \mathrm{C}$, the polymer separator melts allowing the cathode and the anode to short.

5. The metal oxides of the cathode materials begin to decompose providing oxidizer to the flammable hydrocarbons.

6. If the LIB does not have a pressure relief vent, the cell will explode following the mixing of the flammable hydrocarbons and oxygen.

Steps 1-3 can happen in any particular order, and once thermal runaway is started, it often selfpropagates to complete failure. Thermal runaway is a severe safety concern in all LIB applications. If the LIB temperature is not maintained below $69^{\circ} \mathrm{C}$, the progression to thermal runaway becomes a real possibility.

\subsubsection{High Profile Li-Ion Battery Failures}

The adoption of LIBs particularly in the aviation and automotive industries has been consistently challenged by thermal runaway failures. Some of the thermal runaway failures have 
occurred despite active thermal management systems for the battery pack. Some high profile LIB thermal runaway failures are reviewed here.

The aviation industry has only recently applied LIBs due to the high uncertainty of their thermal stability. Boeing spearheaded the first commercial effort to introduce LIBs into airplanes, requiring the FAA to develop safety regulations to approve the use of LIBs. Boeing's 787 was the first commercial airliner to use LIBs, and flew without incident from October 2011 to January 2013. On January 7, 2013, the Auxiliary Power Unit (APU) in a Japan Airlines Boeing 787 Dreamliner caught fire while the plane was parked at the gate of Logan International Airport in Boston, MA [35]. No one was injured during the incident. The APU consists of 8-75 Ah graphite/LCO cells (Figure 1-9). The NTSB final report [35] concluded the fire was caused by thermal runaway in the APU battery. The incident grounded the entire 787 fleet for three months while Boeing battery engineers designed and implemented FAA-approved APU modifications. The original design of the APU and FAA-approved modifications do not contain any active thermal management system. Boeing's analysis of the failure concluded that the only possible trigger for thermal runaway in the APU battery was overcharging. Yet, the NTSB final report states no overcharging was observed by the battery monitoring units leading up to the time of the thermal runaway event. Therefore, the true failure mode of the APU was never identified. True to their
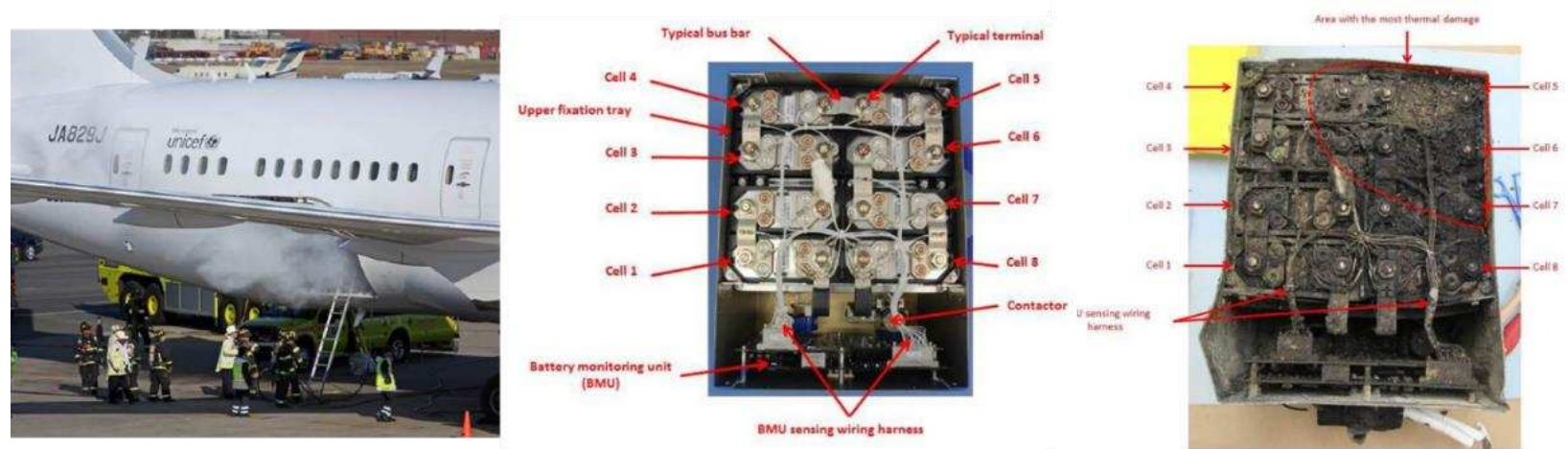

Figure 1-9: Boeing APU Thermal Runaway (Left: Fire from APU; Middle: New APU; Right: Failed APU) [35] 
analysis, Boeing's solution is primarily focused on preventing overcharging by reducing the battery voltage limits and reducing the rate at which the APU is charged. In addition, the solution introduces a $68 \mathrm{~kg}$ stainless steel containment box for the $28 \mathrm{~kg}$ APU and installs a dedicated vent line to the belly of the aircraft in the event that thermal runaway does occur. Full details of the approved solution are provided by Mike Sinnett, the Vice President and Chief Product Engineer at Boeing [36].

In addition to the aviation industry, LIBs are also becoming much more prevalent in the automotive industry. The automotive industry has seen a surge in consumer interest to purchase fully-electric and hybrid-electric vehicles (EVs and HEVs) to reduce dependency on oil and avoid the corresponding dramatic market fluctuations with oil prices. HEVs is a very broad classification of vehicles. Vehicles like the Chevrolet Volt, commonly thought of as an EV, are more correctly termed a series HEV. Series HEVs are powered only by the electric drivetrain. This means the LIB pack is capable of providing enough power to fully propel the vehicle. Series HEVs also contain a small IC engine which acts a generator to charge the LIB pack or to directly power the electric motor. The addition of the IC engine extends the range of the vehicle and dramatically lessens the energy storage requirements of the battery pack [5]. The 2017 Chevrolet Volt uses a $1.5 \mathrm{~L}$ gasoline engine in tandem with an $18.4 \mathrm{kWh}$ LIB pack that enables up to 53 miles of battery-only driving and a further 367 miles of range on gas.

The application of LIBs in HEVs and EVs has even more challenges than Boeing experienced with its APU. Namely, the energy requirements of battery packs are significantly larger. The HEV Chevrolet Volt LIB pack has an energy capacity of $18.4 \mathrm{kWh}$. The EV Tesla Model S LIB pack can be configured to have an energy capacity up to $90 \mathrm{kWh}$. By comparison, the energy capacity of Boeing's APU is $2.22 \mathrm{kWh}$. The greater energy requirement for EVs 
necessitates larger battery packs. Larger packs consist of hundreds or even thousands of individual LIB cells that must be properly managed to minimize the risk of thermal runaway and maintain the longevity of the pack.

Despite the massive engineering challenge, the Chevrolet Volt and Tesla Model S are two examples of engineering successes in implementing LIB packs in EVs. These vehicles have both been rated 5 stars for overall safety by the National Highway Traffic Safety Administration (NHTSA) for all years that the car has been manufactured. The high safety ratings arise mainly from the necessary protection for the LIB pack powering the vehicle. This entails significant chassis reinforcement to prevent damage of the pack during car accidents. Tesla has gone so far as to protect its LIB pack with a ballistic shield [37]. In addition, both of these vehicles employ sophisticated thermal management systems for the battery pack. A detailed review of current thermal management systems for vehicle LIB packs is given in Chapter 2.

However, both the Chevrolet Volt [39] and Tesla Model S [40] have experienced LIB fires due to thermal runaway. A thermal runaway event in the Chevrolet Volt occurred while a 2011 version of the vehicle was parked outside of the NHSTA's crash testing facility. The vehicle had undergone side-impact crash testing three weeks prior to the incident leaving the plastic casing of the LIB pack with minor damage. There were no initial signs of thermal runaway immediately after the side-impact crash testing was performed. Figure 1-10 shows the various event stages

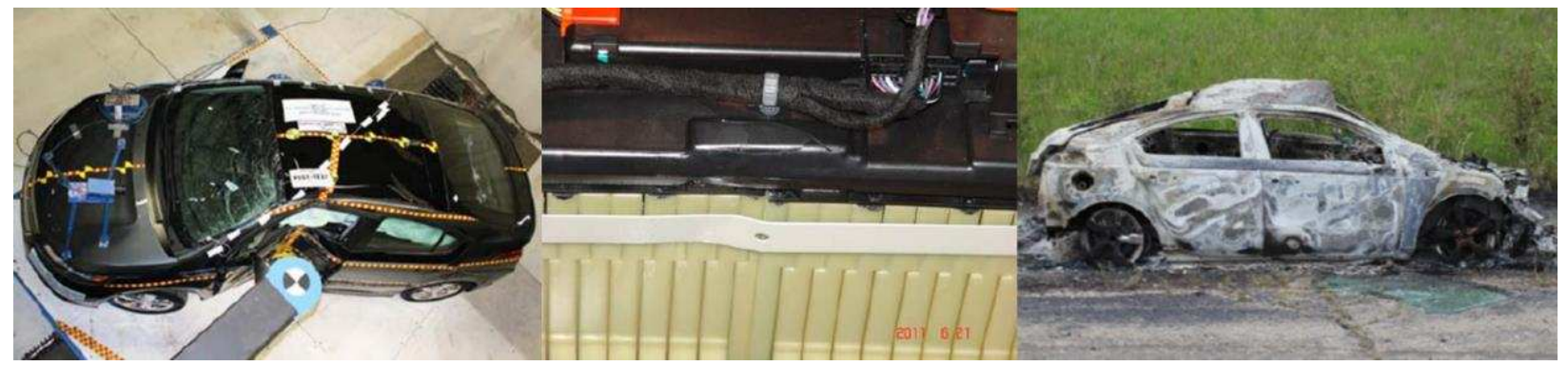

Figure 1-10: Chevrolet Volt Event Series (Left: Side-Impact Test, Middle: LIB Pack Damage, Right: Post Thermal Runaway Event) [38] 
leading up to the thermal runaway event. NHSTA's final report [38] indicated that the transverse stiffener located under the driver's seat had penetrated the tunnel section of the battery compartment during the side-impact crash test, damaging the LIB cells. This damage was attributed to causing the thermal runaway event. NHSTA was unable to replicate the thermal runaway event in four other similar crash tests and the event was considered to be isolated.

A Tesla Model S thermal runaway event occurred after the LIB pack was penetrated by unidentified road debris while travelling at highway speeds. Elon Musk, CEO of Tesla, addressed the incident in a Tesla blog post [41], and provided details of the thermal runaway event. After initial penetration of the LIB pack, the onboard alert system directed the driver to stop and depart the vehicle. The vehicle drove approximately 0.8 miles before the driver was able to stop and exit the vehicle without injury. At this time thermal runaway occurred in the front battery module. Internal fire walls within the pack limited the fire to only the compromised front modules (there are 16 total battery modules that comprise the pack). NHTSA's investigation of the Tesla Model S LIB fire concluded that the incidents were isolated and were not caused by a defect in the car's design [42]. Since the incident, Tesla has added further undercarriage protection of the LIB and increased the default vehicle ride height at highway speeds [43].

In both instances of the LIB thermal runaway failures in these vehicles, the cells within the pack were damaged by external penetration. Countless other LIB failures have occurred due to a multitude of different reasons including poor thermal management [32], poor charger design resulting in overcharging [44], and poor cell manufacturing [45]. Additional LIB thermal runaway failures are discussed in a report released by the National Fire Protection Association [46]. Adoption of LIBs in both the aviation and automotive industries will continue to increase, and the challenge to minimize the potential for thermal runaway may also persist. In addition to the 
structural protection of the LIB pack, significant consideration needs to be given to the design of the battery thermal management system as well as the battery monitoring system. The physical design of LIB cells is also a paramount design consideration as this determines the heat transfer properties such as the thermal resistance. Thermal resistance is the temperature drop that will occur across a body when a certain amount of heat is conducted through it. Therefore, a body that has thermal resistance will require a temperature gradient to conduct the heat. This is of particular concern for LIB design, as the thermal gradients can become so large that temperatures within the cell can increase past reversible use temperatures $\left(>50^{\circ} \mathrm{C}\right)$. An overview of the thermal resistance properties of two different, commercially produced LIBs is presented in Section 2.1.

\subsection{Thesis Organization}

In the following chapters, the motivation, design, and experimental validation of a multifunctional electrolyte (MFE) for the internal thermal management of a LIB is presented. The thermal and electrochemical performance of the MFE is characterized through non-boiling and boiling electrochemical experiments. The resulting data prove the feasibility of the proposed internal TMS that relies on the MFE.

Chapter Two presents a review of the literature on current efforts to manage the thermal limitations of LIB. First, the thermal resistance of a LIB is discussed with two commercial cell examples. Second, current TMSs employed in electric vehicles (EVs) are discussed. Third, previous research efforts to modify the electrolyte of a LIB to improve the thermal stability are reviewed. The literature review highlights the novelty of using a MFE in the proposed internal TMS. Chapter Three describes the proposed internal TMS and the requirements for its proper function. This includes the required LIB cell modifications to enable the TMS and a detailed list of electrochemical and thermal requirements of the MFE. The proposed MFE components are also 
presented, which include a volatile co-solvent, carbonate co-solvent, and lithium salt to form the MFE mixture. Chapter Four contains the non-boiling experimental results that characterize the electrochemical performance of the MFE. Relevant theory, setup, and procedures are given for every experiment performed. All results of the MFE are directly compared to a conventional electrolyte mixture to appropriately assess the electrochemical performance impact of the novel MFE mixture on a LIB. Chapter Five presents the boiling experimental results used to evaluate the thermal and electrochemical performance of the MFE under extreme thermal abuse. To enable this experiment, a custom electrolyte boiling facility was constructed. The design, fabrication, and experimental capability of the boiling facility are discussed. Chapter Six provides concluding remarks and recommendations for future work. Although the feasibility of the proposed internal TMS was proven, the MFE mixture is far from achieving its optimal electrochemical performance. Suggestions for MFE refinement and further validation of the internal TMS are given. Finally, the appendices provide supplemental information on the material preparation procedures used for the experiments performed in this work. Specifically, the electrolyte solvent degassing procedure, cyclic voltammetry working electrode polishing procedure, the slurry-based electrode coating procedure, electrolyte boiling facility component list, and details of thermocouple calibration for the electrolyte boiling facility are given. 


\section{CHAPTER 2. LITERATURE REVIEW}

Research efforts on advancing LIBs are divided into two fields: (1) increasing the performance and (2) improving the safety and implementation. To increase performance, scientists and engineers modify the cathode, anode, and electrolyte chemistry to enable higher cell voltage, specific energy, and energy density than the state-of-the-art LIBs. To improve safety and implementation, investigators work to design more effective thermal management solutions, create more accurate thermal-electrochemical models, and modify the state-of-the-art battery chemistries to have better safety with minimal impact on performance. In this chapter, the thermal resistance of a LIB is discussed. Afterward, a review of the literature for LIB thermal management systems is presented. Furthermore, because the current investigation focuses on new multi-functional electrolytes, prior work by various investigators to modify electrolyte compositions to improve safety of LIBs is also discussed. Deficiencies in the literature are then identified, followed by the summary of the approach taken in the current investigation to address these limitations.

\subsection{Thermal Resistance of a LIB}

LIBs can be manufactured in many different formats. Large battery packs typically consist of cylindrical (e.g., 18650 and 26650 formats), prismatic, or pouch cell designs. Cylindrical format LIBs with numbers 18650 and 26650 correspond to the size of cylindrical can that contains the battery materials. For example, the diameter is $18 \mathrm{~mm}$ and the length is $65.0 \mathrm{~mm}$ for an 18650 cell. Cylindrical cells are the most easily manufactured and one of the most common formats of LIBs. Tesla utilizes $6000+18650$ format graphite/NCA cells in a single pack to power its EVs. Single strips of the anode and cathode electrodes along with separator are wound simultaneously to form a cylindrical jelly roll. The cylindrical jelly roll is then sealed in a steel cylindrical can. Prismatic cells are similar to cylindrical, but can take many different forms and offer varying stored energy 
capacities. The electrodes and separator are typically wound with a specified minimum width producing more rectangular jelly rolls than cylindrical cells. In addition, larger format prismatic cells house their jelly rolls in aluminum or stainless steel cans that are often rectangular in shape. (Boeing's 75 Ah cell utilizes this design.) Pouch cells are often a preferred LIB cell architecture due to their high packaging efficiencies which can approach 90-95\% [47]. Packaging efficiency is defined as the volume of battery materials divided by the total volume of the packaged battery. Pouch cells exhibit a stacked electrode architecture: sheets of electrode material are successively stacked on top of one another in the cell. A vacuum-sealed pouch encloses the electrode stack with minimal volume addition. The Nissan LEAF and Chevrolet Volt LIBs utilize a pouch cell format in their packs.

The cylindrical, prismatic, and pouch cell designs offer very different heat transfer characteristics. One of the key parameters in a conduction heat transfer analysis is the thermal resistance. In all current $\mathrm{LIB}$ architectures, heat is rejected from the external surface of the cell. Figure 2-1 shows a picture of the external thermal management system (TMS) in a Tesla $85 \mathrm{kWh}$ LIB pack. A coolant ribbon is in contact with

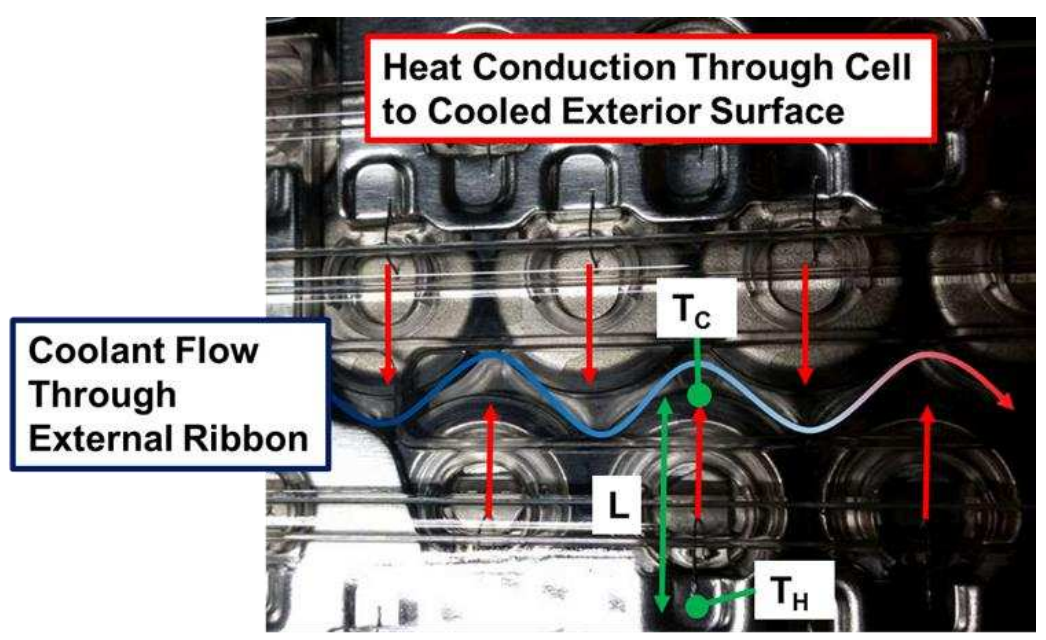
Figure 2-1: Tesla 85 kWh External TMS, Adapted from [48] the exterior surface of every cell within the pack. Internally generated heat within the cell is conducted to the cooled exterior, generating a thermal gradient through the thickness of the cell. 
As an illustrative example, conduction without internal heat generation is formally described by Fourier's Law in rectilinear coordinates as follows:

$$
q_{x}=k A\left(-\frac{d T}{d x}\right)
$$

The temperature gradient $-d T / d x$ indicates the heat flows only from high temperatures to low temperatures. Accordingly, for heat to travel to the exterior of the cell, a temperature gradient must be developed within the battery. The magnitude of the temperature gradient through the thickness of the battery is inversely proportional to the thermal conductivity, $k$. As a result, a low thermal conductivity will produce a high temperature gradient. The materials used to manufacture LIBs have poor thermal conductivity, which produce high temperature gradients within the cell and can lead to undesired high temperatures in the most insulated portions of the cell.

To illustrate the high conduction thermal resistance of a LIB, the equivalent throughthickness $\left(R_{\text {cond }} \perp\right)$ and through-length $\left(R_{\text {cond }} \|\right)$ conduction thermal resistances of the 15 Ah LIB pouch cell in the Chevrolet Volt is compared to that of a block of pure aluminum of equal area and

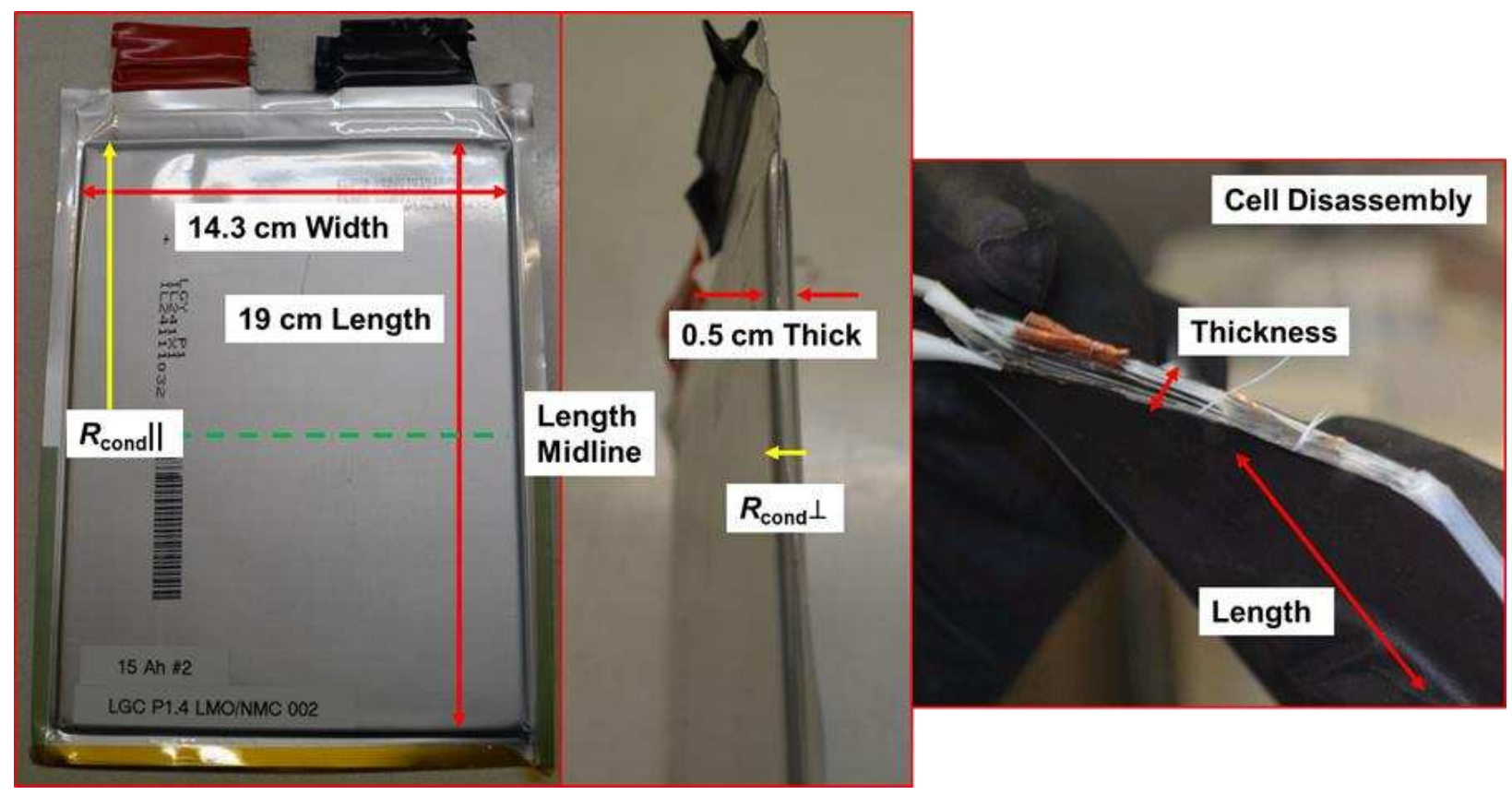

Figure 2-2: Chevrolet Volt 15 Ah LIB 
thickness as the cell (Figure 2-2). The data for thickness, length, width, and number of components in the cell stack in Table 2-1 was obtained by disassembling a 15 Ah Chevrolet Volt battery. The thermal conductivity data is from a study performed by Kim et al. [30] on thermal and electrochemical modeling of the 15 Ah Chevrolet Volt battery.

To determine the through-length and through-thickness thermal resistances for this cell, the thermal resistance for each component of the cell stack was calculated as follows:

$$
R_{\text {cond,i }}=\frac{L_{1}}{k_{\mathrm{i}} A}
$$

$L_{\mathrm{i}}$ is the conduction heat transfer length and $A$ is the area through which heat is conducted. When heat is conducted perpendicularly through the cell stack, $L_{\mathrm{i}}$ is the thickness of the component in the stack and $A$ is the product of the cell length and width (Figure 2-3). For example, the perpendicular conduction thermal resistance of the separator in Table 2-1, is determined by dividing its $25 \mu \mathrm{m}$ thickness (the heat conduction length) by its $1 \mathrm{~W} \mathrm{~m}^{-1} \mathrm{~K}^{-1}$ thermal conductivity and area $(0.19 \mathrm{~m}$ length $\times 0.143 \mathrm{~m}$ width). For the 34 separators that are contained within the cell, this equates to a thermal resistance $0.0313 \mathrm{~K} \mathrm{~W}^{-1}$. Furthermore, the parallel conduction thermal resistance of the separator is determined by dividing half the length, $0.095 \mathrm{~m}$ (the heat conduction length), by the same thermal conductivity with a different area ( $0.143 \mathrm{~m}$ width $\times 25 \mu \mathrm{m}$ thickness). For 34 parallel separators, this results in a thermal resistance of $9.033 \times 10^{5} \mathrm{~K} \mathrm{~W}^{-1}$. To calculate the composite conduction thermal resistances for the cell, the following two formulas were used:

$$
\begin{gathered}
R_{\text {cond }} \perp=R_{\text {CuCC }}+R_{\mathrm{G}}+R_{\mathrm{AlCC}}+R_{\mathrm{NMClLMO}}+R_{\text {sep }} \\
R_{\text {cond }} \|^{-1}=R_{\text {CuCC }}{ }^{-1}+R_{\mathrm{G}}^{-1}+R_{\mathrm{AlCC}^{-1}+R_{\mathrm{NMCLMOO}}{ }^{-1}+R_{\text {sep }}{ }^{-1}}
\end{gathered}
$$

The composite through-thickness conduction thermal resistance was calculated using Equation (2.3), which is a series summation of each of the thermal conduction resistances of the cell components in the thickness direction (Figure 2-3). For the 15 Ah cell in the Chevy Volt, the 
composite cell thermal resistance is $0.0587 \mathrm{~K} \mathrm{~W}^{-1}$. In contrast, the through-thickness conduction resistance for an equivalently-sized aluminum plate is $0.000783 \mathrm{~K} \mathrm{~W}^{-1}$, or 75 times less thermal resistance.

For the through-length thermal resistance, it is assumed that heat can be rejected at both the top and bottom of the battery (Figure 2-4). Using Equation (2.4), the thermal resistance for this direction is $3.73 \mathrm{~K} \mathrm{~W}^{-1}$. This high thermal resistance is due to the very small heat conduction area (component width $\times$ thickness) relative to the length that the heat must be conducted to the cooled top or bottom. The through-length conduction resistance for an equivalently-sized aluminum plate is only $0.5515 \mathrm{~K} \mathrm{~W}^{-1}$, or 7 times less thermal resistance. It can be clearly seen that rejecting heat through the cell thickness will reduce the temperature difference for the most insulated portion of the cell to a cooling fluid on the external surface. The through-thickness conduction of internally generated heat is the approach that the Chevrolet Volt TMS utilizes.

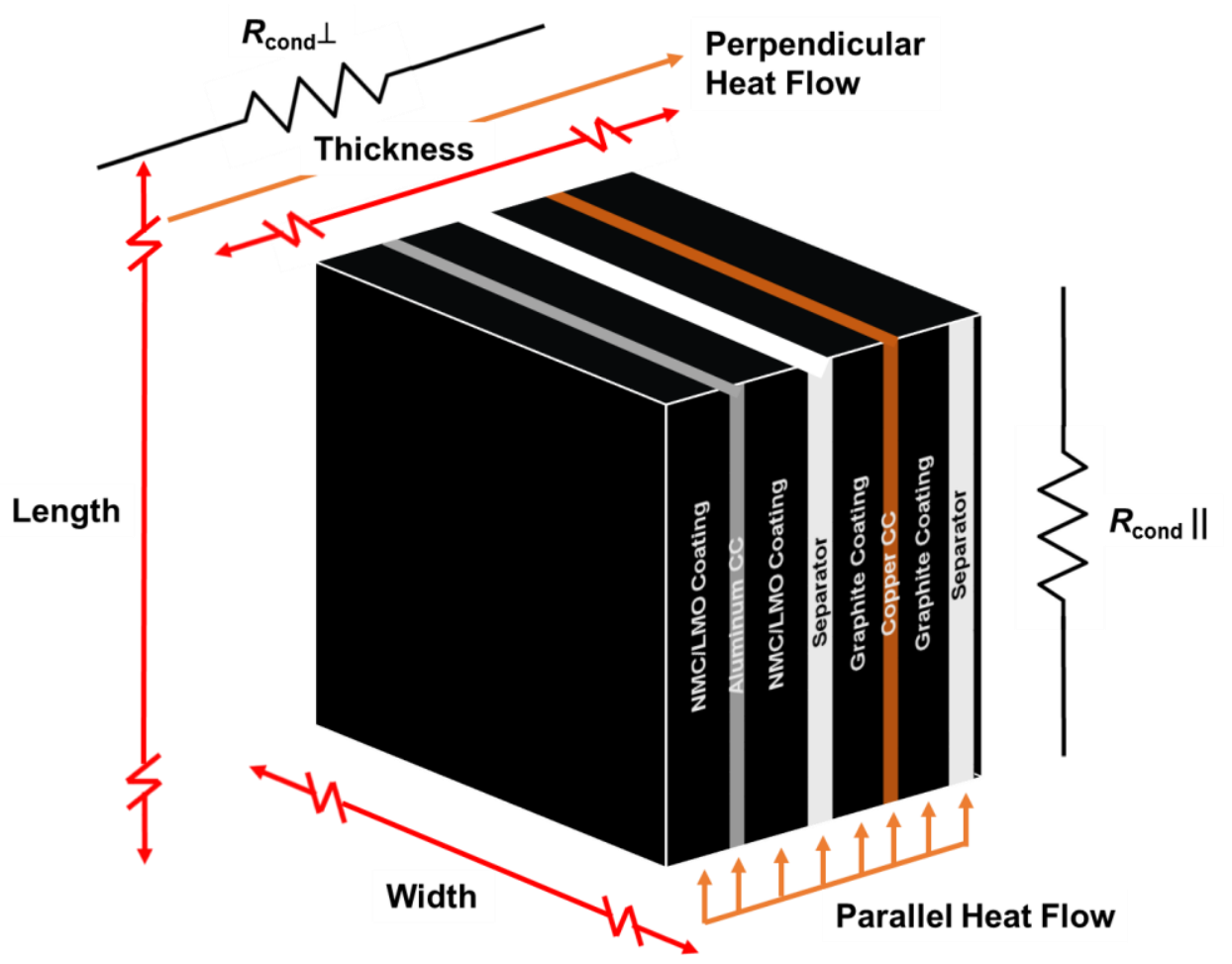

Figure 2-3: Repeating Unit Cell for Calculation of Thermal Resistance of a 15 Ah LIB 
Table 2-1: Thermal Conduction Resistance for Chevrolet Volt 15 Ah Cell

\begin{tabular}{|c|c|c|c|c|c|c|c|}
\hline Component & $\begin{array}{c}\text { Thickness } \\
(\mu \mathrm{m})\end{array}$ & $\begin{array}{c}\text { Thermal } \\
\text { Conductivity } \\
\left(\mathbf{W} \mathbf{m}^{-1} \mathbf{K}^{-1}\right)\end{array}$ & $\begin{array}{l}\text { Length } \\
\text { (m) }\end{array}$ & $\begin{array}{c}\text { Width } \\
\text { (m) }\end{array}$ & $\begin{array}{l}\text { Quantity } \\
\text { in Cell }\end{array}$ & $\begin{array}{r}R_{\text {cond, } \mathrm{i}} \perp \\
\left(\mathrm{K} \mathrm{W}^{-1}\right)\end{array}$ & $\begin{array}{c}R_{\text {cond, i }} \|^{-1} \\
\left(\mathbf{W ~ K}^{-1}\right)\end{array}$ \\
\hline $\begin{array}{l}\text { Copper } \\
\text { Current } \\
\text { Collector }\end{array}$ & 15 & 398 & \multirow{5}{*}{0.19} & \multirow{5}{*}{0.143} & 17 & $2.358 \mathrm{E}-5$ & 0.153 \\
\hline $\begin{array}{l}\text { Graphite } \\
\text { Coating }\end{array}$ & 50 & 5 & & & 34 & 0.0125 & 0.0128 \\
\hline $\begin{array}{l}\text { Aluminum } \\
\text { Current } \\
\text { Collector }\end{array}$ & 15 & 238 & & & 16 & $3.711 \mathrm{E}-5$ & 0.0860 \\
\hline $\begin{array}{l}\text { NMC/LMO } \\
\text { Coating }\end{array}$ & 63 & 5 & & & 32 & 0.0148 & 0.0152 \\
\hline Separator & 25 & 1 & & & 34 & 0.0313 & 0.00128 \\
\hline $\begin{array}{l}\text { Cell } \\
\text { Thickness } \\
\text { (cm) }\end{array}$ & 0.506 & & & & $\begin{array}{c}R_{\text {cond cell }} \\
\left(\mathrm{K} \mathrm{W}^{-1}\right)\end{array}$ & 0.0587 & 3.73 \\
\hline
\end{tabular}

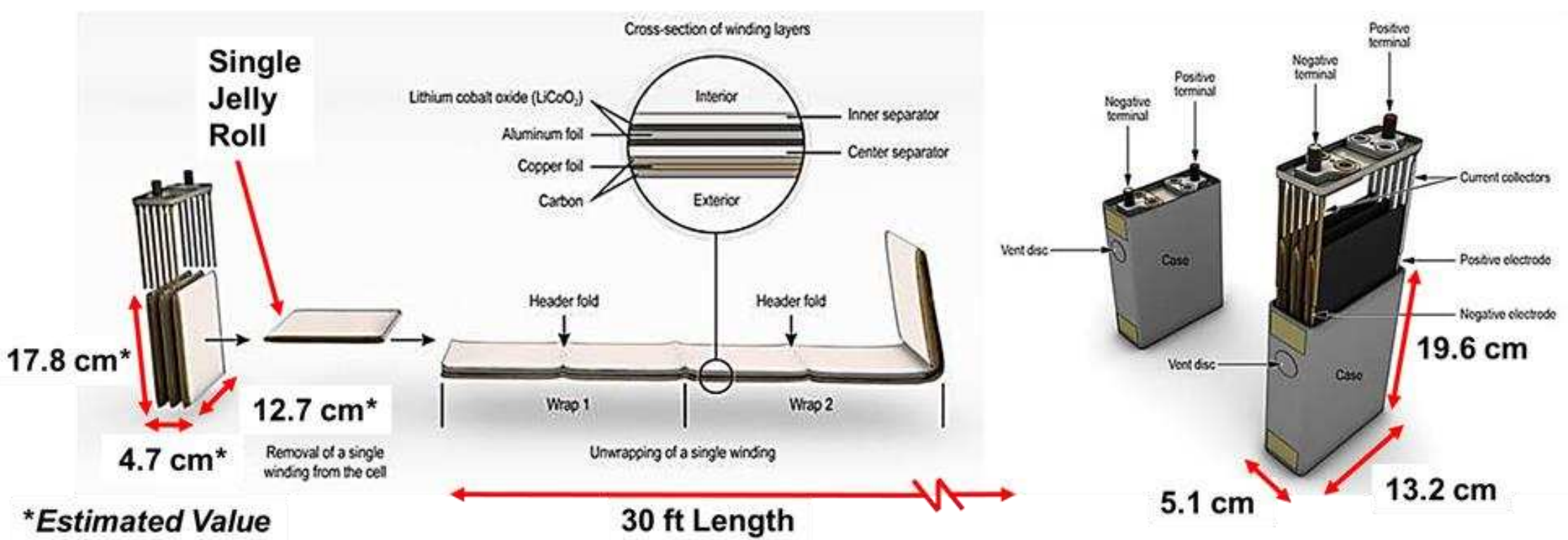

Figure 2-4: Boeing APU 75 Ah Cell Construction Schematic, Adapted from [35] 
The through-thickness conduction thermal resistance becomes even more of a significant issue in larger format prismatic cells, such as the cell used in Boeing's APU (Figure 2-4). Each one of the 75 Ah cells in the APU pack contains three main jelly rolls. Each jelly roll consists of $914.4 \mathrm{~cm}$ of anode, cathode, and separator material wound on itself that must fit within the 19.6 $\mathrm{cm}$ allotted in the height of the casing (Figure 2-4) [35]. Therefore, a conservative estimate of the number of windings required to roll the $914.4 \mathrm{~cm}$ electrode into one of the three main jelly rolls in the cell is 51.4 (i.e., $914.4 \mathrm{~cm} / 17.8 \mathrm{~cm}=51.4$ ), where $17.8 \mathrm{~cm}$ is the folded length. This yields approximately 154 windings per cell. The cell conduction thermal resistance was then calculated using the same procedure described above by approximating each of the windings as a planar stack with length and width of $17.8 \mathrm{~cm}$ and $12.7 \mathrm{~cm}$, respectively. Table 2-2 contains all of the relevant quantities used to determine the thermal resistance of the 75 Ah cell. The through-thickness conduction thermal resistance of the $75 \mathrm{Ah}$ cell is approximately $0.650 \mathrm{~K} \mathrm{~W}^{-1}$ (11 times that of the 15 Ah cell). The through-length conduction thermal resistance was determined to be $0.424 \mathrm{~K} \mathrm{~W}^{-1}$. These calculations assumed the same values of cell component thickness and thermal conductivity

Table 2-2: Thermal Conduction Resistance for Boeing 75 Ah Cell

\section{Thermal}

Thickness Conductivity Length Width Quantity $R_{\text {cond, } \mathrm{i} \perp} \boldsymbol{R}_{\text {cond, } \mathrm{i}} \|^{-1}$

\begin{tabular}{|c|c|c|c|c|c|c|c|}
\hline Component & $(\boldsymbol{\mu m})$ & $\left(\mathbf{W} \mathbf{m}^{-1} \mathbf{K}^{-1}\right)$ & $(\mathbf{m})$ & $(\mathbf{m})$ & in Cell & $\left(\mathbf{K ~ W}^{-1}\right)$ & $\left(\mathbf{W} \mathbf{K}^{-1}\right)$ \\
\hline $\begin{array}{l}\text { Copper } \\
\text { Current } \\
\text { Collector }\end{array}$ & 15 & 398 & \multirow{5}{*}{0.178} & \multirow{5}{*}{0.127} & 154 & $2.570 \mathrm{E}-4$ & 1.31 \\
\hline $\begin{array}{l}\text { Graphite } \\
\text { Coating }\end{array}$ & 50 & 5 & & & 308 & $1.364 \mathrm{E}-1$ & 0.110 \\
\hline $\begin{array}{l}\text { Aluminum } \\
\text { Current } \\
\text { Collector }\end{array}$ & 15 & 238 & & & 154 & $4.298 \mathrm{E}-4$ & 0.785 \\
\hline $\begin{array}{l}\text { NMC/LMO } \\
\text { Coating }\end{array}$ & 63 & 5 & & & 308 & $1.719 \mathrm{E}-1$ & 0.139 \\
\hline Separator & 25 & 1 & & & 308 & $3.410 \mathrm{E}-1$ & 0.0110 \\
\hline $\begin{array}{l}\text { Cell } \\
\text { Thickness } \\
\text { (cm) }\end{array}$ & 4.71 & & & & $\begin{array}{c}\boldsymbol{R}_{\text {cond }} \text { cell } \\
\left(\mathrm{K} \mathrm{W}^{-1}\right)\end{array}$ & 0.650 & 0.424 \\
\hline
\end{tabular}


as the $15 \mathrm{Ah}$ cell. The $75 \mathrm{Ah}$ cells used by Boeing are clearly not designed for removing heat from the internal portions of the cell.

\subsection{State-of-the-Art Thermal Management Systems for Large LIB Packs in EVs}

As consumers continue to adopt EVs, the demand to design more effective TMS for the LIB packs has increased. Effective TMSs are capable of the following: maintaining the temperature of the cells of the LIB pack far below the temperatures at which capacity fade and thermal runaway could occur $\left(50^{\circ} \mathrm{C}\right)$, maintaining a uniform temperature difference $\left(2-5^{\circ} \mathrm{C}\right)$ across all of the cells in a large pack [5], and causing minimal impact on the total size and weight of the pack. TMSs vary dramatically depending upon the EV manufacturer. To illustrate the variety and effectiveness of TMSs in EVs, the Nissan LEAF, Tesla Model S, and Chevrolet Volt will be discussed here.

The Nissan LEAF, first introduced in 2010, contains a $24 \mathrm{kWh}$ LIB pack that is passively cooled by ambient air. The pack architecture (Figure 2-5) shows that minimal attention was given to designing any TMS. The primary method of cooling is by conduction through the aluminum cell modules to the pack case. Heat is ultimately rejected by natural convection to the ambient air [5]. Nissan's design has no method of managing an individual cell's temperature which can vary dramatically depending upon the environment and usage.

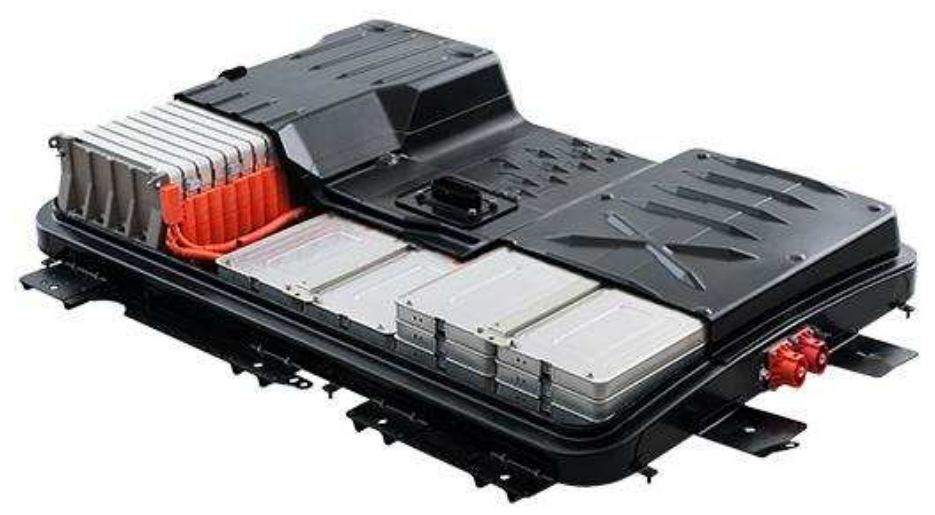

Figure 2-5: Nissan LEAF 24 kWh LIB Pack [49]

The lack of a TMS has not gone unnoticed by consumers. Many LEAF owners have filed complaints citing significant capacity fade in their LIB packs with minimal mileage on the vehicle. Most of complaints have 
originated from owners in the warm climates of Texas and Arizona where cooling by ambient air is not effective due to high ambient temperatures. These owners report that their dashboard battery state-of-charge gauge has fewer illuminating segments after charging which indicates that the pack has lost a sizeable amount of its original stored energy capacity. In one instance of a LEAF driven 29,000 miles, the owner reported 8 capacity segments illuminate out of 12 after charging, which translates to the battery having only $60-66.24 \%$ of its original capacity [50]. Nissan publicly responded to the dissatisfied owners saying that the capacity fade they were experiencing was normal and that all the affected LEAFs were on a "glide path" to $76 \%$ capacity retention after 5 years [51]. On its website, Nissan advertises that the LEAF's LIB pack is covered under factory warranty up to 5 years or 60,000 miles if the capacity degrades to below 9 segments or 66.25 $72.49 \%$ of its original capacity [49].

The capacity fade seen in the Nissan LEAF LIB packs in warm climates should not be a surprise. Pheonix, Arizona has average high temperatures above $40^{\circ} \mathrm{C}$ in the months from June to August [52]. Significant capacity fade due to the SEI decomposing and reforming occurs at cell

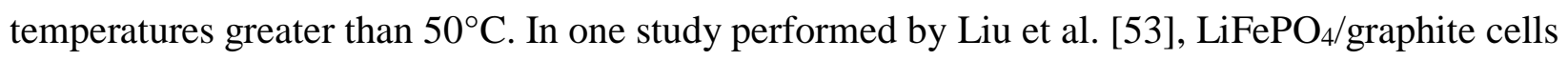
were cycled at high ambient temperatures $\left(60^{\circ} \mathrm{C}\right)$ to find that only $77 \%$ of the cells' original capacity remained after 757 cycles. In comparison, cells cycled at $15^{\circ} \mathrm{C}$ maintained $89 \%$ of their original capacity after 2628 cycles when cycled at the same rate. High cell temperatures cause irreversible capacity loss. The Nissan LEAF utilizes NMC/graphite LIB cells [54], and similar thermally-induced capacity fade has been observed in other LIB cell chemistries [3]. Furthermore, heat transfer from the LEAF battery pack does not occur unless the temperatures inside the cells are higher than the ambient. A cell surface temperature rise of $10-25^{\circ} \mathrm{C}$ has been measured for a $20 \mathrm{Ah}$ cell similarly cooled with $22^{\circ} \mathrm{C}$ air when discharged at rates of 1-4C [55]. The Nissan LEAF 
uses cells with even higher capacity (33.1 Ah), four of which are stacked into a single battery module [56]. This would imply that even under normal use conditions, cell temperatures in the LEAF battery pack could be well above $50^{\circ} \mathrm{C}$. The MIT Technology Review has openly criticized Nissan's design, citing that the lack of a TMS is the primary reason LEAF owners are seeing significant capacity fade in their vehicles [57].

Tesla approached thermal management for its Model S LIB pack in a much different way. The stored energy (up to $90 \mathrm{kWh}$ ) of Tesla's battery pack is larger than any other commercial EV and necessitates effective thermal management. Tesla engineers devised numerous inventions to enable the high energy battery pack. The pack is designed to have active thermal management of every cell (approximately 7,104-18650 cells comprise the $85 \mathrm{kWh}$ pack). Most of the design details of the LIB pack are contained within the intellectual property of Tesla; however, numerous patents have been filed by the company that pertain to the thermal management of its batteries. In one such patent [58], Prilutsky details an active thermal runaway mitigation system that can be used within a LIB pack. The mitigation system utilizes a pressurized fire retardant that is contained within tubes that form a web of coverage over the entire pack. The tubes contain pressure vents that will rupture and spread the fire retardant if the temperature of the pack rises above the maximum

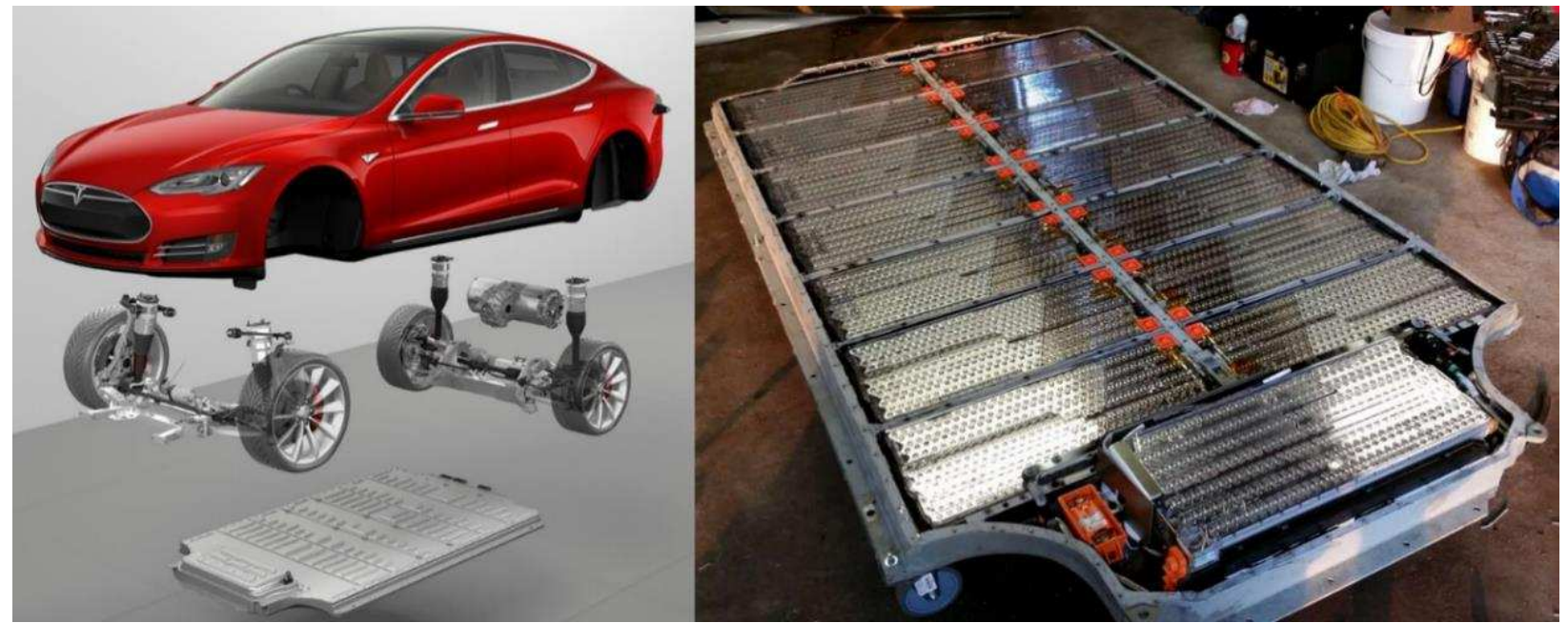

Figure 2-6: Left: Tesla Model S with 85 kWh LIB Pack (Bottom of Image, Source: Tesla.com) Right: Uncovered Pack [48] 
acceptable temperature indicating thermal runaway. Another patent also details a primary TMS for the pack that cycles coolant through a conduit in direct contact with the exterior of the cylindrical cells [59]. The use of this primary TMS

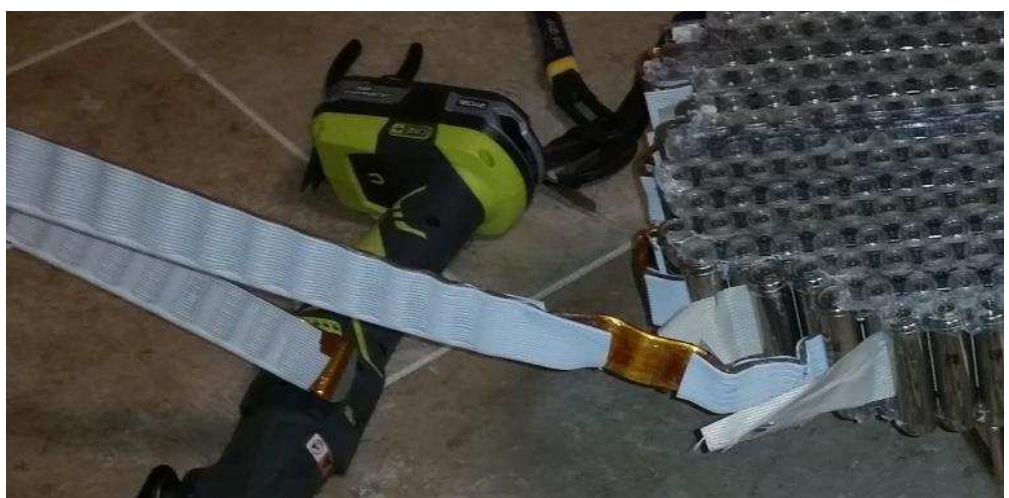

Figure 2-7: Unwound Coolant Conduit from the 18650 Cells of a Tesla $85 \mathrm{kWh}$ LIB Pack [48]

has been confirmed in a Tesla enthusiast blog [48], in which an owner disassembled a $85 \mathrm{kWh}$ battery pack (Figure 2-6 \& Figure 2-7).

The disassembly of the pack showed significant infrastructure was dedicated to ensuring thermal contact of every cell with the cooling conduit. Figure 2-7 shows the strategic placement of the coolant conduit between rows of the 18650 cells. In addition, Tesla owns several patents that describe the use of intumescent materials inside and outside the individual 18650 cells [60, 61]. Intumescent materials are used for passive fire protection: the material swells when exposed to high temperatures. In the event a single cell undergoes thermal runaway, the increase in volume of the intumescent material around the cell provides effective insulation from the adjacent cells. Therefore, catastrophic failure of the entire pack becomes much more unlikely since any thermal runaway failure is isolated to a single cell.

Chevrolet, like Tesla, also utilizes a sophisticated active liquid-cooled TMS to manage the LIB pack in the Volt. Many studies have been performed on the first generation Volt TMS and LIB pack and have been published in a variety of journals. A quick review of the literature shows Chevrolet's design of the individual cells and TMS is much different from Tesla's. Instead of 18650 cylindrical cells which contain about 3.1 Ah of capacity, the Volt utilizes pouch cells that 
contain five times as much capacity (15 Ah) [62]. Consequently, the Volt's pack contains only 288 LIB cells to produce $16.5 \mathrm{kWh}$ of stored energy. The physical shape of the $15 \mathrm{Ah}$ pouch cells (19.3 $\mathrm{cm}$ tall x $14.5 \mathrm{~cm}$ wide $\times 0.5 \mathrm{~cm}$ thick) yields a large heat transfer surface area $\left(279.9 \mathrm{~cm}^{2}\right)$ and minimal conduction heat transfer length $(0.5 \mathrm{~cm})$. Every cell has one side in contact with an aluminum cooling plate. The aluminum cooling plate contains passages for the DEX-Cool coolant (50:50 water/glycol). Kraig Schultz disassembled a Chevrolet Volt battery pack and provided images of cooling plates on his website [63] (Figure 2-8). The coolant is pumped through the plates, absorbs the heat conducted from the cells, and rejects the heat to a variety of heat exchangers external to the pack. Full details of the first generation Chevrolet Volt TMS can be found in a detailed study by Hamut [64] and from General Motors technical papers [21, 62, 65].

The accompanying infrastructure (cooling system, battery management system, and frame) for the Volt battery pack is a significant portion of the total battery system weight. On its website, Chevrolet specifies battery system mass as $190 \mathrm{~kg}$. A single $15 \mathrm{Ah}$ cell (of the 288 cell, $16.5 \mathrm{kWh}$ pack) weighs $0.384 \mathrm{~kg}$. This indicates that approximately $58 \%$ of the battery system mass is actually LIB, with the remainder accounting for the cooling system, battery management system, and frame [21]. This significantly reduces the energy density of the pack. Despite the effectiveness

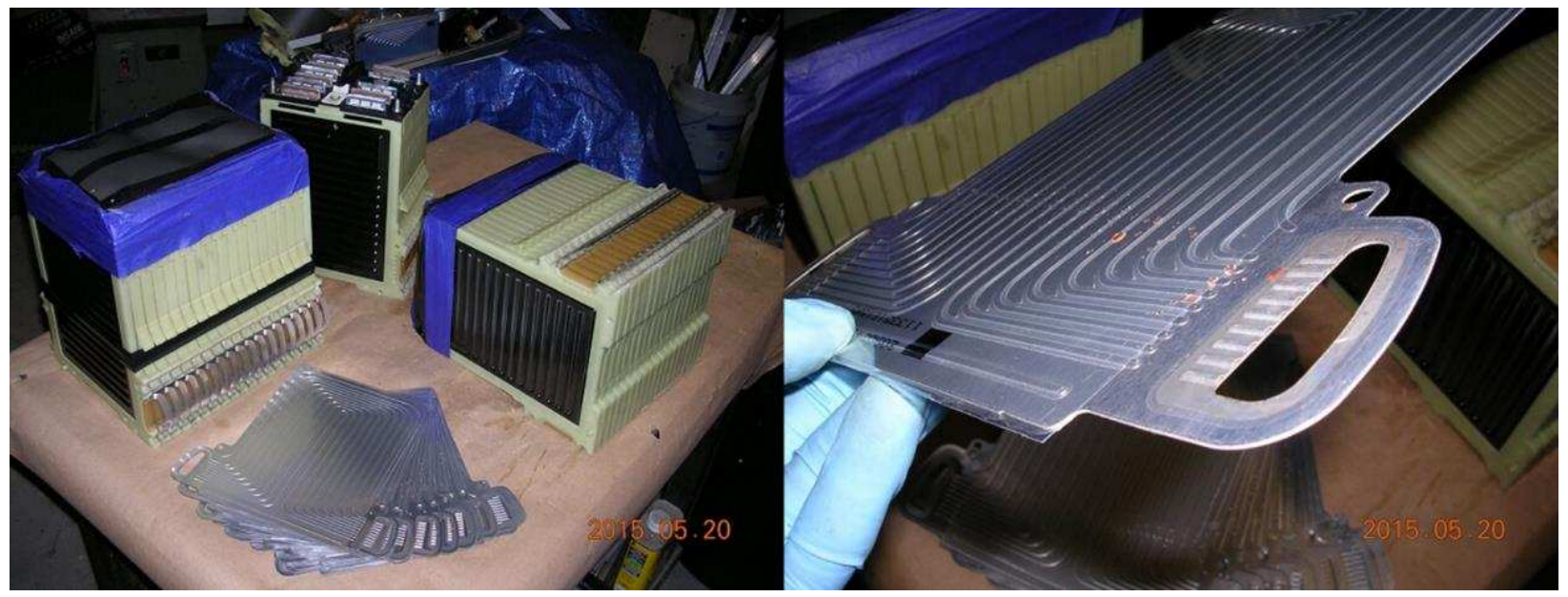

Figure 2-8: Aluminum Cooling Plates Used in Chevrolet Volt Battery Pack [63] 
of the TMS, the size and mass penalty significantly reduces the benefit of using LIB over other battery chemistries.

After the introduction of the Chevrolet Volt in 2010, work to

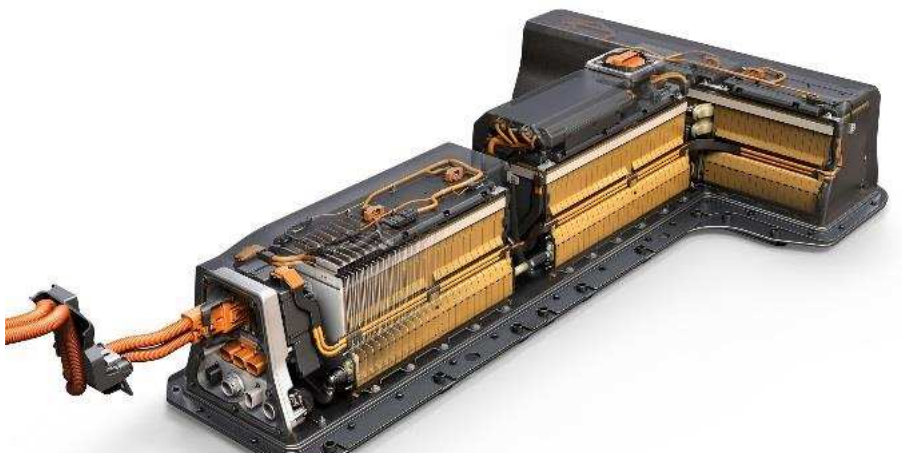
design the second generation Volt battery pack began. The second Figure 2-9: 2016 Chevrolet Volt 18.4 kWh LIB Pack (Source: Chevrolet.com) generation pack will be introduced into the 2016 production vehicle (Figure 2-9). The new pack will have increased stored energy capacity $(18.4 \mathrm{kWh})$, and consist of only 192 total cells each with a capacity of 26 Ah $[65,66]$. The new pack uses the same TMS as the previous iteration.

The Chevrolet Volt TMS has been shown to be effective at managing the battery pack over the lifetime of the vehicle. Idaho National Laboratory (INL) and their testing partner, Intertek, have performed multiple tests on several different Chevrolet Volt battery packs at different stages in its life. One particular 2013 Chevrolet Volt (VIN 3491) showed measured average energy capacity retention of nearly 95\% at 70,776 miles [67]. Another 2013 Chevrolet Volt (VIN 3929) showed measured average energy capacity retention of $96 \%$ at 60,121 miles [68]. The high capacity retention for both of Chevrolet Volt vehicles studied is promising; however, it is unclear the portion of the vehicle mileage that was accrued due to energy exchange from the LIB pack as opposed to the on-board gasoline engine. INL and Intertek have also tested several 2013 Nissan LEAFs. The Nissan LEAF VIN 7885 showed a capacity retention of only $86 \%$ after driving 15,763 miles [69]. The greater capacity fade in the Nissan LEAF compared to the Chevrolet Volt can be attributed to two factors: (1) the Nissan LEAF can only be propelled by its LIB pack and therefore 
Table 2-3: Summary of LIB Packs in Vehicles

\begin{tabular}{|c|c|c|c|c|c|c|}
\hline Vehicle & $\begin{array}{c}\text { Capacity } \\
(\mathbf{k W h})\end{array}$ & $\begin{array}{c}\text { Pack } \\
\text { Mass } \\
(\mathbf{k g}) \\
\end{array}$ & $\begin{array}{c}\text { LIB Mass } \\
\text { (kg), \% of } \\
\text { Total } \\
\end{array}$ & $\begin{array}{c}\text { Pack } \\
\text { Volume } \\
\left(\mathbf{d m}^{3}\right)\end{array}$ & $\begin{array}{c}\text { LIB Volume } \\
\left(\mathbf{d m}^{3}\right), \% \text { of } \\
\text { Total } \\
\end{array}$ & $\begin{array}{c}\text { Cell Heat } \\
\text { Transfer Area } \\
\text { per Volume } \\
\left(\mathbf{d m}^{-1}\right)\end{array}$ \\
\hline $\begin{array}{l}\text { Nissan LEAF } \\
{[70]}\end{array}$ & 24 & 294 & $\begin{array}{c}153 \\
(52 \%) \\
\end{array}$ & $114^{\mathrm{a}}$ & $\begin{array}{c}85 \\
(75 \%) \\
\end{array}$ & $6.1^{\mathrm{b}}$ \\
\hline $\begin{array}{l}\text { Tesla Model S } \\
{[48,71]}\end{array}$ & 85 & 544 & $\begin{array}{c}327^{\mathrm{c}} \\
(60 \%)\end{array}$ & $453^{\mathrm{d}}$ & $\begin{array}{c}118 \\
(26 \%)\end{array}$ & $5.5^{\mathrm{e}}$ \\
\hline $\begin{array}{l}\text { Chevrolet } \\
\text { Volt [21] }\end{array}$ & 16.5 & 190 & $\begin{array}{c}111 \\
(58 \%)\end{array}$ & 138 & $\begin{array}{c}40 \\
(29 \%)\end{array}$ & 19.6 \\
\hline \multicolumn{7}{|c|}{ a LEAF LIB pack volume estimated from dimensions of modules (48 modules total) } \\
\hline \multicolumn{7}{|c|}{$\begin{array}{l}\text { b Heat transfer area estimated by summing all of the surface areas of the rectangular pack dimensions provided by } \\
\text { Nissan }(15.705 \times 11.88 \times 2.649 \mathrm{dm}) \text {; note: this value is for passive air cooling (i.e. no TMS) }\end{array}$} \\
\hline \multicolumn{7}{|c|}{ c Model S LIB mass estimated from specifications of Panasonic Enhanced Nickel/Carbon 18650 cell } \\
\hline \multicolumn{7}{|c|}{$\begin{array}{l}\text { Model S LIB pack volume estimated by approximating module size to be } 3.60 \times 7.74 \times 1.016 \mathrm{dm} \text { (16 modules total) } \\
\text { from pack disassembly images }\end{array}$} \\
\hline \multicolumn{7}{|c|}{${ }^{\mathrm{e}}$ Cooling conduit was assumed to have $90^{\circ}$ of surface area contact with each 18650 cell $\left(0.092 \mathrm{dm}^{2}\right.$ per cell $)$} \\
\hline
\end{tabular}

Although the Volt and Model S cooling systems are effective at mitigating thermal issues in LIB packs for EVs and HEVs, these TMSs still face significant challenges that limit their effectiveness. Liquid cooling systems increase battery pack weight and volume, significantly reducing the volumetric and gravimetric energy densities that set LIB apart from other battery chemistries (Table 2-3). The values presented in Table 2-3 are estimates; further investigation is required to determine more exact values, particularly for pack volume and LIB volume. The liquid cooled TMS in the Tesla Model S and Chevrolet Volt occupy a significant volume of the pack: only $26 \%$ and $29 \%$ of the pack volume are occupied by LIB cells in each of these vehicles, respectively. Again, it is unclear in these estimations as to why the volume fraction of LIB is so low relative to the reported pack volume. The Nissan LEAF's cells occupy $75 \%$ of the pack volume; with the greater percentage due primarily to the lack of a TMS and from the pack volume being estimated by the module size. Of the three packs listed, the Chevrolet Volt has the greatest 
cell heat transfer area per cell volume. This indicates that the Volt TMS has a much greater capacity to limit temperature differences within the cell whereas the Model S TMS is more restricted due to a decreased surface area to volume ratio. In addition, the coolant utilized by the TMSs increases in temperature as it is cycled through the pack due to the absorption of heat from the cells. Consequently, heat is removed at a decreasing rate as the coolant travels through the pack. Ensuring equal cooling among the cells is a difficult task with the current liquid cooling approaches. Equal cooling is vital because the more evenly cooled the cells are, the more uniformly the cells will cycle and age [5]. For large LIB packs, this requires that the coolant flow be split among different modules within the pack, or for the coolant to be repeatedly conditioned as it travels through the entirety of the pack, adding significant complexity to the design.

\subsection{Internal Thermal Management of LIBs}

Internal cooling is an alternative approach to the thermal management of LIBs. There are immediate benefits to employing an internal approach, namely much lower thermal gradients within the cell and, if two-phase heat rejection is utilized, negligible temperature rise depending upon the cooling fluid state. However, modifying a LIB to incorporate an internal TMS is very technically difficult, as the internal TMS should have no effect on the electrochemical function. Furthermore, internal TMSs must prove to be advantageous over the current external TMSs by demonstrating cell temperature uniformity and improvements in the gravimetric and volumetric energy density of the pack and TMS. Only a few studies have proposed and investigated internal thermal management strategies for LIBs and are described below. The author was unable to identify any instances of commercially-implemented internal TMSs for LIBs.

Internal cooling for LIBs was first introduced by Bandhauer et al. with the use of R-134a refrigerant hermetically-sealed in microchannels [72]. The microchannels were proposed to be 
embedded into the LIB in between

a split copper current collector or by using a copper current collector with the microchannels prefabricated within (Figure 2-10). The study focused primarily on evaluating the thermalhydraulic performance of R-134a with varying heat inputs at the channel

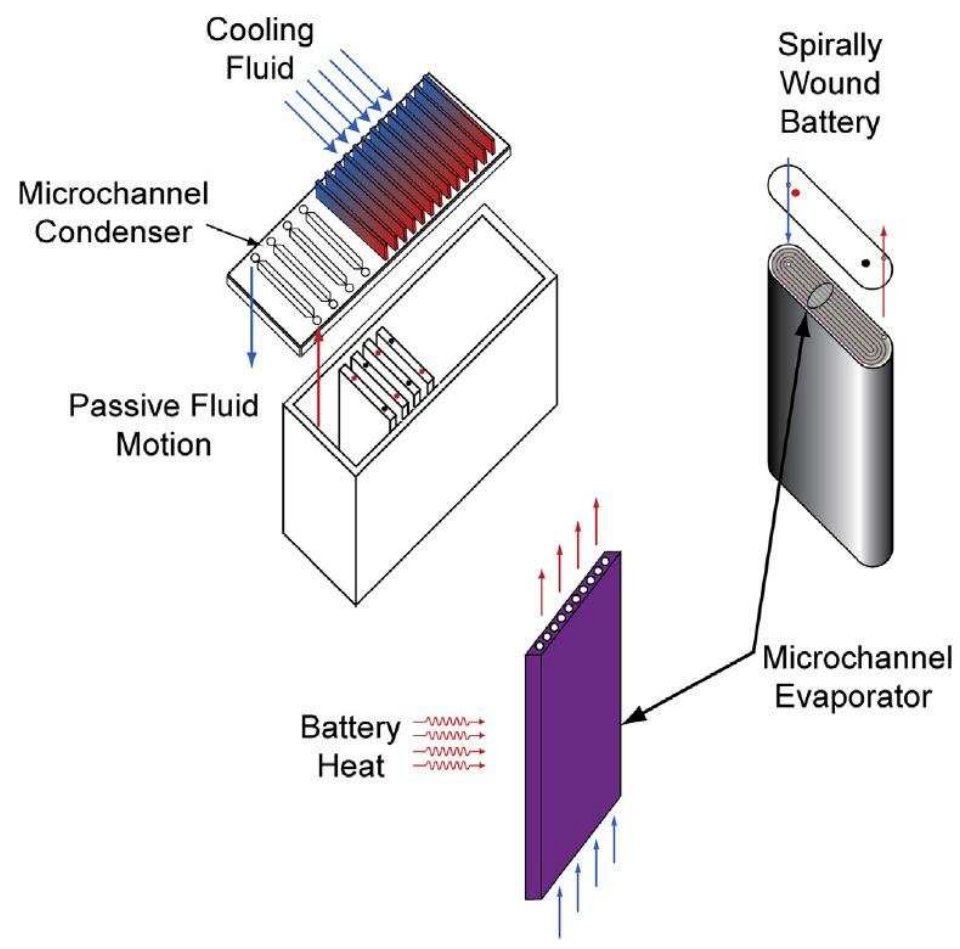

concept in a LIB cell architecture $(3.175 \mathrm{~mm} \times 160 \mu \mathrm{m})$. LIB heat

Figure 2-10: Bandhauer et al. Proposed Internal TMS with Microchannel Evaporator Containing R-134a Refrigerant [72]

generation was simulated using a thin film heater in direct contact with the channels. The results showed that the two-phase refrigerant flow was capable of passively rejecting heat generation up to $6230 \mathrm{~W} \mathrm{~L}^{-1}$. This is a highly promising result as the authors reported that the maximum heat generation of a LIB during a high discharge is at most approximately $200 \mathrm{~W} \mathrm{~L}^{-1}$. These results show that two-phase heat transfer can be an extremely effective means to internally cool a LIB. The study, however, did not investigate the proposed TMS in a LIB.

As opposed to using a refrigerant for the internal TMS, another study describes utilizing the electrolyte as the cooling medium in a LIB. Mohammadian et al. used a computational model to evaluate the impact of cooling a LIB with the electrolyte with small channels created in the positive $(100 \times 90 \mu \mathrm{m})$ and negative electrode $(100 \times 60 \mu \mathrm{m})$ [73]. In the proposed system, the electrolyte is externally pumped through channels within the cell. The model evaluated a single 
unit cell that contained the two cooling channels and compared the thermal performance to liquid cooling on a single exterior surface of the unit cell. The analysis showed the internal cooling channels are capable of maintaining a more uniform cell. For internal cooling, the standard deviation of the temperature field inside the cell was decreased by 3.93 to $5.33 \times$ over external cooling for an internal pumping power of only $0.024 \mathrm{~W}$. However, there are significant deficiencies in the study for the proposed TMS. The authors do not discuss the electrochemical impact of using the electrolyte in the internal TMS, nor is there any description of the external heat exchanger required for cooling the electrolyte. Finally, no description of possible channel fabrication techniques in the positive and negative electrode are provided. Although the study did show a significant advantage to the internal TMS, there is no physical validation to the authors' proposed internal TMS.

Strategies for external and internal cooling for LIB thermal management have been reviewed. In external TMSs, for heat to be removed from the cells, it must first be conducted through the thickness of the LIB. As previously discussed, the low thermal conductivity of the battery materials can lead to high temperature gradients within the battery when heat is conducted to the cooled exterior surface. This limits the geometry of the cell used, as the conduction heat transfer length is a significant consideration. In addition, the heat generation within a larger format LIB is not uniform. Certain portions of the cell, namely the sections closest to the current collector tabs, generate more heat than portions of the cell further away. Internal TMS have been proposed that aim to overcome the primary limitations of external TMS. The lack of experimental verification of internal TMSs in LIBs limits the advancement of the cooling strategy. There is currently no physically-demonstrated TMS that can effectively compensate for the poor thermal conductivity properties of the battery, address the non-uniform heat generation, and passively 
manage every cell's temperature. Because internal cooling strategies are promising means to achieve this, prior studies that have explored modifying the LIB electrolyte are discussed in the next section.

\subsection{Prior LIB Electrolyte Modification Research}

The flammable liquid carbonate electrolyte native to LIBs is one of the most pressing safety concerns that prior investigations have attempted to address. The electrolyte contains two primary constituents: solvents and lithium salt. As mentioned in Section 1.2.3, the solvents used in LIB electrolytes include cyclic (ethylene carbonate, EC) and linear carbonates (dimethyl carbonate, DMC; ethyl methyl carbonate, EMC; diethyl carbonate, DEC). The linear carbonates are inherently extremely flammable. Flammability of volatile substances is typically characterized by determining the flash point. The flash point of a volatile substance is defined as the lowest temperature at which an ignitable mixture can be formed with air. The flash points of DMC, EMC, and DEC are $18.3^{\circ} \mathrm{C}, 23^{\circ} \mathrm{C}$, and $31.1^{\circ} \mathrm{C}$ respectively [46]. These flash points are all well within the use temperatures of a LIB. The lithium salt, $\mathrm{LiPF}_{6}$ in most commercially produced cells, has severe thermal limitations as well. The $\mathrm{PF}_{6}{ }^{-}$anion of the salt is one of the primary reactants in producing electrolyte decomposition products. The six fluorine atoms of a single anion are a very effective oxidant which accelerate the cascading thermal runaway reactions in LIBs. The inorganic anion also readily reacts with water molecules to form toxic substances such as hydrofluoric acid [74].

To date, the research approach to address these thermal limitations has been to modify the current electrolyte mixtures to include substances that suppress or eliminate the flash point of the electrolyte and show reduced reactivity under thermal runaway conditions. Research studies to 
modify LIB electrolytes by the addition of fire retardant additives and other non-flammable fluids (e.g., perfluoropolyethers and hydrofluoroethers) are reviewed here in the next two sections.

\subsubsection{Fire Retardant Co-Solvents}

Fire retardant (FR) additives in LIB electrolytes were reviewed by Nagasubramanian et al. [75]. The authors provided a comprehensive list of FRs that have been investigated to improve the thermal stability of LIB electrolytes by increasing the flash point of the electrolyte. In general, FRs contain phosphorus as the central atom of the molecule. The phosphorus is the basis of organophosphate and organophosphite compounds. The compounds contain alkyl groups, but can easily be synthesized to contain fluorinated groups as well. When fluorine bonds with carbon in these synthesized FRs, the strongest and most thermally-stable bond in organic chemistry is formed [25]. The carbon-fluorine bond is of direct contrast to the thermal instability caused by the inorganic phosphorous-fluorine bonds in the $\mathrm{PF}_{6}^{-}$anion of $\mathrm{LiPF}_{6}$, which greatly reduces the thermal stability of conventional LIB electrolyte mixtures. The combination of fluorine and phosphorus provide the fire suppressing properties desirable in battery electrolytes. Phosphorus radicals are attributed to readily combining with combustion radicals to form stable products. Combustion radicals form when the original fuel's bonds are broken through the interaction with other molecules. In this case, the fuel is the carbonate solvents with low flash points. The formation of combustion radicals dramatically accelerates the combustion process. If the radicals are neutralized by phosphorus, the combustion process is typically halted. Several studies on FR cosolvents in LIB electrolytes are discussed below, and a summary of results is provided in Table $2-4$.

Wang et al. [76] studied trimethyl phosphate (TMP) as a co-solvent in the electrolyte mixture to improve the safety of LIB electrolytes. TMP, a FR in plastics production, was 
investigated due to its hydrogen radical absorption properties - hydrogen radicals are a primary combustion radicals found in organic solvent decomposition. Upon electrochemical characterization of TMP by cyclic voltammetry, the authors found that TMP reductively decomposed on a graphite electrode. The only solution found was to include cyclic carbonate solvents such as propylene carbonate (PC) and EC in the electrolyte, which are known to form stable passivation layers on graphite electrodes. PC and EC have much higher flashpoints than the linear carbonate solvents (135.3 and $163.5^{\circ} \mathrm{C}$, respectively [46]). Therefore, nonflammable binary electrolytes (TMP + PC or EC) were mixed with TMP content as low as $20 \%$. When binary electrolytes were mixed with linear carbonates (DEC and EMC) non-flammability was only achieved with $60 \%$ or greater TMP. In all instances of electrolyte formulations, it was evident that the addition of TMP negatively affected the performance of the cell; the discharge capacity of a 1.0 M LiPF 6 EC:DEC:TMP (60:20:20) cell was approximately $20 \%$ less than that of the $1.0 \mathrm{M}$ $\mathrm{LiPF}_{6} \mathrm{EC}: \mathrm{DEC}$ (50:50) cell with a cycling current density of $0.2 \mathrm{~mA} \mathrm{~cm}^{-2}$. Although the authors conclude that the addition of TMP into the electrolyte improves the thermal stability of LIBs, the performance degradation is unacceptable compared to state-of-the-art electrolyte chemistries.

Kang et al. [77-79] investigated fluoroalkyl phosphates [tris(2,2,2-trifluoroethyl) phosphate, $\quad$ TFP; $\quad$ bis(2,2,2-trifluoroethyl)-methylphosphate, $\quad$ BMP; $\quad$ and $\quad(2,2,2-$ trifluoroethyl)diethyl phosphate, TDP] as candidate nonflammable co-solvents in LIB electrolytes. TFP, BMP, and TDP all have very low dielectric constants and consequent inability to solvate $\mathrm{LiPF}_{6}$ salt by themselves. Therefore, all the investigated candidate electrolytes were formed from mixtures of EC and EMC. Self-extinguishing tests showed a minimum of 20\% TFP or BMP was required in the electrolyte mixture containing equal portions of EC and EMC to achieve nonflammability. TDP achieved non-flammability when it was $\geq 40 \%$ of the electrolyte mixture. The 
ionic conductivity of the candidate electrolyte mixtures was found to decrease linearly with increasing content of TFP or BMP. Further, TFP and BMP were found to have satisfactory interfacial stability on both nickel-oxide cathodes and graphitic anodes. TDP proved unable to form a stable SEI layer on the graphic anode, which the authors attributed to the instability of the partially fluorinated molecule. In full cell tests, TFP and BMP (mixed at a ratio of 15\% with 1.0 $\mathrm{MLiPF}_{6}$ 1:1 EC:EMC) showed high reversibility and comparable capacity retention to the baseline (1.0 $\mathrm{M} \mathrm{LiPF}_{6}$ 1:1 EC:EMC). During high rate cycling tests (up to 2C), electrolytes with higher mixing percentages of TFP showed severely reduced discharge capacity (up to 75\% lower discharge capacity for mixtures containing 40\% TFP). The authors showed rate capability improvements by mixing TFP with a ternary electrolyte mixture of $1.0 \mathrm{M} \mathrm{LiPF}_{6}$ in PC:EC:EMC (1:1:3). The authors concluded that a satisfactorily nonflammable electrolyte mixture was found. In this TFP-based quaternary electrolyte, the discharge capacity at a $2 \mathrm{C}$ rate was reduced by approximately $28 \%$ compared to the baseline organic solvent-based electrolyte.

Zhang et al. [80] investigated tris(2,2,2-trifluoroethyl) phosphite (TTFP) as a candidate nonflammable co-solvent based on the relative success of TFP in the studies performed by Kang et al., particularly in the TFP-based quaternary electrolyte mixture. TTFP is very similar to TFPTTFP contains a lone pair of electrons, whereas TFP utilizes the pair of electrons in a double bond with an additional oxygen atom to form a phosphate group. By performing self-extinguishing tests, the authors determined that a minimum of $15 \%$ TTFP must be present in the baseline electrolyte (1.0 $\mathrm{M} \mathrm{LiPF}_{6} 3: 3: 4 \mathrm{PC} / \mathrm{EC} / \mathrm{EMC}$ ) for the mixture to be nonflammable. However, similar to previous studies, the addition of TTFP decreased the ionic conductivity of the electrolyte. For 15\% TTFP mixed with the baseline electrolyte, the conductivity was approximately $20 \%$ lower than the baseline electrolyte without the TTFP. The authors did see favorable capacity retention when the 
15\% TTFP electrolyte was cycled in a graphite/nickel metal oxide cell compared to the baseline. The cells were not cycled at high rates $\left(0.1 \mathrm{~mA} \mathrm{~cm}{ }^{-2}\right.$, approximately $\mathrm{C} / 4$ rate); therefore, the authors did not observe the associated capacity loss with the reduced ionic conductivity which becomes critical at higher cycling rates. Given the reduction of conductivity observed in the TTFPbased electrolyte was similar to that of the TFP-based electrolyte, a similar reduction of discharge capacity at a $2 \mathrm{C}$ rate would be expected ( $>25 \%$ discharge capacity).

The above instances of electrolyte modification through the introduction of a fire retardant co-solvent show the relative ineffectiveness of the approach. The effectiveness of fire retardant co-solvents to create a nonflammable LIB electrolyte increases only with increasing content of the inert co-solvent. Unfortunately, the performance of the candidate electrolytes decreases significantly as the relative amounts of the FR increases. In all investigated attempts, the fire retardant co-solvent could not function as the only solvent due to its low dielectric constant and relatively high viscosity which rendered it unable to solvate a lithium salt. Even when mixed with conventional carbonate solvents, the cell performance was compromised with the FR co-solvents. The performance degradation is attributed to two primary factors: poor interfacial stability, particularly on graphitic anodes, and low ionic conductivity. The poor interfacial stability is consequent of the SEI decomposition products of the nonflammable co-solvent which form on the surface of the electrodes. In all recorded instances, the use of FR increased the interfacial impedance of the electrode-electrolyte interface, which reduces the realizable energy of the cell. The low ionic conductivity dramatically affects the rate capability of the cell; in all instances, the performance of the cell decreased significantly with increasing rate. The reviewed studies of FRs in LIB electrolytes are summarized in Table 2-4. 
Table 2-4: Summary of Phosphorus-Based Fire Retardant Co-Solvents in LIB Electrolytes

\begin{tabular}{|c|c|c|c|c|c|c|c|}
\hline Study & $\begin{array}{l}\text { FR } \\
\text { Co-Solvent }\end{array}$ & $\begin{array}{l}\text { Chemical } \\
\text { Formula }\end{array}$ & Molecular Structure & $\begin{array}{l}\text { CAS } \\
\text { Number }\end{array}$ & $\begin{array}{l}\text { Baseline } \\
\text { Electrolyte }\end{array}$ & $\begin{array}{l}\text { Nonflammable } \\
\text { Mixing Ratio }\end{array}$ & $\begin{array}{l}\text { Impact on } \\
\text { Performance }\end{array}$ \\
\hline $\begin{array}{l}\text { Wang } \\
\text { et al. } \\
{[76]}\end{array}$ & $\begin{array}{l}\text { Trimethyl } \\
\text { Phosphate } \\
\text { (TMP) }\end{array}$ & $\mathrm{C}_{3} \mathrm{H}_{9} \mathrm{O}_{4} \mathrm{P}$ & 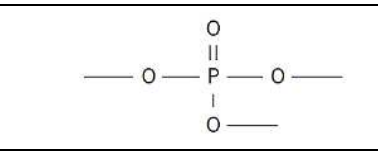 & $512-56-1$ & $\begin{array}{l}1 \mathrm{M} \mathrm{LiPF}_{6} \\
1: 1 \mathrm{EC}^{\mathrm{DEC}}\end{array}$ & $\begin{array}{l}20 \% \text { TMP in } \\
1 \mathrm{M} \mathrm{LiPF}_{6} \\
1: 1 \mathrm{EC} / \mathrm{DEC}\end{array}$ & $\begin{array}{l}20 \% \text { lower discharge } \\
\text { capacity compared to } \\
\text { baseline electrolyte }\end{array}$ \\
\hline \multirow[t]{3}{*}{$\begin{array}{l}\text { Kang } \\
\text { et al. } \\
{[77-79]}\end{array}$} & $\begin{array}{l}\text { Tris(2,2,2- } \\
\text { trifluoroethyl) } \\
\text { Phosphate } \\
\text { (TFP) }\end{array}$ & $\mathrm{C}_{6} \mathrm{H}_{6} \mathrm{~F}_{9} \mathrm{O}_{4} \mathrm{P}$ & & $358-63-4$ & $\begin{array}{l}\text { E1: } 1 \mathrm{M} \\
\mathrm{LiPF}_{6} 1: 1 \\
\text { EC/EMC } \\
\text { E2: } 1 \mathrm{M} \\
\mathrm{LiPF}_{6} 1: 1: 3 \\
\mathrm{PC}_{\mathrm{EC}} / \mathrm{EMC}\end{array}$ & $\begin{array}{l}20 \% \text { TFP in E1 } \\
40 \% \text { TFP in E2 }\end{array}$ & $\begin{array}{l}\text { With } 20 \% \text { TFP in } \\
\text { E1: } 75 \% \text { lower } \\
\text { discharge capacity at } \\
\text { 2C compared to E1 } \\
\text { With } 40 \% \text { TFP in } \\
\text { E2: } 28 \% \text { lower } \\
\text { discharge capacity at } \\
\text { 2C compared to E2 }\end{array}$ \\
\hline & $\begin{array}{l}\text { Bis(2,2,2- } \\
\text { trifluoroethyl) } \\
\text { methyl } \\
\text { phosphate } \\
\text { (BMP) }\end{array}$ & $\mathrm{C}_{5} \mathrm{H}_{7} \mathrm{~F}_{6} \mathrm{O}_{4} \mathrm{P}$ & & $\begin{array}{l}287931- \\
15-1\end{array}$ & E1 & $\begin{array}{l}20 \% \mathrm{BMP} \text { in } \\
\mathrm{E} 1\end{array}$ & $\begin{array}{l}24 \% \text { lower ionic } \\
\text { conductivity; tested } \\
\text { only at C/4 rate }\end{array}$ \\
\hline & $\begin{array}{l}(2,2,2- \\
\text { trifluoroethyl }) \\
\text { Diethyl } \\
\text { Phosphate } \\
\text { (TDP) }\end{array}$ & $\mathrm{C}_{6} \mathrm{H}_{12} \mathrm{~F}_{3} \mathrm{O}_{4} \mathrm{P}$ & & $\begin{array}{l}94080- \\
67-8\end{array}$ & E1 & $\begin{array}{l}40 \% \text { TDP in } \\
\text { E1 }\end{array}$ & $\begin{array}{l}\text { Continual, reductive } \\
\text { decomposition on } \\
\text { graphitic anode; } \\
\text { deemed not feasible } \\
\text { co-solvent }\end{array}$ \\
\hline $\begin{array}{l}\text { Zhang } \\
\text { et al. } \\
{[80]}\end{array}$ & $\begin{array}{l}\text { Tris }(2,2,2- \\
\text { trifluoroethyl) } \\
\text { Phosphite } \\
\text { (TTFP) }\end{array}$ & $\mathrm{C}_{6} \mathrm{H}_{6} \mathrm{~F}_{9} \mathrm{O}_{3} \mathrm{P}$ & & $370-69-4$ & $\begin{array}{l}1 \mathrm{M} \mathrm{LiPF}_{6} \\
3: 3: 4 \\
\mathrm{PC} / \mathrm{EC} / \mathrm{EMC}\end{array}$ & $\begin{array}{l}\text { 15\% TTFP in } \\
1 \mathrm{M} \mathrm{LiPF}_{6} \\
3: 3: 4 \\
\text { PC/EC/EMC }\end{array}$ & $\begin{array}{l}20 \% \text { lower ionic } \\
\text { conductivity than } \\
\text { baseline electrolyte }\end{array}$ \\
\hline
\end{tabular}




\subsubsection{Non-Flammable Fluids as Co-Solvents}

In a continued effort to increase the thermal stability of LIBs, investigators have studied various non-flammable fluids as co-solvents in liquid electrolytes, and sought to mix the fluids with traditional solvents at favorable ratios to produce nonflammable electrolyte mixtures. As opposed to the phosphorous-based fire retardants, these fluids are primarily fluorinated molecules. In this section, two specific groups of heat transfer fluids will be discussed: perfluoropolyethers and hydrofluoroethers. The former has only recently been studied as a LIB electrolyte co-solvent, while the latter has been the focus of several studies dating back to 1999. A summary of the reviewed studies is provided in Table 2-5.

Perfluoropolyethers (PFPE) with molecular weights ranging from 1000-4000 $\mathrm{g} \mathrm{mol}^{-1}$ were investigated as co-solvents in LIB electrolytes in a 2014 study by Wong et al. [81]. PFPEs are long-chained polymers with very low glass transition temperatures enabling them to be liquids at room temperature. These fluids are commercially produced for a variety of heat transfer applications that require an inert working fluid (advertised as Galden HT PFPE by Solvay [82]). PFPEs contain a fluorinated carbon-oxygen backbone which provides inert properties. The authors approached PFPEs as a candidate LIB co-solvent because it is non-flammable (no flash point). PFPEs have a high molecular weight and consequent low dielectric constant, so the authors modified the terminal group of the PFPE molecule to contain a methyl carbonate group, forming PFPE-DMC. With this addition, the PFPE-DMC fluid was capable of solvating LiTFSI salt. The authors found that a LiTFSI salt concentration of approximately $1.0 \mathrm{M}$ produced the highest conductivity mixture. The maximum recorded electrolyte conductivity in the study was achieved with the lowest molecular weight PFPE (PFPE $1000-\mathrm{DMC})$, but it was very low: $0.02 \mathrm{mS} \mathrm{cm}^{-1}$. Only the PFPE $1000-D M C$ candidate electrolyte was tested in lithium/NMC coin cells. The cycling results 
showed poor rate performance compared to the $1.0 \mathrm{M} \mathrm{LiPF}_{6} 1: 2 \mathrm{EC} / \mathrm{DEC}$ baseline electrolyte. At a $\mathrm{C} / 20$ rate, the $\mathrm{PFPE}_{1000}-\mathrm{DMC}$ cell had a reversible capacity that was similar to the baseline. At a $\mathrm{C} / 10$ rate, the reversible capacity of the $\mathrm{PFPE}_{1000}-\mathrm{DMC}$ electrolyte was $20 \%$ less than the baseline. At rates greater than $\mathrm{C} / 10$, the reversible capacity of the $\mathrm{PFPE}_{1000-\mathrm{DMC}}$ cell dropped significantly. At $\mathrm{C} / 8$, the highest cycling rate reported by the authors, the reversible capacity was reduced by an additional $12.5 \%$ from the $\mathrm{C} / 10$ rate. Despite the poor rate performance, the $\mathrm{PFPE}_{1000}-\mathrm{DMC}$ cell did have high charge-discharge efficiency. The authors concluded that a $\mathrm{C} / 8$ rate battery was satisfactory for backing up solar panels and, therefore, the PFPE 1000 -DMC electrolyte is a viable chemistry for a LIB.

Hydrofluoroethers (HFEs) have been studied much more extensively as a LIB electrolyte co-solvent. HFEs were first developed to replace chlorofluorocarbons (CFC) as refrigerants. HFEs have a much lower ozone depletion (i.e., 0) and a relatively low global warming potential compared to CFCs [83]. Juichi Arai pioneered the effort to introduce HFEs in LIB electrolytes as a nonflammable co-solvent. First disclosing his research in a patent [84], Arai later published multiple papers detailing his work [85-87]. Arai sought to design a nonflammable electrolyte that caused a minimal impact on cell performance. His research focused on the HFE-7100 and HFE7200 heat transfer fluids (called MFE and EFE respectively in his study). Arai first determined nonflammable mixing ratios with the HFE co-solvents and EMC. Arai found that increasing the volume percentage of HFE-7100 increased the flash point of the mixed solvents, ultimately producing a no flash point mixture at $80 \%$ HFE-7100. Interestingly, HFE-7200 decreased the flash point of the mixture as its volume percentage increased and did not produce a no flash point mixture at any ratio. Arai attributed the finding to the ratio of fluorine atoms to hydrogen atoms in the HFE-7200 molecule: if this ratio is greater than 2, the mixture is nonflammable. (The F/H ratio 
for HFE-7200 is 1.8 , and is 3 for HFE-7100.) However, the increased fluorination of the HFE molecule that aids its non-flammability significantly decreases the polarity of the molecule and limits its ability to solvate a lithium salt.

Arai extensively studied EMC, DMC, and DEC as co-solvents with HFE-7100 (8:2 HFE7100/co-solvent) and $\mathrm{LiPF}_{6}, \mathrm{LiBF}_{4}$, LiTFSI, LiBETI, and LiFBMSI salts, and found that a $1.0 \mathrm{M}$ LiTFSI 8:2 HFE-7100/EMC electrolyte produced the highest conductivity $\left(1 \mathrm{mS} \mathrm{cm} \mathrm{cm}^{-1}\right)$, with 1.0 M LiBETI EMC electrolyte performing comparably well $\left(0.6 \mathrm{mS} \mathrm{cm} \mathrm{c}^{-1}\right)$. The inorganic lithium salts, $\mathrm{LiPF}_{6}$ and $\mathrm{LiBF}_{4}$, were only solvated to a concentration of $0.2 \mathrm{M}$ in 8:2 HFE-7100/EMC before the salt precipitated out of the electrolyte solution. This was due to the low dielectric constants of the solvents (7.4 for HFE-7100 [88], 2.9 for EMC [89]). Cyclic carbonate solvents such as EC (dielectric constant of 90 [89]) are required to solvate the inorganic lithium salts to high concentrations. Arai then studied the 1.0 M LiBETI 8:2 HFE-7100/EMC in $\mathrm{LiCoO}_{2} /$ graphite 18650 cells. At a C/10 rate, the cell discharged the same capacity as the baseline (1.0 M LiPF $6: 7$ EC/EMC). However, over the course of 100 cycles, the cell capacity faded much more quickly than the baseline. At the end of 100 cycles, $60 \%$ of the original capacity remained, whereas $92 \%$ of the original capacity remained for the baseline. Arai significantly improved the cell's capacity retention with the addition of $\mathrm{EC}(0.5 \mathrm{M})$ and $\mathrm{LiPF}_{6}(0.1 \mathrm{M})$ as additives into the $1.0 \mathrm{M} \mathrm{LiBETI}$ 8:2 HFE-7100/EMC electrolyte. Using the refined electrolyte, the cell maintained $90 \%$ of its original capacity after 560 cycles at a $1 \mathrm{C}$ rate.

Based on the work by Arai, Naoi et al. investigated HFE-7300 and HFE-7600 (referred to as TMMP and TPTP in their study) as an electrolyte co-solvent [90]. HFE-7300 has a higher fluorine to hydrogen ratio (4.3) than HFE-7100 and a theoretically higher fire suppressing ability. Therefore, the authors sought to mix an electrolyte that contained even less of the inert co-solvent 
than the mixtures studied by Arai to further minimize any negative impact on cell cycling performance while maintaining non-flammability. The authors performed flammability tests by providing a sparking ignition source directly above the electrolyte mixture held in a pan in open atmosphere. If the time for ignition exceeded 150 seconds, the mixture was determined to be nonflammable. Confirming their hypothesis, only 50\% HFE-7300 was required to render the candidate electrolyte mixture of 1.0 M LiBETI in 50:5:45 HFE-7300/EC/DEC nonflammable. The authors credited the fire suppressing ability of HFE-7300 to its high vapor pressure compared to its EC and DEC counterparts, assuming the nonflammable HFE-7300 dominated the vapor phase of the mixture. To prove the mixture's feasibility in a LIB, the authors studied the electrochemical stability using cyclic voltammetry (platinum working electrode, lithium counter and reference electrodes). The mixture performed comparably to the baseline electrolytes (R1: 1.0 M LiBETI in 1:1 EC/DEC, and R2: 1.0 M LiBETI in 5:95 EC/DEC) showing satisfactory oxidative and reductive stability from 0 to $4.2 \mathrm{~V}$ vs. $\mathrm{Li} / \mathrm{Li}^{+}$.

The authors then studied the performance of the candidate nonflammable electrolyte versus the two reference electrolytes in a graphite/ $\mathrm{LiCoO}_{2}$ coin cell. The cells were cycled at varying rates, ranging from $1 \mathrm{C}$ to $12 \mathrm{C}$, and at varying temperatures, $-20^{\circ} \mathrm{C}$ to $25^{\circ} \mathrm{C}$. The results clearly showed the candidate electrolyte had the best rate performance $(\sim 50 \%$ greater discharge capacity at a $12 \mathrm{C}$ rate than $\mathrm{R} 2, \sim 66 \%$ greater than $\mathrm{R} 1)$. At $-20^{\circ} \mathrm{C}$, the candidate electrolyte had a discharge capacity ratio (capacity at $-20^{\circ} \mathrm{C}$ divided by the capacity at $25^{\circ} \mathrm{C}$ ) of $60 \%$ compared to $40 \%$ for $\mathrm{R} 2$ and $21 \%$ for R1. Although the results seem extremely encouraging, the reference electrolytes utilized an organic salt, LiBETI, instead of the inorganic salt, $\mathrm{LiPF}_{6}$. The candidate electrolyte is incapable of solvating $\mathrm{LiPF}_{6}$, whereas $\mathrm{R} 1$ and $\mathrm{R} 2$ are capable of solvating high concentrations of 
it. The consequences of choosing the organic salt for the reference electrolytes is an inaccurate comparison to state-of-the-art electrolytes, which exclusively use the inorganic $\mathrm{LiPF}_{6}$ salt.

Naoi et al. continued researching HFEs as co-solvents in LIB electrolytes with HFE-7600 (referred to as TPTP in their study) and compared the performance with their previous work on HFE-7300 [91]. The authors recognized the shortcomings of their previous research effort based on the LiBETI salt and investigated electrolyte mixtures that used $\mathrm{LiPF}_{6}$ in addition to similar mixtures that used LiBETI. HFE-7600 was chosen because the molecule has a higher polarity than HFE-7300 as quantified by their dipole moments: 3.66 Debyes and 2.36 Debyes for HFE-7600 and HFE-7300 respectively. The authors found that electrolytes that contained > 40\% HFE-7600 were nonflammable. Two candidate electrolytes were proposed: E1: 1.0 M LiPF 6 in 5:45:50 EC/DEC/HFE-7600, and E2: 1.0 M LiBETI in 5:45:50 EC/DEC/HFE-7600. Four reference electrolytes were used to compare the performance of the candidate electrolytes: R1: 1.0 M LiPF 6 in 1:1 EC/DEC, R2: 1.0 M LiPF 6 in 5:95 EC/DEC, R3: 1.0 M LiBETI in 1:1 EC/DEC, and R4: 1.0 M LiBETI in 5:95 EC/DEC. Similar to the previous study, graphite/LCO coin cells were used to study the rate capability of the electrolytes. The authors found that the E1-based cell had the lowest discharge capacity compared to cells based on R1 and R2, but had the highest capacity retention (80\%) at high discharge rates (12C), compared to $40 \%$ for $\mathrm{R} 2$ and $20 \%$ for $\mathrm{R} 1$. The authors then attempted to duplicate the rate capability results from their previous study of LiBETIbased electrolytes and were notably unsuccessful. Coin cells with E2 showed discharge capacity retention of $56 \%$ at a $12 \mathrm{C}$ rate, while $\mathrm{R} 3(42 \%)$ and $\mathrm{R} 4(53 \%)$ performed notably better for the same reference electrolyte mixture and active materials. From the previous study, R3 had a discharge capacity retention at a $12 \mathrm{C}$ rate of $25 \%$ and $35 \%$ for $\mathrm{R} 4$. These discrepancies are not insignificant, but the authors attributed them to the greater weight of active material in the coin 
cells used in the previous study. The authors also tested the low temperature performance of the $\mathrm{LiPF}_{6}$-based electrolytes and found $95 \%$ capacity retention at $-20^{\circ} \mathrm{C}$ for $\mathrm{E} 1$, while $\mathrm{R} 1$ saw $21 \%$ and $\mathrm{R} 2$ saw $65 \%$ capacity retention.

The dramatic performance improvements for HFE-based electrolytes reported by Naoi et al. at high rates and low temperatures need further validation. The conflicting results of the performance of the neat-carbonate-based reference electrolytes do not provide an accurate comparison to the performance improvement or degradation as a result of mixing HFE-7300 and HFE-7600 into the electrolyte. Nonetheless, the work by Naoi et al. did show that HFE-7300 and HFE-7600 have the potential to operate as co-solvents in a nonflammable LIB electrolyte.

Based upon the work of Naoi et al., Nagasubramanian and Orendorff thoroughly investigated the thermal stability of Naoi's proposed electrolyte solutions which contained $50 \%$ HFE-7300 and HFE-7600 [92]. Four candidate electrolyte solutions were investigated: E1: 1.0 M $\mathrm{LiPF}_{6}$ in 5:45:50 EC/DEC/HFE-7600, E2: 1.0 M LiBETI in 5:45:50 EC/DEC/HFE-7600, E3: 1.0 M LiTFSI in 5:45:50 EC/DEC/HFE-7600, and E4: 1.0 M LiBETI in 5:45:50 EC/DEC/HFE-7300. Three carbonate electrolytes were utilized as the baseline reference: $\mathrm{R} 1: 1.2 \mathrm{M} \mathrm{LiPF}_{6}$ in $3: 7$ EC/EMC, R2: 1.0 M LiPF 6 in 3:7 EC/EMC, and R3: 1.0 M LiPF 6 in 5:95 EC/DEC. The authors measured the conductivity of the electrolyte solutions from -50 to $50^{\circ} \mathrm{C}$. At every tested temperature the conductivity of the reference carbonate electrolytes were greater than that of the candidate electrolyte solutions. At room temperature, $\mathrm{R} 1$ and $\mathrm{R} 2\left(9 \mathrm{mS} \mathrm{cm}^{-1}\right)$ had a conductivity that was four times greater than that of $\mathrm{E} 2\left(\sim 2 \mathrm{mS} \mathrm{cm}^{-1}\right)$.

Nagasubramanian and Orendorff focused their study on investigating the thermal stability of the electrolytes using accelerating rate calorimetry (ARC) and a novel thermal runaway simulation technique to test electrolyte flammability. The ARC results showed that the electrolytes 
containing the HFE co-solvent with an organic salt (E2, E3, and E4) had the highest thermal stability indicated by the lowest amount of gas volume as the electrolyte samples were heated to well above $450^{\circ} \mathrm{C}$. The primary gas generation in the test occurred at $150^{\circ} \mathrm{C}$ for the carbonate electrolytes, while the HFE electrolytes delayed the gas generation to $220^{\circ} \mathrm{C}$. To test the flammability of the electrolytes, the authors simulated a thermal runaway scenario where $5 \mathrm{~mL}$ of electrolyte was sealed into an empty 18650 cell can and heated until it ruptured. A sparking ignition source was placed directly above the rupture disk to ignite the venting mixture if it is flammable. The results showed that E1-E4 did not ignite while R1 and R3 ignited. R1 ignited within 5 seconds of cell rupture and burned for 6 seconds, while R3 ignited in less than a second and burned for 36 seconds. The higher concentration of the linear carbonate (DEC) in R3 was credited with the reduced ignition time and prolonged burn.

The E2, E4, and R1 electrolytes were then tested in 18650 cells with NMC/graphite. The authors did not specify the rates at which the cells were cycled. The E2 and E4 cells performed almost identically, but still showed $10 \%$ lower discharge capacity than the R1 cell. The cells were also only cycled for a total of 5 charge-discharge cycles. After the cycling tests, the authors then studied the electrolyte gas generation of the 18650 cells containing the E2 and R1 electrolytes using ARC testing. The results showed that the E2 cells had significantly reduced gas generation throughout the temperature ramp to $450^{\circ} \mathrm{C}$ compared to $\mathrm{R} 1$.

The work done by Arai, Naoi et al., and Nagasubramanian and Orendorff have shown that HFE co-solvents in the electrolyte are a feasible option to improve the thermal stability of a LIB. Linear carbonate co-solvents such as DEC, EMC, and DMC can be mixed at any ratio with the HFE fluids. Typical non-flammable mixing ratios with the linear carbonates varied, but were all non-flammable when the HFE fluid contained at least $50 \%$ of the solvent mixture. The lower 
dielectric constant of the HFE fluids as well as the immiscibility in cyclic carbonate solvents prompts the usage of organic lithium salts. Notably, HFE-based electrolytes with LiTFSI and LiBETI organic salts showed acceptable cycling performance despite their lower ionic conductivity (approximately four times less) than state-of-the-art carbonate electrolytes with LiPF6. Arai definitively proved the feasibility of HFE fluids by producing a HFE-7100-based electrolyte mixture that was capable of cycling at a high rate (1C) with a capacity retention greater than $90 \%$ after $500+$ cycles. The results of the studies are summarized in Table 2-5. 
Table 2-5: Summary of Non-Flammable Fluids as Co-Solvents in LIB Electrolytes

\begin{tabular}{|c|c|c|c|c|c|c|c|}
\hline Study & $\begin{array}{l}\text { HT Fluid } \\
\text { Co-Solvent }\end{array}$ & $\begin{array}{l}\text { Chemical } \\
\text { Formula }\end{array}$ & Molecular Structure & $\begin{array}{l}\text { CAS } \\
\text { Number }\end{array}$ & $\begin{array}{l}\text { Baseline } \\
\text { Electrolyte }\end{array}$ & $\begin{array}{l}\text { Nonflammable } \\
\text { Mixing Ratio }\end{array}$ & $\begin{array}{l}\text { Impact on } \\
\text { Performance }\end{array}$ \\
\hline $\begin{array}{l}\text { Wong } \\
\text { et al. } \\
{[81]}\end{array}$ & $\begin{array}{l}\text { DMC } \\
\text { Terminated } \\
\text { Perfluoro- } \\
\text { polyether } \\
\text { (PFPE), } \\
\text { MW = } \\
1000 \mathrm{~g} \mathrm{~mol}^{-1}\end{array}$ & $\begin{array}{l}\text { PFPE- } \\
\text { DMC }\end{array}$ & $\mathrm{H}_{3} \mathrm{C}^{\mathrm{O}} \mathrm{O}_{\mathrm{O}}^{\mathrm{O}-\mathrm{CH}_{2}-\mathrm{CF}_{2}\left(\mathrm{CF}_{2} \mathrm{CF}_{2} \mathrm{O}\right) / \mathrm{m}}$ & $\begin{array}{l}\text { Unique } \\
\text { substance } \\
\text { synthesized } \\
\text { by authors }\end{array}$ & $\begin{array}{l}\text { 1.0 } \mathrm{M} \mathrm{LiPF}_{6} \\
1: 2 \mathrm{EC} / \mathrm{DEC}\end{array}$ & $\begin{array}{l}\text { Intrinsically } \\
\text { nonflammable } \\
\text { with LiTFSI }\end{array}$ & $\begin{array}{l}\text { Low } \\
\text { conductivity, } \\
\text { poor rate } \\
\text { performance } \\
\text { compared to } \\
\text { baseline }\end{array}$ \\
\hline \multirow[t]{2}{*}{$\begin{array}{l}\text { Arai } \\
{[84-87]}\end{array}$} & HFE-7100 & $\mathrm{C}_{5} \mathrm{H}_{3} \mathrm{~F}_{9} \mathrm{O}$ & ${ }_{\mathrm{F}}$ & $163702-07-6$ & $\begin{array}{l}\text { 1.0 } \mathrm{M} \mathrm{LiPF}_{6} \\
3: 7 \mathrm{EC} / \mathrm{EMC}\end{array}$ & $\begin{array}{l}\text { E1: } 1.0 \mathrm{M} \text { LiBETI } \\
8: 2 \mathrm{HFE}- \\
7100 / \mathrm{EMC} \\
\text { E2: E1 + 0.5 M } \\
\mathrm{EC}+0.1 \mathrm{M} \mathrm{LiPF}_{6}\end{array}$ & $\begin{array}{l}\text { E1: High } \\
\text { capacity fade in } \\
\text { cycling tests } \\
\text { E2: } 90 \% \\
\text { capacity } \\
\text { retention after } \\
560 \text { cycles at } \\
1 C\end{array}$ \\
\hline & HFE-7200 & $\mathrm{C}_{6} \mathrm{H}_{5} \mathrm{~F}_{9} \mathrm{O}$ & $\mathrm{F}$ & $163702-05-4$ & & $\begin{array}{l}\text { Flammable at all } \\
\text { mixing ratios due } \\
\text { to } \mathrm{F} / \mathrm{H}<2\end{array}$ & \\
\hline $\begin{array}{l}\text { Naoi et } \\
\text { al. [90] }\end{array}$ & HFE-7300 & $\mathrm{C}_{7} \mathrm{H}_{3} \mathrm{~F}_{13} \mathrm{O}$ & $\mathrm{F}$ & $132182-92-4$ & $\begin{array}{l}\text { R1: } 1.0 \mathrm{M} \\
\text { LiBETI 1:1 } \\
\text { EC/DEC } \\
\text { R2: } 1.0 \mathrm{M} \\
\text { LiBETI 5:95 } \\
\text { EC/DEC }\end{array}$ & $\begin{array}{l}\text { 1.0 M LiBETI } \\
\text { 50:5:45 HFE- } \\
\text { 7300/EC/DEC }\end{array}$ & $\begin{array}{l}\text { Lower } \\
\text { discharge } \\
\text { capacity, higher } \\
\text { capacity } \\
\text { retention at } 12 \mathrm{C} \\
\text { rate, better low } \\
\text { temperature } \\
\text { performance } \\
\text { than R1 and R2 }\end{array}$ \\
\hline $\begin{array}{l}\text { Naga. \& } \\
\text { Oren. } \\
{[92]}\end{array}$ & HFE-7300 & $\mathrm{C}_{7} \mathrm{H}_{3} \mathrm{~F}_{13} \mathrm{O}$ & & & $\begin{array}{l}\text { 1.2 } \mathrm{M} \mathrm{LiPF}_{6} \\
3: 7 \mathrm{EC} / \mathrm{EMC}\end{array}$ & $\begin{array}{l}\text { 1.0 M LiBETI } \\
\text { 5:45:50 } \\
\text { EC/DEC/HFE- } \\
7300\end{array}$ & $\begin{array}{l}\text { Lower } \\
\text { conductivity, } \\
10 \% \text { lower } \\
\text { discharge } \\
\text { capacity than } \\
\text { baseline }\end{array}$ \\
\hline
\end{tabular}




\begin{tabular}{|c|c|c|c|c|c|c|c|}
\hline Study & $\begin{array}{l}\text { HT Fluid } \\
\text { Co-Solvent }\end{array}$ & $\begin{array}{l}\text { Chemical } \\
\text { Formula }\end{array}$ & Molecular Structure & $\begin{array}{l}\text { CAS } \\
\text { Number }\end{array}$ & $\begin{array}{l}\text { Baseline } \\
\text { Electrolyte }\end{array}$ & $\begin{array}{l}\text { Nonflammable } \\
\text { Mixing Ratio }\end{array}$ & $\begin{array}{l}\text { Impact on } \\
\text { Performance }\end{array}$ \\
\hline $\begin{array}{l}\text { Naoi et } \\
\text { al. [91] }\end{array}$ & HFE-7600 & $\mathrm{C}_{8} \mathrm{H}_{6} \mathrm{~F}_{12} \mathrm{O}$ & $\begin{array}{llll}F & F & F & F\end{array}$ & $870778-34-0$ & $\begin{array}{l}\text { R3: } 1.0 \mathrm{M} \\
\mathrm{LiPF}_{6} 1: 1 \\
\mathrm{EC} / \mathrm{DEC} \\
\mathrm{R} 4: 1.0 \mathrm{M} \\
\mathrm{LiPF}_{6} 5: 95 \\
\mathrm{EC} / \mathrm{DEC}\end{array}$ & $\begin{array}{l}\text { E1: } 1.0 \mathrm{M} \mathrm{LiPF}_{6} \\
\text { 5:45:50 } \\
\text { EC/DEC/HFE- } \\
7600 \\
\text { E2: } 1.0 \mathrm{M} \mathrm{LiBETI} \\
\text { 5:45:50 } \\
\text { EC/DEC/HFE- } \\
7600\end{array}$ & $\begin{array}{l}\text { E1: Better low } \\
\text { temperature } \\
\text { performance } \\
\text { than R3 and R4 } \\
\text { E2: Marginally } \\
\text { better high rate } \\
\text { performance } \\
\text { than R1 and R2 }\end{array}$ \\
\hline $\begin{array}{l}\text { Naga. \& } \\
\text { Oren. } \\
\text { [92] }\end{array}$ & HFE-7600 & $\mathrm{C}_{8} \mathrm{H}_{6} \mathrm{~F}_{12} \mathrm{O}$ & & & $\begin{array}{l}1.2 \mathrm{M} \mathrm{LiPF}_{6} \\
3: 7 \mathrm{EC} / \mathrm{EMC}\end{array}$ & $\begin{array}{l}\text { 1.0 M LiBETI } \\
\text { 5:45:50 } \\
\text { EC/DEC/HFE- } \\
7600\end{array}$ & $\begin{array}{l}\text { Similar } \\
\text { conclusions as } \\
\text { HFE-7300, } \\
\text { higher cell } \\
\text { thermal stability }\end{array}$ \\
\hline
\end{tabular}




\subsection{Summary of Deficiencies in Prior Work}

A review of the current thermal management systems (TMSs) in electric vehicles (EVs) has been presented in Section 2.2. The review first investigated the Nissan LEAF LIB pack's TMS which utilizes a passive air-cooled scheme. Nissan's approach has been shown to be ineffective in high temperature climates where the packs are showing significant capacity fade with low mileage on the vehicle. In contrast, the Tesla Model S and Chevrolet Volt have active liquid cooling TMSs. These two TMSs represent the state-of-the-art approach to thermal management in a large LIB pack. The only instances of thermal runaway reported for these vehicles have been caused by external penetration to the pack. Although effective, these TMSs require significant infrastructure within the pack by ensuring the heat transfer medium is in contact with outside surface of every cell, which adds significant weight to the overall system. Furthermore, these TMSs are all external to the cells which lead to higher temperatures in the portions of the cell that are the most insulated.

In Section 2.4, prior research to address the thermal limitations of LIB through electrolyte modification was presented. Specifically, fire-retardants and nonflammable fluids fluids were reviewed as an electrolyte co-solvent. All of the research efforts sought to formulate a nonflammable electrolyte with minimal impact to the cell performance. The phosphorus-based fire retardants reduced the cell performance significantly, rendering the nonflammable characteristics of the electrolyte insignificant. Two classes of other non-flammable fluids were reviewed as electrolyte co-solvents: perfluoropolyethers (PFPEs) and hydrofluoroethers (HFEs). PFPEs were shown to have poor conductivity and consequently poor rate capability compared to state-of-theart carbonate-based electrolytes. HFEs showed much more promise as a feasible nonflammable electrolyte co-solvent. The reviewed works showed that HFEs are capable of cycling at a high rate, 
can be formulated to have high capacity retention and cycle lives, and have good low temperature performance compared to carbonate-based electrolytes.

Several conclusions can be made from the literature review:

- Large LIB packs, such as those in EVs, require a TMS that is capable of keeping the individual cell temperatures of the pack well below $50^{\circ} \mathrm{C}$ and maintain the difference of individual cell temperatures to be within the range of $2-5^{\circ} \mathrm{C}[5]$.

- Passive air cooling is not an effective approach for thermally managing a large LIB pack. The capacity fade seen in the passively, air-cooled Nissan LEAF LIB pack at low vehicle mileage in the warmer climates of Texas and Arizona has led to dissatisfied owners and warranty claims against Nissan $[50,51]$.

- Active liquid cooling is the state-of-the-art approach to thermally managing a large LIB pack, but requires significant infrastructure within the pack to be successfully implemented. This lowers the both the gravimetric and volumetric energy capacity of the pack as it must become larger and heavier to accommodate the TMS. In addition, these TMSs require energy from the LIB pack to operate.

- All current commercial TMSs are external to the cells within the pack. This requires that internally generated heat be conducted through the thickness of the cell to the cooled exterior surface. The conduction of heat produces large thermal gradients within the cell due to the low composite thermal conductivity of the materials used to construct LIBs.

- Modifying the electrolyte with nonflammable co-solvents can be an effective approach to mitigating thermal runaway, depending upon the co-solvent. Most notably, HFE cosolvents show good promise in LIB chemistry. 
- No prior studies have investigated the nonflammable fluids as co-solvents in a LIB for their two-phase heat transfer performance. Furthermore, there have been no investigations of a passive, internal TMS that utilizes a volatile co-solvent within the electrolyte as the working fluid used to absorb and reject internally generated heat. Finally, no prior studies have investigated the physical and electrochemical effects of a boiling electrolyte in a LIB. Investigation of an internal TMS that relies on evaporating a volatile co-solvent is warranted.

\subsection{Focus of Current Investigation}

In the current investigation, a novel approach to lithium-ion battery (LIB) thermal management is investigate to address the fundamental thermal limitation of LIBs: low composite thermal conductivity from the skin of the cells to insulated interior portions of the cell. The proposed internal TMS utilizes a multi-functional electrolyte (MFE), which contains a volatile cosolvent. Upon heat absorption, the volatile co-solvent boils in small channels created in the positive electrode of the LIB at temperatures well below those associated with capacity degradation $\left(<40^{\circ} \mathrm{C}\right)$. The vapor is the condensed on the inside surface of the cell casing and reincorporated into the liquid electrolyte, approximating a loop heat pipe architecture. This system minimizes thermal gradients through the electrode stack by providing localized cooling through the entirety of the LIB, as opposed cooling an exterior cell surface. The candidate MFE mixture is tested for its electrochemical and thermal performance for ultimate use in a passive internal TMS. The specific objectives for the current investigation are:

- Identify candidate volatile co-solvents for lithium-ion electrolytes that meet the electrochemical and thermal requirements of the internal TMS. 
- Identify a candidate carbonate co-solvent and lithium salt to use to formulate the MFE containing the candidate volatile co-solvents.

- Perform non-boiling electrochemical experiments on candidate MFE mixtures including conductivity, electrochemical stability window, half cell and full cell cycling, and impedance spectroscopy to evaluate the impact of the volatile co-solvent on LIB performance.

- Perform electrochemical experiments on candidate MFE mixtures while the volatile cosolvent boils to validate the operation of the internal TMS.

- Use the non-boiling and boiling experimental results of the candidate MFE to inform the future direction of work for the continued validation of the internal TMS. 


\section{CHAPTER 3. CONTINUOUS, PASSIVE INTERNAL COOLING WITH A MULTI- FUNCTIONAL ELECTROLYTE}

The current approach to LIB thermal management is to cool the very outer layers of the electrode stack within the cell. This can cause high thermal gradients through the thickness of the cell, leaving the highest temperatures at the un-cooled center. These thermal gradients can cycle the electrode materials at uneven rates, potentially leading to premature aging in portions of the cell that experience the highest temperatures. Moreover, the highest temperature portions of the cell can experience severe degradation due to SEI decomposition that can ultimately lead to thermal runaway (see Section 1.3.1).

Internal cooling has the potential to completely eliminate these adverse effects. By having the cooling medium in direct contact with the electrodes generating heat, the high thermal resistances that plague conventional external TMSs are eliminated. Furthermore, if the electrolyte can be designed to serve both its electrochemical purpose and partially evaporate to remove heat, a completely passive internal cooling system can be used to cool the normally insulated portions within the cell.

In this chapter, the concept of passive internal cooling with a multi-functional electrolyte (MFE) is described. The required cell modifications, expected battery system impact, and requirements of the MFE are given first. Thereafter, the components of the MFE are described, including candidate volatile and organic carbonate co-solvents and their relevant thermal and electrical properties, as well as the lithium salt. After describing the MFE, the baseline electrolyte used in standard LIBs is described. The performance of the MFE and baseline electrolyte is compared in the next chapter. 


\subsection{Concept Description}

The operating principle for the proposed internal TMS is similar to a closed-loop thermosiphon (Figure 3-1). In a thermosiphon, the working fluid is evaporated upon heat absorption in the evaporator section. Buoyancy forces then propel the vapor from the liquid reservoir into the condenser section, where the vapor is condensed and is transported back to the evaporator [93]. The cooling heat rejection from the condenser is

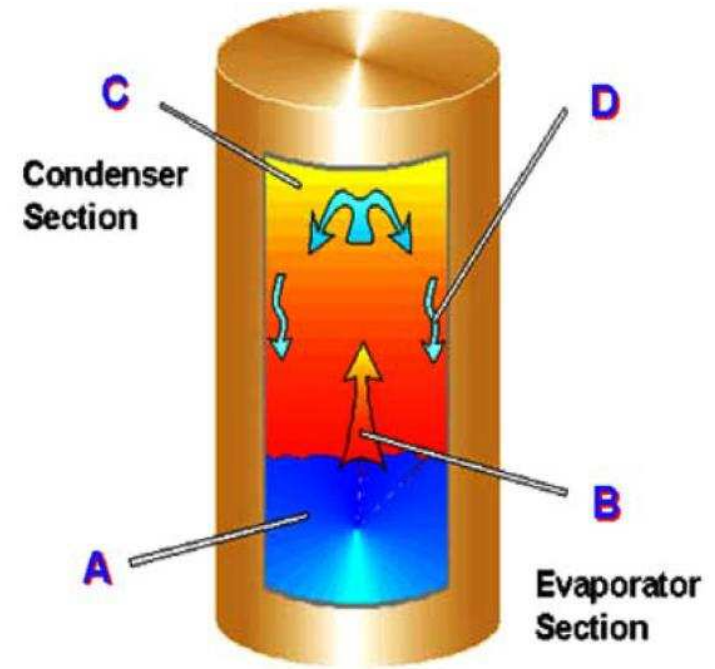

Figure 3-1: Schematic of Two-Phase Closed Loop Thermosiphon [93]

typically forced convection of air or water flowing over the external surface. Thermosiphons are passive devices, which mean that no external pumping of the working fluid is required for the system to operate.

A similar system architecture is proposed for the LIB internal TMS (Figure 3-2). Small channels are created in the positive electrode by the removal of active material in selective locations in the electrode stack. These channels serve as the evaporator section of the thermosiphon. Upon heat absorption, the electrolyte increases in temperature until the most volatile co-solvent undergoes a liquid-vapor phase change. The phase change is a nearly isothermal process that is capable of absorbing a significant amount of heat per unit mass of fluid evaporated. In the current study, the vapor moved to the condenser via buoyancy forces, but it is envisioned that the separator could also serve as a liquid wick similar to that located in a surface-tension driven heat pipe. In Figure 3-2, the condenser can be placed in the thin edge of the LIB cell. Once condensed, the volatile co-solvent is reincorporated into the liquid electrolyte. 


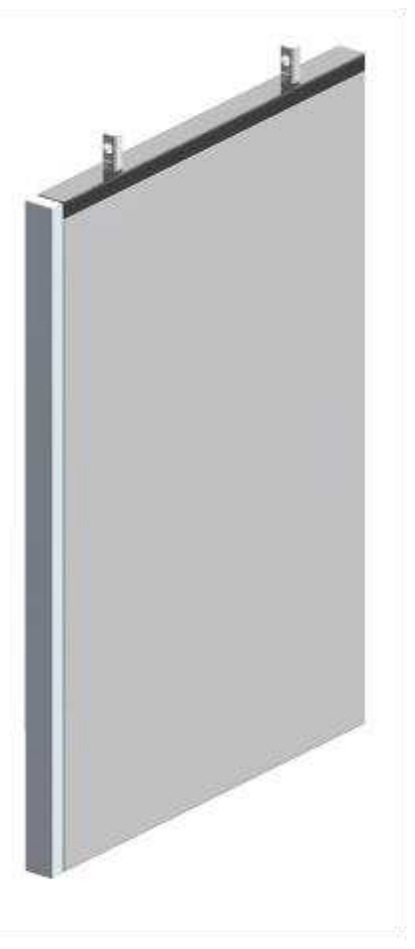

Assembled Cell

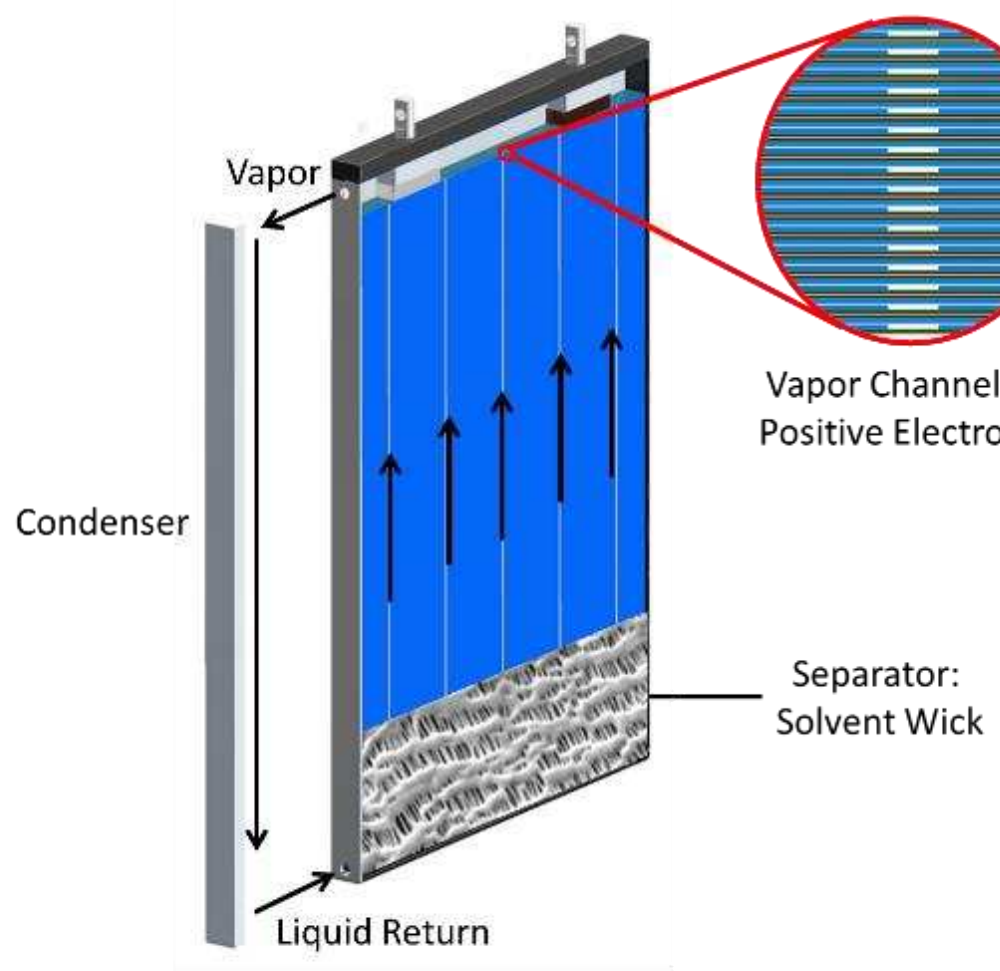

Section View

Figure 3-2: Proposed Internal Thermal Management System for Lithium-Ion Battery Using Volatile Co-Solvent in Electrolyte

The internal TMS concept is also proposed for an 18650 cell architecture (Figure 3-3). For this architecture, the jelly roll is held in the center of the cell can with plastic jelly roll supports. The uniform separation created between the jelly roll and the 18650 steel provides an internal annular condenser for vapor generated within the channels of the positive electrode. External convection cooling is applied to the exterior casing of the 18650 steel can to provide the condensing power required.

In both of the proposed architectures, vapor channels are created in the positive electrode of the cell. The

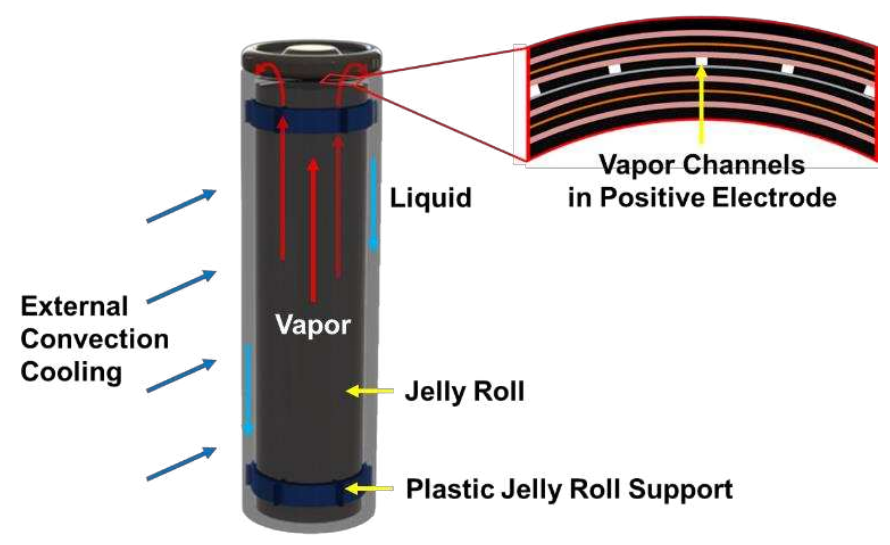
positive electrode is chosen due to the 
Removal of $<2 \%$ of Electrode Material: Generated Vapor Flow Path

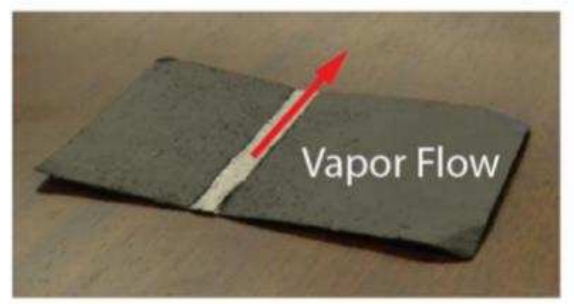

Micrograph of Vapor Channel

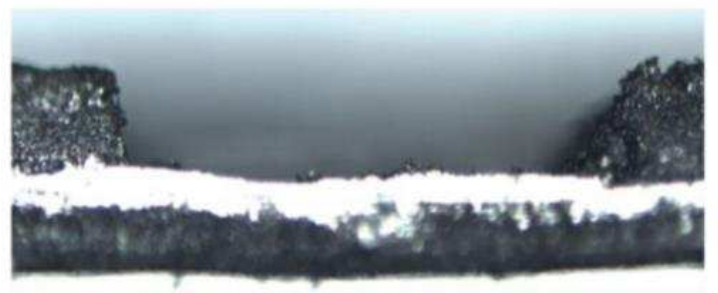

Figure 3-4: Representative Vapor Generation Channel Created in a Positive Electrode

possibility of lithium plating on exposed surfaces of copper. During charge, lithium concentrates in the negative electrode forcing its potential to decrease. In graphite electrodes, the potential in the negative electrode can approach $0 \mathrm{~V}$ vs. $\mathrm{Li} / \mathrm{Li}^{+}$, the potential at which the lithium plating reaction becomes active (see Section 1.2). Lithium preferentially plates in a dendritic fashion which can grow and create an electrical short to the cathode. For this reason, copper surfaces are never exposed to any electrochemically active surface area in commercial LIBs. Consequently, vapor generation channels can only be safely created in the positive electrode.

The cell modifications for the proposed internal TMS include the creation of evaporation channels and the integration of a condenser. The evaporator channels can be manufactured during the slurry coating process or completely removed after the electrode has been manufactured. In commercial cells, slurry-based positive electrode coatings uniformly cover the entire surface of the aluminum current collector and can range in thickness from 50-100 $\mu \mathrm{m}$. To create these evaporation channels during electrode manufacturing, the slurry is spread uniformly on the aluminum current collector everywhere except for masked portions designated for channels. Once the solvent used for slurry suspension has been evaporated and the electrode is calendared to its final thickness, the channel masking material can be removed without disturbing the surrounding coating. Alternatively, manufactured positive electrodes with a uniform active material coating can be modified to contain the channels. The active material coating can simply be abrasively 
removed from the aluminum current collector using a metal spatula or similar device. This type of channel manufacturing process is not a production-worthy approach, but it suffices for validation of the concept. In both of the proposed manufacturing methods, the channels extend the

entire vertical length of the electrode. Figure 3-4 shows a representative vapor generation channel that was created after the electrode material was uniformly coated on the current collector.

\section{$1 \mathrm{~mm}$}

Condenser 50:50 Waterl

Glycol
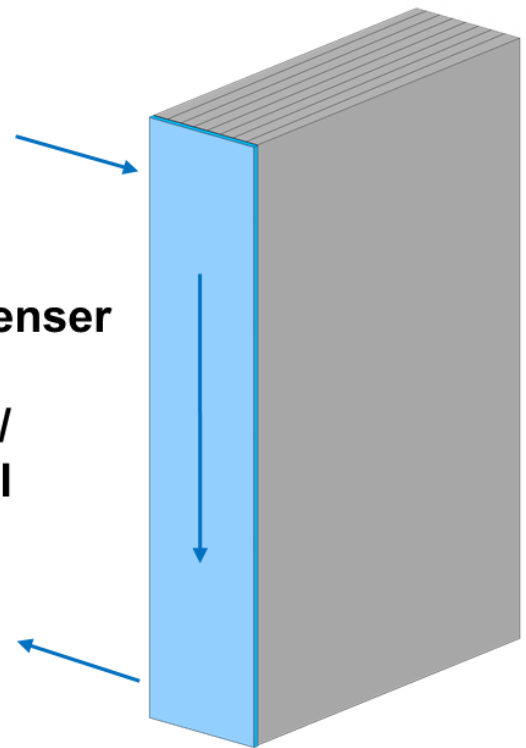

Figure 3-5: Embodiment 1 of Proposed Internal TMS with External Liquid Cooled Condenser on Edge Face of LIB Cells

The proposed internal TMS has two embodiments. In Figure 3-2, the condenser for the proposed internal TMS is shown integrated into the thin edge of the LIB cell. In this particular embodiment, a liquid coolant can flow through a structure in thermal contact with the condenser on the edge of the battery. Figure 3-5 shows a larger embodiment of the proposed internal TMS with a liquid cooled heat exchanger in contact with the thin edge of a group of cells. Water has an order of magnitude greater thermal conductivity and four times greater specific heat than air. This requires significantly less surface area for water to

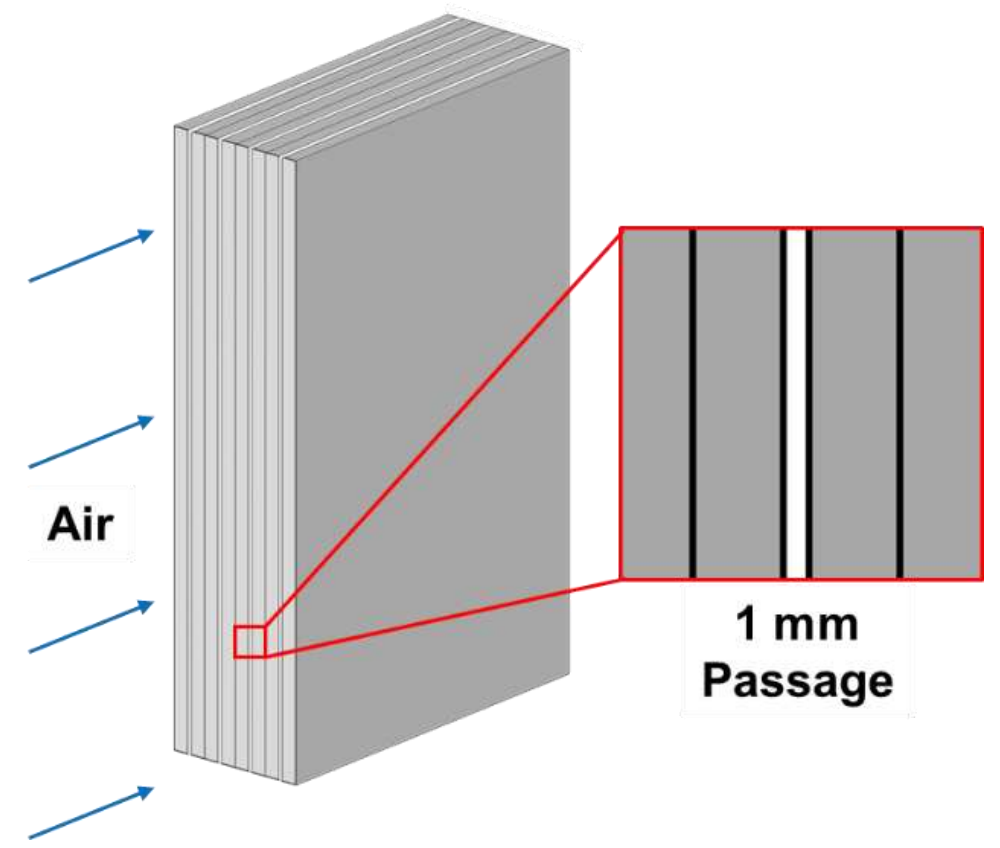

Figure 3-6: Embodiment 2 of Proposed Internal TMS with External Air Cooled Condenser on Large Face of LIB Cells 
reject the same about of heat as air. A second embodiment of the proposed internal TMS is shown in Figure 3-6. In this system, $1 \mathrm{~mm}$ spacing is created between every two cells to create a flow path for cooling air flow. This style of cell spacing is used in the Chevrolet Volt LIB pack; however, in the Volt, aluminum cooling plates occupy the $1 \mathrm{~mm}$ spacing. The system impact of the two proposed embodiments of the internal TMS is compared to the Chevrolet Volt in the next section.

\subsubsection{Impact of the Proposed Internal TMS}

To estimate the system impact of the proposed internal TMS, several assumptions were made. Table 3-1 lists the assumed density, dimensional, and quantitative values for the calculation. The analysis uses the dimensions of the 15 Ah Chevrolet Volt cell and the total system volume and mass are based upon the 288 cell architecture of the Volt LIB pack. In addition, the aluminum cooling plates used in the Chevrolet Volt TMS and the air passages created between cells in Figure 3-6 were assumed to occupy the same volume. The LIB cells in all three systems were also assumed to have identical performance of $240 \mathrm{Wh} \mathrm{kg}^{-1}$ and $640 \mathrm{Wh} \mathrm{L}^{-1}$. The LIB cells for the proposed internal TMS each contain 5 evaporation channels in every one of the positive electrodes

\section{Table 3-1: Values Used for System Impact of Proposed Internal TMS}

\begin{tabular}{|c|c|c|c|}
\hline Component & $\begin{array}{r}\text { Density } \\
\left(\mathrm{kg} \mathrm{L}^{-1}\right)\end{array}$ & & \\
\hline Air & 0.00123 & & \\
\hline 50:50 Water/Glycol & 1.05 & & \\
\hline Aluminum & 2.70 & & \\
\hline Positive Electrode Coating [30] & 1.30 & & \\
\hline Value & Cell & $\begin{array}{l}\text { Aluminum Plate/ } \\
\text { Air Channel }\end{array}$ & $\begin{array}{c}\text { Evaporation } \\
\text { Channel } \\
\end{array}$ \\
\hline Height $(\mathrm{cm})$ & 19.3 & 19.3 & 19.3 \\
\hline Width $(\mathrm{cm})$ & 14.5 & 14.5 & 0.1 \\
\hline Thickness (cm) & 0.5 & 0.1 & $0.0063 *$ \\
\hline Quantity in LIB Pack & 288 & 144 & 23,040 \\
\hline Quantity in Single Positive Electrode & - & - & 5 \\
\hline $\begin{array}{l}\text { Quantity of Positive Electrodes in Cell } \\
\text { Single-sided positive electrode coating thickness }\end{array}$ & - & - & 16 \\
\hline
\end{tabular}


within the cell (16 total). Each channel has dimensions of $19.3 \mathrm{~cm}$ long $\times 1 \mathrm{~mm}$ wide $\times 63 \mu \mathrm{m}$ deep. The depth of the channel is dictated by the positive electrode coating thickness, and all of the positive electrode coating is removed in the areas of the channel.

To quantify the impact on LIB performance from the creation of evaporation channels in the positive electrode of the $15 \mathrm{Ah}$ LIB cells, the mass of active material removed was calculated. The equivalent volume $(0.280 \mathrm{~L})$ of the 23,040 evaporation channels was calculated and the mass of active material removed was determined using the coating density $\left(1.30 \mathrm{~g} \mathrm{~L}^{-1}\right)$. The total electrode mass removed for 288 cells was determined to be $0.364 \mathrm{~kg}$. In addition, the total mass of the positive electrode active material (without channels) was calculated for the 288 cells and was determined to be $21.1 \mathrm{~kg}$. Therefore, the relative amount of positive electrode material removed for the modified cells is approximately $2 \%$. Assuming the cell is capacity limited by the positive electrode, removing the electrode material results in a proportional drop in the stored energy of the cell. As a result, the cell with evaporation channels have $87.8 \mathrm{Wh}$ of available energy, which is a $1.8 \mathrm{Wh}(2 \%)$ reduction.

The LIB pack + TMS volumetric and gravimetric energy density for each system was then calculated. This required the estimation of the total volume and mass of the cells and cooling infrastructure. The calculation did not consider the accompanying cooling manifold and distribution structure for any of the systems, and only the cooling plates and channels were considered. This results in a conservative estimate of the proposed MFE evaporation TMS for air cooling, but could underestimate the impact for liquid cooling. Equation (3.1) and (3.2) were used to determine the gravimetric and volumetric energy density of the LIB packs with a TMS:

$$
\text { Pack Gravimetric Capacity }=\frac{C_{\text {total }}}{m_{\mathrm{TMS}}+m_{\text {cells }}}
$$




$$
\text { Pack Volumetric Capacity }=\frac{C_{\text {total }}}{V_{\text {TMS }}+V_{\text {cells }}}
$$

where $C_{\text {total }}$ is the total stored energy capacity of the 288 cells, $m_{\text {TMS }}$ is the total mass of the external cooling infrastructure, $m_{\text {cells }}$ is the mass of the 288 cells, $V_{\text {TMS }}$ is the total volume of the external cooling infrastructure, and $V_{\text {cells }}$ is the total volume of the 288 cells. The results of the system comparison are shown in Table 3-2. Embodiments 1 and 2 of the proposed TMS improves the gravimetric capacity of the pack by $4.5 \%$ and $5.0 \%$, respectively, compared to the Chevrolet Volt. Embodiment 1 also offers a $6.6 \%$ increase in pack volumetric capacity as the $1 \mathrm{~mm}$ spacing between every two cells is removed and only a single aluminum cooling plate is added along the thin edge of the cells. Embodiment 2 requires that $1 \mathrm{~mm}$ spacing remain for the cooling air flow, and for the cell to be modified to contain evaporation channels; therefore, it slightly reduces the pack volumetric capacity compared to the Chevrolet Volt (2\%).

The impact of the proposed internal TMS is expected to be even greater when considering the entire cooling system architecture within the pack. The liquid manifold structure of the embodiment 1 is far simpler than that currently employed in the Chevrolet Volt, as only a single external cooling plate is required. Furthermore, the proposed internal TMS will maintain much more uniform cell temperatures as the distance for internally generated heat to be conducted is Table 3-2: System Impact of Proposed Internal TMS Compared to Chevrolet Volt Proposed Proposed

Calculated Quantity $\quad$ Embodiment 1 Embodiment 2 Chevrolet Volt

\begin{tabular}{lccc}
\hline Total Cell Volume (L) & 40.3 & 40.3 & 40.3 \\
\hline Total Cell Mass (kg) & 107 & 107 & 108 \\
\hline $\begin{array}{l}\text { External Cooling } \\
\text { System Volume (L) }\end{array}$ & 0.28 & 4.03 & 4.03 \\
\hline $\begin{array}{l}\text { External Cooling } \\
\text { System Mass (kg) }\end{array}$ & 0.52 & 0.005 & 7.56 \\
\hline $\begin{array}{l}\text { Pack Gravimetric } \\
\text { Capacity (Wh kg }\end{array}$ & 235 & 236 & 224 \\
\hline $\begin{array}{l}\text { Pack Volumetric } \\
\text { Capacity (Wh L }\end{array}$ & 623 & 570 & 582
\end{tabular}


Table 3-3: Summary of Current and Proposed TMSs for LIBs

\begin{tabular}{|c|c|c|c|c|c|}
\hline TMS & $\begin{array}{c}\text { External } \\
\text { Cooling } \\
\end{array}$ & $\begin{array}{c}\text { Internal } \\
\text { Cooling }\end{array}$ & $\begin{array}{c}\text { Currently } \\
\text { Implemented }\end{array}$ & $\begin{array}{c}\text { System } \\
\text { Complexity }\end{array}$ & $\begin{array}{c}\text { Effect of TMS } \\
\text { on LIB Pack } \\
\text { Energy Density }\end{array}$ \\
\hline Chevrolet Volt & Yes & No & Yes & High & Negative \\
\hline Tesla Model S & Yes & No & Yes & High & Negative \\
\hline $\begin{array}{l}\text { Bandhauer et al. } \\
\text { Microchannel } \\
\text { Cooling [72] }\end{array}$ & Yes & Yes & No & Medium & Negative \\
\hline $\begin{array}{l}\text { Mohammadian et } \\
\text { al. Single Phase } \\
\text { Electrolyte } \\
\text { Cooling [73] }\end{array}$ & Yes & Yes & No & High & Negative \\
\hline $\begin{array}{l}\text { Proposed Internal } \\
\text { TMS }\end{array}$ & Yes & Yes & No & Medium & Less Negative \\
\hline
\end{tabular}

minimized. A qualitative summary of the proposed internal TMS compared to other LIB TMSs is presented in Table 3-3.

Despite the benefits of the proposed internal TMS over conventional external TMS and other internal TMS concepts, fundamental questions remain to be resolved. First, a volatile cosolvent that is compatible with the lithium-ion chemistry and satisfies the thermal requirements of the TMS needs to be identified. Second, the electrochemical performance of a LIB undergoing continuous volatile co-solvent vapor generation needs be characterized. Third, the volatile cosolvent vapor generation has to be shown to have no detrimental effect on the LIB. The electrochemical and thermal requirements of the multi-functional electrolyte (MFE) for the proposed internal TMS are described in the next section.

\subsection{Multi-Functional Electrolyte Requirements}

To enable the proposed internal TMS, the MFE must satisfy several demanding electrochemical requirements of the LIB chemistry. In addition, the MFE must satisfy the thermal requirements that motivate this research effort. Both sets of requirements are presented in the following sections. 


\subsubsection{Electrochemical Requirements}

The electrochemical requirements of lithium-ion battery electrolytes are discussed in the reviews written by $\mathrm{Xu}[22,24]$, Aurbach et al. [94], and Jow et al. [95]. The list of electrochemical properties that must be satisfied by the electrolyte is extensive. As mentioned in Section 1.2.3, electrolytes can take many different forms and phases including liquid, polymer, solid, and ionic liquid. The focus of this discussion here is on liquid lithium-ion electrolytes. All liquid lithiumion electrolytes must be ionically conducting, electrically insulating, nonaqueous, aprotic, capable of solvating a high concentration of lithium salt, and have the ability to form a stable solid electrolyte interphase (SEI) on both the anode and cathode surfaces. Each of these are described in further detail in this section.

Ion conduction is the primary function of an electrolyte. High ionic conductivity enables the electrochemically active species (in this case, lithium-ions) to be readily available for oxidation and reduction at the surfaces of the active material particles of the anode and cathode. During cell operation, lithium ions are inserted into and de-inserted from the electrode materials. During deinsertion, the lithium ions are solvated in the electrolyte solution, and are transported to the other electrode surface via diffusion in the electrolyte. The rate of lithium ion transport is determined by the chemical potential gradient of the lithium ions in solution, which is proportional to the concentration gradient and the ionic conductivity. For electrolytes with poor ionic conductivity of lithium ions, a larger concentration gradient must develop within the electrolyte, which reduces cell performance by starving the insertion electrode of lithium ions. This effect reduces the cell potential when current is applied or withdrawn from the cell, and the difference between the open circuit potential and operation potential attributable to the effect is often deemed the concentration overpotential, which can also be expressed as a cell resistance. For high electronic conductivity 
electrodes, ionic conductivity can be the largest source of resistance in a cell [96]. The resistance of the cell is vital in determining its energy and power capability. Cells with a higher resistance (lower electrolyte ionic conductivity) have a more significant ohmic drop in voltage when current is drawn from them, reducing energy and power output. Liquid electrolytes have the highest ionic conductivity of all the different types because of the relative ease of lithium-ion transport in mixtures of lower viscosity. Not surprisingly, the majority of commercial lithium-ion cells utilize a liquid electrolyte. State-of-the-art liquid lithium-ion electrolytes have an ionic conductivity on the order of $5-10 \mathrm{mS} \mathrm{cm}^{-1}$ at room temperature [22].

Lithium-ion electrolytes must also be electrically insulating. The electrolyte and the separator exist between the anode and cathode interfaces of the cell (Figure 1-8). The separator is permeable to the liquid electrolyte, which allows for lithium-ion migration between the electrodes. The anode and cathode are both in direct contact with the separator, and, if the electrolyte is electrically conductive, the cell would immediately discharge and the potential difference would be 0 . A perfectly electrically insulating electrolyte will pass zero current over the entire operating potential range of the cell. In the event that either the separator or electrolyte fail to electrically insulate the anode and cathode from one another, an internal short will occur. These shorts vary in severity and can cause significant capacity fade or total cell failure. Severe internal shorts instantaneously release all the stored energy of the battery, which can cause violent thermal reactions to take place and trigger thermal runaway (see Section 1.3.1). This instantaneous energy

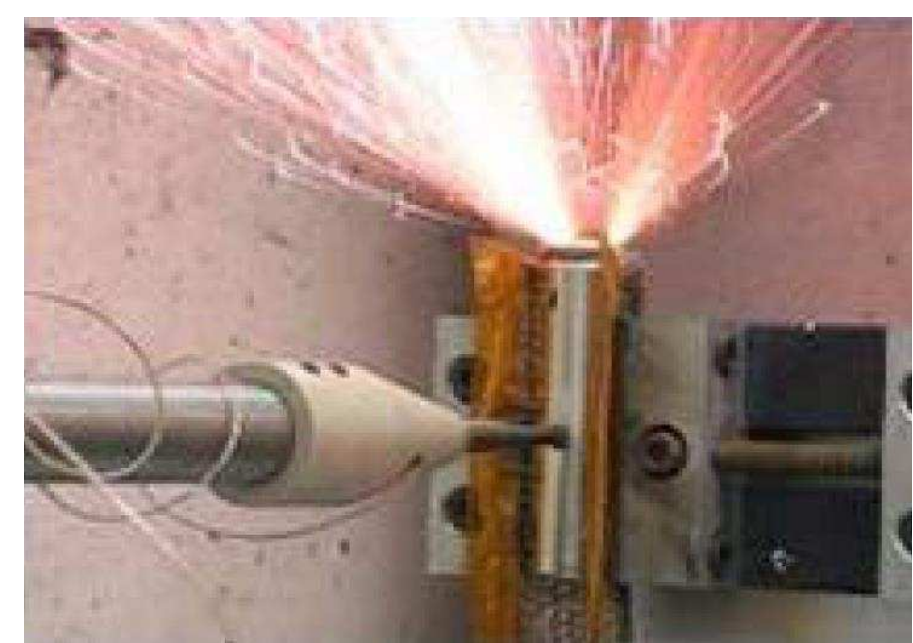

Figure 3-7: Internal Short Caused by Nail Penetration of a $\mathrm{LiCoO}_{2} / \mathrm{Graphite} \mathrm{Cell} \mathrm{at} 4.2 \mathrm{~V}$ [20] 
release is illustrated well in Figure 3-7 which shows a nail penetration test causing an internal electrical short in the cell. The nail penetration test is one of the most extreme tests of a LIB's thermal stability. Typically, the cell is fully charged into its most energetic state (the cell in Figure 3-7 was charged to $4.2 \mathrm{~V}$ ), and, when penetrated with a conductive rod, the stored energy in the battery is immediately dissipated via Ohmic heating. This rapid transfer of energy results in extreme heat generation, electrolyte volatilization, and ultimately fire.

The electrical stability of electrolytes is best understood by analyzing the liquid solvent molecules. It is widely accepted that the electrical stability of electrolytes is due the lowest unoccupied molecular orbital (LUMO) and the highest occupied molecular orbital (HOMO) of the solvent molecules [19]. The difference in energy states of the LUMO and HOMO of the electrolyte solvents provides a region of stable operation, $E_{g}$. There is not a sufficient driving potential to promote an electron from the HOMO or to accept an electron into the LUMO of the electrolyte solvents (Figure 3-8). The movement of electrons produces current, and would cause an internal short. Therefore, the LUMO and HOMO of the electrolyte solvents must be compatible with the

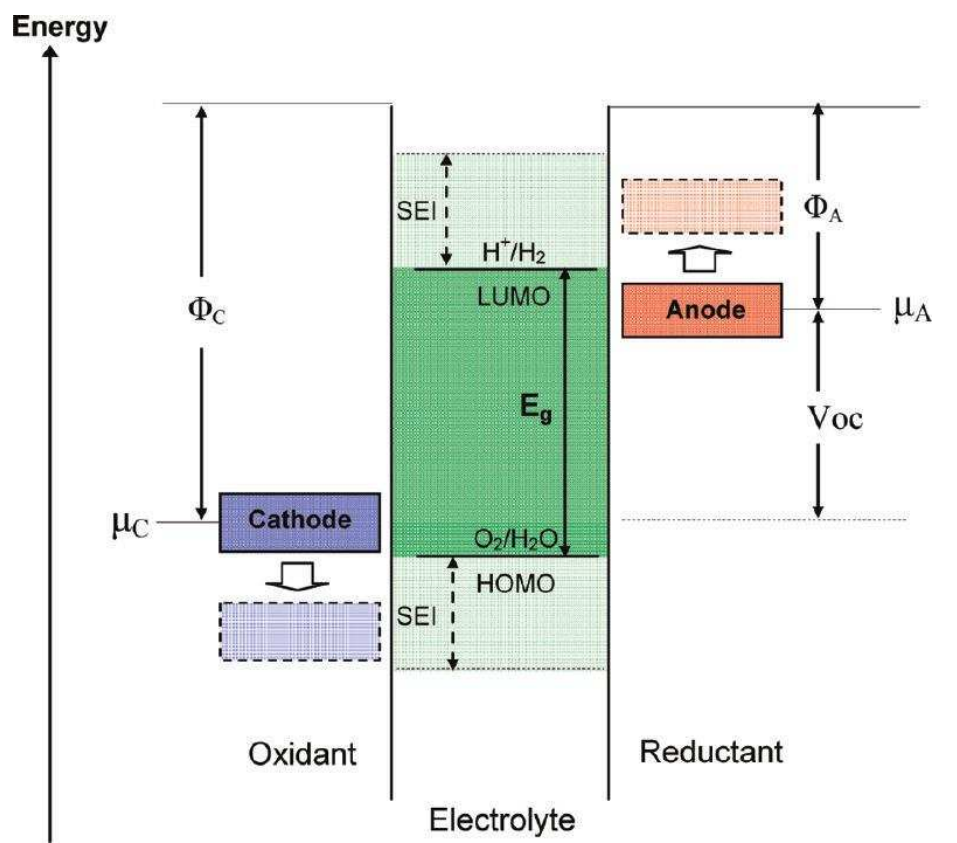

Figure 3-8: Open-Circuit Energy Diagram for a Lithium-Ion Electrolyte [19] potential window that lithium-ion chemistry operates, usually $0-5 \mathrm{~V}$ vs. $\mathrm{Li} / \mathrm{Li}^{+}[22]$.

The lithium-ion chemistry LUMO and HOMO constraints of the electrolyte solvents is the reason why nonaqueous electrolytes are required. Nonaqueous means that no water can be present in the electrolyte. In many 
lower voltage chemistries, water is an ideal solvent having abundant availability, a high dielectric constant to solvate a high concentration of salt, and a relatively low viscosity. However, water has two redox pathways that lie within the required operating potential window of a lithium-ion battery:

$$
\begin{gathered}
2 \mathrm{H}_{2} \mathrm{O}_{(\mathrm{l})}+2 e^{-} \rightleftharpoons \mathrm{H}_{2(\mathrm{~g})}+2 \mathrm{OH}^{-} \quad\left(2.21 \mathrm{~V} \mathrm{vs.} \mathrm{Li} / \mathrm{Li}^{+}\right) \\
2 H_{(\mathrm{aq})}^{+}+2 e^{-} \rightleftharpoons H_{2(\mathrm{~g})} \quad\left(3.04 \mathrm{~V} \text { vs. } \mathrm{Li} / \mathrm{Li}^{+}\right)
\end{gathered}
$$

Of greatest concern are the acidic protons of $\mathrm{H}^{+}$formed by the latter reaction, which readily react with components in the electrolyte such as $\mathrm{PF}_{6}{ }^{-}$anions to form $\mathrm{HF}$. $\mathrm{HF}$ is extremely corrosive and toxic and readily reacts to decompose the SEI layers of the anode and cathode. These reactions negatively affect the reversible cycling and storage life of cells [97]. Lithium-ion electrolytes are so adverse to water impurities that all electrolyte handing must be performed in an inert atmosphere that contain sub-ppm levels of water.

The nonaqueous requirement of lithium-ion electrolytes is further extended by the critical need for aprotic solvents. Protic solvents contain acidic protons of $\mathrm{H}^{+}$. This means solvent molecules that contain $\mathrm{O}-\mathrm{H}, \mathrm{N}-\mathrm{H}$, and $\mathrm{S}-\mathrm{H}$ bonds are unacceptable due to the ability for $\mathrm{H}^{+}$to dissociate from the molecule under the potentials seen in a lithium-ion battery. The O-H bond, present in alcohols and other molecules including water, has a dissociation energy of $87.8 \mathrm{kcal}$ $\mathrm{mol}^{-1}$ and is well-known to disable lithium-ion chemistry. The N-H bond is even weaker, having a dissociation energy of $72 \mathrm{kcal} \mathrm{mol}^{-1}$ [98]. The S-H bond is even weaker still, with a dissociation energy of $67 \mathrm{kcal} \mathrm{mol}^{-1}$ [99].

In addition, lithium-ion electrolyte solvents should not contain any halogens (Chlorine, Bromine, Iodine, Astatine). All commercially used liquid solvents are organic, meaning they contain carbon. The $\mathrm{C}-\mathrm{X}$ bond with a halogen is susceptible to dissociation under the potentials of 
lithium-ion chemistry and readily react with oppositely-charged lithium-ions in the electrolyte to form insoluble lithium salts. This reduces the availability of the lithium-ions for the powerproducing redox reactions. The C-F bond, however, is an exception to this requirement. Fluorine is the most electronegative element known to exist and it forms the strongest bond in organic chemistry when bonded to carbon [25]. Therefore, C-F bonds in solvent molecules are acceptable for lithium-ion chemistry.

All liquid lithium-ion electrolytes must also be capable of solvating a high concentration of lithium salt. As discussed in Section 1.2.3, lithium salts are the source of lithium-ions for cell operation. A solvent's ability to solvate a lithium salt is measurable by its dielectric constant. For purposes of understanding lithium-ion electrolyte chemistry, dielectric constant refers to solvent's ability to reorient itself in the presence of a charged molecule (i.e., lithium-ions). Solvation of a lithium salt occurs by the formation of a coordination sphere of solvent molecules around the lithium-ion. The coordination sphere neutralizes the charge of the cation (lithium-ion) and prevents attraction with its oppositely charged anion, which varies with every lithium salt. Figure 3-9 shows the coordination sphere of EC molecules around a lithium-ion in a $1.0 \mathrm{M} \mathrm{LiPF}_{6}$ solution. Ethylene carbonate has a high dielectric constant $(90.5$ [89]) which enables it to solvate the inorganic $\mathrm{LiPF}_{6}$ salt to high concentration.

Ideal electrolytes contain low viscosity solvents with high dielectric constants. The most common high dielectric constant solvent in lithium-ion chemistry is ethylene carbonate, which readily solvates $\mathrm{LiPF}_{6}$. The high dielectric

Figure 3-9: Ethylene Carbonate Coordination Sphere around a Positively Charge Lithium-Ion (Oxygen-red; carbon-green; lithium-blue) [22] 
constant of ethylene carbonate arises from the electronegativity of the carbonate ester group of the molecule which preferentially orients itself to positive charges [100]. Despite its high dielectric constant and ability to solvate inorganic lithium salts, ethylene carbonate cannot serve as the sole electrolyte solvent because it is a solid at room temperature. It must be mixed with lower viscosity linear organic solvents to form an ionically conductive mixture that has an acceptable viscosity for operation in a wide temperature range. These solvents, namely dimethyl carbonate (DMC), diethyl carbonate (DEC), and ethyl methyl carbonate (EMC), have much lower dielectric constants (2.83.1 [89]) and would not be capable of solvating the high concentration of the inorganic lithium salt on their own. The solution to mix linear and cyclic carbonate solvents for their favorable solvation and viscosity properties holds true for all conventional electrolyte mixtures.

Although used in commercial LIBs, high dielectric constant solvents are not necessarily required for lithium-ion electrolytes. Lithium salts are either organic or inorganic. Organic salts are more easily dissociated due to their larger anion size, which spreads its negative charge over the entire anion. As a result, a solvent does not need strong coordination spheres to keep the lithium-ions from becoming attracted to their bonding location on the anions in organic salts, and low dielectric constant linear organic carbonates are capable of solvating organic lithium salts to high concentration (> $1 \mathrm{M})$. Regardless of the lithium salt type, an ionic conductivity maximum typically occurs around a $1 \mathrm{M}$ concentration of the salt. Examples of this phenomena can be found in the work performed by Arai on using the organic salts LiBETI and LiTFSI [85], and in papers using various inorganic salts including $\mathrm{LiAsF}_{6}$ [101] and $\mathrm{LiPF}_{6}$ [23]. In summary, high dielectric constant solvents are required for solvation of a high concentration of inorganic lithium salts, while lower dielectric constant solvents are capable of solvating a high concentration of organic lithium salts, and both approaches form viable lithium-ion electrolytes. 
The final electrochemical requirement for lithium-ion electrolytes is the ability to form a stable SEI layer on the anode and cathode of the cell. In commercial cells, the operating potential of the battery often exceeds the LUMO and HOMO of the electrolyte solvents. The shortcoming of the electrical properties of the electrolyte can be overcome by the formation of a passivating layer on the active material of the anode and cathode. Figure 3-8 shows how a properly formed SEI layer on the anode and cathode can extend the reversible energy barrier of the electrolyte. This passivating layer consists of insoluble decomposed solvent molecules, salt anions, and lithium ions. The SEI formation process causes irreversible capacity loss of the cell due to consumption of the electrochemically active species, but, if it is properly formed, it will sustain reversible cycling operation for the life of the cell. A stable SEI will provide electrical insulation between the electrolyte and the active material to prevent further decomposition, and allow for lithium-ions to freely migrate through to the active material surface of the electrodes. The nature of the SEI varies for every possible combination of active material and electrolyte mixture, and is heavily influenced by the solvents in the electrolyte [24]. A stable SEI cannot be predicted for a new cell chemistry: it must be measured and the electrolyte mixture must be refined until a stable SEI is created. Verma et al. provides much more information on the formation of the SEI in LIBs, particularly on graphite [102].

In summary, the primary electrochemical requirements for the MFE are to be ionically conducting, electrically insulating, nonaqueous, aprotic, capable of solvating a high concentration of lithium salt, and capable of forming stable SEI passivation layers on the anode and cathode surfaces of the active materials. In the next section, the thermal requirements for a MFE in the proposed system are discussed in detail. 


\subsubsection{Thermal Requirements}

When compared to conventional electrolyte mixtures, the primary thermal requirements for the liquid multi-functional electrolyte (MFE) are completely counter-intuitive. Current research efforts have sought to formulate low vapor pressure electrolytes primarily to improve safety. This is because the majority of solvents are flammable, and vaporizing them could increase the likelihood of explosion if sufficient oxygen is present. In particular, the common solvents used to mitigate the high viscosity of ethylene carbonate, namely the linear organic carbonates DMC, DEC, and EMC, have dangerously low flash points $\left(18-25^{\circ} \mathrm{C}\right)$ [46]. Consequently, all electrolyte mixtures that contain these linear carbonates are extremely flammable, providing significant fuel during a thermal runaway failure. Because combustion takes place in the vapor phase, the minimization of electrolyte vapor pressure generally equates to greater thermal stability. This logic motivates the research for electrolytes based on ionic liquids which have a near zero vapor pressure [95]. In contrast, the proposed TMS requires the MFE to contain a solvent that has a high vapor pressure (i.e., low boiling point). In addition, because it is desired to minimize the amount of vapor generation, the co-solvent should also have a high enthalpy of vaporization.

Liquids that have a high vapor pressure at room temperature generally have a low boiling point at one atmosphere of pressure. Selecting a fluid with a boiling point $<40^{\circ} \mathrm{C}$ is critical for enabling the internal TMS to function well below temperatures that triggers thermal runaway. The boiling point establishes the temperature at which the cell will isothermally reject internally generated heat. If the MFE fails to boil before it reaches the temperatures associated with capacity degradation and thermal runaway, the proposed system is not viable. Carbonate co-solvents currently used in LIB electrolytes have an unacceptably high boiling point at 1 atm for the proposed 
system: $90.5^{\circ} \mathrm{C}$ for DMC, $107.5^{\circ} \mathrm{C}$ for EMC, and $126.8^{\circ} \mathrm{C}$ for DEC. Additional thermal properties of common carbonate co-solvents are provided in Section 3.3.2.

In addition, the solvent must have a high enthalpy of vaporization. The enthalpy of vaporization is a measure of the amount of energy per unit mass of fluid required during the liquidvapor phase change. The enthalpy of vaporization is a function of the fluid pressure. As the pressure of the fluid increases, the enthalpy of vaporization decreases. On a pressure-enthalpy diagram, the enthalpy of vaporization is defined as the difference in enthalpy of the fluid in the gaseous phase (100\% vapor quality) and the liquid phase (0\% vapor quality) at a set temperature and pressure. The vapor quality is the mass fraction of vapor in the two-phase liquid-vapor mixture. The saturated liquid and vapor enthalpies are illustrated in the P-h diagram for the fluid HFE-7000 (Figure 3-10). The red drop line is the fluid enthalpy at $100 \%$ vapor quality $\left(218 \mathrm{~kJ} \mathrm{~kg}^{-1}\right)$ and the blue line is the fluid enthalpy at $0 \%$ vapor quality $\left(85.22 \mathrm{~kJ} \mathrm{~kg}^{-1}\right)$. The difference of these two enthalpy values, $132.7 \mathrm{~kJ} \mathrm{~kg}^{-1}$, is the enthalpy of vaporization at $97 \mathrm{kPa}$ and $34^{\circ} \mathrm{C}$. Unfortunately, all liquid solvents that are feasible solvents for lithium-ion electrolytes have an order of magnitude lower enthalpy of vaporization than water, which is $2257 \mathrm{~kJ} \mathrm{~kg}^{-1}$ at $101 \mathrm{kPa}$ and $100^{\circ} \mathrm{C}$. Water has an unusually high enthalpy of vaporization due to strong hydrogen bonding between the relatively small water molecules in the liquid phase. These intermolecular forces must be overcome for evaporation to occur,

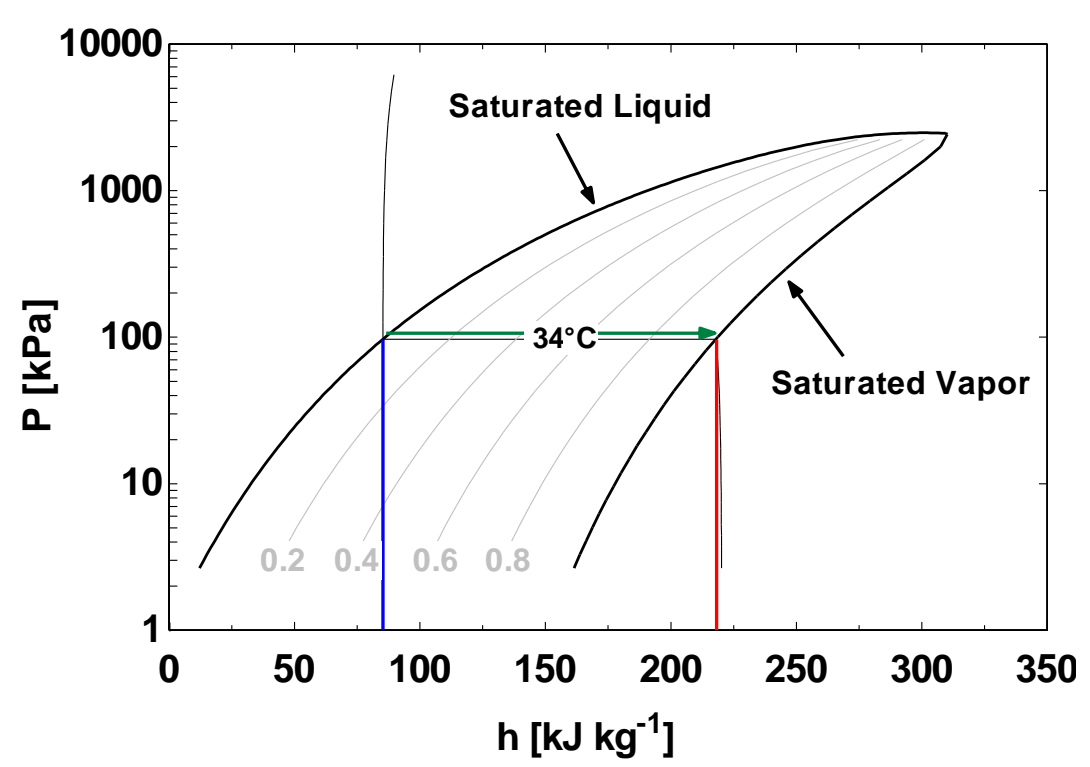

Figure 3-10: P-h Diagram of HFE-7000 with a $34^{\circ} \mathrm{C}$ Isotherm 
requiring a significant amount of energy. These phenomena are well described and quantified in water modeling efforts [103]. A high enthalpy of vaporization is desired to maximize the amount of two-phase heat transfer per unit mass of volatile co-solvent evaporated. In addition, a fluid with a high enthalpy of vaporization will require a lower evaporation rate to achieve the same rate of two-phase heat transfer as a fluid with a lower enthalpy of vaporization. In this scenario, more of the volatile co-solvent will remain a liquid while still rejecting the same amount of heat. Therefore, an ideal volatile electrolyte solvent will have a high enthalpy of vaporization, similar to that of water.

In summary, the two primary thermal requirements for evaporating co-solvent in a MFE are high vapor pressure and enthalpy of vaporization. In the next section, several candidate solvents that meet both the electrochemical and thermal requirements are discussed in detail.

\subsection{Components of the Multi-Functional Electrolyte}

The components of the MFE have a significant list of electrochemical and thermal requirements that must be met. Conventional electrolyte mixtures satisfy the demanding electrochemical requirements, as they have been successfully used in lithium-ion chemistry since the first commercial cell was produced in 1991. However, these conventional mixtures do not meet the thermal requirements of the proposed TMS. An exhaustive search was performed to identify candidate volatile solvents that satisfied all of the electrochemical and thermal requirements. The search led to two conclusions: (1) volatile solvents that meet the thermal and many of the electrochemical requirements exist and (2) these volatile solvents cannot serve as the only solvent in the electrolyte. Conclusion (2) is also the case for conventional electrolyte mixtures: multiple solvents are used to balance the benefits and shortcomings of each. For example, a $1 \mathrm{M} \mathrm{LiPF}_{6}$ electrolyte in $100 \% \mathrm{EC}$ at $20^{\circ} \mathrm{C}$ has an ionic conductivity of $6.9 \mathrm{mS} \mathrm{cm}^{-1}$; if $\mathrm{EC}$ is mixed $1: 1$ by 
weight with EMC, the conductivity increases to $8.5 \mathrm{mS} \mathrm{cm}^{-1}$ primarily due to the lower viscosity of EMC [1]. The term, co-solvent, will be used for the remainder of this document for the sole purpose of recognizing a single solvent's inability to satisfy all of the thermal and electrochemical requirements of the MFE.

In the following sections, the candidate volatile co-solvents are presented. Then, the candidate organic carbonate co-solvents that compensate for the electrochemical shortcomings of the volatile co-solvents are reviewed. After, the lithium salt suitable for the MFE is discussed. Finally, the baseline, conventional electrolyte mixture used for performance comparison during experimentation is identified.

\subsubsection{Candidate Volatile Co-Solvents}

After an extensive search, fluorinated organic fluids appear to be the best candidates for co-solvents in the MFE. The fluids are primarily perfluoroalkanes (Table 3-4), which are molecules with a carbon backbone saturated with fluorine atoms. Due to the plethora of strong CF bonds, these fluids have extreme chemical and thermal stability. Fluorine is the most electronegative element on the periodic table, which means that it preferentially attracts and retains electron bonding pairs. These bonding pairs are critical to determining the dielectric constant of the fluid, and create a high electron density around the fluorine atom of the C-F bond, which shortens the bond length between the carbon and fluorine [25]. Because the electrons are held close to the nucleus of the fluorine atom, the polarizability is lessened. Polarizability is the capacity of the molecule to reorient in the presence of an opposite charge. No lone electron pairs are available to attract other molecules, and the dielectric constant of the perfluoroalkanes is significantly reduced as a result. This is especially evident in the FC-72 and Perflenapent fluids. 


\begin{tabular}{|c|c|c|c|c|c|c|}
\hline $\begin{array}{l}\text { Candidate } \\
\text { Co-Solvent }\end{array}$ & $\begin{array}{c}\text { Boiling } \\
\text { Point at } \\
1 \text { atm } \\
\left({ }^{\circ} \mathbf{C}\right)\end{array}$ & $\begin{array}{c}\text { Vapor } \\
\text { Pressure } \\
\text { at } 25^{\circ} \mathrm{C} \\
(\mathbf{a t m})\end{array}$ & $\begin{array}{c}\text { Absolute } \\
\text { Viscosity } \\
\text { at } 25^{\circ} \mathrm{C} \\
\text { (cP) }\end{array}$ & $\begin{array}{l}\text { Dielectric } \\
\text { Constant }\end{array}$ & $\begin{array}{l}\text { Chemical } \\
\text { Formula } \\
\end{array}$ & Molecular Structure \\
\hline HFE 7000 & 34 & 0.638 & 0.45 & 7.4 & $\mathrm{C}_{4} \mathrm{H}_{3} \mathrm{~F}_{7} \mathrm{O}$ & \\
\hline HFE 7100 & 60 & 0.609 & 0.58 & 7.4 & $\mathrm{C}_{5} \mathrm{H}_{3} \mathrm{~F}_{9} \mathrm{O}$ & \\
\hline FC-72 & 56 & 0.296 & 0.64 & 1.8 & $\mathrm{C}_{6} \mathrm{~F}_{14}$ & \\
\hline Perflenapent & 29.2 & 0.857 & 0.472 & 1.8 & $\mathrm{C}_{5} \mathrm{~F}_{12}$ & \\
\hline
\end{tabular}

Table 3-4 shows four identified candidate co-solvents. All of these fluids have viable commercial sources, something that many other identified and unlisted candidate co-solvents did not. Two fluids, HFE-7000 and Perflenapent, are ideal candidates that satisfy the thermal requirements. These two fluids have favorably low boiling points and high vapor pressure. The other two, HFE-7100 and FC72, do not have boiling points that are less than $40^{\circ} \mathrm{C}$ at 1 atmosphere of pressure. However, if the pressure of the system is reduced, the fluids will be capable of boiling at lower temperatures. This effect is illustrated on

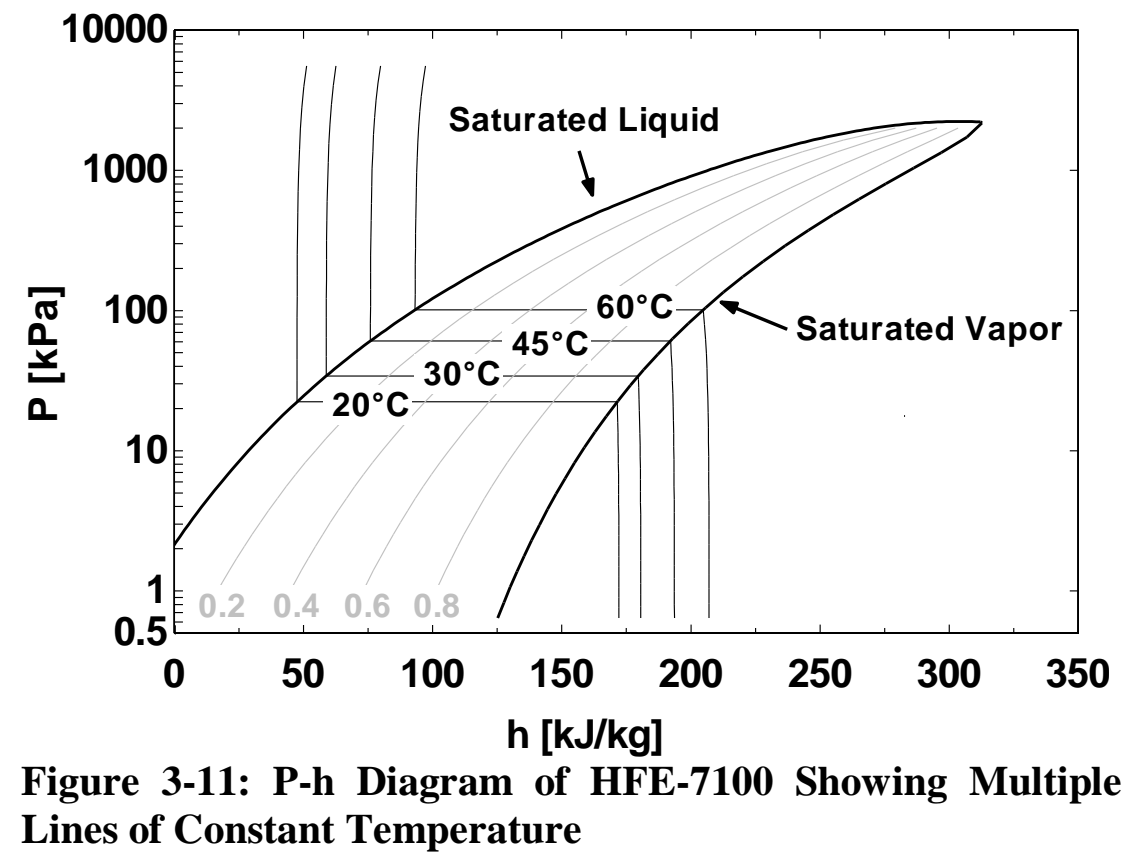


the P-h diagram of HFE-7100 (Figure 3-11). There are four isotherms on the diagram, two of which show the necessary reduction of vapor pressure to boil within a range of acceptable temperatures: $30^{\circ} \mathrm{C}$ and $45^{\circ} \mathrm{C} .30^{\circ} \mathrm{C}$ is the saturation (boiling) temperature of HFE-7100 at $34.2 \mathrm{kPa}$ of pressure (approximately 0.34 atmospheres). The saturation pressure at which HFE-7100 exists in the liquidvapor phase at $45^{\circ} \mathrm{C}$ is $60.7 \mathrm{kPa}$ of pressure (approximately 0.60 atmospheres). The reduction of system pressure can allow for a significant reduction in the boiling temperature of the fluid. Therefore, HFE-7100 and FC-72 are included in the candidate co-solvents list due to the ability to depress the boiling temperature to an acceptable value.

There are small, but significant, differences in the molecular structure between the HFE fluids and FC-72 and Perflenapent (Table 3-4). The fluorocarbon structure of the HFE fluids is interrupted by the addition of a methoxy group, an oxygen-methane $\left(\mathrm{O}-\mathrm{CH}_{3}\right)$ group, at one end of the molecule. The addition of this group dramatically affects the dielectric properties of the HFE fluids. For example, the dielectric constant of the HFE fluids is over four times greater than FC72 and Perflenapent. The methoxy group gives the HFE fluid molecules asymmetry, which creates a moment arm for the electronegative oxygen atom of the methoxy group to reorient the molecule in the presence of a positive charge. The measurement of this moment arm is called the dipole moment. Dipole moments can be calculated using complicated molecular orbital calculations that rely on ionization potential, electron affinity, and atomic radius [104]. A much more simple approach is to look for asymmetry in the molecular structure. Ideally, the asymmetry is combined with an electronegative atom, like oxygen. Lithium-ion electrolytes must be aprotic, so all dipole moments must be due to electronegative elements that rely on electrons, not protons, for their polarity. 
Due to the varying dielectric constants of the volatile co-solvents, differences in the lithium salt solvation capability of each fluid are expected. Based on their asymmetric molecular structure and consequently higher dielectric constant, HFE-7000 and HFE-7100 are expected to play a larger role in the solvation of the lithium salt. In contrast, FC-72 and Perflenapent are not expected to play a significant role in solvation. Using FC-72 and Perflenapent in a lithium-ion electrolyte hinges on their ability to be miscible with another co-solvent capable of lithium salt solvation and while not disrupting the solvation of the salt. The candidate organic carbonate co-solvents and lithium salt that were mixed with these candidate volatile co-solvents are now presented.

\subsubsection{Candidate Organic Carbonate Co-Solvents and Lithium Salt}

Conventional electrolyte mixtures utilize at least one linear carbonate solvent (DMC, EMC, or DEC) and at least one cyclic carbonate solvent (typically EC). Cyclic carbonate solvents have a high dielectric constant which is useful for solvating inorganic lithium salts. However, EC is a solid at room temperature, and a linear carbonate solvent is also used to lower the viscosity of the electrolyte mixture and maintain EC in the liquid state. More notably, all of the common organic carbonate solvents currently used in LIB electrolytes have a boiling point that is much greater than the onset temperature of thermal runaway $\left(>65^{\circ} \mathrm{C}\right.$, see Section 1.3.1). Ding et al. measured the bubble point of a DEC/PC solvent mixture, which was in excess of $120^{\circ} \mathrm{C}$ regardless of the PC content due to $\mathrm{DEC}$ 's boiling point of $126.8^{\circ} \mathrm{C}$ [105]. Under vacuum, these co-solvents will have

much lower boiling points, but the flammability of the generated vapor is a significant safety concern and gas generation in the cell can make vacuum conditions extremely difficult to maintain. Therefore, these native solvents cannot be used as the volatile co-solvent for the proposed internal TMS since thermal runaway reactions would occur before any liquid-vapor phase change cooling 


\begin{tabular}{|c|c|c|c|c|c|c|}
\hline $\begin{array}{l}\text { Organic } \\
\text { Carbonate } \\
\text { Solvent }\end{array}$ & $\begin{array}{l}\text { Boiling } \\
\text { Point at } 1 \\
\text { atm }\left({ }^{\circ} \mathbf{C}\right)\end{array}$ & $\begin{array}{c}\text { Melting } \\
\text { Point } \\
\left({ }^{\circ} \mathbf{C}\right)\end{array}$ & 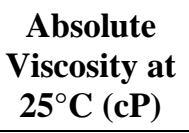 & $\begin{array}{l}\text { Dielectric } \\
\text { Constant }\end{array}$ & $\begin{array}{l}\text { Chemical } \\
\text { Formula }\end{array}$ & Molecular Structure \\
\hline $\begin{array}{l}\text { Dimethyl } \\
\text { Carbonate } \\
\text { (DMC) }\end{array}$ & 90.5 & 0.5 & 0.626 & 3.1 & $\mathrm{C}_{3} \mathrm{H}_{6} \mathrm{O}_{3}$ & 0 \\
\hline $\begin{array}{l}\text { Ethyl } \\
\text { Methyl } \\
\text { Carbonate } \\
\text { (EMC) }\end{array}$ & 107.5 & -53.8 & 0.690 & 2.9 & $\mathrm{C}_{4} \mathrm{H}_{8} \mathrm{O}_{3}$ & 0 \\
\hline $\begin{array}{l}\text { Diethyl } \\
\text { Carbonate } \\
\text { (DEC) }\end{array}$ & 126.8 & -43 & 0.75 & 2.8 & $\mathrm{C}_{5} \mathrm{H}_{10} \mathrm{O}_{3}$ & 0 \\
\hline $\begin{array}{l}\text { Propylene } \\
\text { Carbonate } \\
\text { (PC) }\end{array}$ & 241.7 & -48.8 & 2.5 & 64.4 & $\mathrm{C}_{4} \mathrm{H}_{6} \mathrm{O}_{3}$ & \\
\hline $\begin{array}{l}\text { Ethylene } \\
\text { Carbonate } \\
\text { (EC) }\end{array}$ & 248.2 & 36.4 & $1.86\left(40^{\circ} \mathrm{C}\right)$ & 90.5 & $\mathrm{C}_{3} \mathrm{H}_{4} \mathrm{O}_{3}$ & \\
\hline
\end{tabular}

could be realized. Table 3-5 shows basic thermal and chemical properties of common organic carbonate solvents used in commercial LIB liquid electrolytes.

Due to their similar viscosity and dielectric constant to the candidate volatile co-solvents, linear carbonate co-solvents are the most viable candidates to be miscible and provide lithium salt solvation to form the MFE. Arai exclusively used linear carbonate co-solvents in designing no flash point electrolytes with HFE-7100 and only later was able to add EC to the mixture in small amounts ( $0.5 \mathrm{M}$ concentration) $[85,86]$. Similarly, Naoi et al. found that linear carbonates were easily miscible with HFEs at any ratio, but ethylene carbonate, a cyclic carbonate, could only be added up to 5\% [90]. Notably, Arai found that EMC formed the highest conductivity mixture with HFE-7100 in comparison to DMC and DEC. Therefore, due to its superior performance with HFEs, EMC is selected as the candidate organic carbonate co-solvent for the MFE.

The selection of an electrolyte salt was limited solely to organic lithium salts due to the use of low dielectric constant co-solvents. Arai tested two organic lithium salts that produced high 
ionic conductivity solutions with HFE-7100 and EMC: LITFSI and LiBETI. LiTFSI is known to produce high ionic conductivity solutions compared to other organic lithium salts. Due to its plethora of strong C-F bonds in the anion, it also has high thermal stability. However, LiTFSI has been documented to corrode the aluminum current collector of the positive electrode at high potentials versus $\mathrm{Li} / \mathrm{Li}^{+}[106,107]$. LiBETI is a more fluorinated lithium salt than LiTFSI, has high thermal stability, and does not corrode the aluminum current collector. However, LiBETI has a lower ionic conductivity than LiTFSI $[85,95]$. Many other organic lithium salts exist including lithium triflate $\left(\mathrm{LiSO}_{3} \mathrm{CF}_{3}\right)$, lithium bis(oxalate)borate $\left(\mathrm{LiBOB}, \mathrm{LiB}\left(\mathrm{C}_{2} \mathrm{O}_{4}\right)_{2}\right)$, and lithium nonafluorobutylsulfonyl trifluoromethylsulfonyl imide (LiFBMSI, $\mathrm{LiN}\left(\mathrm{SO}_{2} \mathrm{C}_{4} \mathrm{~F}_{9}\right)\left(\mathrm{SO}_{2} \mathrm{CF}_{3}\right)$ ). Jow et al. provides much more information on all of these organic lithium salts [95]. Of all the organic lithium salts reported, only two had commercial sources at the time of this work, LiTFSI and LiBOB. Therefore, LiTFSI is chosen as the MFE lithium salt. As shown in Chapter 4, solvation of this salt to a $1 \mathrm{M}$ concentration by the candidate volatile co-solvents and EMC in a 1:1 by volume mixing ratio was attempted. Electrochemical tests with the MFE were compared to results from a baseline electrolyte, which is described in the next section.

\subsubsection{Baseline Electrolyte for Multi-Functional Electrolyte Performance Comparison}

With the introduction of novel volatile co-solvents into a lithium-ion electrolyte, it is necessary to compare the electrochemical performance of the MFE to that of a standard baseline. The most common linear carbonate co-solvents in commercial cells are DMC, DEC, and EMC. In this work, DEC is chosen as the linear carbonate co-solvent. The cyclic carbonate co-solvent will be ethylene carbonate due to is critical performance in forming a stable SEI [24]. In addition, this electrolyte must utilize lithium hexafluorophosphate $\left(\mathrm{LiPF}_{6}\right)$, also an industry standard for producing the highest ionic conductivity electrolytes. $\mathrm{LiPF}_{6}$ is known to form stable SEI 
passivation layers particularly on graphite anodes in lithium-ion cells [95]. For all non-boiling electrochemical experiments, the baseline electrolyte will consist of $1.0 \mathrm{M} \mathrm{LiPF}_{6}$ in 3:7 EC/DEC by weight percent. The baseline electrolyte was prepared by BASF.

\subsection{Summary}

In this chapter, a proposed internal TMS that utilizes a MFE was described. The operation principles and required LIB cell modifications were presented. The impact of the proposed system was investigated with battery pack energy density, system reliability, and system simplicity arguments compared to conventional, external TMSs. The electrochemical and thermal requirements for the MFE were discussed. The MFE must be ionically conducting, electrically insulating, nonaqueous, aprotic, capable of solvating a high concentration of lithium salt, and capable of forming stable SEI passivation layers on the anode and cathode surfaces of the active materials. The MFE must also contain a volatile co-solvent with a high vapor pressure, low boiling point, and high enthalpy of vaporization.

After reviewing the proposed TMS and the requirements of the MFE, the candidate components of the electrolyte were presented. Four fluids were presented as candidate volatile cosolvents; these fluids are perfluorocarbons or perflurocarbons with a methoxy group, and have sufficiently low boiling points to enable the operation of proposed internal TMS. Due to the miscibility limitations of cyclic carbonates with fluids similar to those of the candidate volatile cosolvents, linear carbonates are the only co-solvents initially considered as the MFE co-solvent. In particular, EMC is chosen as the linear carbonate co-solvent due to its proven miscibility with HFEs and high ionic conductivity in electrolyte solutions containing organic lithium salts. Because the MFE mixture does not contain high dielectric constant solvents, organic lithium salts are used in the present study for the MFE, and, due to its high solubility, ionic conductivity, and commercial 
availability, LiTFSI was chosen as the lithium salt for the MFE. The MFE will be 1.0 M LiTFSI in 1:1 candidate volatile co-solvent/EMC by volume, and its performance is compated to a baseline electrolyte (1.0 $\mathrm{M} \mathrm{LiPF}_{6}$ in 3:7 EC/DEC by weight percent). In the next chapter, the experimental procedures and results are discussed for the baseline electrolyte and a MFE that is held at a sufficiently low temperature to prevent boiling. 


\section{CHAPTER 4. NON-BOILING ELECTROCHEMICAL PERFORMANCE OF THE MULTI- FUNCTIONAL ELECTROLYTE}

With the exception of HFE-7100, none of the candidate volatile co-solvents have been investigated in a published research study for lithium-ion chemistry. The non-boiling experiments to characterize performance of the MFE with different co-solvents include miscibility, solubility, conductivity, electrochemical stability window, half cell cycling, full cell cycling, and impedance spectroscopy. Each of these experimental techniques is described in detail here, including relevant theory, experimental setup, data collection and processing procedures, and is followed by a discussion of the results.

The non-boiling electrochemical performance results presented in this chapter are critical for evaluating the proposed TMS. If the MFE fails to have comparable performance to the baseline electrolyte under non-boiling conditions, the proposed TMS will not provide any benefit to large lithium-ion battery packs. Instead, it will negatively compromise the fundamental purpose of the battery pack: to provide useful energy when it is required. The following sections will characterize the performance of the MFE in reference to the baseline.

\subsection{Miscibility \& Solubility}

Miscibility and solubility measurements are the first critical tests for creating a MFE. Each candidate volatile co-solvent must be miscible with EMC to form a homogeneous mixture. Nonhomogeneous mixtures of the co-solvents will result in an imbalance of lithium salt solvation between the two fluids and ultimately varying levels of conductivity through the electrolyte. A homogeneous mixture of co-solvents must also be capable of solvating a $1 \mathrm{M}$ concentration of LiTFSI salt to achieve the maximum possible ionic conductivity. If a homogenous mixture fails to solvate a $1 \mathrm{M}$ concentration of the LiTFSI salt, it is not feasible for the MFE. 
Table 4-1 provides the supplier list for the components of the MFE. In the following sections, the experimental procedures and equipment used for determining miscibility of the cosolvent with DMC is discussed first. The results from this experiment are then discussed.

Table 4-1: Suppliers for Components of Multi-Functional Electrolyte Multi-Functional Electrolyte Component

\begin{tabular}{llll} 
Description & CAS Number & Supplier & $\begin{array}{l}\text { Supplier Part } \\
\text { Number }\end{array}$ \\
$\begin{array}{l}\text { Volatile } \\
\text { Co-Solvent }\end{array}$ & $375-03-1$ & $3 \mathrm{M}$ & 98021229699 \\
$\begin{array}{l}\text { Volatile } \\
\text { Co-Solvent }\end{array}$ & $163702-07-6$ & $3 \mathrm{M}$ & 98021189406 \\
\hline $\begin{array}{l}\text { Volatile } \\
\text { Co-Solvent }\end{array}$ & $678-26-2$ & Exfluor & $\begin{array}{l}\text { C5 - } \\
\text { Perfluoropentane }\end{array}$ \\
$\begin{array}{l}\text { Volatile } \\
\text { Co-Solvent }\end{array}$ & $355-42-0$ & $3 \mathrm{M}$ & 98021102672 \\
$\begin{array}{l}\text { Carbonate } \\
\text { Co-Solvent }\end{array}$ & $623-53-0$ & $\begin{array}{l}\text { Sigma } \\
\text { Aldrich }\end{array}$ & 754935 \\
\hline $\begin{array}{l}\text { Lithium Salt } \\
\text { Sarigma }\end{array}$ & $90076-65-6$ & $\begin{array}{l}\text { Sigmich } \\
\text { Aldrich }\end{array}$ & 544094
\end{tabular}

\subsubsection{Method of Measurement}

Both miscibility and solubility measurements rely on visual inspection of the mixture to determine if any liquid separation or lithium salt precipitation occurs. If the candidate volatile cosolvent and EMC form a separated mixture, a MFE cannot be made and the volatile co-solvent is determined to be not feasible for the proposed TMS. All electrolyte mixtures are created in 1 fluid ounce $(30 \mathrm{~mL})$ glass jars to allow for direct viewing. The glass jars were purchased from Qorpak (part number GLA-00850). The small, wide mouth jars were critical for ease of electrolyte mixing and to interface with the conductivity sensor described in Section 4.2. The selection for jars was guided primarily by requiring the lowest electrolyte solution volume to provide conductivity measurements — all components of the electrolyte mixture were very expensive: $\$ 2.34 \mathrm{~mL}^{-1}$ for EMC, $\$ 3.95 \mathrm{~g}^{-1}$ for LiTFSI, and varying prices for the volatile co-solvents (from $\$ 0.85 \mathrm{~mL}^{-1}$ to 
$\left.\$ 1.61 \mathrm{~mL}^{-1}\right)$. In addition, the glass jars used caps with F217 and PTFE liners to create a chemicallyinert air-tight seal (Qorpak part number CAP-00065). The high quality seal the F217 and PTFE liners creates is critical for electrolyte mixtures containing high vapor pressure co-solvents which more readily evaporate than less volatile carbonate solvents.

\subsubsection{Test Parameters and Equipment Used}

Before mixing, all the candidate volatile co-solvents and EMC underwent the freeze-pump-thaw degassing procedure. The details of the procedure are located in Appendix A. The procedure requires the use of liquid nitrogen dewars, Schlenk flasks, a vacuum pump, and a heated stir plate. Upon successful completion of the

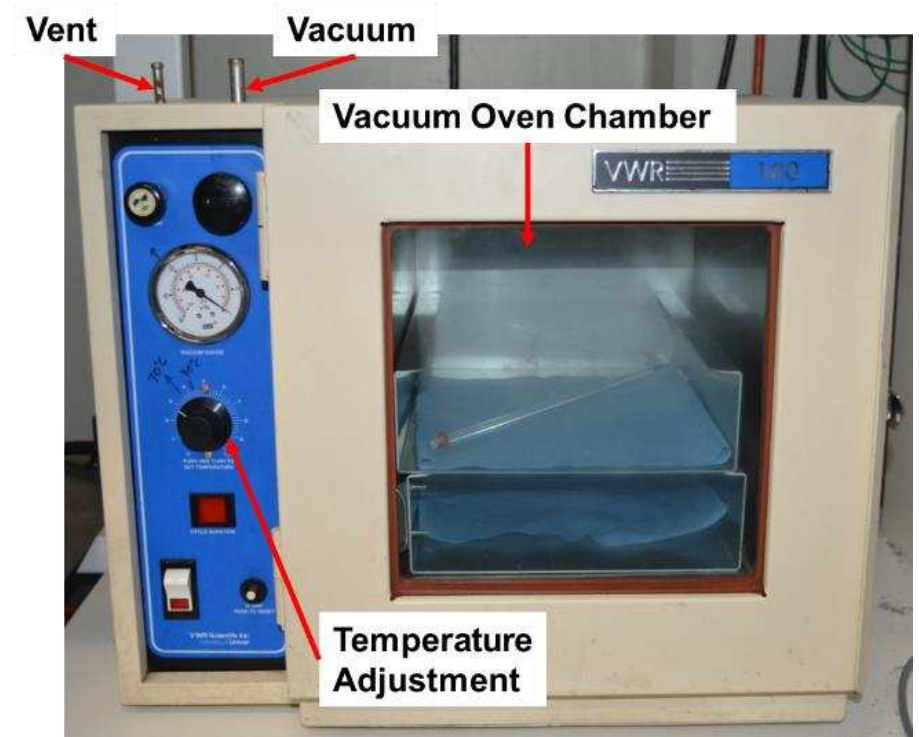

Figure 4-1: Vacuum Oven

procedure, all dissolved gasses (primarily $\mathrm{O}_{2}$ and $\mathrm{H}_{2} \mathrm{O}$ ) are removed from the fluids prior to pumping into an MBraun argon glove box $\left(\mathrm{O}_{2}\right.$ and $\mathrm{H}_{2} \mathrm{O}$ concentrations $<1$ ppm each). In addition, the glass jars and caps were cleaned with the following solvents in the specified order: acetone, methanol, isopropanol. The jars were then vacuum dried overnight at $80^{\circ} \mathrm{C}$ before being pumped into the argon glove box. Figure 4-1 shows the vacuum oven used for all component drying in this work.

Once inside the glove box, $12.5 \mathrm{~mL}$ of the candidate volatile co-solvent and EMC were drawn from their respective Schlenk flasks using a dedicated $6 \mathrm{~mL}$ polypropylene polyethylene (PP/PE) luer lock syringe with a 20 gauge needle. PP/PE has excellent material compatibility with 
lithium-ion electrolyte solvents (the same material is used for the separator material in LIBs). The $12.5 \mathrm{~mL}$ of the candidate volatile co-solvent and EMC were inserted into the $30 \mathrm{~mL}$ glass jar. The jar was promptly sealed to prevent loss of the volatile co-solvent. At this point, determination of co-solvent miscibility was made. If the co-solvents failed to be miscible, the separated mixture was documented with a picture, and no further testing was performed with that particular volatile co-solvent.

After successful completion of the miscibility test, a $1 \mathrm{M}$ concentration of LiTFSI salt was mixed in the co-solvent mixture. The molecular weight of LiTFSI salt is $287.09 \mathrm{~g} \mathrm{~mol}^{-1}$, which requires $7.177 \mathrm{~g}$ of the salt to be solvated in the $25 \mathrm{~mL}$ co-solvent mixture to have a $1 \mathrm{M}$ concentration. The mass of the LiTFSI salt was measured with a $7 \mathrm{~mL}$ weigh boat on a Mettler Toledo scale (MS104S/03) with a $0.1 \mathrm{mg}$ accuracy and $120 \mathrm{~g}$ maximum capacity. A clean, disposable polypropylene spatula was used to adjust the amount of LiTFSI salt in the weight boat. The LiTFSI salt was then placed into the glass jar containing the miscible co-solvents. A Teflon stirring bar $(3.18 \mathrm{~mm} \times 9.53 \mathrm{~mm})$ was also placed into the glass jar to aid in solvation; the glass jar was then promptly resealed. The MFE mixture was placed on a magnetic stirrer set at medium speed (IKA Topolino S1, $250 \mathrm{~mL}$ maximum stirring capacity). The mixture was allowed a minimum of 1 hour to complete the LiTFSI solvation process, indicated by a clear electrolyte solution (i.e., no remaining white LiTFSI particles). If complete LiTFSI solvation was observed, the candidate multi-functional electrolyte proceeded to the remainder of the electrochemical experiments.

\subsubsection{Results and Discussion}

Based upon the prior work of Arai, it was known that HFE-7100 was miscible with EMC and was capable of solvating a $1 \mathrm{M}$ concentration of LiTFSI salt. Therefore, initial efforts focused 
primarily on the other three candidate volatile co-solvents: HFE-7000, Perflenapent, and FC-72. Immediately upon mixing Perflenapent and EMC, it became evident that the two fluids were not miscible. In addition, FC-72 and EMC were also not miscible. The separated mixtures of Perflenapent/EMC and FC-72/EMC are seen in Figure 4-2. Both Perflenapent and FC-72 have higher liquid densities, $1.63 \mathrm{~g} \mathrm{~mL}^{-1}$ and $1.68 \mathrm{~g} \mathrm{~mL}^{-1}$ respectively, than EMC which has a density

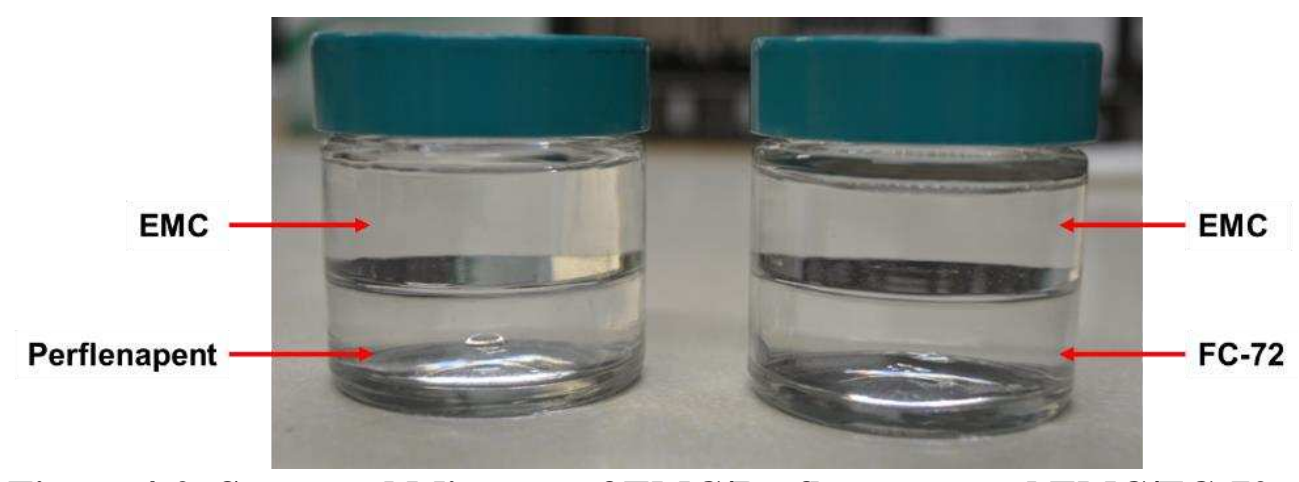

Figure 4-2: Separated Mixtures of EMC/Perflenapent and EMC/FC-72

of $1.006 \mathrm{~g} \mathrm{~mL}^{-1}$. Once combined, the two fluids settled beneath EMC under the influence of gravity. HFE-7000 was successfully mixed with EMC despite its high liquid density, $1.40 \mathrm{~g} \mathrm{~mL}^{-1}$. In addition, HFE-7100 with a liquid density of $1.51 \mathrm{~g} \mathrm{~mL}^{-1}$, was also successfully mixed with EMC, just as Arai had previously proven.

The success and failure of miscibility with EMC is a direct consequence of the difference in the molecular structure and polarity of the volatile co-solvents. Table 3-4 provides both the dielectric constant and molecular structure for each of the candidate volatile co-solvents. Both Perflenapent and FC-72 are symmetric perfluorocarbons, with no additional groups in the molecule. These highly fluorinated molecules have low dielectric constant (1.8) compared to the two HFEs which both feature a methoxy group at one end of the molecule (7.4). C-F bonds do have some polarity, but the high electronegativity of fluorine atom impairs polarity and the resultant dielectric constant of the molecule is low [25]. In addition, the symmetric molecular structure of Perflenapent and FC-72 cause any small dipole moment that might be created from 
the C-F bonds to be cancelled by an oppositely positioned C-F bond on the molecule. The result of these two phenomena prevent mixing with fluids that have higher polarities and dielectric constants. The asymmetry of the carbonate ester group of EMC gives the molecule polarity due to the lone electron pairs of the oxygen atoms. The methoxy group of HFE-7000 and HFE-7100 give the molecules polarity for the same reason as EMC. The similarity between the polarities of the HFE fluids and EMC is the primary reason for their miscibility.

Both the HFE-7000/EMC and the HFE-7100/EMC mixtures were capable of solvating a 1 M concentration of LiTFSI salt. Upon sufficient agitation provided by the stirring bar, both mixtures produced clear solutions with no indication of LiTFSI particles remaining unsolvated (Figure 4-3). Due to the much lower boiling point of $\mathrm{HFE}-7000,34^{\circ} \mathrm{C}$ at 1 atm, the HFE-7000/EMC mixture is explored for its performance in the remaining non-boiling and boiling

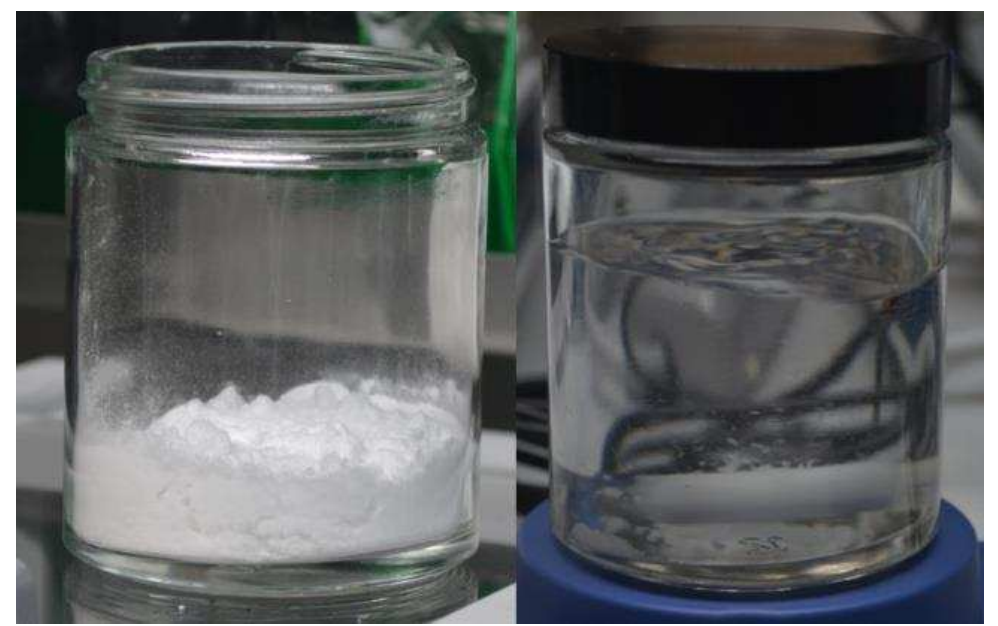

Figure 4-3: Unsolvated LiTFSI Salt (Left) and MFE Solution (1 M LiTFSI in 1:1 HFE-7000/EMC by Volume) on Stir Plate (Right) electrochemical experiments in this work.

\subsection{Ionic Conductivity}

As described in Section 3.2.1, the ionic conductivity of the electrolyte is measure of the availability of lithium-ions for redox reactions at the anode and cathode interfaces. It can also be a significant source of cell impedance: low ionic conductivity electrolytes cause cells to have reduced power performance. A description of the conductivity apparatus used and resulting measurements of the candidate multi-functional electrolyte is provided below. 


\subsubsection{Test Parameters and Equipment Used}

The conductivity testing is performed with a Rosemount Analytical 400 Endurance Conductivity Sensor with a cell constant of $1.0 \mathrm{~cm}^{-1}$. The cell constant maintains linearity within $\pm 0.6 \%$ of the reading for conductivity measurements in the range of $1 \mu \mathrm{S} \mathrm{cm}^{-1}$ to $20 \mathrm{mS} \mathrm{cm}^{-1}$. The sensor's electrodes are made of concentric pieces of titanium separated by a PEEK insulator. The sensor is also equipped with a Platinum 1000 resistance thermometer. Figure 4-4 shows the conductivity probe which contains $19 \mathrm{~mm}$ MNPT thread that was interfaced with a modified F217 and PTFE lined lid for the glass jar described in Section 4.1.1. The wall thickness of the lid was increased by curing an epoxy (Freeman Repro 83) on top of it. A $19 \mathrm{~mm}$ FNPT thread was cut into the modified lid so that it could accept and seal the conductivity probe. The modification of the jar lid to seal with the conductivity probe was critical for ensuring that minimal volatile co-solvent was lost during the measurement. The conductivity measurement of the probe is read and displayed by a 1056 Rosemount Analytical Dual Input Analyzer. The conductivity sensor and analyzer were factory loop calibrated in a $\mathrm{KCl}$ solution at the time of purchase. The reading displayed by the analyzer was recorded as the conductivity of the electrolyte. The equilibration time between

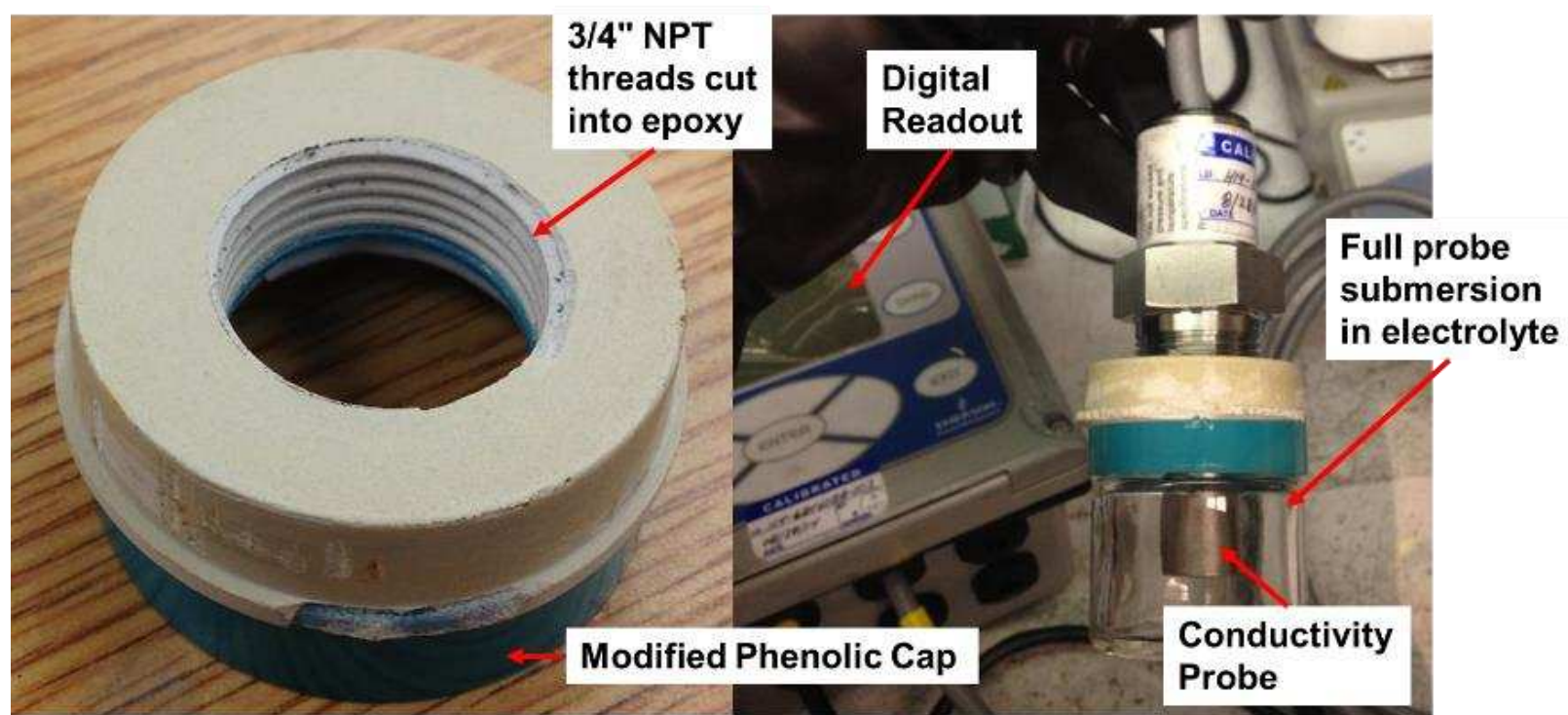

Figure 4-4: Modified Glass Jar Lid for Conductivity Probe (Left) and Conductivity Probe Submersion in Electrolyte (Right) 
submersion of the probe in the electrolyte, and a stable reading was typically achieved in less than one minute.

The ionic conductivity of the electrolyte is a strong function of temperature. This is a direct result of the change of electrolyte viscosity and dielectric constant with temperature, as shown by the thermodynamic analyses performed by Ding et al. on PC/DEC and PC/EC solvent mixtures [105]. High temperatures lead to greater lithium-ion mobility and higher conductivity due to the lower viscosity of the solvents. The opposite occurs as the temperature of the electrolyte is reduced: the viscosity of the solvent increases until the mixture begins to solidify. At this point, no lithiumion transport is possible and the conductivity plummets to zero [23]. The ionic conductivity measurements reported in this work were taken at room temperature (approximately $22^{\circ} \mathrm{C}$ ).

\subsubsection{Results and Discussion}

Table 4-2 shows the ionic conductivity of two candidate MFEs as well as the ionic conductivity of the carbonate-only baseline electrolyte. The results show the baseline electrolyte has the highest electrolyte conductivity followed by E1 (the HFE-7000 containing electrolyte) and E2 (the HFE-7100 containing electrolyte). These results were not unexpected: the use of the organic salt, LiTFSI, lowers the ionic conductivity of the electrolyte due to the reduced mobility of lithium-ions by the large anion size. In addition, the size of the volatile co-solvent molecule appears to have a measureable effect on the conductivity of the electrolyte solution. HFE-7100 contains one additional carbon atom bonded with two additional fluorine atoms compared to HFE-

\section{Table 4-2: Room Temperature Ionic Conductivity of Investigated Electrolytes}

\begin{tabular}{llc}
$\begin{array}{l}\text { Electrolyte } \\
\text { Name }\end{array}$ & Composition & $\begin{array}{c}\text { Room Temperature Ionic } \\
\text { Conductivity }\left(\mathbf{m S ~ c m}^{-1}\right)\end{array}$ \\
\hline E1 & $1.0 \mathrm{M} \mathrm{LiTFSI}$ in 1:1 HFE-7000/EMC by vol. & 2.309 \\
\hline E2 & $1.0 \mathrm{M} \mathrm{LiTFSI}$ in 1:1 HFE-7100/EMC by vol. & 1.463 \\
\hline Baseline & $1.0 \mathrm{M} \mathrm{LiPF}_{6}$ in 3:7 EC/DEC by wt. & 7.258
\end{tabular}


7000. The growth in molecular size of the co-solvent appears to negatively affect the ionic conductivity by reducing the $\mathrm{Li}^{+}$cation mobility.

The reduced ionic conductivity of the candidate MFEs is expected to result in a higher cell impedance compared to cells with the baseline electrolyte. Arai observed higher cell impedance using impedance spectroscopy for a $1 \mathrm{M} \mathrm{LiBETI}$ 8:2 HFE-7100/EMC electrolyte compared to a carbonate-only $1 \mathrm{M} \mathrm{LiPF}_{6}$ 3:7 EC/EMC electrolyte [86]. Despite having a lower ionic conductivity, the candidate MFEs need to be characterized in half and full cell cycling tests to truly realize the impact of the higher impedance resulting from the lower ionic conductivity. For the remainder of this work, only the E1 mixture is tested due to more favorable thermal properties of HFE-7000. MFE and E1 are used interchangeably to describe the same candidate electrolyte mixture.

\subsection{Electrochemical Stability Window}

The electrochemical stability window, or potential stability window, is a potential range that the electrolyte does not continually decompose via oxidation or reduction reactions. The potential range for an electrolyte is dictated by the LUMO and HOMO of the solvents used in the electrolyte (see Section 3.2.1). Naoi et al. provides the LUMO and HOMO for HFE-7100 and compared them to standard carbonate solvents (Figure 4-5) [90]. HFE-7100 has a greater HOMO than all of the standard

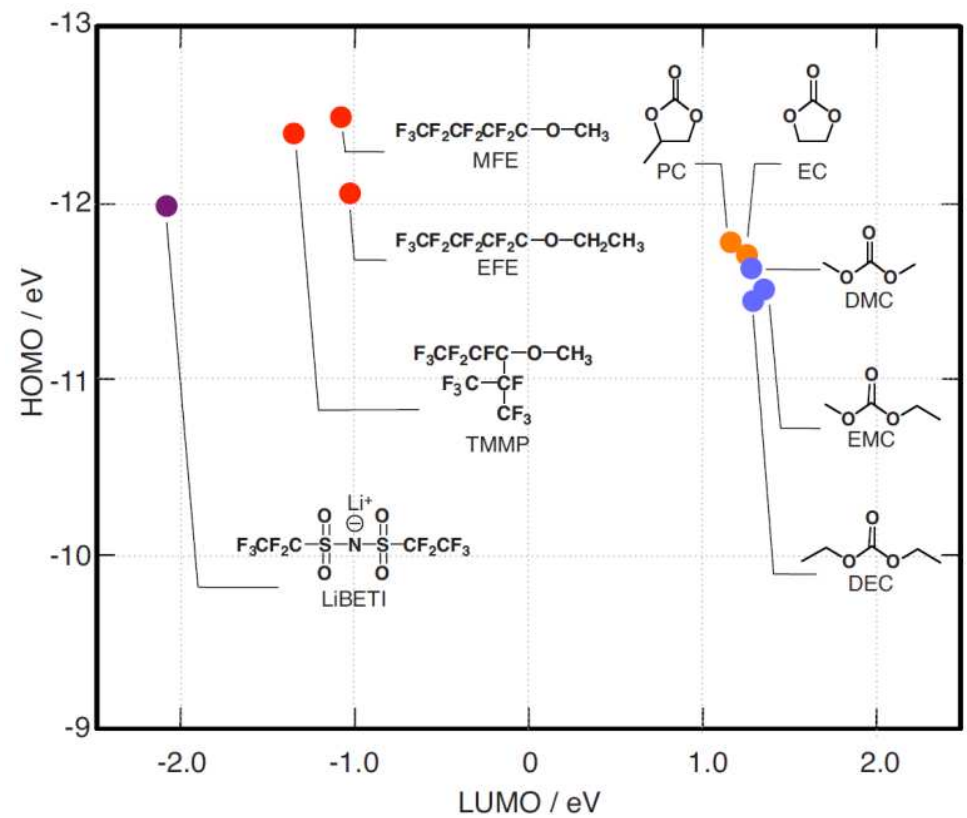

Figure 4-5: LUMO and HOMO of HFE-7100 (labeled as MFE) Compared to Other Carbonate Solvents [90] 
carbonate solvents, suggesting greater resistance against oxidation at high potentials versus $\mathrm{Li} / \mathrm{Li}^{+}$. However, the improved oxidative stability of HFE-7100 comes at a consequence to its reductive stability. The LUMO of HFE-7100 is lower than that of the carbonate solvents. This indicates that HFE-7100 is more easily reduced a low potentials versus $\mathrm{Li} / \mathrm{Li}^{+}$than the carbonate solvents. Due to similarities in molecular structure to HFE-7100, similar resistance to oxidation and reduction is expected for HFE-7000.

\subsubsection{Method of Measurement}

The electrochemical stability window of an electrolyte is most commonly measured using cyclic voltammetry $(\mathrm{CV})$. The $\mathrm{CV}$ experiment is an extremely versatile technique primarily used by electrochemists to study particular redox reactions. Several of the original instructional papers have been published that describe the fundamentals of the CV experiment [108-110]. The basic test setup for the CV experiment is shown in Figure 4-6. The CV experiment is performed by a potentiostat, which is an instrument that is capable of accurately scanning the potential of a working electrode relative to a reference electrode in a smooth, analog format while simultaneously measuring the current produced at the same working electrode. Charge neutrality is maintained in the electrochemical cell by the use of a counter electrode, whose sole purpose is to supply or sink the electrochemically active species in response to reactions at the working electrode. The reference electrode does not participate in the

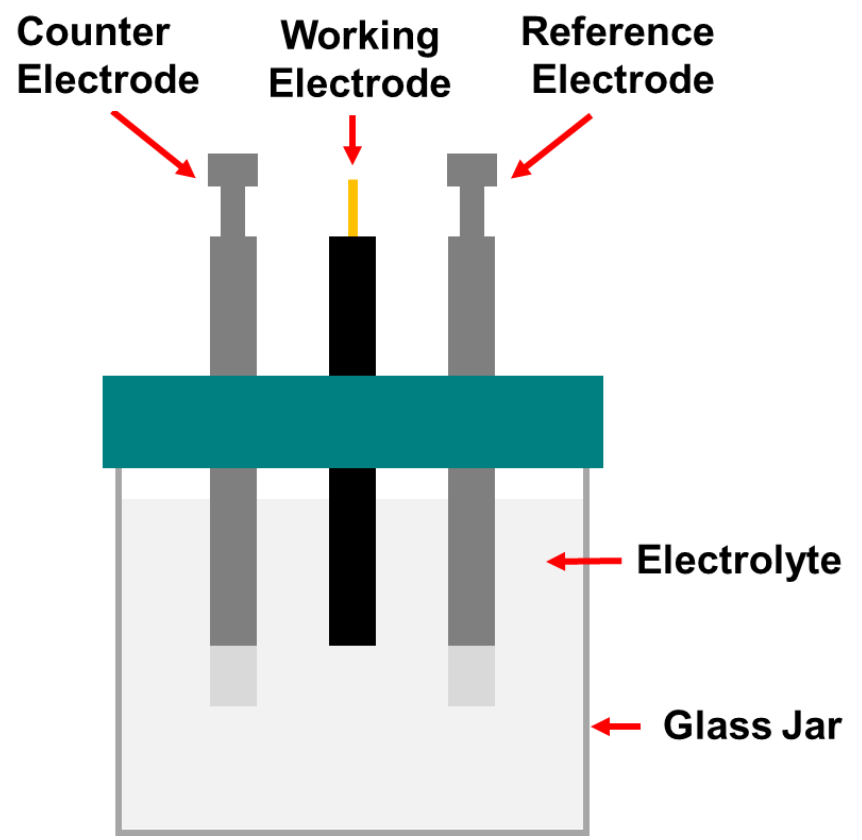

Figure 4-6: 3-Electrode Experimental Setup for CV Experiment 
electrochemical reactions and passes zero current over the entire potential range of the experiment. Therefore, when potentials are measured relative the reference electrode, any changes are the result of electrochemical reactions at the working electrode.

There are several experimental setup considerations for performing an electrochemical stability window CV experiment. The most important consideration is the choice of electrodes. CV requires the use of three electrodes: one working electrode, one counter electrode, and one reference electrode. The next consideration are the switching potentials for the potential scan. The switching potentials are the preset bounds of the electrochemical stability window measurements. The final consideration is the potential scan rate of the instrument performing the experiment. Each of these are now discussed here.

There are many candidate working electrode materials for making electrochemical stability window measurements. In general, all working electrodes used for stability window measurements are inert in the electrolyte solution. Ideally, the only reactions that take place on the electrode surfaces are reactions consistent with the cathodic and anodic stability limits of the electrolyte components. For nonaqueous lithium-ion electrolytes gold, silver, platinum, and glassy carbon are all considered inert electrodes and have been commonly applied for stability window measurements [111-113]. For this work, glassy carbon and platinum working electrodes are used to measure the electrochemical stability window and the resulting windows will be compared, similar to the approach used by Borgel et al. on ionic liquid lithium-ion electrolytes [112].

The counter and reference electrodes used for electrochemical stability window measurements of lithium-ion electrolytes are commonly lithium metal. Lithium metal is a frequent choice because its redox reaction, Equation (1.1), defines the lower operation potential of the lithium-ion chemistry. The solid lithium deposition reaction that occurs at $0 \mathrm{~V} v \mathrm{vs} . \mathrm{Li} / \mathrm{Li}^{+}$is highly 
irreversible in lithium-ion chemistry due to its porous plated structure and can form lithium dendrites capable of shorting a LIB [24]. The formation of lithium dendrites in the lithium-ion chemistry was well visualized in the work of Liu et al. [114]. Therefore, potentials of $0 \mathrm{~V} \mathrm{vs.} \mathrm{Li} / \mathrm{Li}^{+}$ are avoided in lithium-ion chemistry and all components function at potentials greater than the lithium deposition reaction.

Although the counter and reference electrodes are both lithium, their function is very different. The counter electrode participates in the redox reactions of the electrochemically active species in response to the reactions occurring at the working electrode. Counter electrodes should be ideally non-polarizable electrodes, meaning they can pass infinite current and require no overpotential to do so. Lithium metal can be made an approximate non-polarizable electrode by ensuring that its electrochemically active surface area is much greater than that of the working electrode. The use of a lithium metal reference electrode allows for the potential of the working electrode to be scanned within a directly relevant range to the lithium-ion chemistry (i.e., 0.5-5 V vs. $\left.\mathrm{Li} / \mathrm{Li}^{+}\right)$. Other types of reference electrodes can be used, but the applied potential of the working electrode relative to the reference must be converted to the $\mathrm{Li} / \mathrm{Li}^{+}$potential after the experiment is completed.

The switching potentials for a CV electrochemical stability window measurement must ensure that the stability limits of the electrolyte are reached. Desired potential stability windows are guided by the electrode active materials employed in a LIB. The LIB must be capable of reversible operation between the potentials of the electrodes contained in the cell. Accordingly, the electrolyte must have a sufficient stability window to allow for reversible operation of the electrodes. For the lithium-ion chemistry, the upper voltage limit required of the electrolyte is typically $4.5 \mathrm{~V}$ vs $\mathrm{Li} / \mathrm{Li}^{+}$, although there are current research efforts to extend the oxidation 
potential of the electrolyte past this potential using fluorinated solvents [115]. The lower voltage limit of electrochemical stability of conventional electrolyte mixtures is typically between $0.5-1 \mathrm{~V}$ vs. $\mathrm{Li}_{/} \mathrm{Li}^{+}$[22]. Common LIB anode materials reversibly operate below this potential range, but only after the successful formation of a stable SEI [19].

The final test parameter for consideration is scan rate, which is how fast the voltage is swept between the predetermined switching potentials. In CV experiments that study particular redox reactions, varying the scan rate can provide insight into the diffusion coefficient of the active species to the working electrode surface. The measured peak current of the cyclic voltammagram at a particular scan rate corresponds to a mass diffusion limitation. As less time is allowed for the active species to diffuse to the electrode surface at higher scan rates, the peak current predictably changes for carefully-controlled diffusion scenarios. However, electrochemical stability window measurements are not concerned with studying a single redox reaction and varying the scan rate is not a necessary part of the measurement. In addition, scan rate does vary linearly with the measured charging current associated with the double-layer capacitance of the working electrode [29]. This charging current is completely reversible and does not affect the measurement of the stability limits of an electrolyte. This effect is

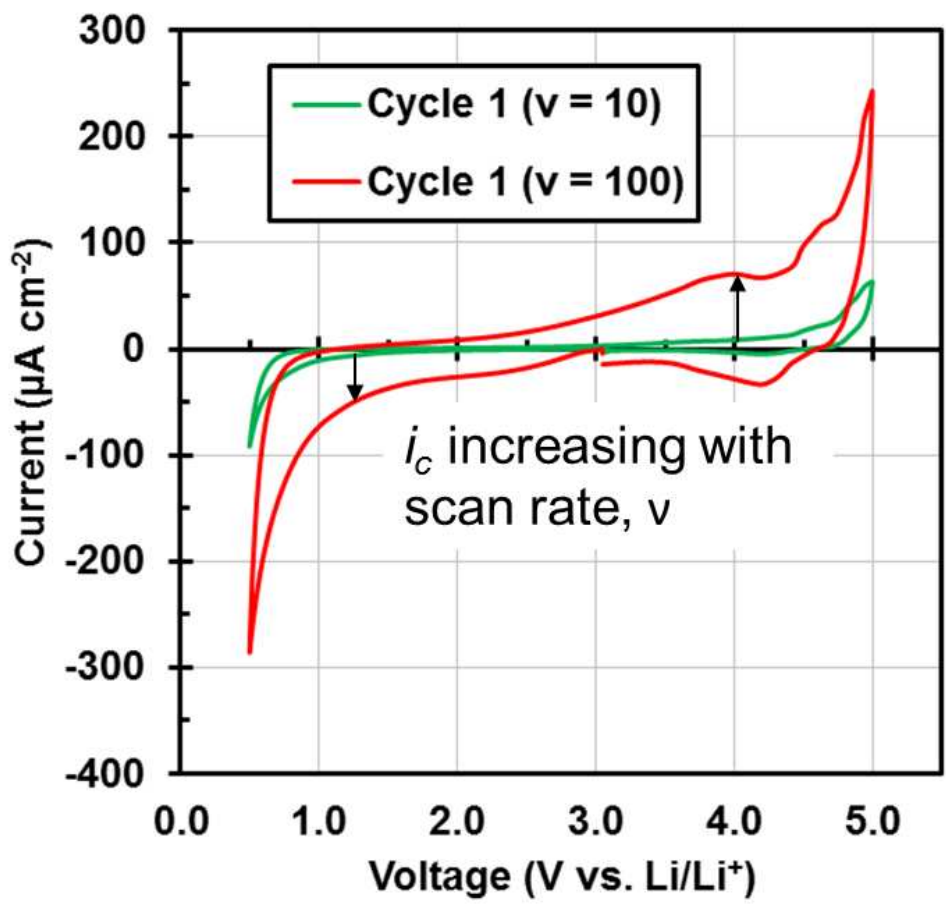

Figure 4-7: Effect of Potential Scan Rate, $v$ in $\mathrm{mV} \mathrm{s}^{-1}$, on Electrochemical Stability Window of E1 Measured with a GCE Working, Lithium Metal Counter and Reference Electrodes 
illustrated in Figure 4-7 which shows the electrochemical stability window of E1 measured on a glassy carbon electrode at two different scan rates, $10 \mathrm{mV} \mathrm{s}^{-1}$ and $100 \mathrm{mV} \mathrm{s}^{-1}$. Although the measured current between the potentials of $0.75-4.75 \mathrm{~V}$ vs. $\mathrm{Li} / \mathrm{Li}^{+}$are much greater for the 100 $\mathrm{mV} \mathrm{s}^{-1}$ scan rate, it does not affect the measurement of oxidation and reduction potentials of the electrolyte that define the stability window. The larger measured current is simply due the charging of the electrochemical double-layer.

\subsubsection{Test Parameters and Equipment Used}

The electrochemical stability window was measured with a Gamry Reference 3000 Potentiostat (Figure 4-8). The Reference 3000 potentiostat has 11 current ranges $(300 \mathrm{pA}-3 \mathrm{~A}$, accuracy of $\pm 5 \mathrm{pA} \pm 0.05 \%$ of range $\pm 0.2 \%$ of value) with $\pm 32 \mathrm{~V}$ capability and a $\pm 1 \mathrm{mV}$ accuracy. Prior to performing the stability window experiments, the instrument was calibrated using the Gamry-provided calibration circuit in a Faraday cage (Figure 4-9). An AC

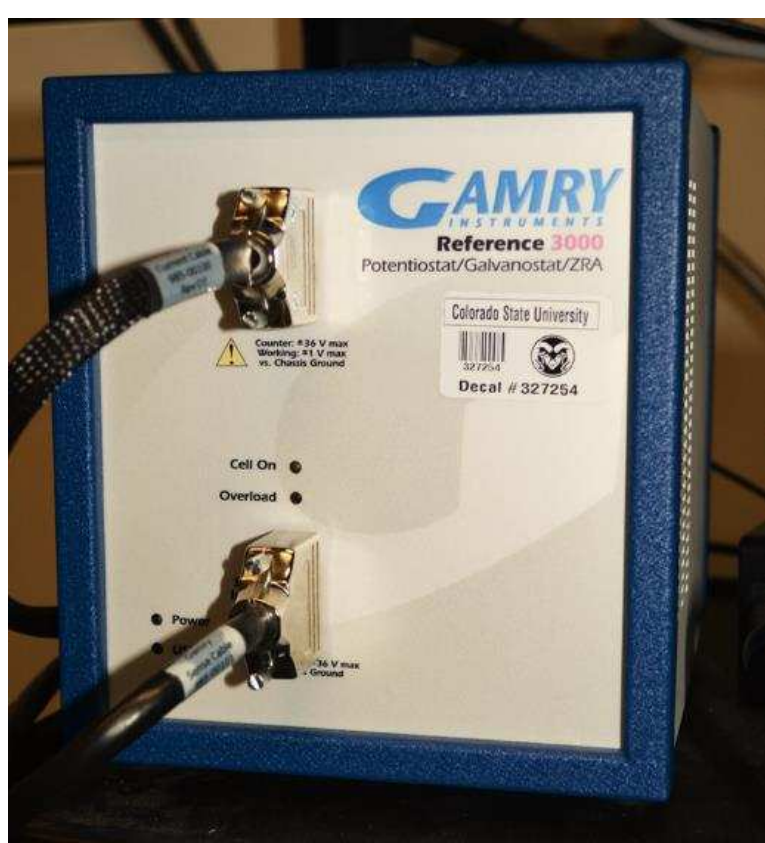

Figure 4-8: Gamry Reference 3000 Potentiostat and DC calibration were performed using the Gamry Framework software built-in utility. The working electrodes were either platinum (1.6 mm diameter, BASi) or glassy carbon (3 mm diameter, BASi) and are shown in Figure 4-10 The counter and reference electrode were lithium metal (Figure 4-11). 


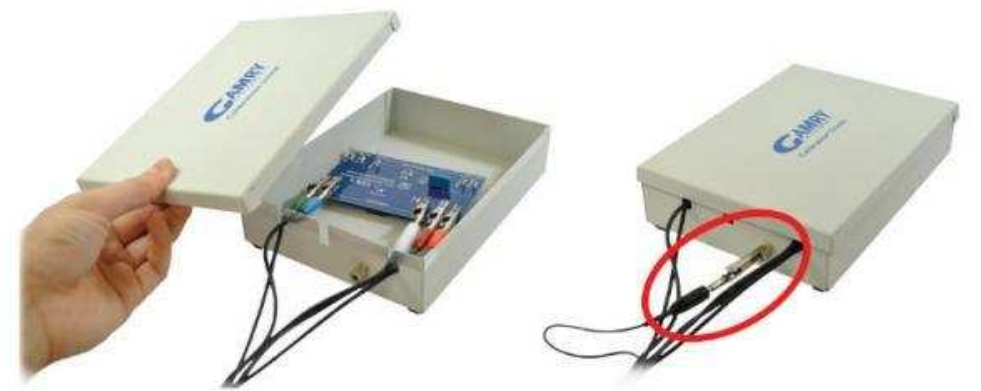

Figure 4-9: Gamry Calibration Circuit and Faraday Cage (source: gamry.com)
The potentiostat cables contain six colored leads with alligator clips (Figure 4-12): working (green), working sense (blue), counter (red), counter sense (orange), reference sense (white),

and ground (black). The cables are hermetically sealed into a glove box feedthrough to allow the instrument to remain outside the glove box to perform the experiment. Both the working and working sense leads were connected to the platinum or glassy carbon working electrode. The counter and counter sense leads were connected to the lithium metal counter electrode. The reference sense lead was connected to the lithium metal reference electrode. The ground lead was connected to the large metal shelves of the argon glove box.

A glass jar lid was modified to accommodate the three electrodes required for this experiment (Figure 4-11). The thickness of the lid was extended using the same epoxy technique as the lid for the conductivity sensor. The working electrodes were purchased pre-embedded in black PEEK plastic, which allowed for direct insertion through the lid. The lithium metal counter and reference electrode required the use of a $6.35 \mathrm{~mm} 316$ stainless steel rod which contained 8-32 female

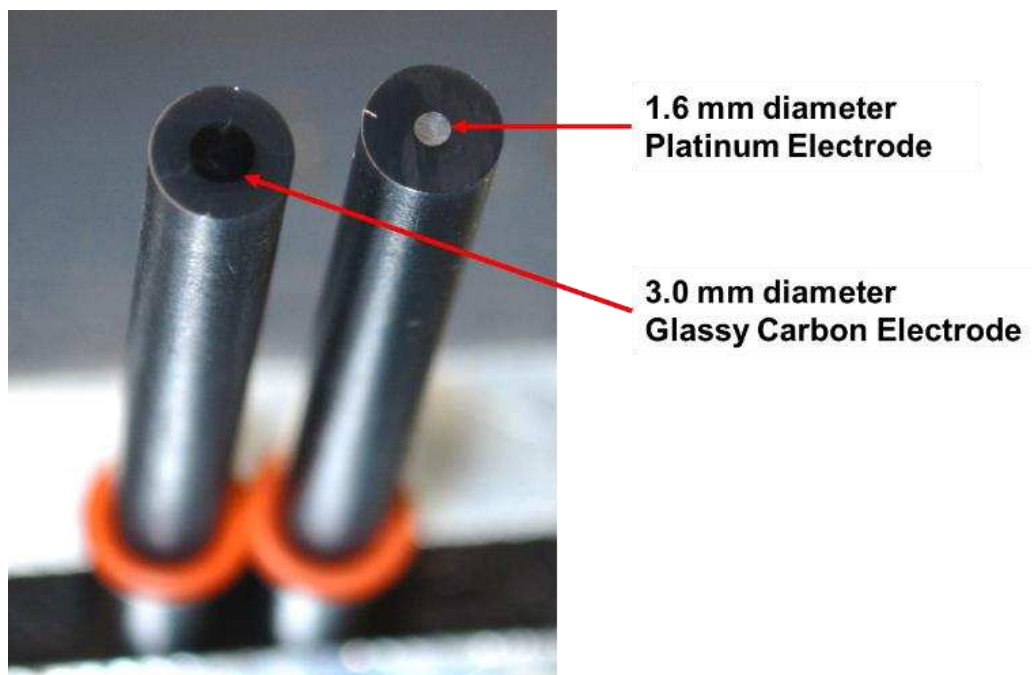
threads on both ends, machined

Figure 4-10: Glassy Carbon and Platinum Working Electrodes Used for Electrochemical Stability Window Measurements 


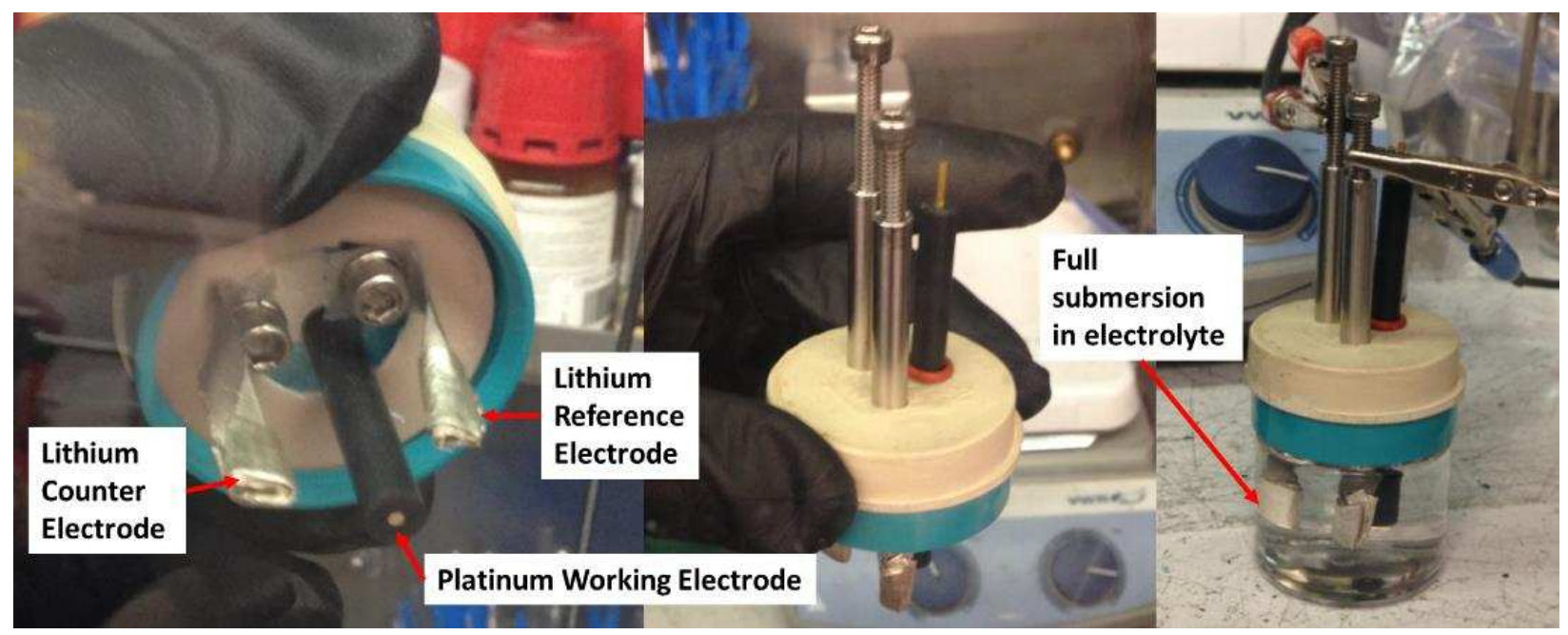

Figure 4-11: Electrochemical Stability Window Experimental Setup with Modified Glass Jar Lid

in-house. A fine, 316 stainless steel mesh is cut into a thin rectangular strip $(7.62 \mathrm{~mm} \times 25.4 \mathrm{~mm})$ and a $4.50 \mathrm{~mm}$ hole is punched on one end. A 316 stainless steel \#8 lock washer and $9.53 \mathrm{~mm}$ long socket head screw tighten the mesh onto the rod through the punched hole to ensure good electrical contact. The lithium metal is then wrapped at the opposite end of the mesh to create the counter and reference electrodes. An additional $25.4 \mathrm{~mm}$ long stainless steel screw is used at the top of the rod outside of the jar to provide an electrical connection location for the alligator clips of the potentiostat leads. Figure 4-11 shows that the surface area of the lithium metal counter electrode is far greater than that of the platinum working electrode to provide an adequate approximation of an ideal non-polarizable electrode.

Prior to testing, all components were cleaned and/or polished. The 316 stainless steel rods were polished with Wenol metal polish using a Kimwipe laboratory tissue. The electrolytewetted portions of the modified lid, rods, screws, lock washers, and mesh were cleaned with acetone,

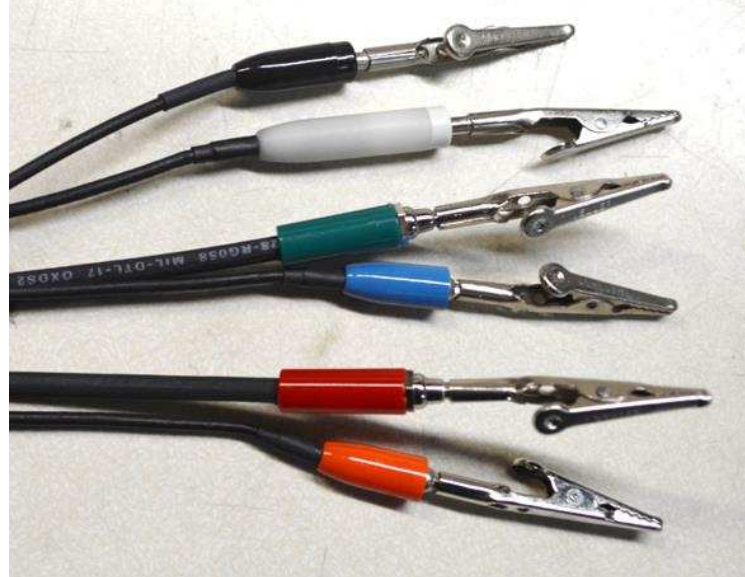

Figure 4-12: Gamry Reference 300 Potentiostat Leads 
methanol, and then isopropanol. The working electrodes were prepared according to the BASi electrode polishing guide [116], described in detail in Appendix B. All components were vacuum dried overnight at $80^{\circ} \mathrm{C}$ prior to pumping into the argon glove box. The lithium metal for the counter and reference electrodes was prepared in the argon glove box. $1 \mathrm{~mm}$ thick $6.35 \mathrm{~mm} \times$ $12.70 \mathrm{~mm}$ rectangles of lithium metal ribbon were cut. A metal spatula was then used to scrape the top oxide surface layer off of the lithium on both sides. The oxide-free lithium has a much greater silver-metallic luster. Finally, the lithium was wrapped on the stainless steel mesh opposite of the screws.

Using the Gamry Framework software interface, the electrochemical stability window parameters were defined. The voltage was scanned at $10 \mathrm{mV} \mathrm{s}^{-1}$ from the open-circuit voltage $(\mathrm{OCV})$ of the working electrode versus the lithium metal reference down to $0.5 \mathrm{~V}$, up to $5 \mathrm{~V}$ vs. $\mathrm{Li} / \mathrm{Li}^{+}$, and back to the OCV (3-3.1 V). The scan was performed for one cycle. The OCV is determined by 30 seconds of potential measurement between the working and reference electrodes prior to sweeping the potential. Figure 4-13 shows the applied potential scan of the potentiostat versus time for one complete electrochemical stability window cycle. The OCV of the working electrode, in this case glassy carbon, was approximately $3.08 \mathrm{~V}$ vs. $\mathrm{Li} / \mathrm{Li}^{+}$. The measured current versus time at the working electrode is also provided. To

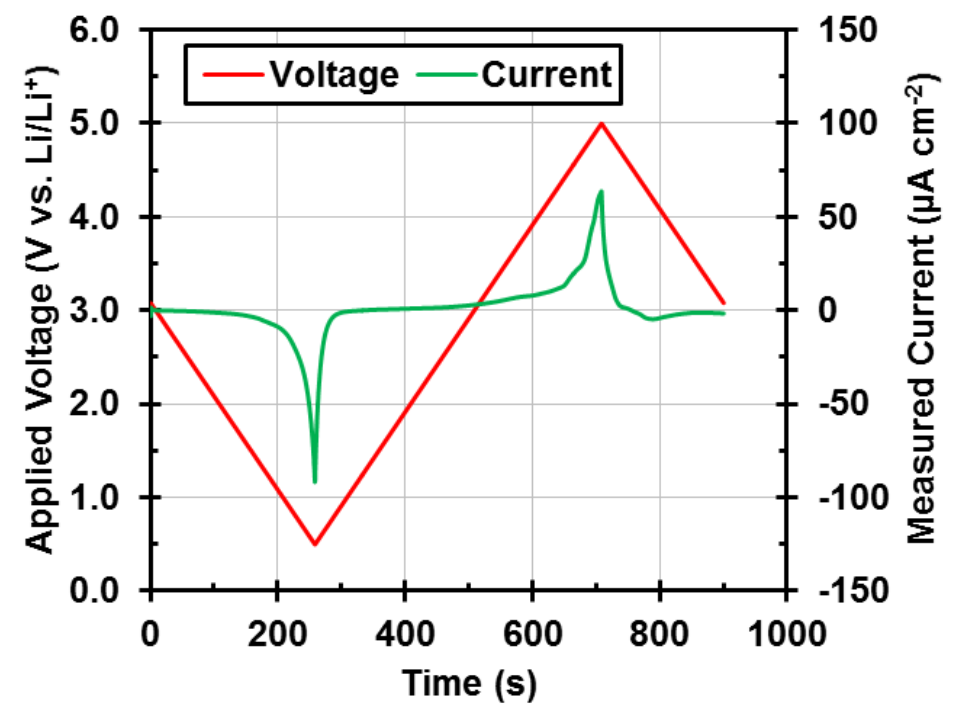

Figure 4-13: Applied Potential Scan and Measured Current during Electrochemical Stability Window Measurement of $\mathrm{E} 1$ at $10 \mathrm{mV} \mathrm{s}{ }^{-1}$ with a Glassy Carbon Working Electrode 
create a cyclic voltammagram, the measured current is plotted against a potential domain, as opposed to a time domain.

\subsubsection{Results and Discussion}

The initial switching potentials selected $\left(0.5\right.$ and $5 \mathrm{~V}$ vs. $\left.\mathrm{Li} / \mathrm{Li}^{+}\right)$proved to be sufficient to capture the stability window of the electrolytes on a glassy carbon electrode (GCE). Both the baseline and E1 reach their oxidative and reductive limits prior to the scan reaching the switching potentials as indicated by the exponential growth and decline in measured current seen in Figure 4-14. As the potential is swept from the OCV towards the lower switching potential $(0.5 \mathrm{~V})$, it can clearly be seen that the baseline electrolyte begins to decompose on the GCE around $1.5 \mathrm{~V}$ vs. $\mathrm{Li} / \mathrm{Li}^{+}$. This observation is consistent with the first lithiation cycle of carbonaceous electrodes which includes significant EC decomposition to form a stable solid electrolyte interphase (SEI) [117]. The candidate electrolyte E1 does not begin to show reductive decomposition until the potential is swept below $1.0 \mathrm{~V}$ vs. $\mathrm{Li} / \mathrm{Li}^{+}$. The baseline electrolyte also showed increased oxidative decomposition at $4.2 \mathrm{~V}$ vs. $\mathrm{Li} / \mathrm{Li}^{+}$.

Both electrolytes appear to reach their oxidative limits by $5 \mathrm{~V}$ vs. $\mathrm{Li} / \mathrm{Li}^{+}$. E1 appears to have improved stability compared to the baseline electrolyte, as less current is passed over the entire potential range until the oxidative and reductive limits are reached. This is a promising result as the baseline

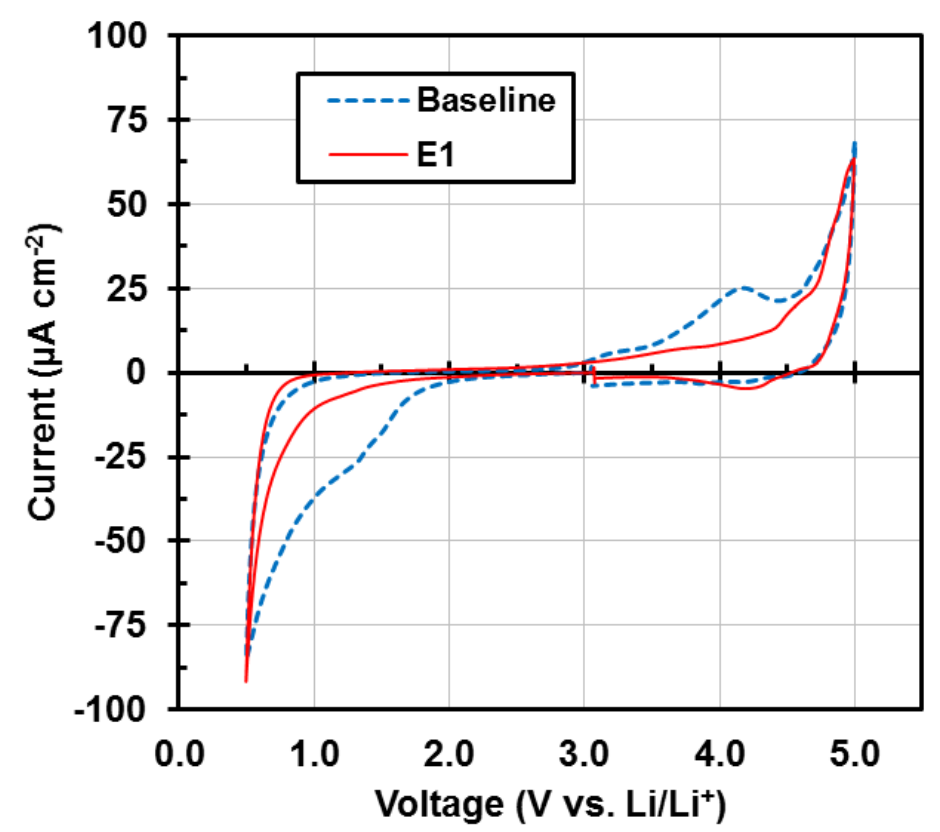

Figure 4-14: Electrochemical Stability Window Measured with a GCE WE, Li metal CE/RE, $10 \mathrm{mV} \mathrm{s}{ }^{-1}$ Scan Rate, $0.5 \mathrm{~V}$ and $5 \mathrm{~V}$ vs. $\mathrm{Li} / \mathrm{Li}^{+}$Switching Potentials 
electrolyte is known to already have a sufficient electrochemical stability with the lithium-ion chemistry due to its ability to form a stable SEI $[19,102]$.

The electrochemical stability window measured on a platinum $(\mathrm{Pt})$ working electrode provided a very different result from the glassy carbon electrode (Figure 4-15). E1 clearly has greater reactivity through the entire scan. The electrochemical stability window of the baseline electrolyte is markedly improved over E1, showing minimal decomposition until the limits of 0.5 and $5 \mathrm{~V}$ vs. $\mathrm{Li}^{2} \mathrm{Li}^{+}$are reached. The increased reactivity of $\mathrm{E} 1$ is most likely due to increased oxygen and water impurities in the electrolyte which are known to have high reactivity on a platinum working electrode [111]. Both the EMC and HFE-7000 fluids were purchased from the suppliers and degassed using the freeze-pump-thaw procedure. Ideally this process removes all trapped gasses, but the results suggest that there are still enough trace amounts of oxygen and water to affect the stability window measurement on platinum. By contrast, the baseline electrolyte was purchased from an electrolyte manufacturer, BASF. The baseline electrolyte mixture was

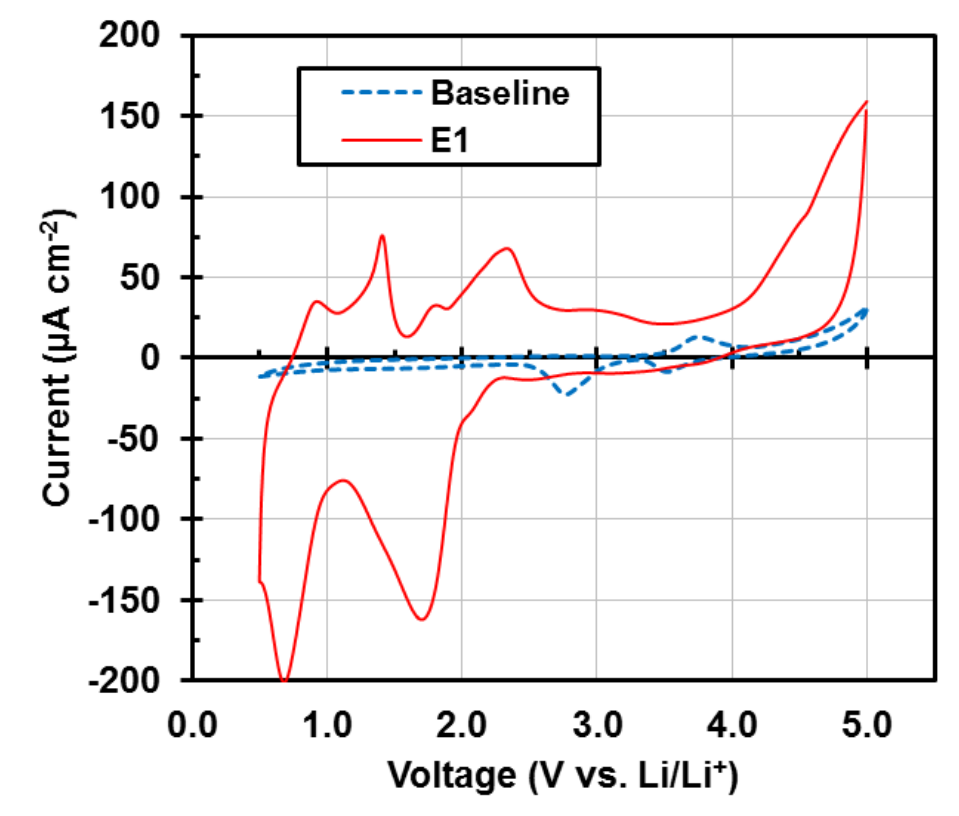

Figure 4-15: Electrochemical Stability Window Measured with a Pt WE, Li metal CE/RE, $10 \mathrm{mV} \mathrm{s}^{-1}$ Scan Rate, $0.5 \mathrm{~V}$ and $5 \mathrm{~V}$ vs. $\mathrm{Li} / \mathrm{Li}^{+}$Switching Potentials

confirmed by the electrolyte manufacturer, BASF, to have $<20$ ppm of $\mathrm{H}_{2} \mathrm{O}$ in the electrolyte. Therefore, the stability window measurement with a platinum working electrode on E1 was more a measure of the impurities contained in the electrolyte than the oxidative and reductive stability limits of the constituents. The stability window 
measured with a glassy carbon electrode does appear to more definitively measure the electrolyte potential limits.

\subsection{Half Cell Electrochemical Testing}

With miscibility, solubility, conductivity, and electrochemical stability of the candidate MFE established, half cell testing was performed to evaluate the electrochemical performance with different LIB active materials with a lithium counter electrode. Half cell testing included galvanostatic cycling and electrochemical impedance spectroscopy. The half cell active materials, construction process, experimental techniques, and resulting data are described in the following sections.

\subsubsection{Definition and Method of Measurement}

Half cells are created by using any LIB active material-containing electrode (cathode or anode) as the working electrode and lithium metal as a counter electrode. The redox potential of the lithium metal counter electrode remains fixed at $0 \mathrm{~V} v \mathrm{vs} . \mathrm{Li} / \mathrm{Li}^{+}$due to having a tremendously larger capacity than the working electrode. Lithium metal electrodes have a theoretical capacity of $3860 \mathrm{mAh} \mathrm{g}^{-1}$, while the capacities of the working electrodes used in this study are all below 350 $\mathrm{mAh}^{-1}[1]$. As opposed to the lithium metal counter electrode, the working electrode changes potential depending upon its state of lithiation. This allows the working electrode to be studied independent of a counter electrode. A half cell containing a LIB active material working electrode and a lithium metal counter electrode is then filled with either the MFE or the baseline electrolyte to complete its construction.

The LIB active materials used to evaluate the two electrolytes in half cells are lithium iron phosphate ( $\mathrm{LFP}, \mathrm{LiFePO}_{4}$ ), lithium titanate oxide (LTO, $\mathrm{Li}_{4} \mathrm{Ti}_{5} \mathrm{O}_{12}$ ), and copper antimonide $\left(\mathrm{Cu}_{2} \mathrm{Sb}\right)$. LFP is a common active material in LIBs and is the only cathode active material used in 
this work. $\mathrm{LTO}$ and $\mathrm{Cu}_{2} \mathrm{Sb}$ are two LIB anode active materials that serve as alternatives to graphite, the most common anode active material in commercial LIBs. The electrochemical characteristics, benefits, and drawbacks for each active material are discussed here.

LFP is one of the most common high power LIB cathode active materials. The electrochemical reaction of LFP is a constant voltage, two-phase process described by the reversible reaction as follows:

$$
\mathrm{LiFePO}_{4} \underset{\text { Lithiation }}{\stackrel{\text { Delithiation }}{\rightleftharpoons}} \mathrm{FePO}_{4}+\mathrm{Li}^{+}+e^{-}
$$

This reaction occurs at $3.5 \mathrm{~V}$ vs. $\mathrm{Li} / \mathrm{Li}^{+}$with a theoretical capacity of $170 \mathrm{mAh} \mathrm{g}^{-1}$. In addition, the lithiation process only produces a unit cell volume expansion of $6.81 \%$. The minimal volume expansion of the LFP particle minimizes the amount of capacity degradation due to SEI cracking on the particle surface and subsequent need for reformation, which enables long cycle life [118]. Moreover, the redox potential of $3.5 \mathrm{~V}$ vs. $\mathrm{Li} / \mathrm{Li}^{+}$, although lower than other popular cathode active materials, is a lower chemical potential than the highest occupied molecular orbital (HOMO) of conventional organic carbonate electrolytes. This minimizes the likelihood of a passivation layer (SEI) forming on the surface of the LFP particles. The binary mixture of the MFE does not contain any additives commonly associated with stable SEI formation [22]. Therefore, the preclusion of SEI formation offers a valuable first-look at the electrochemical performance of the MFE without complicated and irreversible reactions associated with the SEI. Finally, LFP is inexpensive, nontoxic, and a thermally stable active material [119].

LTO is a highly reversible anode for the LIB chemistry, typically employed for high power applications. The high reversibility is due to two unique features of the active material: a flat operation voltage of $1.5 \mathrm{~V}$ vs. $\mathrm{Li} / \mathrm{Li}^{+}$and a near zero lattice expansion and contraction upon 
lithiation and delithiation. The reversible two-phase reaction of LTO that delivers a theoretical capacity of $175 \mathrm{mAh} \mathrm{g}^{-1}$ is as follows:

$$
\mathrm{Li}_{4} \mathrm{Ti}_{5} \mathrm{O}_{12}+3 \mathrm{Li}^{+}+3 e^{-\underset{\text { Delithiation }}{\stackrel{\text { Lithiation }}{\rightleftharpoons}}} \mathrm{Li}_{7} \mathrm{Ti}_{5} \mathrm{O}_{12}
$$

Analogous to LFP, the flat voltage plateau of LTO at $1.5 \mathrm{~V}$ vs. $\mathrm{Li} / \mathrm{Li}^{+}$is above the chemical potential of the LUMO of the electrolyte solvents. At this high of a potential, theoretically there is no solvent reduction and SEI formation. Furthermore, the high potential of LTO thermodynamically eliminates the possibility of lithium metal plating [120]. LTO particles have inherently low electronic conductivity, a property that can be mitigated by small active material particle size. Taking this idea to the extreme, Kavan and coworkers reported satisfactory electrochemical performance of a nanocrystalline LTO thin film electrode at charging rates of 250C [121]. For these reasons, LTO is considered a safe, high-rate anode material for LIBs.

$\mathrm{Cu}_{2} \mathrm{Sb}$ is an intermetallic anode capable of reversible $\mathrm{Li}^{+}$insertion and extraction. Intermetallic anodes offer several benefits compared to other types of negative electrodes for LIBs. As opposed to insertion electrodes like LFP, LTO, and graphite which store lithium in atomic form, intermetallic anodes store lithium in ionic form. This enables some of the lithium intermetallic alloys to have specific capacities on the order of lithium metal. In addition, intermetallic anodes have higher potentials versus lithium than graphite, which reduces the likelihood of lithium plating on the surface of the anode. Lithium can be alloyed with numerous different metalloids including antimony (Sb). However, intermetallic anodes undergo significant volume expansion and contraction during lithiation and delithiation (100-200\%). The expansion causes cracking both of the active material and the SEI and loss of surrounding particle electric contact [15]. $\mathrm{Cu}_{2} \mathrm{Sb}$ is an intermetallic anode of particular interest because its volume expansion is much less significant, $42 \%$ [17], than other intermetallic alloys. In addition, $\mathrm{Cu}_{2} \mathrm{Sb}$ has a high 
theoretical capacity of $323 \mathrm{mAh} \mathrm{g}^{-1}$, comparable to that of graphite (372 $\left.\mathrm{mAh} \mathrm{g}^{-1}\right)$. The lithiation of $\mathrm{Cu}_{2} \mathrm{Sb}$ is described by the following complete reaction:

$$
\mathrm{Cu}_{2} \mathrm{Sb}+3 \mathrm{Li}^{+}+3 e^{-} \underset{\text { Delithiation }}{\stackrel{\text { Lithiation }}{\rightleftharpoons}} L i_{3} \mathrm{Sb}+2 \mathrm{Cu}
$$

In the complete reaction, copper is extruded from the intermetallic structure. Pure $\mathrm{Cu}_{2} \mathrm{Sb}$ upon initial lithiation undergoes two separate reactions. The first two-phase reaction is described as:

$$
C u_{2} S b+x \mathrm{Li}^{+}+x e^{-} \underset{\text { Delithiation }}{\stackrel{\text { Lithiation }}{\rightleftharpoons}} L i_{x} C u_{2-y} S b+y C u \text { for } 0<x \leq 2 \text { and } 0 \leq y \leq 1
$$

At the limits of the reaction described by Equation (4.4), $\mathrm{Li}_{2} \mathrm{CuSb}$ is formed. Upon further lithiation, the electrode undergoes a second single-phase reaction:

$$
L i_{2+z} C u_{1-z} S b+z L i^{+}+z e \underset{\text { Delithiation }}{\stackrel{\text { Lithiation }}{\rightleftharpoons}} L i_{3} S b+z C u \text { for } 0<z \leq 1
$$

The potentials at which the above reactions for $\mathrm{Cu}_{2} \mathrm{Sb}$ take place are $0-1 \mathrm{~V}$ vs. $\mathrm{Li} / \mathrm{Li}^{+}$, with the reaction described in Equation (4.5) solely occurring at potentials less than $0.5 \mathrm{~V}$ vs. $\mathrm{Li} / \mathrm{Li}^{+}$[17]. Note that in the complete reaction described by Equations (4.3) and (4.5) all the antimony is reacted with lithium, leaving copper extruded from the structure. The formation of $\mathrm{Li}_{3} \mathrm{Sb}$ is unfavorable for reversible cycle life, therefore $\mathrm{Cu}_{2} \mathrm{Sb}$ anodes are typically lithiated to a minimum of $0.5 \mathrm{~V}$ vs. $\mathrm{Li} / \mathrm{Li}^{+}$to avoid complete copper extrusion.

The construction of a LFP half cell is shown in Figure 4-16 and Figure 4-17. A 19 mm PFA T-fitting was used as the body of the cell. The PFA T-fittings were purchased from Entegris (part number UT12N) and bored through using a $19 \mathrm{~mm}$ drill bit. $19 \mathrm{~mm}$ diameter 6061 aluminum and 316 stainless steel rods entered the T-fitting at opposite ends of the bored passage and make direct electrical contact with the working and counter electrodes, respectively. Two ferrulecontaining PFA nuts provided a satisfactory seal between the rod and the PFA T-body. For all half cells, the counter electrode was a $19 \mathrm{~mm}$ diameter $\times 1 \mathrm{~mm}$ thick disk of lithium metal placed 


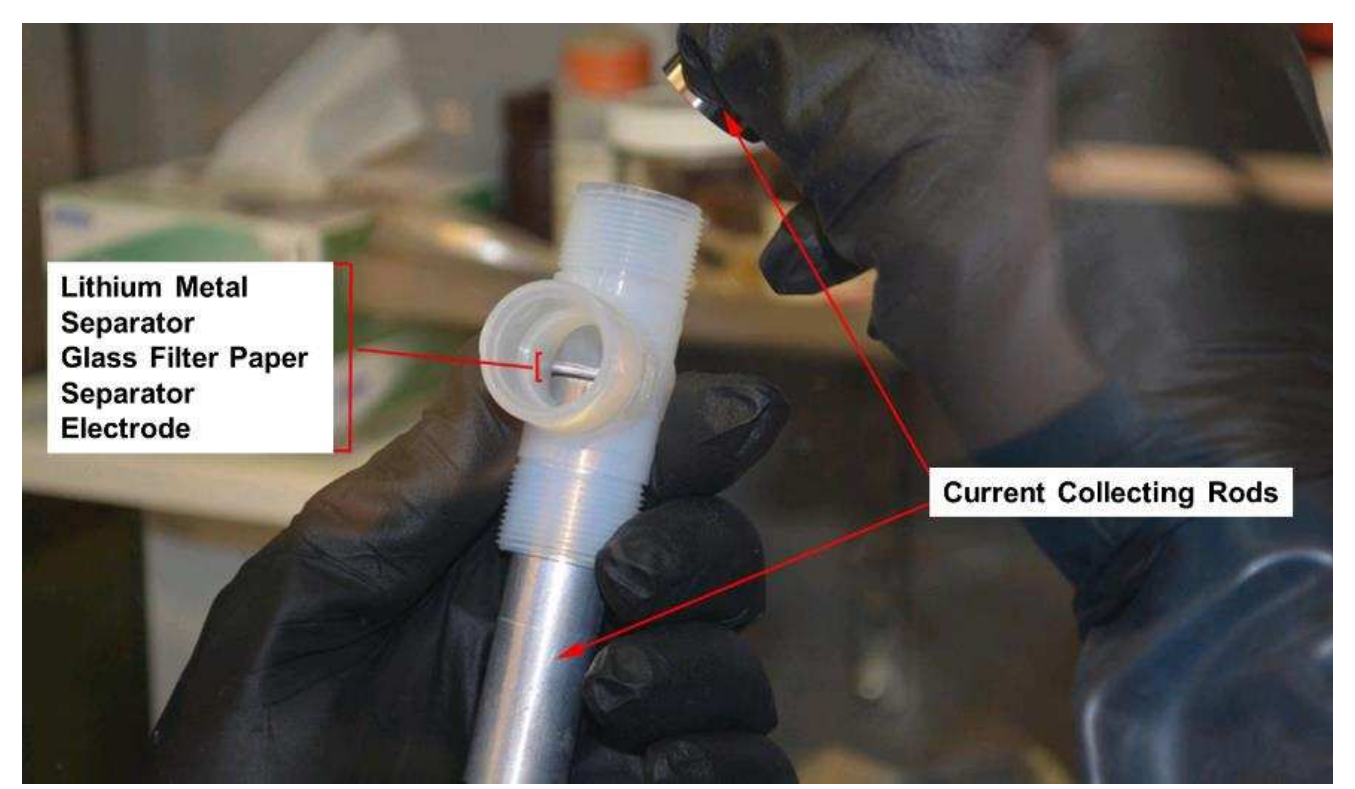

Figure 4-16: Half Cell Stacking Architecture Performed in Argon Glove Box

directly in contact with the stainless steel current collecting rod. Prior to assembly, the surface oxides of the lithium disk were scraped with a metal spatula. In addition, the mass and thickness of the working electrode was measured and recorded using a Mettler Toledo scale with $0.1 \mathrm{mg}$ accuracy and a digital micrometer ( $1 \mu \mathrm{m}$ precision), respectively. A $19 \mathrm{~mm}$ diameter $25 \mu \mathrm{m}$ thick PP/PE separator (MTI) was placed directly on top of the lithium. A $19 \mathrm{~mm}$ diameter piece of glass filter paper was then placed on top of the separator. Another piece of separator was placed on top of the filter paper and the LFP working electrode. The opposite side of the LFP electrode was in direct contact with the aluminum current collecting rod. An identical stacking structure was used for the LTO and $\mathrm{Cu}_{2} \mathrm{Sb}$ half cells. However, these cells used $19 \mathrm{~mm} 101$ copper rods in place of the aluminum rods. Approximately $1 \mathrm{~mL}$ of the candidate MFE or baseline electrolyte was inserted into the top of the T-fitting directly over the stacked electrode structure. A cap was then tightened on the top port of the T-fitting to seal the cell.

Each of the electrode materials in half cells were investigated using galvanostatic cycling and electrochemical impedance spectroscopy (EIS). Both of these experimental techniques are described in the following sections. 


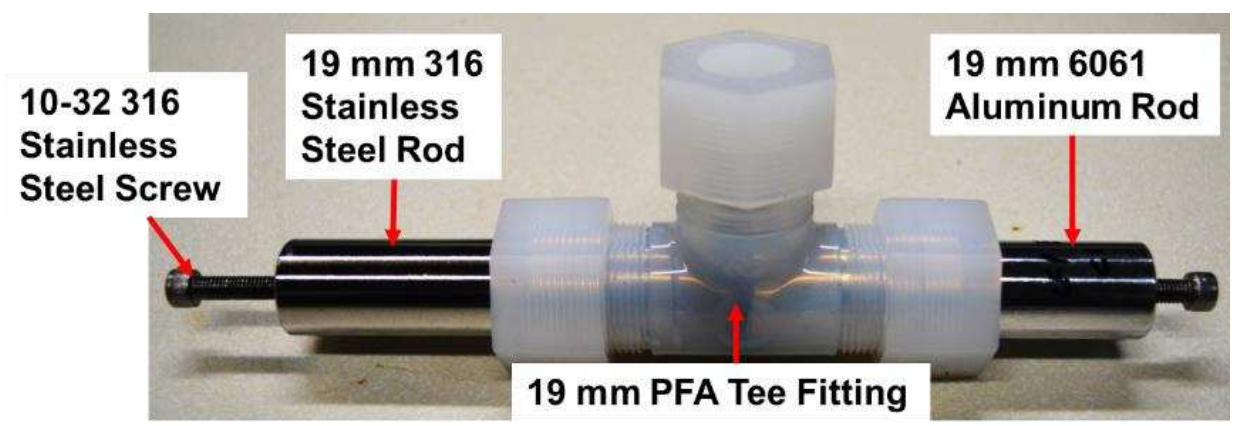

Figure 4-17: Assembled LFP Half Cell

\subsubsection{Galvanostatic Cycling}

Galvanostatic, or constant-current, cycling is the most common assessment tool for evaluating the performance of a battery. The cell under test has either current drawn or input to the cell until a pre-determined time or voltage limit is reached. The resulting data of cell voltage, current input or drawn, and time allows for several important parameters to be calculated including capacity and energy. Capacity is calculated using the following integral:

$$
C(t)=\int_{0}^{t} I(t) d t
$$

where $C(t)$ is the capacity in amp-hours (Ah), I(t) is the applied current in amps (A), and $t$ is time in hours. For a galvanostatic test, the applied current is constant, within limits of instrument accuracy. It is necessary to utilize the recorded current at every data point, $I(t)$, to maximize accuracy. The change in capacity of a cell over many cycles provides insight into the reversibility of the power-producing redox reactions of the electrodes. The energy of the cell is calculated using the following integral:

$$
E(t)=\int_{0}^{t} V(t) I(t) d t
$$

where $E(t)$ is the energy in watt-hours (Wh) and $V(t)$ is the voltage of the cell at time $t$, in hours. 
The data from the galvanostatic tests can be further manipulated to produce relevant information. In particular, the voltage versus state-of-lithiation and the coulombic efficiency of the electrodes are useful to assess the impact of the two electrolytes. The state-of-lithiation $(S O L)$ of an electrode was determined using Equation (4.8) and Equation (4.9) for the cathode and anode, respectively:

$$
\begin{gathered}
S O L_{L F P}=\frac{C(t)}{C_{\text {delithiation }}} \cdot 100 \\
S O L_{C u_{2} S b, L T O}=\frac{C(t)}{C_{\text {lithiation }}} \cdot 100
\end{gathered}
$$

Cathode active materials require energy input for the delithiation reaction, while anode active materials require energy for the lithiation reaction. The normalized capacity data ranged from 0 to 100 percent $S O L$ and was plotted against the measured cell voltage to provide insight into the capacity producing reactions and the overpotentials required for lithiation and delithiation. Similar to the $S O L$ calculations, the coulombic efficiency $(C E)$ was calculated using one of the two formulas depending if the electrode of study was a cathode, Equation (4.10), or anode, Equation (4.11):

$$
\begin{gathered}
C E_{L F P}=\frac{C_{\text {lithiation }}}{C_{\text {delithiation }}} \cdot 100 \\
C E_{C U_{2} S b, L T O}=\frac{C_{\text {delithiation }}}{C_{\text {lithiation }}} \cdot 100
\end{gathered}
$$

The coulombic efficiency calculations provided insight into the reversibility of the capacityproducing electrode processes. A low coulombic efficiency indicated that lithium-ions are trapped in the active material and are unable to be released. High coulombic efficiency indicated high reversibility: all the intercalated lithium is de-intercalated. The comparison of coulombic 
efficiency between the baseline and MFE in each of the half cell combinations allowed for direct conclusions to be made on the performance and feasibility of the MFE in lithium-ion chemistry.

\subsubsection{Electrochemical Impedance Spectroscopy}

Electrochemical impedance spectroscopy (EIS) is an experimental technique that provides valuable insight into the change of impedance at the electrode-electrolyte interface. The impedance of this interface is composed of several different, well-understood electrochemical components. EIS is of direct contrast to cyclic voltammetry and galvanostatic cycling which force nonequilibrium conditions upon an electrochemical system and observe the response of the system. Further description of the purpose of the EIS technique is provided in the following section. Thereafter, the experimental considerations and method for performing an EIS experiment are discussed. The analysis of the resultant impedance data is then explained, including the use of equivalent circuit models. An example of a Randles Cell is provided for further clarification throughout the description of the analysis procedure. Finally, the equivalent circuit model used for impedance fitting in half cell testing is described. Useful textbooks to reference on the subject of EIS are written by Barsoukov and Macdonald [122] and Orazem [123].

\subsubsection{Purpose}

EIS is capable of observing impedance changes at the electrode-electrolyte interface of an electrochemical cell. Specifically, EIS measurements are capable of quantifying the electronic and ionic conduction resistances of the cell, the electrochemical double-layer, the charge-transfer resistance of SEI films on the electrode surface, and the mass diffusion characteristics of the active

materials into the electrode and tracking how each change with potential and cycle life. Each of the aforementioned electrochemical components produce impedance at different timescales. Electronic and ionic conductivity impedance is observed at the smallest micro-second and below 
time scales. Double-layer and SEI charge transfer impedance is observed at the milli-second time scale. Lastly, mass transfer impedance is observed on the second time scale [123]. EIS is capable of characterizing each of these electrochemical components because impedance measurements can be made in the frequency range of $10^{-4}$ to $10^{6} \mathrm{~Hz}$ [29].

It is important to note that the impedance of an electrochemical cell varies for every system chemistry (electrodes and electrolyte) and morphology (structure of the electrode-electrolyte interface). For this reason, EIS is only a useful technique for observing changes to an electrochemical cell with a particular chemistry and morphology. Of interest in this work are the electronic and ionic conduction resistances of the half cell, the electrochemical double-layer, and the charge-transfer resistance of the SEI films on the surface of the electrodes. Each of these are significantly impacted by the components of the electrolyte, especially the resistance associated with ionic conductivity and the SEI film. The resulting impedance data of half cells with E1 will be directly compared to cells containing the baseline electrolyte to assess the impact of the candidate electrolyte mixture on each of the previously mentioned electrochemical components.

The EIS experiment operates by applying a small potential perturbation to the electrochemical cell over a set frequency range. The current produced due to the potential perturbation is measured. The impedance of the cell is determined by the magnitude and phase of the measured current relative to the applied potential. The resulting impedance data can be modeled with equivalent electrical circuits to quantify each of the electrochemical features at the electrode-electrolyte interface. Tracking the changes in the equivalent electrical circuit fits of the impedance data over the lifetime of the electrochemical cell provides insight into the stability or instabilities present at the interface. The electrolyte is a critical component in determining the 
properties of this interface and the EIS data will be useful in evaluating the impact of the MFE on its different components.

\subsubsection{Experimental Considerations and Method}

There are several critical experimental considerations that must be met prior to performing an EIS experiment on a half cell. The most important is ensuring that the cell is maintained at a constant, time-averaged state of charge. The polarization curve of a half cell is the potential of the working electrode relative to lithium metal at any state of lithiation under zero current. Ensuring the cell remains at the same nominal potential during the experiment allows for the impedance of the cell to be analyzed as a linear circuit. Linear circuits can be analyzed with the principle of superposition: inputs to the system are equivalent to the weighted sum of the outputs [124]. Superposition only applies to electrochemical cells for very small potential perturbations. In this

work, EIS experiments are performed potentiostatically (a constant DC potential offset) to approximate steady-state conditions. Immediately prior to the EIS experiment, the half cells under study are lithiated or delithiated to the potential $\left(V_{\mathrm{DC}}\right)$ defined by the potentiostatic test condition. Throughout the remainder of the EIS experiment, the sinusoidal AC potential perturbation, $\Delta V \cos (\omega t)$, is offset by the DC potentiostatic test condition (i.e., $V_{\text {cell }}=\Delta V \cos (\omega t)+V_{\mathrm{DC}}$ ).

The second critical experimental consideration is the magnitude of the potential perturbation applied to the electrochemical cell. For most electrochemical systems a potential perturbation with an amplitude of 1 to $10 \mathrm{mV}$ is sufficient [123]; potential perturbations greater than these will induce a non-linear response, and anything less than $1 \mathrm{mV}$ will measure increased noise from the instrument and obscure the impedance data of the cell. In the present study, a perturbation amplitude of $5 \mathrm{mV}_{\mathrm{rms}}(7.07 \mathrm{mV})$ is used. 
The final critical experimental considerations deal with managing electromagnetic interference (EMI). EMI is the result of the current-magnetic field relationship described by Ampère's Law, which states a magnetic field, $B$, exists perpendicular to the direction of an enclosed current, $I_{\text {enc: }}$

$$
\oint_{C} B \cdot d l=\mu_{0} I_{\mathrm{enc}}
$$

Where $l$ is an arbitrary unit of length and $\mu_{0}$ is the magnetic permeability in vacuum. EMI is present in the test leads of the instrument performing the EIS experiment and the leads of the electrochemical cell under study. The EMI in the test leads can be mitigated by the use of shielded cables; the EMI in the leads of the electrochemical cell must be dealt with in the cell design. Figure 4-17 shows that the cell electrical lead connections are made with concentric 10-32 screws threaded into $19 \mathrm{~mm}$ circular rods. Any EMI induced by the passing of current through these leads will be in the same orientation and the effects are minimized. Further isolation from environmental EMI can be achieved with the use of a Faraday cage. EMI can be produced from the $60 \mathrm{~Hz} \mathrm{AC}$ electric line frequency of nearby powerlines and lights. Faraday cages are electrically connected to an earth ground and absorb all the environmental EMI. In the present study, a Faraday Cage was not used because of the sufficient distance of the cells from ceiling lights and electrical lines.

The EIS experimental method has briefly been introduced in the previous section. A more thorough explanation with relevant equations is now provided. The electrochemical cell under study is electrically connected to the EIS instrument. After the EIS experiment begins, the applied potential perturbation to the working electrode versus the counter electrode is described by Equation (4.13):

$$
V(t)=\Delta V \cos (\omega t)
$$


Where $\Delta V$ is the amplitude of the potential perturbation of the system in volts and $\omega$ is the angular frequency in $\mathrm{rad} \mathrm{s}^{-1}$ of the applied signal. Equation (4.14) relates the angular frequency, $\omega$ to the frequency in $\mathrm{Hz}, f$ :

$$
f=\frac{\omega}{2 \pi}
$$

The applied signal is stepped through the set frequency cutoffs defined by the experiment. The number of impedance data points desired sets the number of independent potential perturbations that are applied to the system.

For each frequency set point of the applied potential perturbation signal, the following measurements and analysis of impedance ensues by the instrument. The measured current at the working electrode due to the applied potential described in Equation (4.13) is:

$$
I(t)=I \cos (\omega t+\phi)
$$

Where $I$ is the amplitude of the measured current signal in amps and $\phi$ is the phase angle of the measured current in radians. The in-phase or out-of-phase response of the measured current at a particular frequency is what provides insight to each of the different characteristic time constants of the half cells under study. By direct extension of Ohm's Law to AC impedance, the following relationship is defined:

$$
V(t)=I(t) \cdot Z
$$

Where $Z$ is the complex impedance of the system. Rearranging Equation (4.16) for $I(t)$ and substituting into Equation (4.15) gives the following expression:

$$
I(t)=\frac{\Delta V}{Z} \cos (\omega t+\phi)
$$

Impedance can be expressed in several different notations, the most common of which is in rectangular complex notation: 


$$
Z(\omega)=Z_{\mathrm{r}}-j Z_{\mathrm{j}}
$$

where $Z_{\mathrm{r}}$ is the real part of the impedance and $Z_{\mathrm{j}}$ is the imaginary part. The imaginary part of the impedance is multiplied by the imaginary unit $j$, defined as the $\sqrt{-1}$ for the complex notation. It is important to note that impedance, $Z$, is only a function of angular frequency. That means each impedance data point obtained from an EIS experiment has a unique frequency. Euler's formula can be used to relate complex and trigonometric functions useful for analysis of the impedance data:

$$
Z(\omega)=\frac{V(t)}{I(t)}=Z e^{j \omega t}=Z(\cos \omega t+j \sin \omega t)
$$

Equation (4.19) is particularly useful to separate the real and imaginary components of impedance in the measured time domain of voltage and current for a single-frequency Fourier transform. The Fourier transform converts the time domain of the voltage and current signals to a frequency domain of impedance, as seen in Equations (4.20)-(4.23):

$$
\begin{gathered}
I_{\mathrm{r}}(\omega)=\frac{1}{T} \int_{0}^{T} I(t) \cos (\omega t) d t \\
I_{\mathrm{j}}(\omega)=-\frac{1}{T} \int_{0}^{T} I(t) \sin (\omega t) d t \\
V_{\mathrm{r}}(\omega)=\frac{1}{T} \int_{0}^{T} V(t) \cos (\omega t) d t \\
V_{\mathrm{j}}(\omega)=-\frac{1}{T} \int_{0}^{T} V(t) \sin (\omega t) d t
\end{gathered}
$$

Where $T$ is the period of the signal in seconds, defined as the inverse of the applied frequency:

$$
T=\frac{1}{f}
$$


The imaginary portions of the applied potential and measured current can then be divided to produce the real and imaginary portions of impedance:

$$
\begin{aligned}
& Z_{\mathrm{r}}(\omega)=\operatorname{Re}\left\{\frac{V_{\mathrm{r}}+j V_{\mathrm{j}}}{I_{\mathrm{r}}+j I_{\mathrm{j}}}\right\} \\
& Z_{\mathrm{j}}(\omega)=\operatorname{Im}\left\{\frac{V_{\mathrm{r}}+j V_{\mathrm{j}}}{I_{\mathrm{r}}+j I_{\mathrm{j}}}\right\}
\end{aligned}
$$

Note Equations (4.25) and (4.26) are Ohm's Law in complex notation. The resulting real and imaginary components of impedance are in the units of Ohms. The phase angle can be related directly to the resulting real and imaginary components using the following relationship:

$$
\tan (\phi)=\frac{Z_{\mathrm{j}}}{Z_{\mathrm{r}}}
$$

The process of applying the varying frequency potential perturbation, measuring the resulting current, and determining the impedance at each frequency is done by the EIS test instrument. The analysis of the resulting data is now discussed with a Randles Cell example.

\subsubsection{Analysis of Resultant Data with Randles Cell Example}

After the completion of an EIS experiment, the resulting data contains the modulus $\left(Z_{\text {mod }}\right)$, phase $\left(Z_{\mathrm{phz}}\right)$, and frequency $(f)$ of the measured impedance. The modulus of the impedance is related to the real and imaginary portions by the following equation and has units of Ohms:

$$
Z_{\text {mod }}=\sqrt{Z_{\mathrm{r}}^{2}+Z_{\mathrm{j}}^{2}}
$$

$Z_{\mathrm{phz}}$ is related to the real and imaginary portions of impedance by Equation (4.27) and has units of degrees. Splitting the modulus of impedance into real and imaginary components produces an alternative way to display the data. Figure 4-18 shows a 3D plot of the impedance of a Randles Cell with the frequency, real, and imaginary portions of the data displayed on the $\mathrm{x}, \mathrm{y}$, and $\mathrm{z}$ axes 


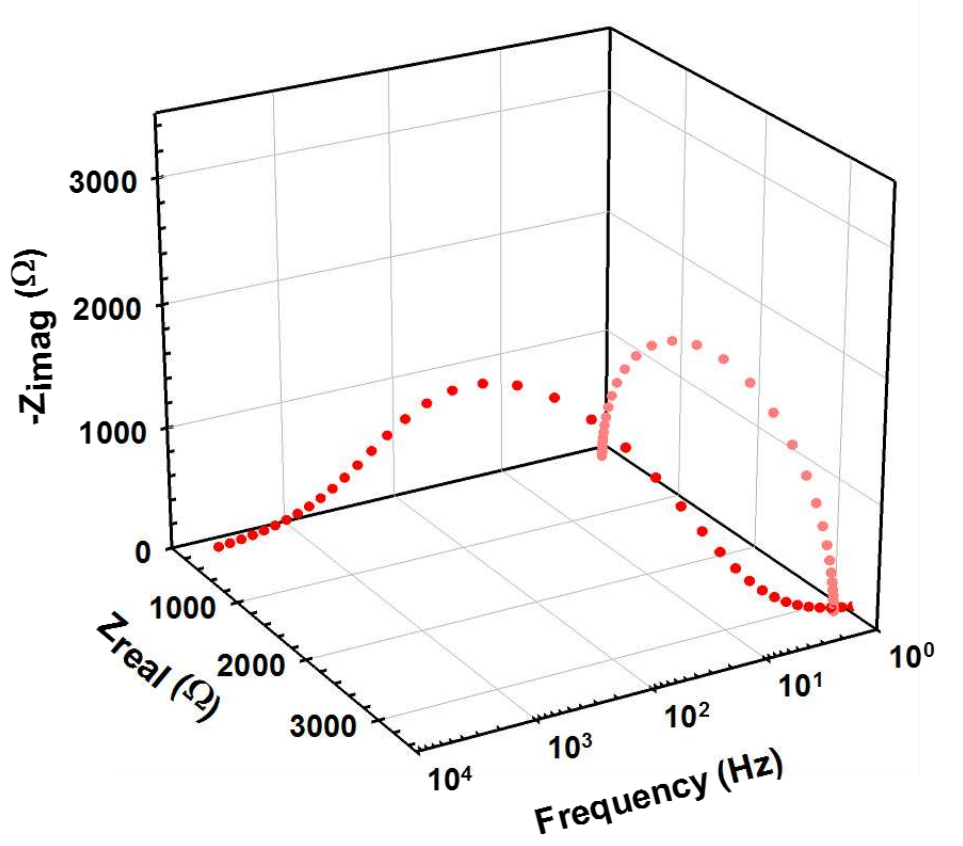

Figure 4-18: Impedance Spectra of a Randles Cell from $1 \mathrm{~Hz}-5 \mathrm{kHz}$ respectively. Note that the lighter colored red dots are a projection of the impedance data onto the $y-z$ plane. The electrical circuit schematic of the Randles Cell used to generate the impedance data seen in the 3D plot is shown in Figure 4-19. A Randles Cell is a RC circuit composed of $R_{\mathrm{ct}}$ and $C_{\mathrm{dl}}$ in series with a resistor, $R_{\Omega}$. The Randles Cell is one of the most basic

electrochemical cell models and is often the foundation for more complex electrochemical system modeling. In this instance, $R_{\Omega}$ represents the cell/solution resistance, $R_{\mathrm{ct}}$ represents the chargetransfer resistance through the SEI, and $C_{\mathrm{dl}}$ represents the capacitance of the electrical doublelayer. This type of system is common in the lithium-ion chemistry [1].

There are two conventional ways to display impedance data, Nyquist plots and Bode plots. In Nyquist plots, the real portion of the impedance is plotted on the $\mathrm{x}$-axis and the negative of the imaginary impedance is plotted on the y-axis (Figure 4-20). The negative of the imaginary impedance is used so that capacitance appears positive and inductance, not commonly seen in half cell impedance, appears negative. A projection of a Nyquist plot is shown in a lighter red dot color in the $3 \mathrm{D}$ plot of Figure 4-18. Nyquist plots are useful for visualizing

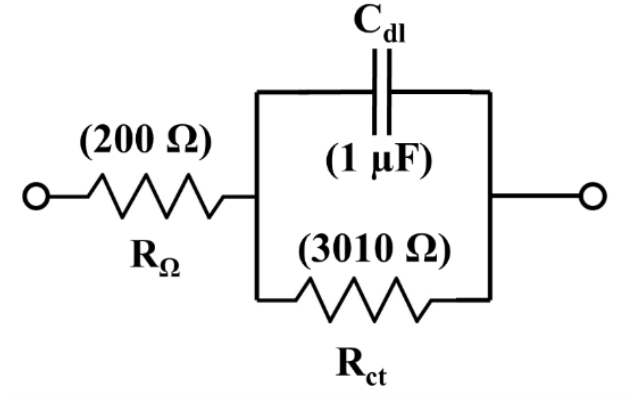

Figure 4-19: Electrical Circuit Schematic of Randles Cell 


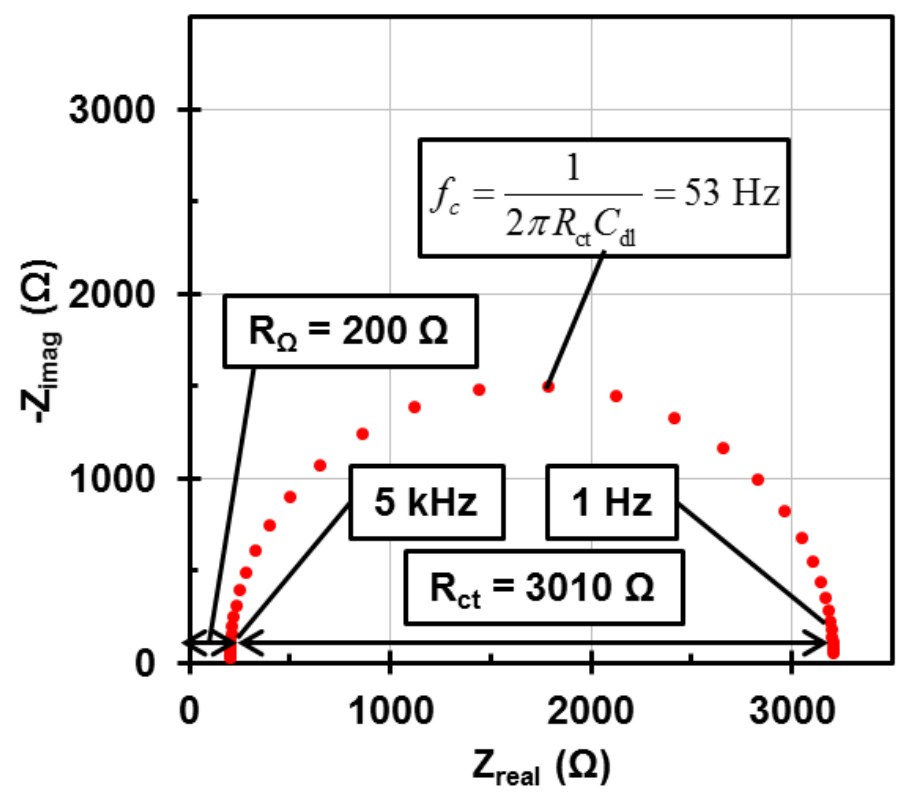

different characteristic time constants of an electrochemical cell; for a Randles cell, this appears in the impedance data as a single semicircle. Care should be taken to ensure that the Nyquist plot remains square with the $\mathrm{x}$ and y limits to ensure any semicircles present in the impedance data appear true and non-distorted. Nyquist plots

Figure 4-20: Nyquist Plot of Impedance Data from are also useful to visualize the series Randles Cell from $1 \mathrm{~Hz}-5 \mathrm{kHz}$

addition of resistances in an electrochemical cell. $R_{\Omega}$ is readily evident in a Nyquist plot as the resistance that the semicircle is offset by on the real impedance axis at high frequencies. Further, $R_{\mathrm{ct}}$ is the diameter of the impedance semicircle. At low frequencies, the impedance of the cell approaches the series addition of $R_{\Omega}$ and $R_{\mathrm{ct}}$. The double layer capacitance, $C_{\mathrm{dl}}$, can only be determined if the characteristic frequency, $f_{\mathrm{c}}$, of the RC circuit is known. The characteristic frequency relates directly to the $\mathrm{RC}$ time constant, $\tau$, the amount of time required to charge the capacitor to $63.2 \%$ in seconds by:

$$
\tau=R_{\mathrm{ct}} C_{\mathrm{dl}}=\frac{1}{2 \pi f_{\mathrm{c}}}
$$

Equation (4.29) is rearranged in Figure 4-20 to calculate the characteristic frequency of the RC circuit of the Randles Cell. In EIS experiments, the capacitance is unknown but can be readily determined by knowing the frequency used to generate the impedance corresponding to the $\mathrm{RC}$ time constant. 
Bode plots display the log of the modulus of the impedance on the primary y-axis and the negative of the phase angle on the secondary $y$-axis versus the $\log$ of the frequency domain of the experiment. A representative Bode plot for the Randles Cell is seen in Figure 4-21. The primary benefit of using Bode plots over Nyquist plots

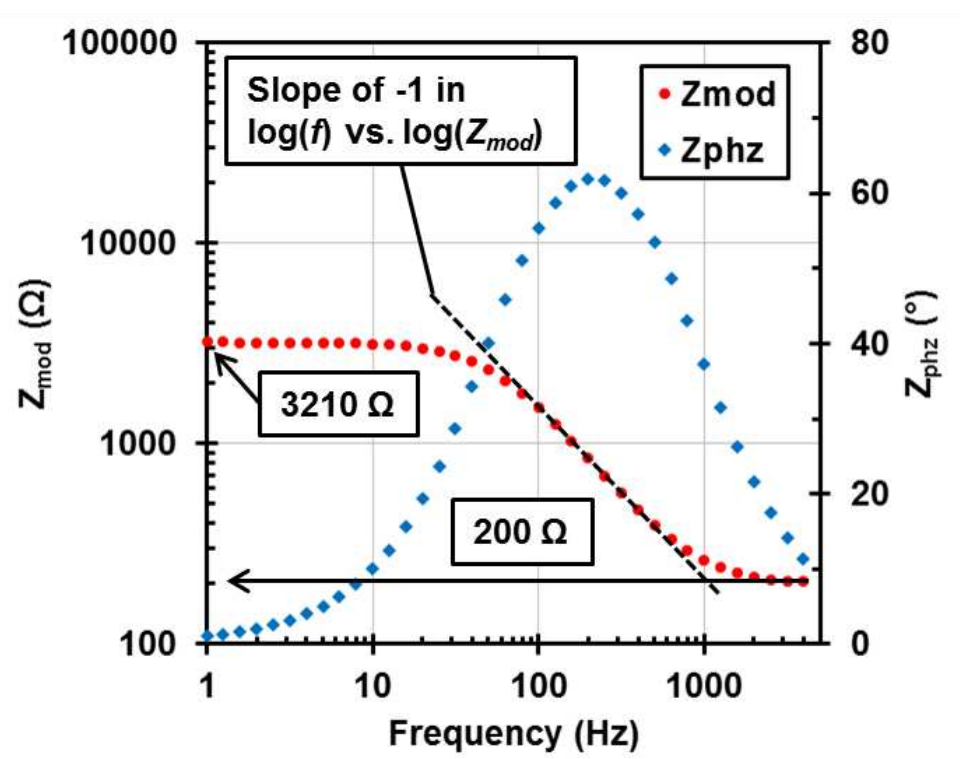

Figure 4-21: Bode Plot of Impedance Data from Randles Cell from $1 \mathrm{~Hz}-5 \mathrm{kHz}$

is the direct visualization of the frequency dependence of the modulus and the phase angle of impedance, which must be directly labeled on a Nyquist plot. The magnitude of $R_{\Omega}$ and the sum of $R_{\Omega}$ and $R_{\mathrm{ct}}$ can be visualized at the frequency limits of the test. In addition, the magnitude of the double-layer capacitance can be determined as the inverse of the intercept of the dashed line with a frequency of $0.16 \mathrm{~Hz}$. The dashed line shown in Figure 4-21 has a -1 slope in $\log (f)$ versus $\log \left(Z_{\text {mod }}\right)$ coordinates for intermediate frequencies when the impedance of the capacitor dominates the impedance of the cell, in this case approximately $100-500 \mathrm{~Hz}$. Within this frequency range, the total cell impedance corresponds to the impedance of the capacitor described by:

$$
Z(\omega)=\frac{1}{j \omega C_{\mathrm{dl}}}
$$

Therefore, the modulus of impedance of the cell within the log-log domain of the Bode plot within the intermediate frequencies of $100-500 \mathrm{~Hz}$ can be described by: 


$$
\log \left(Z_{\text {mod }}\right)=\log \left(\frac{1}{\omega C_{\mathrm{dl}}}\right)
$$

Note that the modulus of impedance, described by Equation (4.28), no longer requires complex representation which is why no complex notation is observed in Equation (4.31). Substituting Equation (4.14) into Equation (4.31) for $\omega$ and applying appropriate logarithm algebra results in the following:

$$
\log \left(Z_{\text {mod }}\right)=-\log (2 \pi f)-\log \left(C_{\mathrm{dl}}\right)
$$

At a frequency of $0.16 \mathrm{~Hz}$, the quantity of $2 \pi f$ equals 1, reducing Equation (4.32) to:

$$
Z_{\bmod (f=0.16 \mathrm{~Hz})}=\frac{1}{C_{\mathrm{dl}}}
$$

Note that the double-layer capacitance calculated in Equation (4.33) requires extrapolation of the linear fit of the intermediate frequency impedance to $0.16 \mathrm{~Hz}$, and the linear fit must be performed in a log-log domain.

The Nyquist and Bode plots of Figure 4-20 and Figure 4-21, respectively, can be modeled with electrical circuit elements that are directly analogous to the different components of an electrochemical cell. The complex impedance response for different electrical circuit elements is

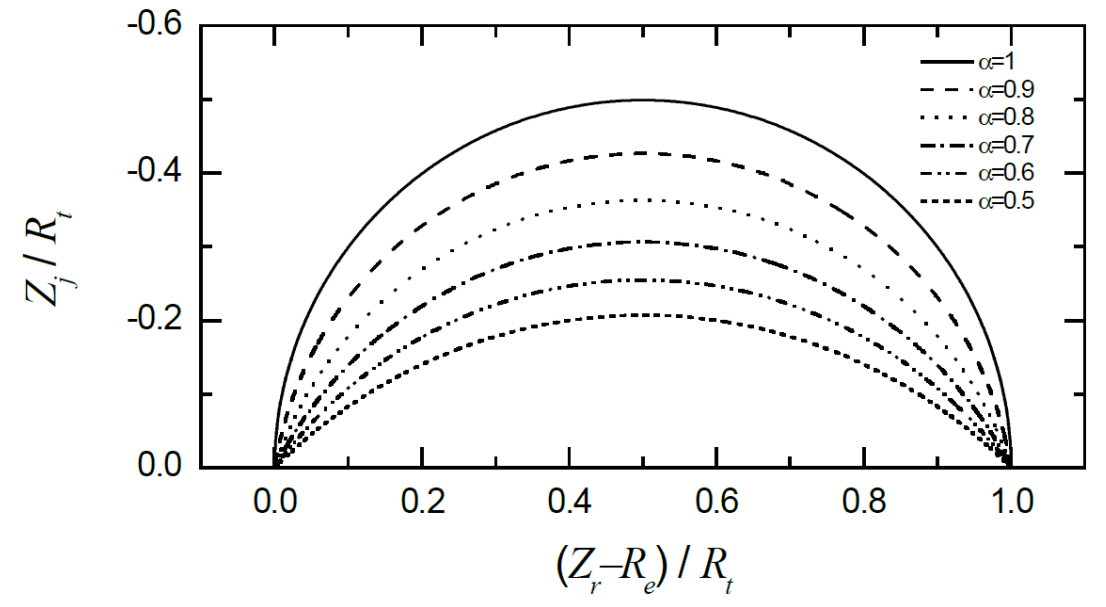

Figure 4-22: Nyquist Plot of Impedance of $\boldsymbol{R}$-CPE Circuit with Varying $\alpha$ [123] defined in Table 4-3. In particular, resistors and capacitors can be used to model the electronic and ionic conduction resistances of the cell, the electrochemical doublelayer, and the charge- 
transfer resistance of the SEI films on the surface of the electrodes. Two additional modeling elements worth noting are constant phase elements (CPE) and infinite Warburg diffusion elements. Although CPE and infinite Warburg diffusion elements do not have a direct electrical circuit analog, they are commonly used to interpret the impedance data of an

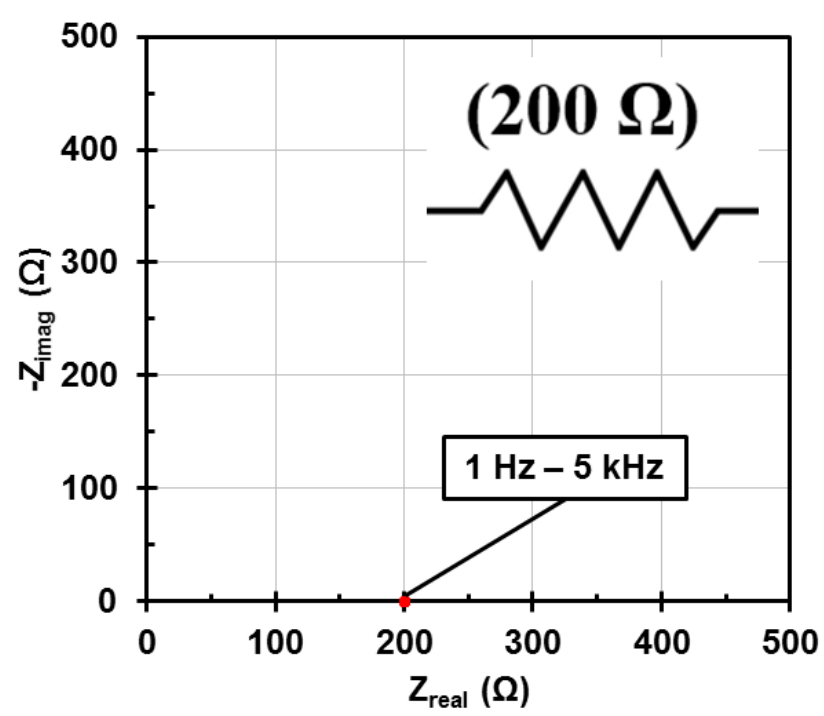

Figure 4-23: Nyquist Plot of $200 \Omega$ Resistor Impedance from $1 \mathrm{~Hz}-5 \mathrm{kHz}$

electrochemical cell. CPE model the capacitance of the electrochemical double-layer, but is able to adjust for its imperfect capacitor behavior due to varying surface distributions on the electrode surface [125]. Figure 4-22 shows the impedance response of a $R$-CPE circuit with varying levels of imperfect capacitor behavior as determined by $\alpha$. For a perfect capacitor, $\alpha$ equals 1 , and for imperfect capacitors $\alpha$ is less than 1. Infinite Warburg diffusion elements model the bulk mass transfer of the active material species into the electrode and only become active at low frequencies, which is the time scale required for mass transfer to occur.

Each of these circuit elements produce a unique impedance response that may be frequency-dependent. In the case of a resistor, the impedance response upon the application of an AC signal ranging from $1 \mathrm{~Hz}-5 \mathrm{kHz}$ is simply a real resistance corresponding to the resistance of the resistor (Figure 4-23). The impedance of a resistor is not frequency-dependent, which is why only a single point is observed in the Nyquist plot. 


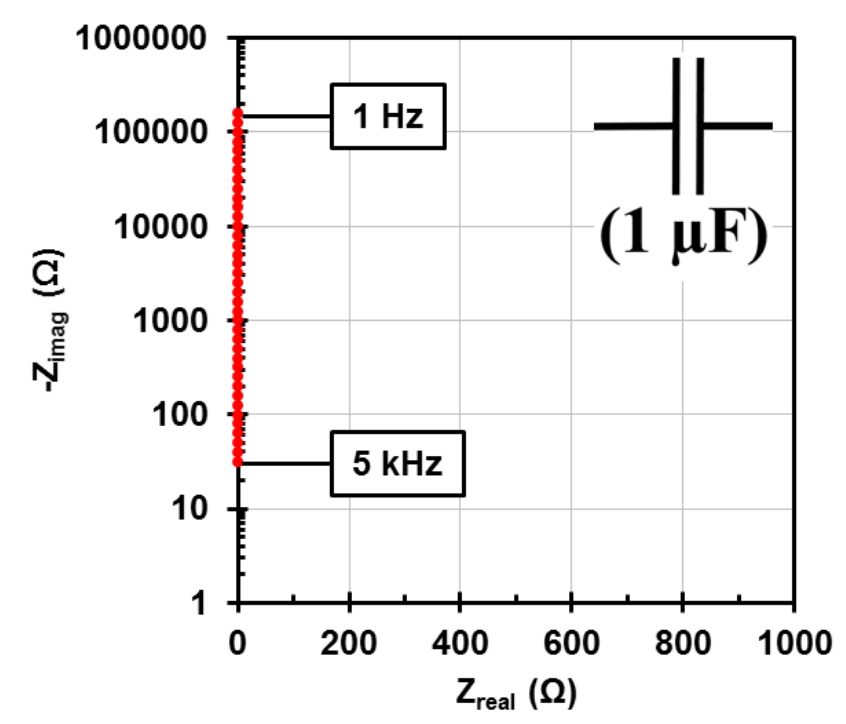

The impedance response of a capacitor is much different, and it only has impedance in imaginary space. In addition, the impedance of the capacitor is dependent upon frequency: at high frequencies the impedance approaches zero while at low frequencies the impedance approaches infinity (Figure 4-24).

Figure 4-24: Nyquist Plot of $1 \mu \mathrm{F}$ Capacitor Impedance from $1 \mathrm{~Hz}-5 \mathrm{kHz}$

Table 4-3: Impedance of Common Circuit Elements used for EIS Measurements

\begin{tabular}{llc} 
Circuit Element & Electrochemical Analogy & Impedance \\
\hline Resistor & $\begin{array}{l}\text { Electronic and ionic conduction resistances; } \\
\text { charge transfer resistance through SEI film on } \\
\text { electrode surface }\end{array}$ & $R$ \\
\hline Capacitor & Capacitance of the electrochemical double layer & $\frac{1}{j \omega C}$ \\
\hline $\begin{array}{l}\text { Constant Phase } \\
\text { Element }\end{array}$ & $\begin{array}{l}\text { Imperfect capacitance of the electrochemical } \\
\text { double layer }\end{array}$ & $\frac{1}{Q(j \omega)^{\alpha}}$ \\
\hline $\begin{array}{l}\text { Infinite Warburg } \\
\text { Impedance }\end{array}$ & $\begin{array}{l}\text { Mass diffusion of active material species } \\
\text { through the electrode-electrolyte interface }\end{array}$ & $\frac{1}{W_{\mathrm{d}}(j \omega)^{1 / 2}}$ \\
\hline Inductor & Reactant adsorption, test setup errors & $j \omega L$
\end{tabular}

The impedance of the resistor and capacitor individually do not relate to the impedance spectra of a Randles Cell. However, these electrical elements can be combined to create an equivalent circuit to model the impedance (Figure 4-19). The equivalent impedance of the circuit can be analytically determined by using the following two formulas for $n$ elements in series (4.34) or parallel (4.35): 


$$
\begin{gathered}
Z_{e q}=Z_{1}+Z_{2}+Z_{3}+\cdots+Z_{n} \\
\frac{1}{Z_{e q}}=\frac{1}{Z_{1}}+\frac{1}{Z_{2}}+\frac{1}{Z_{3}}+\cdots+\frac{1}{Z_{n}}
\end{gathered}
$$

For the Randles Cell, there are three circuit elements that produce impedance: $R_{\Omega}, R_{\mathrm{ct}}$, and $C_{\mathrm{dl}}$. The impedance of these respective components individually is:

$$
\begin{gathered}
Z_{1}=R_{\Omega} \\
Z_{2}=R_{\mathrm{ct}} \\
Z_{3}=\frac{1}{j \omega C}
\end{gathered}
$$

Figure 4-19 shows that $R_{\mathrm{ct}}$ and $C_{\mathrm{dl}}$ are connected in parallel and this parallel connection is in series with $R_{\Omega}$. To determine the equivalent impedance of the Randles Cell, the equivalent impedance of the $R_{\mathrm{ct}}$ and $C_{\mathrm{dl}}$ parallel connection must first be determined. Then, the RC equivalent impedance can be combined with the series impedance of $R_{\Omega}$ to calculate the equivalent impedance of the Randles Cell. Applying Equation (4.35) to combine the parallel connection of the $R_{\mathrm{ct}}$ and $C_{\mathrm{dl}}$ elements yields:

$$
\frac{1}{Z_{23}}=\frac{1}{R_{\mathrm{ct}}}+\frac{1}{j \omega C_{\mathrm{dl}}}
$$

Further simplifying Equation (4.39) with complex algebra gives:

$$
Z_{23}=\frac{\frac{R_{\mathrm{ct}}}{\omega^{2} C_{\mathrm{dl}}{ }^{2}}-\frac{j R_{\mathrm{ct}}{ }^{2}}{\omega C_{\mathrm{dl}}}}{R_{\mathrm{ct}}{ }^{2}+\frac{1}{\omega^{2} C_{\mathrm{dl}}{ }^{2}}}
$$

The equivalent impedance of the RC circuit, $Z_{23}$, can now be combined with the series impedance of $R_{\Omega}$ using Equation (4.34) to determine the total equivalent impedance of the Randles Cell: 


$$
Z_{\mathrm{eq}}=R_{\Omega}+\frac{\frac{R_{\mathrm{ct}}}{\omega^{2} C_{\mathrm{dl}}{ }^{2}}-\frac{j R_{\mathrm{ct}}{ }^{2}}{\omega C_{\mathrm{dl}}}}{R_{\mathrm{ct}}{ }^{2}+\frac{1}{\omega^{2} C_{\mathrm{dl}}{ }^{2}}}
$$

The total impedance described in Equation (4.41) is in complex rectangular notation, useful for separating the real and imaginary portions of impedance for creating a Nyquist plot. Moreover, the total impedance is only a function of angular frequency, $\omega ; R_{\Omega}, R_{\mathrm{ct}}$, and $C_{\mathrm{dl}}$ all represent the magnitude of resistance or capacitance of the circuit elements.

In the provided Randles Cell example, the magnitude of the equivalent circuit elements is known. However, in an EIS experiment on a half cell, the magnitude of resistance and capacitance of the circuit elements is unknown. To determine these parameters, a non-linear least squares fitting (NLLS) algorithm contained within the Gamry Echem Analyst software is used. The general frequency-dependent equation of impedance is input into the program. The experimenter provides initial estimates of each of the circuit element parameters and the algorithm iterates through parameter adjustments to minimize the error of the fit. Initial estimates of the parameters can be made from Nyquist and Bode plots of the measured impedance data using the aforementioned techniques. After the fit converges on an acceptable solution, the final parameters for each of the elements are determined. This NLLS process is performed using a built-in Simplex Method algorithm to update parameter guesses in the software.

Tracking the changes of these parameters in the impedance data provides significant insight into the changes of the electrochemical cell under study. In a study that utilizes a Randles Cell equivalent circuit, a growth in the cell resistance, $R_{\Omega}$, would indicate loss of electrical contact within working electrode or precipitation of the active species in the electrolyte. Furthermore, a loss in the double layer capacitance, $C_{\mathrm{dl}}$, would indicate that less active material surface area on the working electrode exists. In addition, an increase in the charge-transfer resistance, $R_{\mathrm{ct}}$, would 
indicate that a thicker SEI layer has formed. Tracking these model parameters and their changes over the life of an electrochemical cell can provide valuable insight into what portions of the cell should be targeted for improvement.

\subsubsection{Equivalent Circuit Model Used for Half and Full Cell Testing}

The equivalent circuit used for modeling the impedance data measured from half and full cells is shown in Figure 4-25. This equivalent circuit is chosen for its simplicity in

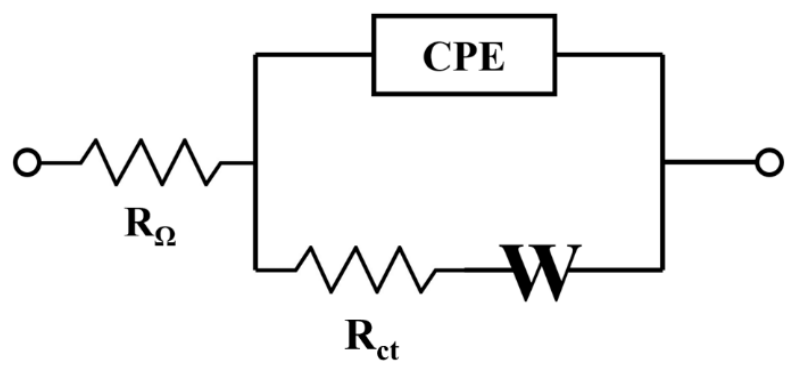
modeling the observed impedance spectra Figure 4-25: Equivalent Circuit Model Used which contained only one time constant and Tests for Impedance Fitting in Half and Full Cell showed diffusion controlled impedance at low frequencies. The measured impedance data is very similar to that collected by Arai on HFE-7100-containing electrolytes, who only fit the semicircle portion of the impedance, ignoring the diffusion control at low frequencies [86]. The equivalent circuit in Figure 4-25 shares many similarities with the previously discussed Randles Cell. In place

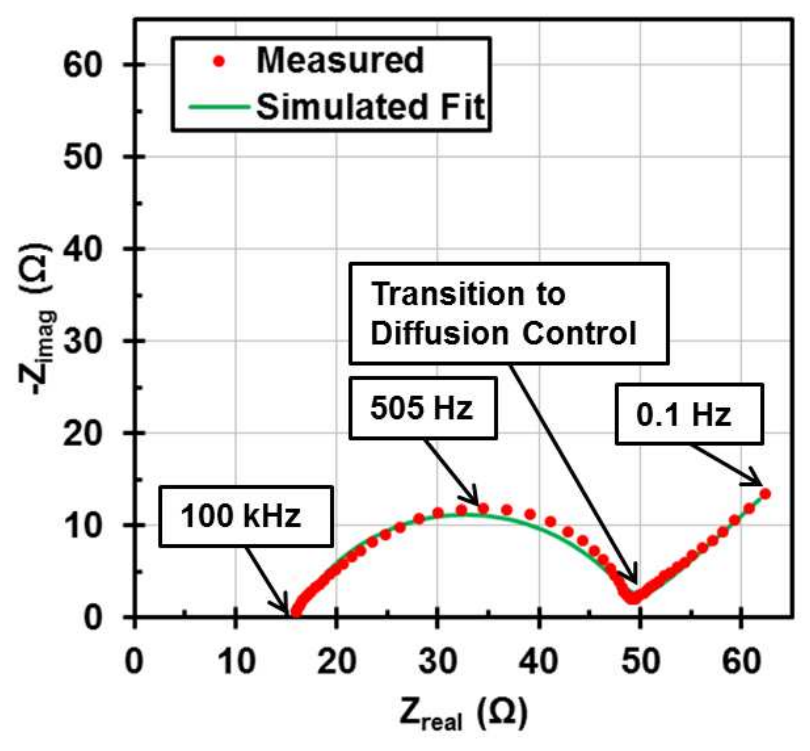

of the capacitor, a constant phase element (CPE) is used. The impedance measurements made on the half and full cells were much more accurately modeled with a CPE due to its ability to accommodate the varying surface distributions of the active materials, which produced a non-ideal capacitive response. The diffusion

Figure 4-26: Example of Impedance Model Fitting to Measured Data for $\mathrm{Cu}_{2} \mathrm{Sb} / \mathrm{Li}$ Cell at $0.5 \mathrm{~V}$ vs. $\mathrm{Li} / \mathrm{Li}^{+}$from $0.1 \mathrm{~Hz}-100 \mathrm{kHz}$ 
controlled portion of the impedance at low frequencies was modeled with an infinite Warburg diffusion element.

An example of the measured impedance spectra with its equivalent model fit of a $\mathrm{Cu}_{2} \mathrm{Sb}$ half cell after 20 cycles with

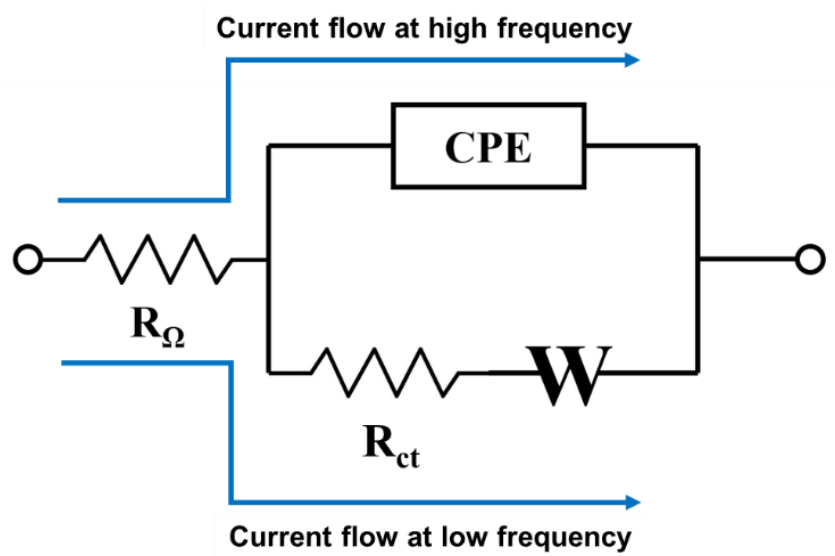
the cell held at $0.5 \mathrm{~V}$ vs. $\mathrm{Li} / \mathrm{Li}^{+}$is shown in

Figure 4-27: Frequency-Dependent Preferential Current Paths Through Equivalent Circuit

Figure 4-26. There is clearly one RC time constant (semicircle) present in the impedance data and a transition to diffusion controlled impedance at low frequencies. An infinite Warburg diffusion element is used to model the diffusion controlled impedance, which appears as a diagonal line on a Nyquist plot with a slope of 0.5 . Although the diffusion controlled impedance is observed and modeled, the analysis of impedance data focuses primarily on the $R_{\mathrm{ct}}, \mathrm{CPE}$, and $R_{\Omega}$ elements, which provide direct insight into the electrode-electrolyte interface, especially the SEI. The characteristic frequency of the $R_{\mathrm{ct}}-\mathrm{CPE}$ circuit is calculated using the following formula provided by Orazem et al. [126]:

$$
f_{\mathrm{c}}=\frac{1}{2 \pi R_{\mathrm{ct}} Q^{1 / \alpha} R_{\mathrm{ct}}^{(1-\alpha) / \alpha}}
$$

Figure 4-27 shows the preferential current paths through the equivalent circuit model, which change depending upon the applied frequency of the potential signal. At high frequencies, the impedance of the CPE approaches zero, leading to a preferential current path through the top portion of the $R_{\mathrm{ct}} \mathrm{CPE}$ circuit. This is why at high frequencies, the equivalent impedance of the cell is reduced to $R_{\Omega}$. Conversely, at low frequencies when the CPE has sufficient time to accumulate charge, the impedance of the CPE approaches infinity creating a preferential current 
path through the lower portion of the $R_{\mathrm{ct}} \mathrm{CPE}$ circuit. At even lower frequencies, the impedance of the infinite Warburg diffusion element grows adding to the series resistance of $R_{\Omega}$ and $R_{\mathrm{ct}}$.

\subsubsection{Test Parameters and Equipment Used}

The equipment required for galvanostatic cycling and electrochemical impedance spectroscopy of half cells was extensive. The following sections detail the equipment used to manufacture the LFP, LTO, and $\mathrm{Cu}_{2} \mathrm{Sb}$ electrodes. Thereafter, the equipment used for the galvanostatic cycling and electrochemical impedance spectroscopy measurements are described.

4.4.4.1. Fabrication of Lithium Iron Phosphate and Lithium Titanate Oxide Slurry-Based Electrodes

The LFP and LTO electrodes used in this work were manufactured on electrode coating and calendaring equipment at Prieto Battery. The equipment used included a weight scale, paint can shaker, draw down machine with doctor blade, heat lamp, vacuum oven, and calendaring machine. To create these electrodes, a slurry containing the active material particles was mixed and thoroughly homogenized. The slurry was then uniformly coated onto a prepared aluminum or copper foil for the LFP or LTO electrodes, respectively. The freshly coated foil was then baked under a heat lamp for $10-15$ minutes and then transported to a vacuum oven held at $120^{\circ} \mathrm{C}$ and baked overnight to remove all the liquid solvent used to suspend the homogenized slurry. The dried electrode was then calendared to its final thickness using a calendaring machine. A more detailed slurry-based electrode coating procedure with pictures is provided in Appendix C.

Table 4-4 lists the components of the slurry for the LFP and LTO electrodes. The active material comprises the majority of the electrode slurry mixture by weight. In addition, two different types of graphite, Timcal KS6 and C65, are used to improve the electrical contact between the active material particles and reduce the electronic resistance of the electrode. Furthermore, two 
different types of PVDF binder are used to improve the particle-particle binding (Kynar 761) and the particle-foil binding (Kynar 161). The LTO electrodes required additional Kynar 761 binder to improve the adhesion of the electrode coating. (The initial LTO slurry mixture mirroring the LFP slurry mixture did not produce successful coatings.) N-Methyl-2-pyrrolidone (NMP) was the solvent used to homogeneously suspend the electrode slurry purchased from Sigma Aldrich.

Table 4-4: Slurry Composition for LFP and LTO Electrodes by Weight Percent Phostech,

\begin{tabular}{llllll} 
Supplier & Aldrich & Timcal & Timcal & Kynar & Kynar \\
\hline & $\begin{array}{l}\text { Active } \\
\text { Material } \\
(\boldsymbol{\%})\end{array}$ & $\begin{array}{l}\text { KS6 } \\
\text { Graphite } \\
(\boldsymbol{\%})\end{array}$ & $\begin{array}{l}\text { C65 } \\
\text { Graphite } \\
(\boldsymbol{\%})\end{array}$ & $\begin{array}{l}\text { 161 PVDF } \\
\text { Binder } \\
(\%)\end{array}$ & $\begin{array}{l}\text { 761 PVDF } \\
\text { Binder } \\
(\%)\end{array}$ \\
\hline $\begin{array}{l}\text { Electrode } \\
(\mathrm{LiFePO})\end{array}$ & 77.50 & 9.75 & 5.25 & 1.13 & 6.38 \\
\hline $\begin{array}{l}\text { Lithium Titanate Oxide } \\
\left(\mathrm{Li}_{4} \mathrm{Ti}_{5} \mathrm{O}_{12}\right)\end{array}$ & 74.33 & 9.35 & 5.04 & 1.69 & 9.59
\end{tabular}

For LFP electrodes, the slurry was coated onto $25 \mu \mathrm{m}$ aluminum foil and calendared to a final thickness of approximately $100 \mu \mathrm{m}$ with an average loading of $7.3 \mathrm{mg} \mathrm{cm}{ }^{-2}$. For LTO electrodes, the slurry was coated onto a $15 \mu \mathrm{m}$ copper foil and calendared to a final thickness of approximately $55 \mu \mathrm{m}$ with an average loading of $4.1 \mathrm{mg} \mathrm{cm}^{-2}$. After vacuum drying, the electrodes were punched into $19 \mathrm{~mm}$ disks and pumped into the argon glove box for assembly in half cells.

4.4.4.2. Fabrication of Copper Antimonide Electrodes

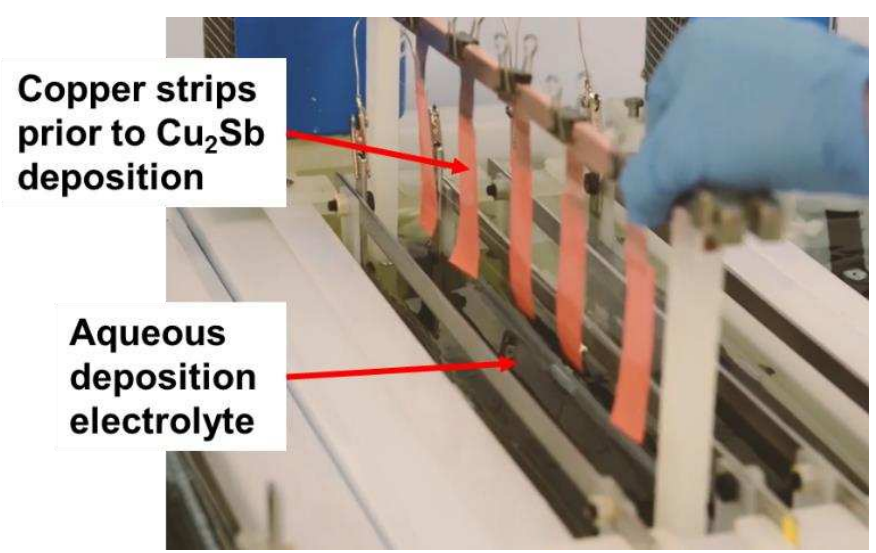

Figure 4-28: $\mathrm{Cu}_{2} \mathrm{Sb}$ Electrodeposition Setup at Prieto Battery
The $\mathrm{Cu}_{2} \mathrm{Sb}$ electrodes were produced by room temperature aqueous electrodeposition onto a $15 \mu \mathrm{m}$ copper foil substrate by a process described elsewhere [127]. Figure 4-28 shows the $\mathrm{Cu}_{2} \mathrm{Sb}$ electrodeposition setup located at Prieto Battery, which was used for the 
manufacturing of the $\mathrm{Cu}_{2} \mathrm{Sb}$ electrodes in this study. A picture of the completed $\mathrm{Cu}_{2} \mathrm{Sb}$ electrodeposition onto the copper foil is seen in Figure 4-29. The benefits of electrodeposition as a coating technique include

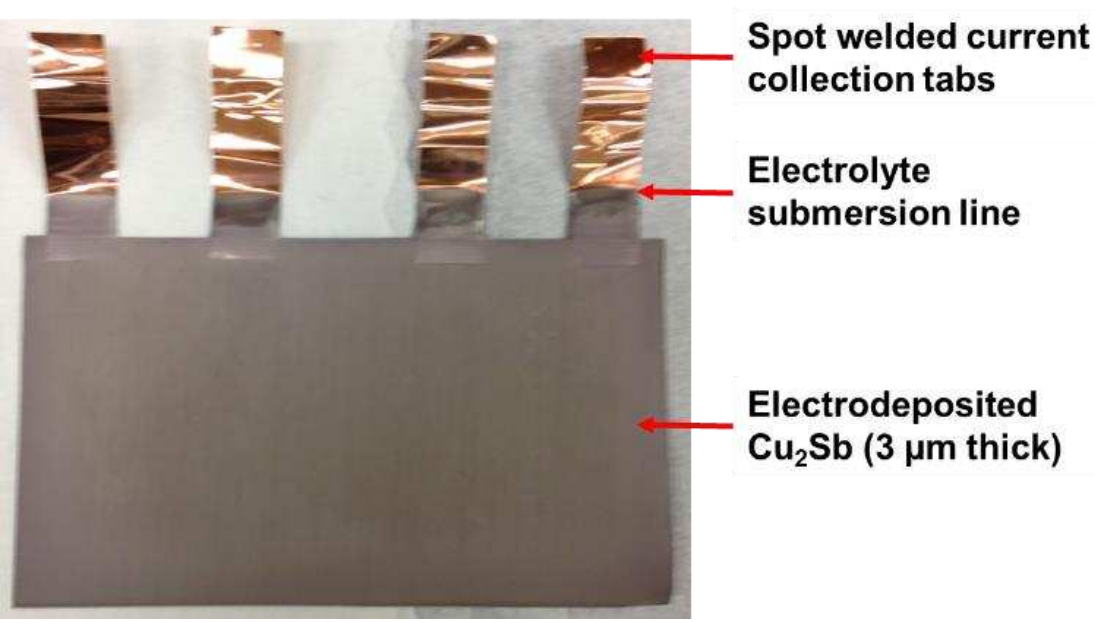

Figure 4-29: Post-Electrodeposition of $3 \mu \mathrm{m}$ of $\mathrm{Cu}_{2} \mathrm{Sb}$ onto 15 $\mu \mathrm{m}$ Copper Foil

good electrical contact of the $\mathrm{Cu}_{2} \mathrm{Sb}$ coating with the foil substrate without the use of PVDF binding agents or carbon conductive additives. For electrodeposited $\mathrm{Cu}_{2} \mathrm{Sb}$ electrodes, $100 \%$ of the coating mass was active material whereas only $77.5 \%$ and $74.33 \%$ of the mass was active material for LFP and LTO electrodes respectively. The $\mathrm{Cu}_{2} \mathrm{Sb}$ coating thickness was approximately $3 \mu \mathrm{m}$ with an average loading of $2.1 \mathrm{mg} \mathrm{cm}^{-2}$. The $\mathrm{Cu}_{2} \mathrm{Sb}$-coated copper foil was then punched into $19 \mathrm{~mm}$ disks and pumped into the argon glove box for half cell assembly.

\subsubsection{Galvanostatic Cycling Voltage Limits and Determination of Cycling Current}

Each cell combination was cycled on an Arbin BT-2143 battery tester at an approximate $0.5 \mathrm{C}$ rate in 2-10 cycle increments for a total of 20 cycles in a Tenney environmental chamber held

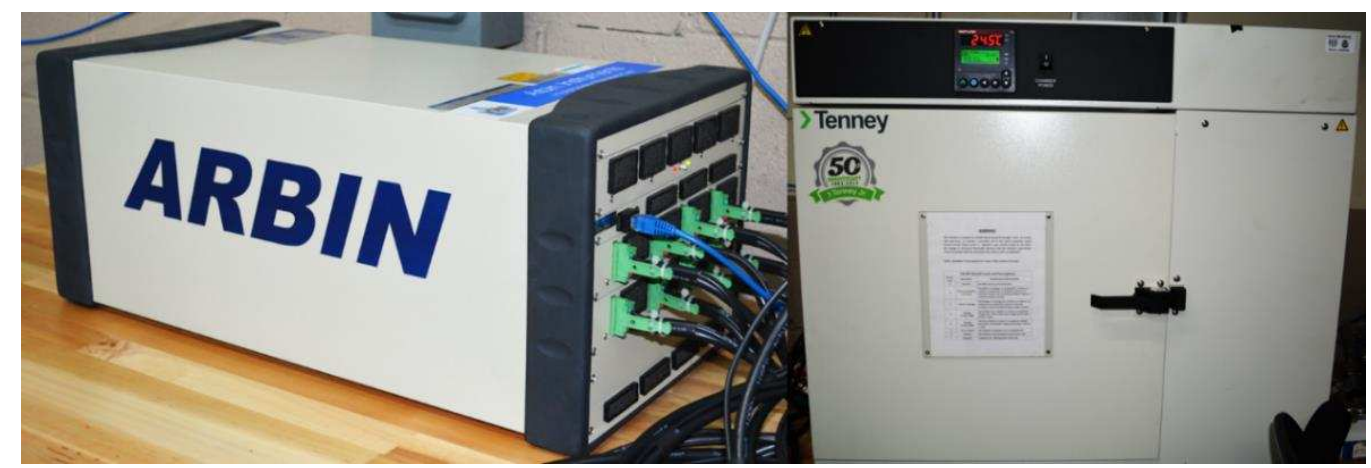

Figure 4-30: Arbin BT-2143 Battery Tester (Left) and Tenney Environmental Chamber (Right) 
at $25^{\circ} \mathrm{C}$ (Figure 4-30). The Arbin contains 8 independent channels with $0-5 \mathrm{~V} \pm 1 \mathrm{mV}$ voltage control and measurement. Each channel contains three current ranges $500 \mathrm{~mA} / 10 \mathrm{~mA} / 100 \mu \mathrm{A}$ $\pm 0.02 \%$ full scale range accuracy for both control and measurement. The Tenney environmental chamber contains a sealable $7.62 \mathrm{~cm}$ access port for the Arbin test leads. The half cells were electrically connected to the Arbin test leads as shown in Figure 4-31. The Arbin battery tester uses four-point Kelvin probe connections for all channels. The white and green leads are for positive and negative terminal voltage sense, respectively. The red and black leads are currentcarrying wires for the positive and negative terminals respectively. The Arbin leads are terminated with alligator clips, which are directly attached to the 10-32 terminal screws on the half and full cells.

The current applied and cell voltage limits for each half cell combination varied: $386 \mu \mathrm{A}$ $\mathrm{cm}^{-2}$ for LFP/Li cells between 2.5 and $4 \mathrm{~V}$ vs. $\mathrm{Li} / \mathrm{Li}^{+} ; 386 \mu \mathrm{A} \mathrm{cm}{ }^{-2}$ for LTO/Li cells between 1 and $2 \mathrm{~V}$ vs. $\mathrm{Li} / \mathrm{Li}^{+}$; and $87.7 \mu \mathrm{A} \mathrm{cm}{ }^{-2}$ for $\mathrm{Cu}_{2} \mathrm{Sb} / \mathrm{Li}$ cells between 0.5 and $0.95 \mathrm{~V}$ vs. $\mathrm{Li} / \mathrm{Li}^{+}$. In addition, the $\mathrm{Cu}_{2} \mathrm{Sb} / \mathrm{Li}$ cells were cycled at an approximate $0.1 \mathrm{C}$ rate for the first lithiation, $17.54 \mu \mathrm{A} \mathrm{cm}{ }^{-2}$. After completion of the first lithiation, the $\mathrm{Cu}_{2} \mathrm{Sb} / \mathrm{Li}$ cells were cycled using the previously specified $0.5 \mathrm{C}$ rate.

The applied current was initially determined by calculating the expected capacity of the half cell, $C_{\text {cell. }}$ This was calculated by the mass of the active material present in the working electrode multiplied by the theoretical capacity, $C_{\text {Theo, }}$ of the active material (Table 4-5):

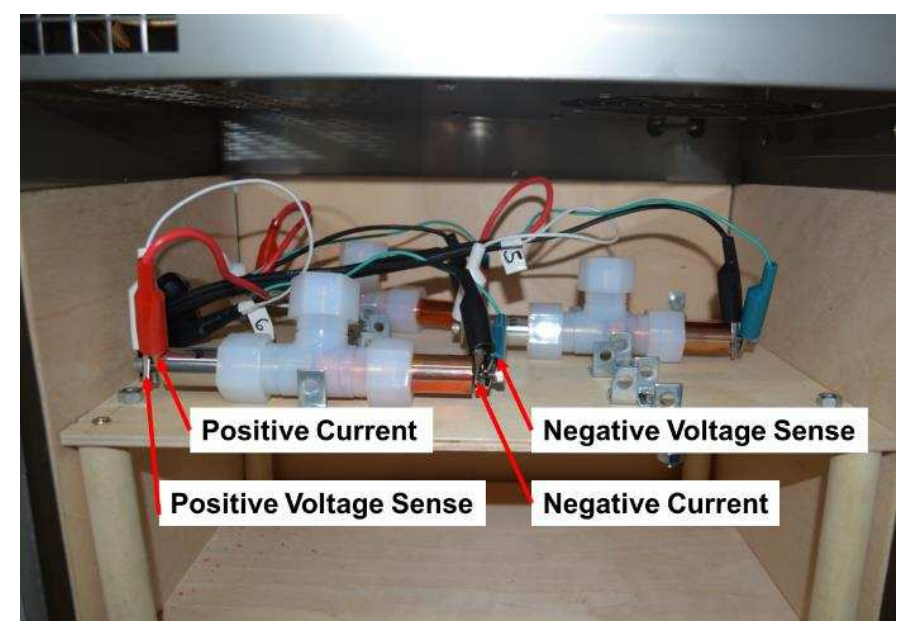

Figure 4-31: Arbin Battery Tester Lead Connections to Full Cells in Environmental Chamber 


$$
C_{\text {cell }}=\left(m_{\text {coat }} Y_{\mathrm{AM}}\right) \cdot C_{\text {Theo }}
$$

Where $m_{\text {coat }}$ is the mass of the electrode coating and $Y_{\mathrm{AM}}$ is the mass fraction of active material, used only for the slurry-based LFP and LTO electrodes. To approximate the current required for a $0.5 \mathrm{C}$ rate, the expected capacity was divided accordingly:

$$
I_{0.5 C}=\frac{C_{\text {Theo }}}{2}
$$

The 2 in Equation (4.44) has units of hours: one lithiation or delithiation of the active material will be completed in 2 hours at a $0.5 \mathrm{C}$ rate. The resulting current, $I_{0.5 \mathrm{C}}$, was applied to the half cells and adjusted accordingly to best approximate a $0.5 \mathrm{C}$ rate.

The voltage limits used for the half cells was determined by the operation potential of the active materials. LFP provides its useful capacity at a constant voltage of approximately $3.5 \mathrm{~V}$ vs. $\mathrm{Li} / \mathrm{Li}^{+}$; therefore, the voltage limits were extended around this value to maximize the capacity utilization of the active material $\left(2.5-4 \mathrm{~V}\right.$ vs. $\left.\mathrm{Li} / \mathrm{Li}^{+}\right)$. Similarly, the lower and upper voltage limits for LTO half cells were 1 and $2 \mathrm{~V}$, respectively, which is $0.5 \mathrm{~V}$ above and below the constant potential it delivers useful capacity at low rates. The potential limits used for $\mathrm{Cu}_{2} \mathrm{Sb}$ were selected to best utilize the most reversible two-phase reaction of the active material, which occurs between 0.5 and $0.95 \mathrm{~V}$ vs. $\mathrm{Li} / \mathrm{Li}^{+}[128]$. The selection of voltage limits directly affects the capacity of the cell, especially for $\mathrm{Cu}_{2} \mathrm{Sb}$. Table 4-5 shows the interaction of the cell voltage limits with the theoretical and cycling capacity. The cycling capacity was determined as an average measured capacity of the half cells studied for both the baseline and MFE to provide insight into the influence of voltage limits on the measured capacity. For LFP and LTO, the cycling capacity and the theoretical capacity are close. The differences between these two values are a result of the capacity loss associated with SEI formation on the active material surface during the first few cycles. 
Table 4-5: Half Cell Voltage Limits and Capacity

\begin{tabular}{|c|c|c|c|c|c|}
\hline Active Material & 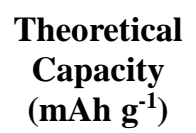 & $\begin{array}{l}\text { Density } \\
\left(\mathrm{g} \mathrm{cm}^{-3}\right)\end{array}$ & $\begin{array}{c}\text { Upper Voltage } \\
\text { Limit } \\
\left(\text { V vs. } \mathbf{L i} / \mathbf{L i}^{+}\right)\end{array}$ & $\begin{array}{c}\text { Lower } \\
\text { Voltage Limit } \\
\left(\text { V vs. } \mathbf{L i} / \mathbf{L i}^{+}\right)\end{array}$ & $\begin{array}{c}\text { Cycling } \\
\text { Capacity } \\
\left(\mathbf{m A h} \mathbf{g}^{-1}\right)\end{array}$ \\
\hline Lithium Iron Phosphate $\left(\mathrm{LiFePO}_{4}\right)$ & 170 & 3.601 & 4.0 & 2.5 & 145 \\
\hline Copper Antimonide $\left(\mathrm{Cu}_{2} \mathrm{Sb}\right)$ & 323 & 8.510 & 0.95 & 0.5 & 85 \\
\hline Lithium Titanate Oxide $\left(\mathrm{Li}_{4} \mathrm{Ti}_{5} \mathrm{O}_{12}\right)$ & 175 & 3.429 & 2.0 & 1.0 & 150 \\
\hline
\end{tabular}

The test schedule was defined in Arbin MITS PRO software (version 4.32). The schedule file defines each step type of the galvanostatic cycling experiment, data sampling rate, and manipulation of internal variables. Table 4-6 defines the generic test schedule created in the MITS PRO software used for galvanostatic cycling experiments. The only exception to the provided test schedule is for the $\mathrm{Cu}_{2} \mathrm{Sb} / \mathrm{Li}$ cells which use a reduced (0.1C) current for the first lithiation.

Table 4-6: Generic Half Cell Test Schedule for Galvanostatic Cycling Tests

\begin{tabular}{|c|c|c|c|c|c|}
\hline $\begin{array}{l}\text { Step } \\
\text { Number }\end{array}$ & Description & $\begin{array}{l}\text { Control } \\
\text { Type }\end{array}$ & $\begin{array}{l}\text { Control } \\
\text { Value }\end{array}$ & Step Limits & $\begin{array}{l}\text { Data } \\
\text { Sampling } \\
\text { Rate }\end{array}$ \\
\hline 1 & Rest & N/A & Time $(\mathrm{t})$ & $\mathrm{t} \geq 30$ minutes & $0.1 \mathrm{~Hz}$ \\
\hline 2 & $\begin{array}{l}\text { Delithiate (LFP) } \\
\left.\text { Lithiate (LTO, } \mathrm{Cu}_{2} \mathrm{Sb}\right)\end{array}$ & Current & $\begin{array}{l}0.5 \mathrm{C} \\
\text { Current }\end{array}$ & $\begin{array}{l}\text { Voltage }(\mathrm{V}) \geq \\
\text { UVL (LFP) or } \\
\mathrm{V} \leq \mathrm{LVL}(\mathrm{LTO}, \\
\left.\mathrm{Cu}_{2} \mathrm{Sb}\right)\end{array}$ & $0.1 \mathrm{~Hz}$ \\
\hline 3 & Rest & N/A & Time $(\mathrm{t})$ & $\mathrm{t} \geq 2$ minutes & $0.1 \mathrm{~Hz}$ \\
\hline 4 & $\begin{array}{l}\text { Lithiate (LFP) } \\
\text { Delithiate (LTO, } \\
\left.\mathrm{Cu}_{2} \mathrm{Sb}\right)\end{array}$ & Current & $\begin{array}{l}0.5 \mathrm{C} \\
\text { Current }\end{array}$ & $\begin{array}{l}\mathrm{V} \leq \mathrm{LVL}(\mathrm{LFP}) \\
\text { or } \\
\mathrm{V} \geq \mathrm{UVL}(\mathrm{LTO}, \\
\left.\mathrm{Cu}_{2} \mathrm{Sb}\right)\end{array}$ & $0.1 \mathrm{~Hz}$ \\
\hline 5 & Rest & N/A & Time (t) & $\mathrm{T} \geq 2$ minutes & $0.1 \mathrm{~Hz}$ \\
\hline 6 & $\begin{array}{l}\text { Increment cycle } \\
\text { index, reset } \\
\text { charge/discharge } \\
\text { capacity and energy }\end{array}$ & Software & N/A & N/A & N/A \\
\hline 7 & 10 cycle loop & N/A & $\begin{array}{l}\text { Cycle } \\
\text { Index } \\
(\mathrm{CI})\end{array}$ & $\begin{array}{l}\text { If } \mathrm{CI} \leq 10 \text {, return } \\
\text { to Step } 2 \text {, else } \\
\text { Step } 8\end{array}$ & N/A \\
\hline 8 & Final rest & N/A & Time (t) & $\mathrm{t} \geq 1$ hour & $0.1 \mathrm{~Hz}$ \\
\hline
\end{tabular}




\subsubsection{Electrochemical Impedance Spectroscopy Test Parameters}

The EIS experiments were performed with a Gamry Reference 3000 potentiostat, the same instrument used for cyclic voltammetry experiments (Figure 4-8). After 10 and 20 lithiationdelithiation cycles, each cell underwent potentiostatic electrochemical impedance spectroscopy (EIS) at the upper and lower voltage limits (UVL and LVL respectively). A $5 \mathrm{mV}_{\text {rms }}$ signal was applied between the frequencies $0.1 \mathrm{~Hz}$ and $100 \mathrm{kHz}$ with 10 points per decade of frequency. The resulting impedance data was used to compare the interfacial impedance of the baseline and candidate electrolyte in each cell combination. Gamry Framework software was used to define all the aforementioned test parameters using the Sequence Wizard. To ensure the potential of the cell under test was at its potentiostatic test condition prior to the EIS experiment, a constant-current lithiation or delithiation step was added prior to the EIS experiment at the UVL and LVL. The experimental sequence is described in Table 4-7.

\begin{tabular}{|c|c|c|c|}
\hline $\begin{array}{l}\text { Step } \\
\text { Number }\end{array}$ & $\begin{array}{l}\text { Experiment } \\
\text { Type }\end{array}$ & Description & Data Sampling Rate \\
\hline 1 & Read Voltage & $\begin{array}{l}\text { Cell voltage is read to ensure leads } \\
\text { are correctly connected to cell }\end{array}$ & $1 \mathrm{~Hz}$ \\
\hline 2 & Charge & $\begin{array}{l}\text { Cell is charged with constant } 0.5 \mathrm{C} \\
\text { current until the UVL of the cell is } \\
\text { reached }\end{array}$ & $0.2 \mathrm{~Hz}$ \\
\hline 3 & $\begin{array}{l}\text { Potentiostatic } \\
\text { EIS }\end{array}$ & $\begin{array}{l}\text { Cell undergoes EIS experiment at } \\
\text { UVL }\end{array}$ & $\begin{array}{l}10 \text { points per decade } \\
\text { of frequency }\end{array}$ \\
\hline 4 & Discharge & $\begin{array}{l}\text { Cell is discharged with constant } \\
0.5 \mathrm{C} \text { current until the LVL of the } \\
\text { cell is reached }\end{array}$ & $0.2 \mathrm{~Hz}$ \\
\hline 5 & $\begin{array}{l}\text { Potentiostatic } \\
\text { EIS }\end{array}$ & $\begin{array}{l}\text { Cell undergoes EIS experiment at } \\
\text { LVL }\end{array}$ & $\begin{array}{l}10 \text { points per decade } \\
\text { of frequency }\end{array}$ \\
\hline
\end{tabular}

Gamry Echem Analyst software is used to perform equivalent circuit fitting of the impedance data to extract model parameters. The Simplex Method, a built-in algorithm, is used to vary model parameters to determine the best model fit. The reported impedance data for model 
parameters are an average of three cells for each electrolyte, while the impedance data shown in Nyquist plots is of a single cell representative of the average.

\subsubsection{Results and Discussion}

The results and discussion of the galvanostatic cycling and impedance spectroscopy tests are presented in the following sections. For the lithiation and delithiation capacity and coulombic efficiency plots, the reported data is an average of a minimum of three cells. In addition, the Nyquist Plots of impedance data are of a single cell representative of the average impedance response observed by all of the cells tested of a particular electrode and electrolyte combination. Moreover, the cell potential versus state of lithiation plots are also of a single cell representative of the cells tested. Finally, the parameters of the EIS equivalent circuit fits reported in Table 4-8 at the end of Section 4.4.5 are an average of a minimum of three cells.

\subsubsection{Lithium Titanate Oxide Half Cells}

Figure 4-32 shows the lithiation and delithiation capacity and the coulombic efficiency of the LTO half cells. The reported data is the average of three cells for each electrolyte. Before cycling, the E1 cells had an open circuit potential (OCP) on average of $2.9 \mathrm{~V}$ vs. $\mathrm{Li} / \mathrm{Li}^{+}$, while the $\mathrm{B}$ cells were $3.2 \mathrm{~V}$ vs. $\mathrm{Li} / \mathrm{Li}^{+}$. The cause of the difference between the OCP of the cells is unclear and may be due to the lower ionic conductivity of the E1 electrolyte. The E1 cells have a lower first cycle CE $(83.6 \%)$ compared to B cells $(89.4 \%)$. The low

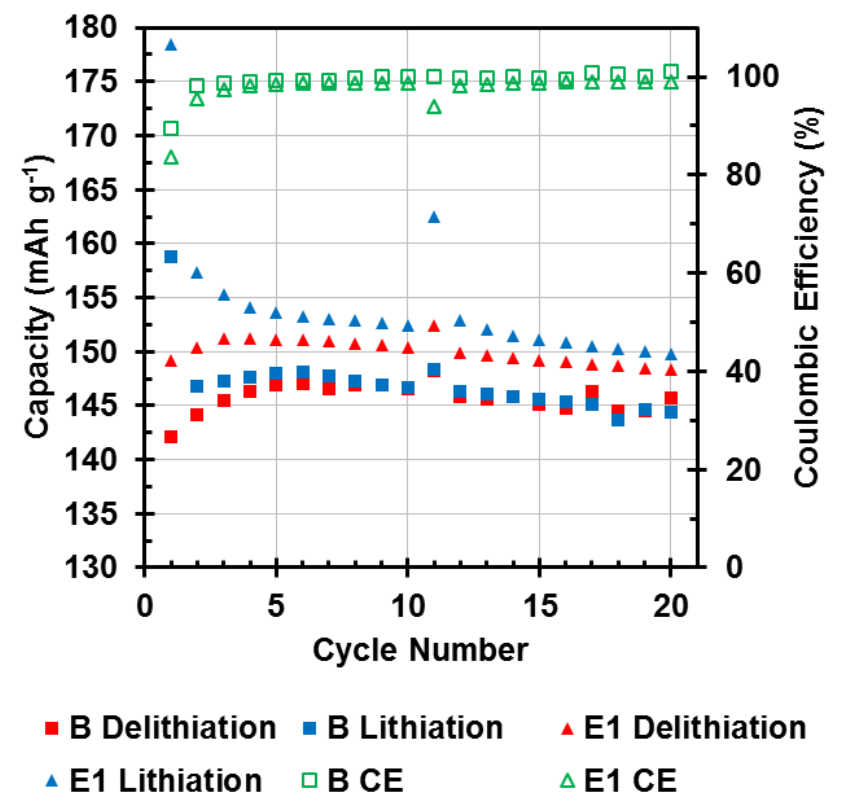

Figure 4-32: Lithiation and Delithiation Capacity and Coulombic Efficiency of LTO/Li Cells 
coulombic efficiency of the first cycle is expected for LTO, given inevitable impurities in the active material particles [120]. In addition, the E1 LTO cells require 3 cycles to achieve a CE of greater than $98 \%$, as compared to 1 cycle for B cells. This indicates that the components of E1 are not as effective at initially passivating the LTO particle surface. However, for all 20 cycles the E1 cells had a greater charge-discharge capacity than the B cells. A similar trend for improved chargedischarge performance for an HFE-containing electrolyte over a carbonate-only electrolyte was observed by Yan et al. [129]. The authors introduced a new HFE, 1,3-(1,1,2,2tetrafluoroethoxy)propane, into lithium-ion electrolytes and found that in graphite/Li cells, the HFE-containing electrolyte, EEH, (1 M LiPF 6 in 1:1:1 EC/EMC/HFE by wt) had greater chargedischarge capacity than a $1 \mathrm{M} \mathrm{LiPF}_{6}$ 3:7 EC/EMC by wt electrolyte, named EE. The authors attributed to improved performance of EEH to the improved surface wetting of the graphite due to the surfactant properties of the HFE. The HFE was measured to have a surface tension of 24.65 $\mathrm{mN} \mathrm{m}^{-1}$ which yielded a surface tension of $27.94 \mathrm{mN} \mathrm{m}^{-1}$ for the EEH electrolyte mixture, while EE mixture had a surface tension of $31.04 \mathrm{mN} \mathrm{m}^{-1}$. 3M reports the surface tension of HFE-7000 at $12.4 \mathrm{mN} \mathrm{m}^{-1}$. This suggests that $\mathrm{E} 1$ has improved surface wetting of the LTO particle surface over the baseline due to the lower surface tension of HFE-7000, possibly accounting for the greater charge-discharge capacity.

Figure 4-33a-b shows the measured impedance spectra for the LTO half cells at the lower and upper voltage limit, respectively. The resulting impedance spectra show only one characteristic time constant for the applied frequency range, which is expected for the complete lithiation and delithiation of the LTO active material. Detailed EIS studies have shown three characteristic time constants exist during the two-phase LTO lithiation-delithiation processes 
depending upon the state of lithiation [130]. The EIS spectra obtained in this work were primarily evaluated for the cell resistance, charge-transfer resistance, and double layer capacitance.

It can clearly be seen that the $\mathrm{E} 1$ cells have a higher cell resistance $\left(R_{\Omega}\right.$ in Table $\left.4-8\right)$, which is attributed to the lower measured ionic conductivity compared to B. This trend was seen for every cell combination studied with E1. Arai saw a similarly increased cell resistance for electrolytes that contained HFE-7100 as an electrolyte co-solvent and also showed that the ionic conductivity decreased with increasing amounts of the HFE in the mixture [85, 86]. Interestingly, the impedance associated with the $R_{\mathrm{ct}} \mathrm{CPE}$ circuit of the E1 cells dropped significantly from 10 to 20 cycles. The charge transfer resistance $\left(R_{\mathrm{ct}}\right)$ reduced by nearly one half while the double-layer capacitance $(Q)$ doubled, indicating an increase in the ionic conductivity of the SEI and an increase of available LTO particle active surface area. This result suggests that the initial LTO electrode-electrolyte passivation products formed during the first three cycles are not stable; however, with additional cycling a more optimal SEI forms. Yan et al. [129] in their study of the EEH electrolyte found that the SEI formed on graphite contained more organic compounds than a EEF electrolyte ( $1 \mathrm{M} \mathrm{LiPF}_{6}$

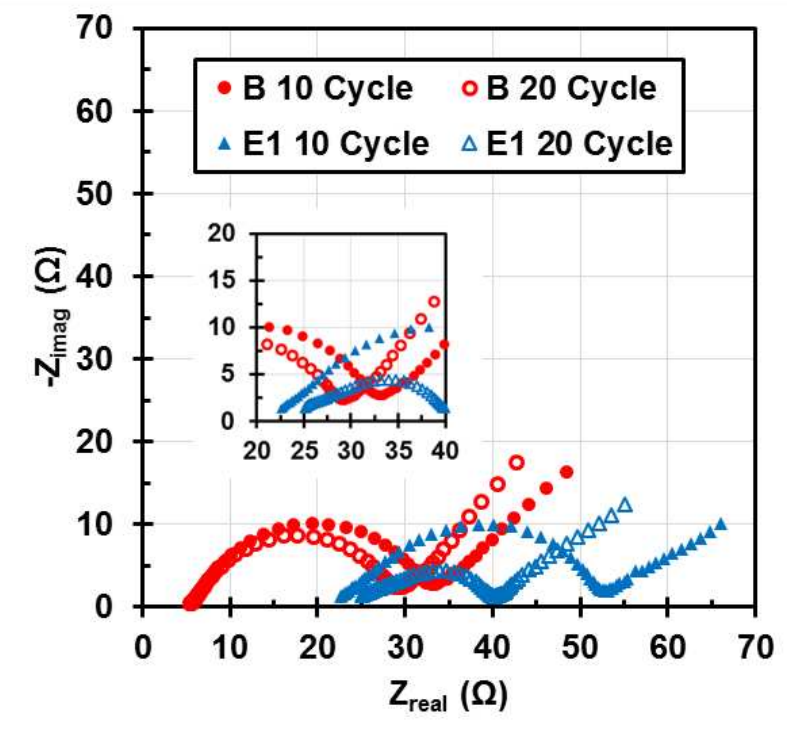

(a)

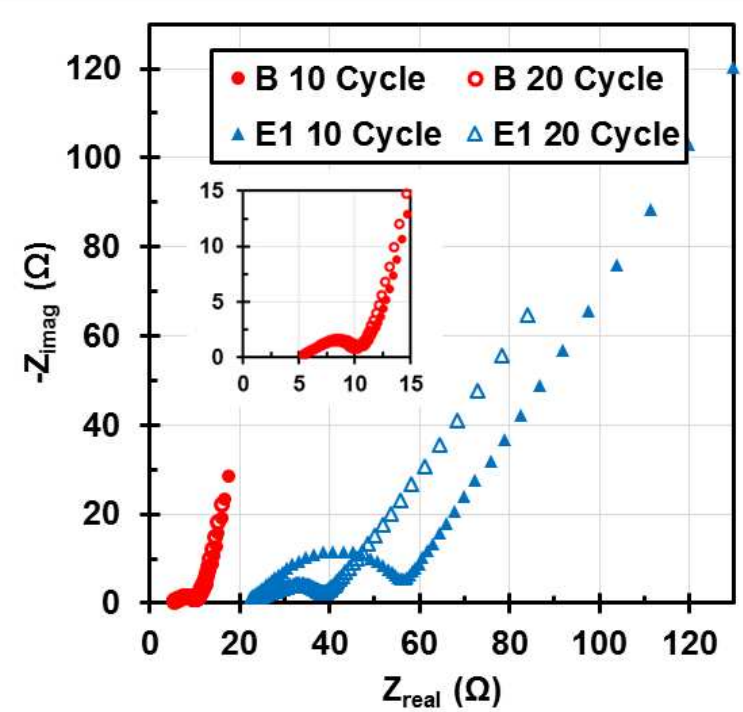

(b)

Figure 4-33: (a) Nyquist Plot at $1 \mathrm{~V}$ vs. $\mathrm{Li} / \mathrm{Li}^{+}$After 10 and 20 Cycles (b) Nyquist Plot at $2 \mathrm{~V}$ vs. $\mathrm{Li} / \mathrm{Li}^{+}$After 10 and 20 Cycles for LTO/Li Cells 
in 1:1:1 EC/EMC/FEC by wt) by measuring the higher presence of carbon and lower presence of fluorine with XPS. The lower conductivity of inorganic compounds such as LiF formed in the presence of extra fluorine lead to SEI with higher interfacial impedance. The lower SEI impedance observed by Yan with the EEH electrolyte are consistent with the results found here with E1 in LTO/Li cells.

Figure 4-34 shows the $10^{\text {th }}$ and $20^{\text {th }}$ cycle $\mathrm{LTO} / \mathrm{Li}$ cell voltage as a function of state of lithiation. The data was plotted by normalizing the capacity to the lithiation capacity measured on the $10^{\text {th }}$ and $20^{\text {th }}$ cycle for each cell. It can be seen in Figure 4-34a that the E1 cells have a slightly higher overpotential over the entire state of lithiation that is directly the result of the higher cell resistance. More interestingly, in Figure 4-34b, the E1 cell appears to deliver more than 25\% of its capacity below the characteristic two-phase voltage plateau of $\mathrm{LTO}$ of $1.55 \mathrm{~V}$ vs. $\mathrm{Li} / \mathrm{Li}^{+}$[14]. The two-phase reaction of spinel $\mathrm{Li}_{4} \mathrm{Ti}_{5} \mathrm{O}_{12}$ to $\mathrm{Li}_{7} \mathrm{Ti}_{5} \mathrm{O}_{12}$ rock salt occurs only at the $1.55 \mathrm{~V}$ plateau [131]. It appears this change to the lithiation potential indicates the E1 electrolyte causes a modification to the LTO active material. It is thought that the decomposition reactions at the

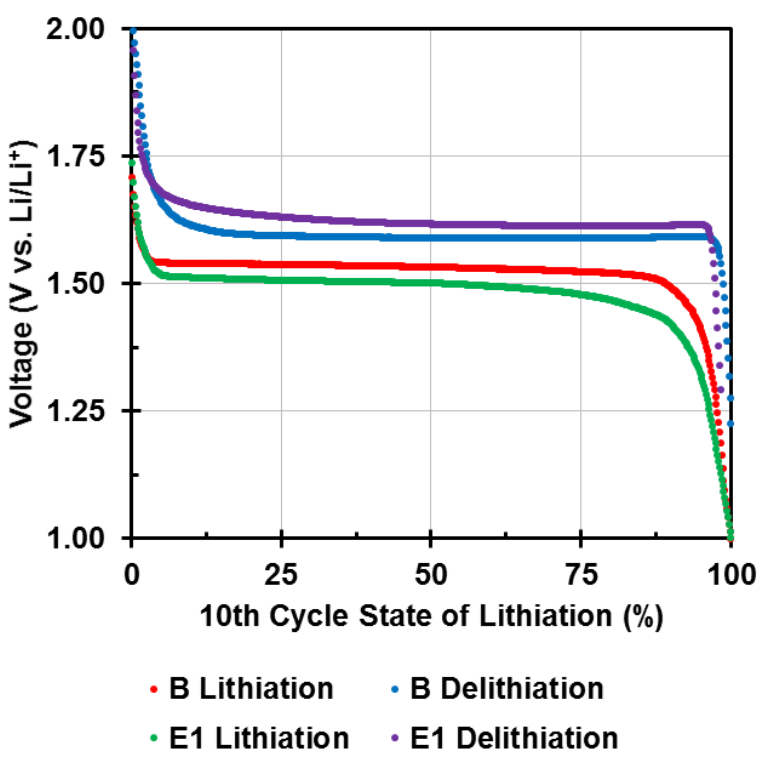

(a)

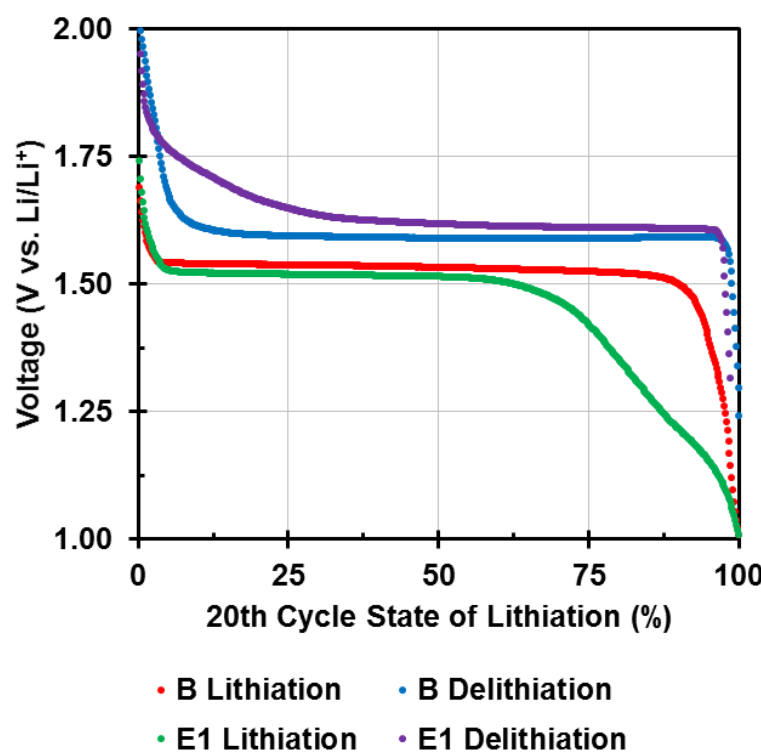

(b)

Figure 4-34: LTO/Li Cell Voltage vs. State of Lithiation (a) $10^{\text {th }}$ Cycle (b) $20^{\text {th }}$ Cycle 
electrode interface produces a $\mathrm{TiO}_{2}$ lithium intercalating structure. The single-phase lithiation of a bronze-phase $\mathrm{TiO}_{2}$ structure has a very similar sloping voltage versus capacity at the potentials seen in the $\mathrm{E} 1$ half cell. Yan et al. credits the capacity in in $\mathrm{TiO}_{2}$ at these potentials to partial lithium storage at the surface of the active material particles [132]. The formation of the $\mathrm{TiO}_{2}$-like intercalating compound occurs between cycles $11-20$ as evidenced by the change in the $R_{\mathrm{ct}}$ and CPE from 10 to 20 cycles. Based on the cycling capacity of the E1 LTO cells, the introduction of this alternate lithium-intercalating phase is not detrimental to cell reversibility. Further cycling is required to evaluate the impact of E1 on LTO, and if the observed lithiation potential change is detrimental to long-term reversibility.

\subsubsection{Copper Antimonide Half Cells}

Figure 4-35 shows the cycling and coulombic efficiency performance of $\mathrm{Cu}_{2} \mathrm{Sb} / \mathrm{Li}$ cells. Before cycling, the E1 cells had an average OCP of $2.8 \mathrm{~V}$ vs. $\mathrm{Li} / \mathrm{Li}^{+}$while the B cells had an average of $3.3 \mathrm{~V}$ vs. $\mathrm{Li} / \mathrm{Li}^{+}$. The lower OCP for the $\mathrm{E} 1$ cells is again unclear and could possibly be

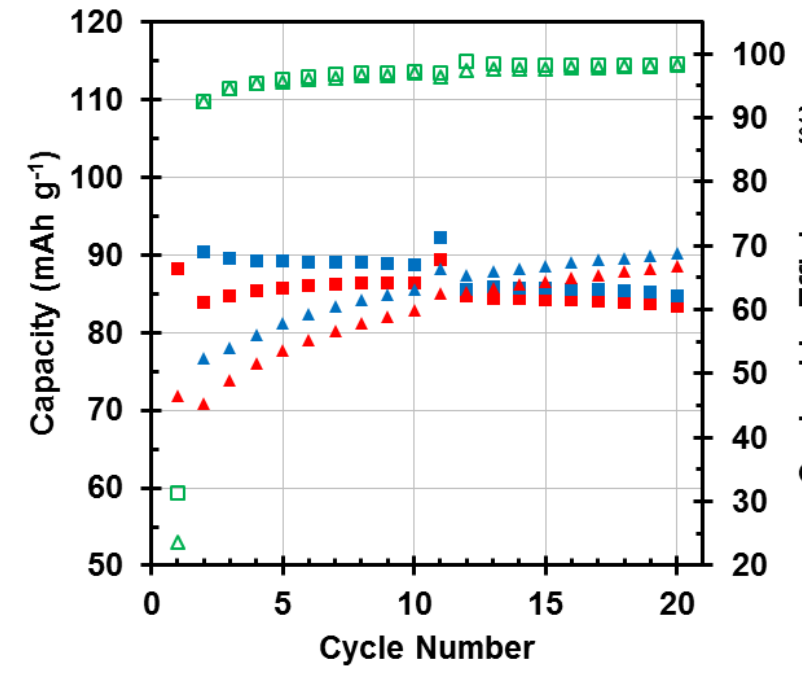

- B Delithiation - B Lithiation

$\triangle \mathrm{E} 1$ Lithiation $\square \mathrm{BCE}$ $\triangle$ E1 Delithiation $\triangle \mathrm{E} 1 \mathrm{CE}$ attributed to the lower ionic conductivity of the electrolyte. For improved reversibility, the $\mathrm{Cu}_{2} \mathrm{Sb}$ electrode was only lithiated to 0.5 $\mathrm{V}$ vs. $\mathrm{Li} / \mathrm{Li}^{+}$to minimize the formation of $\mathrm{Li}_{3} \mathrm{Sb} . \mathrm{Li}_{3} \mathrm{Sb}$ formation and subsequent copper extrusion is the only capacityproducing reaction occurring at voltages below $0.5 \mathrm{~V}$ vs. $\mathrm{Li} / \mathrm{Li}^{+}$for the intermetallic electrode $[17,133]$. It has been shown that

Figure 4-35: Lithiation and Delithiation complete copper extrusion from the $\mathrm{Cu}_{2} \mathrm{Sb}$ Capacity and Coulombic Efficiency of $\mathrm{Cu}_{2} \mathrm{Sb} / \mathrm{Li}$ Cells 
structure causes severe capacity degradation as the $\mathrm{Li}_{3} \mathrm{Sb}$ can become electrically isolated from the electrode [128]. The first lithiation cycle of the cells was performed at a fifth of the cycling current density. The E1 cells had a first cycle lithiation capacity of $312 \mathrm{mAh} \mathrm{g}^{-1}$, while the B cells had a capacity of $280 \mathrm{mAh} \mathrm{g}^{-1}$. High first lithiation capacity loss has been observed for $\mathrm{Cu}_{2} \mathrm{Sb}$ anodes manufactured from powders and is attributed to electrolyte reaction to oxides formed on the surface of the active material particles during manufacture $[17,128,133,134]$. However, the direct electrodeposition of $\mathrm{Cu}_{2} \mathrm{Sb}$ onto the copper current collector does significantly reduce the amount of surface oxides formed, so the irreversible capacity loss can be primarily attributed to electrolyte decomposition for SEI formation. The first lithiation capacity difference can be attributed to the reduced passivation capability of E1 compared to B.

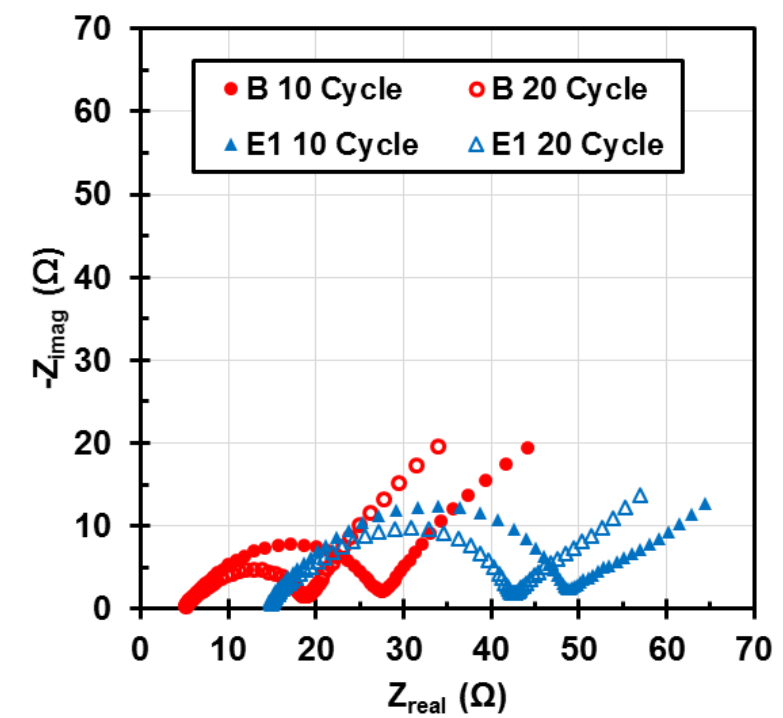

(a)

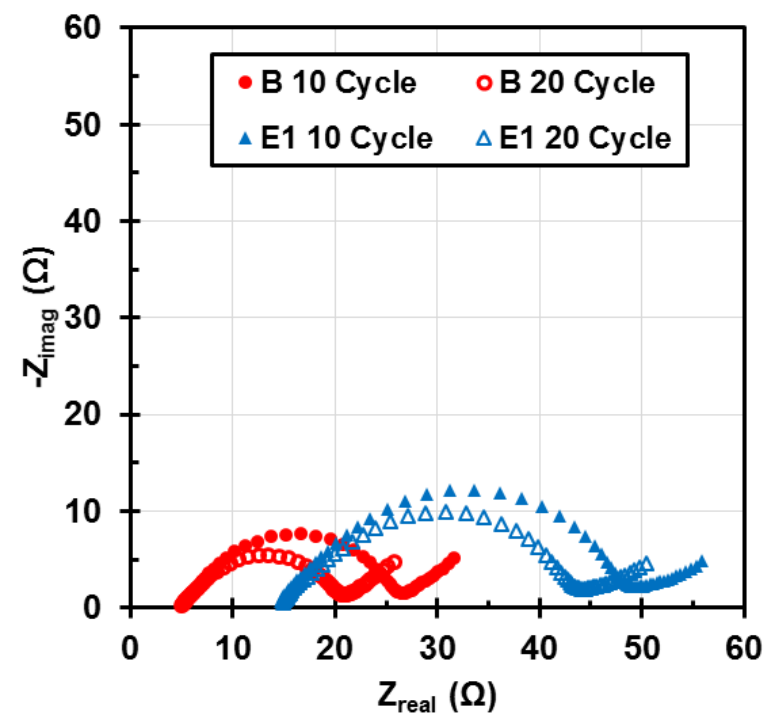

(b)

Figure 4-36: (a) Nyquist Plot at $0.5 \mathrm{~V}$ vs. $\mathrm{Li} / \mathrm{Li}^{+}$After 10 and $20 \mathrm{Cycles}$ (b) Nyquist Plot at $0.95 \mathrm{~V}$ vs. $\mathrm{Li} / \mathrm{Li}^{+}$After 10 and 20 Cycles for $\mathrm{Cu} 2 \mathrm{Sb} / \mathrm{Li}$ Cells

Both $\mathrm{E} 1$ and $\mathrm{B} \mathrm{Cu}_{2} \mathrm{Sb} / \mathrm{Li}$ cells experienced increasing coulombic efficiency as cycle number increased. Moreover, the E1 cells saw a significant increase in the reversible capacity as cycle number increased: an approximately $19 \mathrm{mAh} \mathrm{g}^{-1}$ increase from cycle 2 to 20 . This can be attributed to the decreasing impedance of the cell as seen in Figure 4-36a-b, which enables lithium 


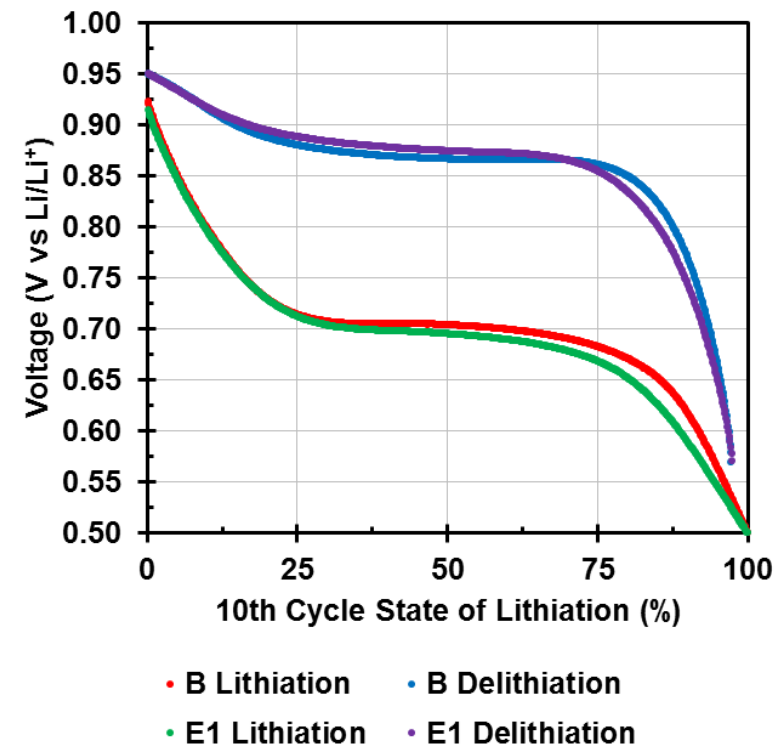

(a)

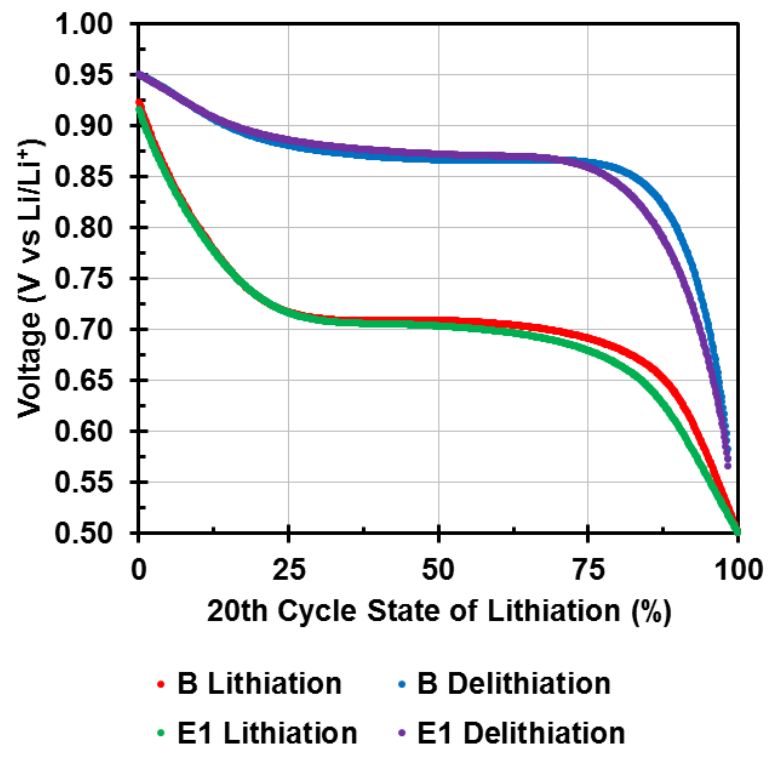

(b)

Figure 4-37: Cu2Sb/Li Cell Voltage vs. State of Lithiation (a) $10^{\text {th }}$ Cycle (b) $20^{\text {th }} \mathrm{Cycle}^{\mathrm{S}}$

trapped during the first lithiation of the electrode to be accessed. Bryngelsson et al. saw capacity increase in their $\mathrm{Cu}_{2} \mathrm{Sb}$ electrodes produced by deposition in $\mathrm{pH} 1.3$ solutions as well, although no proposed mechanism was provided [135]. Table 4-8 shows an approximate $20 \%$ decrease in $R_{\mathrm{ct}}$ for E1 cells indicating the ionic conductivity of the SEI improves. A similar decrease in $R_{\mathrm{ct}}$ is observed for B cells; however, this does not result in improved cycling capacity as a slight capacity fade is observed throughout the 20 cycles. This result is similar to that found by Song et al. [128] who studied $\mathrm{Cu}_{2} \mathrm{Sb} / \mathrm{Li}$ cell cycling with a similarly-composed $\mathrm{LiPF}_{6}$ EC/DEC electrolyte. Figure 4-37a-b shows the $10^{\text {th }}$ and $20^{\text {th }}$ cycle voltage as a function of state of lithiation for the $\mathrm{Cu}_{2} \mathrm{Sb} / \mathrm{Li}$ cells. Both the E1 and B cells have very similar voltage profiles and overpotentials at both the $10^{\text {th }}$ and $20^{\text {th }}$ cycle. This indicates identical utilization of the two-phase region of $\mathrm{Cu}_{2} \mathrm{Sb}$. 


\subsubsection{Lithium Iron Phosphate Half Cells}

Figure 4-38 shows the cycling and coulombic efficiency performance of LFP/Li cells. LFP was selected as a cathode active material because of its relatively low redox potential (3.5 V vs. $\mathrm{Li} / \mathrm{Li}^{+}$) versus other lithium intercalating cathode chemistries. The choice of LFP precludes the need to form a substantial SEI because the oxidative limit of the electrolyte is nearly $1 \mathrm{~V}$ above the upper voltage limit of the $\mathrm{LFP} / \mathrm{Li}$ cell $\left(4 \mathrm{~V}\right.$ vs. $\left.\mathrm{Li} / \mathrm{Li}^{+}\right)$. The lower voltage limit $(2.5 \mathrm{~V}$ vs. $\mathrm{Li} / \mathrm{Li}^{+}$) of the LFP half cell is also much greater than the reduction limit of the electrolytes allowing both the E1 (94\%) and B (96\%) cells have a much higher first cycle CE compared to LTO and $\mathrm{Cu}_{2} \mathrm{Sb}$ half cells. However, after approximately six cycles, the reversible capacity begins to fade for the B cells, and more dramatically so for the E1 cells. The capacity fade seen in the B cells can possibly be attributed to two degradation mechanisms: trace water contamination in the cell and LFP active material particle cracking. The $\mathrm{PF}_{6}{ }^{-}$anions in the $\mathrm{B}$ electrolyte readily react with any trace $\mathrm{H}_{2} \mathrm{O}$ to form $\mathrm{HF}$ which is capable of dissolving iron from the olivine structure of LFP, reducing the capacity of the cell [136].

Wang et al. showed that significant capacity fade occurred in LFP/Li cells with a $1.0 \mathrm{M}$ $\mathrm{LiPF}_{6}$ EC/DMC electrolyte that was attributed to LFP particle fractures from the volume expansion due to lithium intercalation and de-intercalation processes [137]. The authors utilized the entire capacity of the LFP active material using voltage limits of $2-4.5 \mathrm{~V}$ vs. $\mathrm{Li} / \mathrm{Li}^{+}$. Similar

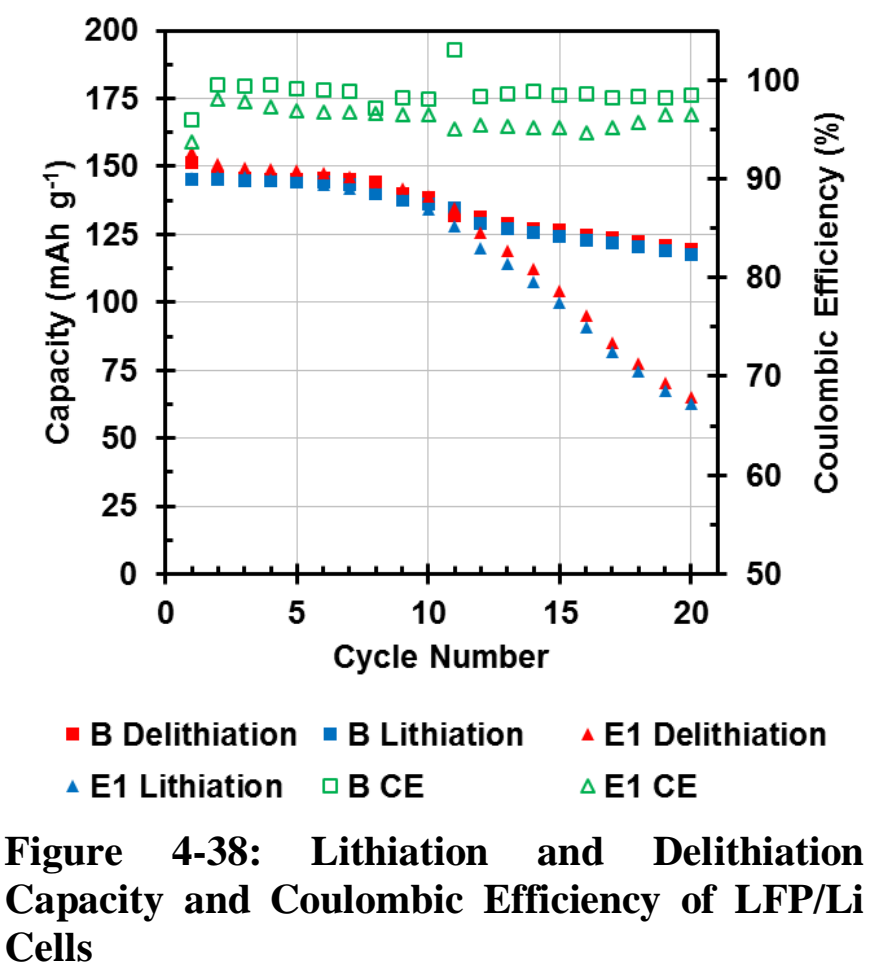


total capacity utilization of the LFP particle was used in this study with the cycling voltage limits of 2.5-4 V vs. $\mathrm{Li} / \mathrm{Li}^{+}$. Either of these mechanisms are plausible; however, the capacity degradation rate observed reflects very similarly to the LFP particle fracture observed by Wang.

The capacity fade seen in the E1 cells is attributed to the interaction of the aluminum current collector and the LiTFSI salt. 1.0 M concentrations of LiTFSI has been shown to cause aluminum current collector corrosion at potentials above $3.8 \mathrm{~V}$ vs. $\mathrm{Li} / \mathrm{Li}^{+}$in organic carbonate solvents [106, 107, 138]. It was unclear if the upper voltage limit of $4 \mathrm{~V}$ vs. $\mathrm{Li} / \mathrm{Li}^{+}$would cause aluminum corrosion in the E1 cells or if the fluorinated HFE-7000 co-solvent would be capable of providing aluminum passivation by reacting to form LiF. Based on the results, it is clear that HFE7000 does not assist in aluminum current collector passivation as the cell impedance continues to grow over the 20 cycles of the cell.

Figure 4-39 shows the dramatic growth in impedance in an E1 cell from 10 to 20 cycles. Most notably, a 64\% increase in $R_{\mathrm{ct}}$ is observed at $4 \mathrm{~V}$ vs. $\mathrm{Li} / \mathrm{Li}^{+}$for $\mathrm{E} 1$ cells from cycles 10 to 20. This indicates that irreversible aluminum corrosion is occurring at this potential and the E1 mixture

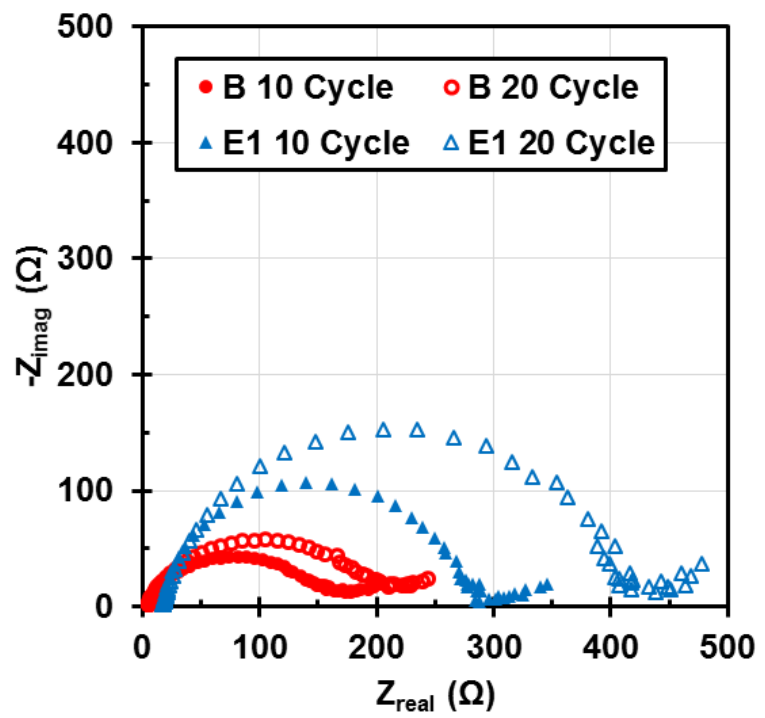

(a)

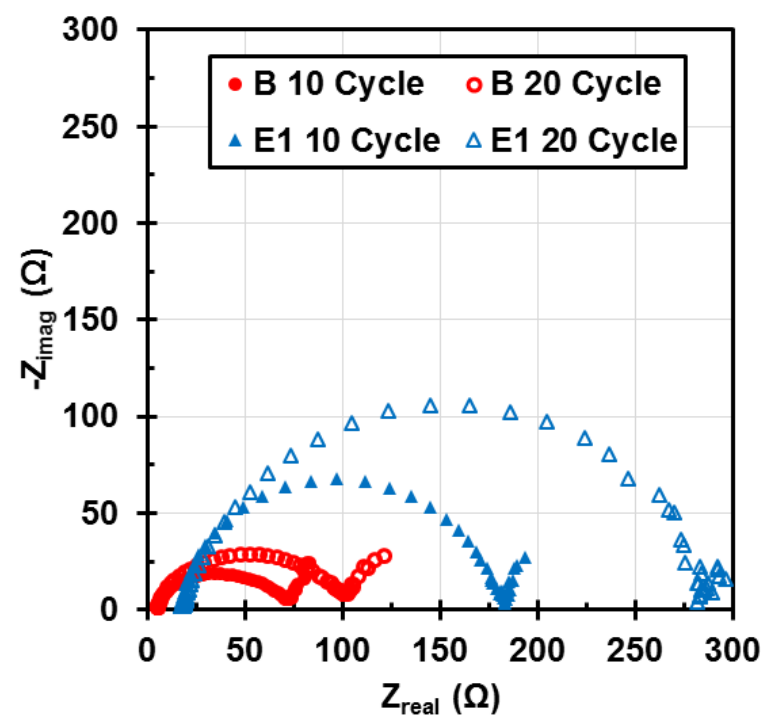

(b)

Figure 4-39: (a) Nyquist Plot at $2.5 \mathrm{~V}$ vs. Li/Li ${ }^{+}$After 10 and 20 Cycles (b) Nyquist Plot at 4 V vs. $\mathrm{Li} / \mathrm{Li}^{+}$After 10 and 20 Cycles for $\mathrm{LFP} / \mathrm{Li}$ Cells 
is not capable of passivating the surface of the aluminum, as the electrolyte continues to decompose.

This effect is also very evident in Figure 4-40 which shows the voltage versus state of lithiation for the $10^{\text {th }}$ and $20^{\text {th }}$ cycle for both electrolytes. The large voltage difference between the lithiation and delithiation curves for E1 indicates large cell resistances resulting from the aluminum corrosion and electrolyte decomposition, which clearly worsens from cycle 10 to 20. Several solutions have been identified for aluminum passivation in 1.0 M LiTFSI electrolytes. The addition of another lithium salt, either $1 \%$ by electrolyte weight $(0.1 \mathrm{M}) \mathrm{LiPF}_{6}[138]$ or $0.26 \mathrm{M}$ of $\mathrm{LiBOB}$ [139] has proven to be sufficient for aluminum passivation, and further investigation is warranted for E1-containing LFP/Li cells.

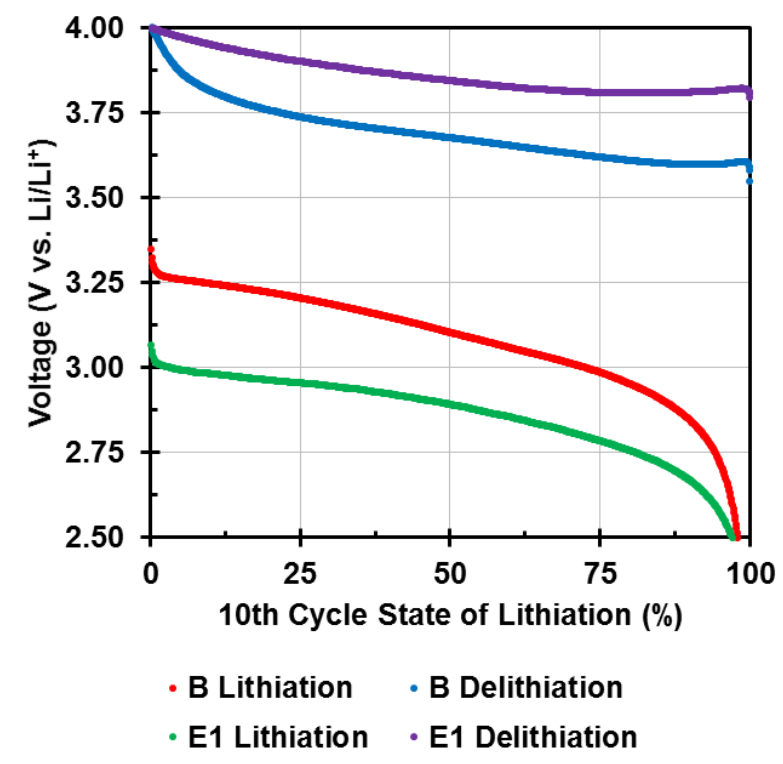

(a)

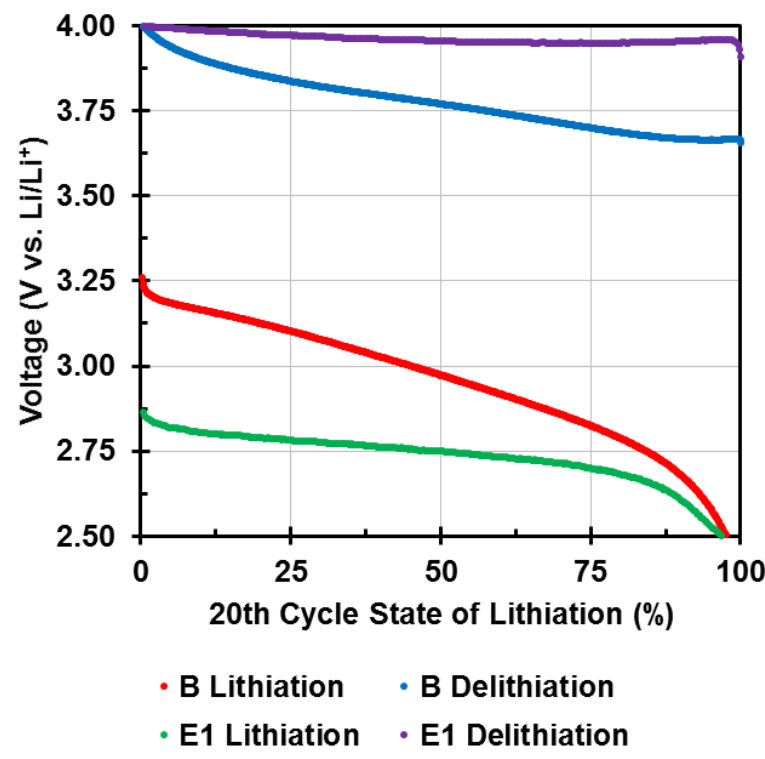

(b)

Figure 4-40: LFP/Li Cell Voltage vs. State of Lithiation (a) $10^{\text {th }}$ Cycle (b) $20^{\text {th }}$ Cycle 
Table 4-8: Average EIS Fit Parameters for Half Cells, All Potentials are vs. $\mathrm{Li} / \mathrm{Li}^{+}$

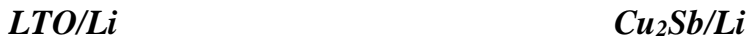

\begin{tabular}{|c|c|c|c|c|c|c|c|c|c|c|c|c|c|}
\hline \multirow[b]{3}{*}{$\begin{array}{l}\text { Model } \\
\text { Parameter } \\
\end{array}$} & \multirow[b]{3}{*}{ Electrolyte } & \multirow{2}{*}{\multicolumn{4}{|c|}{20 Cvcle }} & & \multirow{2}{*}{\multicolumn{2}{|c|}{10 Cycle }} & \multirow{2}{*}{\multicolumn{2}{|c|}{20 Cycle }} \\
\hline & & & & & & 10 & ycle & & cle & & & & \\
\hline & & $1 \mathrm{~V}$ & $2 \mathrm{~V}$ & $1 \mathrm{~V}$ & $2 \mathrm{~V}$ & $0.5 \mathrm{~V}$ & $0.95 \mathrm{~V}$ & $0.5 \mathrm{~V}$ & $0.95 \mathrm{~V}$ & $2.5 \mathrm{~V}$ & $4 \mathrm{~V}$ & $2.5 \mathrm{~V}$ & $4 \mathrm{~V}$ \\
\hline \multirow{2}{*}{$\begin{array}{l}R_{\Omega} \\
{[\Omega]}\end{array}$} & $\mathrm{B}$ & 5.78 & 5.77 & 6.15 & 6.17 & 4.74 & 4.60 & 4.96 & 4.77 & 4.43 & 4.51 & 4.79 & 4.86 \\
\hline & E1 & 18.6 & 18.7 & 20.5 & 20.0 & 14.4 & 14.5 & 14.9 & 14.8 & 17.6 & 17.7 & 18.7 & 18.9 \\
\hline \multirow{2}{*}{$\begin{array}{c}Q \times \mathbf{1 0}^{4} \\
{\left[\mathrm{~S} \mathrm{~s}^{\alpha}\right]}\end{array}$} & $\mathrm{B}$ & 1.33 & 2.85 & 1.26 & 3.28 & 1.42 & 2.41 & 1.64 & 3.09 & 0.403 & 0.382 & 0.420 & 0.369 \\
\hline & E1 & 0.662 & 0.407 & 1.57 & 1.17 & 0.519 & 0.878 & 0.660 & 1.30 & 0.172 & 0.167 & 0.213 & 0.206 \\
\hline \multirow{2}{*}{$\begin{array}{c}\alpha \\
{[-]}\end{array}$} & $\mathrm{B}$ & 0.790 & 0.825 & 0.796 & 0.795 & 0.706 & 0.683 & 0.699 & 0.660 & 0.765 & 0.794 & 0.763 & 0.793 \\
\hline & E1 & 0.740 & 0.785 & 0.663 & 0.668 & 0.752 & 0.725 & 0.746 & 0.701 & 0.821 & 0.833 & 0.802 & 0.807 \\
\hline \multirow{2}{*}{$\begin{array}{c}W_{\mathrm{d}} \\
{\left[\mathbf{S ~ s}^{1 / 2}\right]}\end{array}$} & B & 5.13 & 7.63 & 14.6 & 8.16 & 4.26 & 17.3 & 4.53 & 17.0 & 1.59 & 2.49 & 1.51 & 2.14 \\
\hline & E1 & 7.73 & 1.21 & 6.66 & 1.87 & 5.96 & 13.4 & 6.56 & 14.5 & 1.97 & 2.91 & 3.49 & 11.1 \\
\hline \multirow{2}{*}{$\begin{array}{c}R_{\mathrm{ct}} \\
{[\boldsymbol{\Omega}]}\end{array}$} & $\mathrm{B}$ & 25.9 & 3.16 & 24.0 & 3.83 & 25.47 & 20.0 & 18.7 & 18.6 & 142 & 53.6 & 177 & 75.6 \\
\hline & E1 & 34.2 & 34.5 & 17.3 & 16.0 & 38.0 & 35.1 & 28.6 & 29.6 & 287 & 185 & 377 & 305 \\
\hline \multirow{2}{*}{$\begin{array}{c}f_{\mathrm{c}} \\
{[\mathrm{Hz}]}\end{array}$} & B & 210 & 784 & 234 & 710 & 456 & 392 & 631 & 397 & 136 & 387 & 98 & 264 \\
\hline & E1 & 601 & 689 & 1170 & 1946 & 627 & 462 & 713 & 442 & 103 & 164 & 65 & 85 \\
\hline
\end{tabular}




\subsection{Full Cell Electrochemical Testing}

Upon completion of half cell electrochemical testing, full cells were constructed to study the impact of the candidate MFE with only lithium-ion insertion active materials (LFP, LTO, and $\mathrm{Cu}_{2} \mathrm{Sb}$ ). Full cell electrochemical testing provides insight into the coupled positive electrodeelectrolyte-negative electrode performance that is consistent with a commercial LIB, where no lithium metal is present. The full cell construction process, experimental techniques, and resulting data are described in the following sections.

\subsubsection{Definition and Method of Measurement}

Full cells were constructed

with the following combinations:

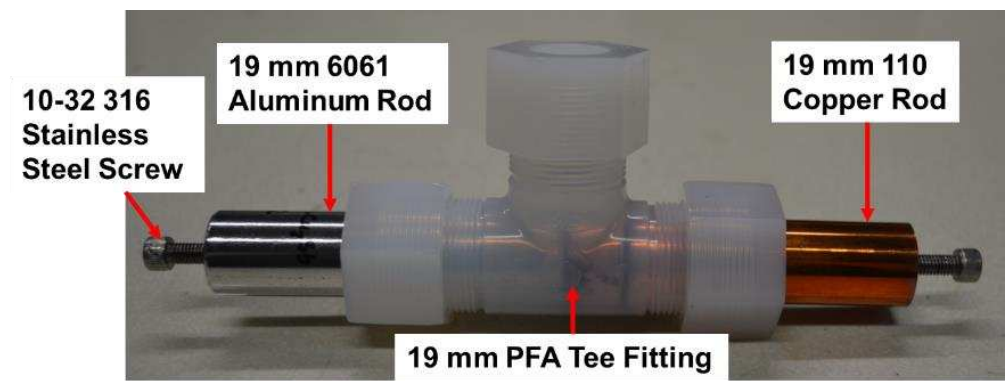

$\mathrm{LFP} / \mathrm{Cu}_{2} \mathrm{Sb}$ and $\mathrm{LFP} / \mathrm{LTO}$. The

architecture of the full cell is nearly

identical to that of the half cell Figure 4-41: Assembled Full Cell

depicted in Figure 4-17. The same 19 mm PFA T-fitting and electrical connection scheme is used.

For a full cell, the lithium metal electrode is replaced with a negative electrode, either LTO or $\mathrm{Cu}_{2} \mathrm{Sb}$. In addition, the $19 \mathrm{~mm} 316$ stainless steel rods used to electrically interface with the lithium metal in half cells are replaced with $19 \mathrm{~mm} 101$ copper rods. An assembled full cell is seen in Figure 4-41; note the aluminum rods interface with the positive electrode, LFP, and the copper rods interface with the negative electrode ( $\mathrm{LTO}$ or $\mathrm{Cu}_{2} \mathrm{Sb}$ ). The LFP, LTO, and $\mathrm{Cu}_{2} \mathrm{Sb}$ electrodes used in full cells are produced by the same manufacturing processes described in Sections 4.4.4.1 and 4.4.4.2. 


\subsubsection{Test Parameters and Equipment Used}

In both the $\mathrm{LFP} / \mathrm{Cu}_{2} \mathrm{Sb}$ and LFP/LTO architectures, the anode active material was the limiting capacity. The cycling voltage limits for the $\mathrm{LFP} / \mathrm{Cu}_{2} \mathrm{Sb}$ full cells were determined from the half cell cycling voltage limits. The upper voltage limit of the full cell $(3.5 \mathrm{~V})$ was determined by subtracting the delithiated LFP cathode potential $\left(4 \mathrm{Vvs}\right.$. $\left.\mathrm{Li} / \mathrm{Li}^{+}\right)$from the lithiated potential of the $\mathrm{Cu}_{2} \mathrm{Sb}$ anode $\left(0.5 \mathrm{~V}\right.$ vs. $\left.\mathrm{Li} / \mathrm{Li}^{+}\right)$. The lower voltage limit assumed the $\mathrm{LFP}$ cathode potential would remain near $3.5 \mathrm{~V}$ vs. $\mathrm{Li} / \mathrm{Li}^{+}$due to the limiting capacity of the $\mathrm{Cu}_{2} \mathrm{Sb}$ anode, and its potential would quickly rise when fully delithiated. The delithiated potential for the $\mathrm{Cu}_{2} \mathrm{Sb}$ anode was determined to be $1.75 \mathrm{~V}$ vs. $\mathrm{Li} / \mathrm{Li}^{+}$, yielding $1.75 \mathrm{~V}$ as the lower voltage limit of the $\mathrm{LFP} / \mathrm{Cu}_{2} \mathrm{Sb}$ cell. The cycling voltage limits for the LFP/LTO full cells were determined by predicting the average potential of the cell to be approximately $2 \mathrm{~V}$ since LFP has a two-phase voltage plateau of $3.5 \mathrm{~V}$ vs. $\mathrm{Li} / \mathrm{Li}^{+}$and $\mathrm{LTO}$ has a two-phase voltage plateau of $1.5 \mathrm{~V}$ vs. $\mathrm{Li} / \mathrm{Li}^{+}$. Because minimal capacity is delivered outside of the two-phase region for both active materials, the LFP/LTO voltage limits were extended by $0.5 \mathrm{~V}$ on either side of $2 \mathrm{~V}$.

The same style of generic test plan for galvanostatic cycling used for half cells was used for full cells (Table 4-6). The cells were cycled 10 times, removed from the Arbin battery tester and connected to the Gamry Reference 3000 potentiostat for EIS measurements at the UVL and LVL of the cells. Again, the same style of test plan was created in the Gamry Framework Sequence Editor as was used for the half cell tests (Table 4-7).

The current applied for each full cell combination was different: $291 \mu \mathrm{A} \mathrm{cm}^{-2}$ for LFP/LTO cells and $175 \mu \mathrm{A} \mathrm{cm}{ }^{-2}$ for $\mathrm{LFP} / \mathrm{Cu}_{2} \mathrm{Sb}$ cells. In addition, the $\mathrm{LFP} / \mathrm{Cu}_{2} \mathrm{Sb}$ cells were cycled at an approximate $0.1 \mathrm{C}$ rate for the first charge, $52.63 \mu \mathrm{A} \mathrm{cm}{ }^{-2}$. The determination of these cycling currents utilized a similar process to that described in Section 4.4.4.3. The initial cycling currents 
determined by Equation (4.44) were refined after galvanostatic cycling trials of full cells for each combination. Table 4-9 describes the voltage limit adjusted capacity for the full cell combination studies. The $\mathrm{LFP} / \mathrm{Cu}_{2} \mathrm{Sb}$ cell, which is capacity limited by the $\mathrm{Cu}_{2} \mathrm{Sb}$ anode, has a much greater voltage limit adjusted capacity than in a half cell architecture (Table 4-5). This indicates that additional capacity producing reactions are utilized outside of the $0.5-0.95 \mathrm{~V}$ vs. $\mathrm{Li}^{-} \mathrm{Li}^{+}$potential operating window of the $\mathrm{Cu}_{2} \mathrm{Sb} / \mathrm{Li}$ cell.

Table 4-9: Full Cell Voltage Limits and Capacity

\begin{tabular}{lccc}
$\begin{array}{l}\text { Full Cell } \\
\text { Type }\end{array}$ & $\begin{array}{c}\text { Upper Voltage } \\
\text { Limit }(\mathbf{V})\end{array}$ & $\begin{array}{c}\text { Lower Voltage } \\
\text { Limit }(\mathbf{V})\end{array}$ & $\begin{array}{c}\text { Cycling Capacity } \\
\left(\mathbf{m A h ~ g}^{-1}\right)\end{array}$ \\
\hline LFP/Cu $\mathrm{Cu}_{2} \mathrm{Sb}$ & 3.5 & 1.75 & 150 \\
\hline LFP/LTO & 2.5 & 1.5 & 140
\end{tabular}

\subsubsection{Results and Discussion}

Similar to the half cell tests, the reported data for the charge and discharge capacity and coulombic efficiency plots is an average of a minimum of three cells. In addition, the Nyquist Plots of impedance data are of a single cell representative of the average impedance response observed by all of the cells tested of a particular electrode and electrolyte combination. Moreover, the cell potential versus state of charge plots are for a single cell representative of the cells tested. Finally, the parameters of the EIS equivalent circuit fits reported in the Table 4-10 at the end of Section 4.5.3 are an average of a minimum of three cells.

\subsubsection{Lithium Iron Phosphate - Copper Antimonide Cells}

The cycling and coulombic efficiency performance of $\mathrm{LFP} / \mathrm{Cu}_{2} \mathrm{Sb}$ full cells is shown in Figure 4-42. The first charge coulombic efficiency for the E1 cells (83\%) was significantly higher than for the B cells (58\%). Throughout the 20 cycles, the E1 cells have a slightly greater coulombic efficiency suggesting that the E1 electrolyte offers improved cell reversibility. Both electrolytes showed some capacity fade over the 20 cycles. The reduction in capacity could be due to the 
sensitivity of the $\mathrm{Cu}_{2} \mathrm{Sb}$ anode on the cell voltage limits. If the potential of the $\mathrm{Cu}_{2} \mathrm{Sb}$ electrode becomes lower than $0.5 \mathrm{~V}$ vs. $\mathrm{Li} / \mathrm{Li}^{+}$in the full cell architecture, copper will be extruded from the active material structure. As previously stated, this reduces the reversible capacity of the active material independent of the electrolyte used and could be the source of capacity fade. It is likely that the potential of the $\mathrm{Cu}_{2} \mathrm{Sb}$ anode

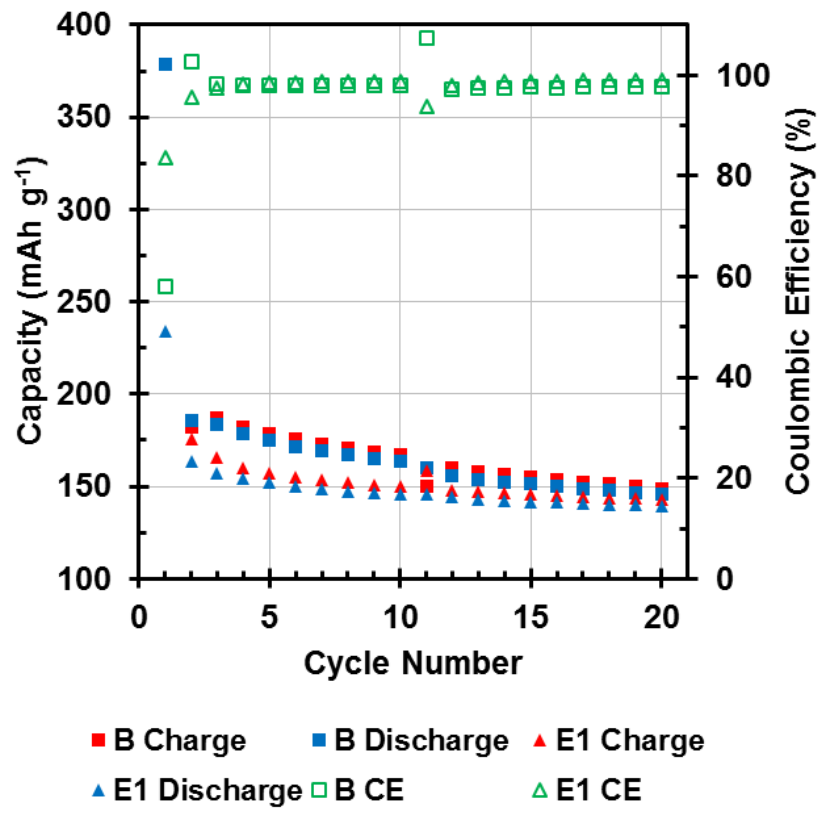

Figure 4-42: Charge and Discharge Capacity and Coulombic Efficiency of LFP/Cu2Sb Cells was lower than ideal threshold of $0.5 \mathrm{~V}$ vs. $\mathrm{Li} / \mathrm{Li}^{+}$since it was the limiting capacity electrode of the full cell. This is exemplified by the significantly greater capacity (approximately $150 \mathrm{mAh}$ $\mathrm{g}^{-1}$ ) produced by the $\mathrm{LFP} / \mathrm{Cu}_{2} \mathrm{Sb}$ cells in comparison to the $\mathrm{Cu}_{2} \mathrm{Sb} / \mathrm{Li}$ cells (approximately $90 \mathrm{mAh}$ $\mathrm{g}^{-1}$ ) which were carefully controlled between the potential limits of $0.5-0.95 \mathrm{~V} \mathrm{vs}$. $\mathrm{Li} / \mathrm{Li}^{+}$. Song et al. showed the sensitivity of the $\mathrm{Cu}_{2} \mathrm{Sb}$ anode to full cell voltage limits. The reversible capacity dramatically improved in a $\mathrm{LiNi}_{0.8} \mathrm{Co}_{0.15} \mathrm{Al}_{0.05} \mathrm{O}_{2} / \mathrm{Cu}_{2} \mathrm{Sb}$ cell with revised cell voltage limits to limit the $\mathrm{Cu}_{2} \mathrm{Sb}$ anode's potential to $0.65-1.4 \mathrm{~V}$ vs. $\mathrm{Li} / \mathrm{Li}^{+}$as opposed to $0.1-1.2 \mathrm{~V}$ vs. $\mathrm{Li} / \mathrm{Li}^{+}$[128].

Figure 4-43 shows the impedance spectra of the $\mathrm{LFP} / \mathrm{Cu}_{2} \mathrm{Sb}$ cell at the lower and upper voltage limits. The resulting equivalent circuit model fit parameters are listed in Table 4-10. Interestingly, the charge transfer resistance of the E1 cells is nearly half of the B cells at 20 cycles. Moreover, the charge transfer resistance at the upper voltage limit of the $\mathrm{LFP} / \mathrm{Cu}_{2} \mathrm{Sb}$ cell decreased as cycle number increased. This result indicates that more favorable charge transfer kinetics through the SEI are present in the E1 cells. More importantly, this result also shows that aluminum 


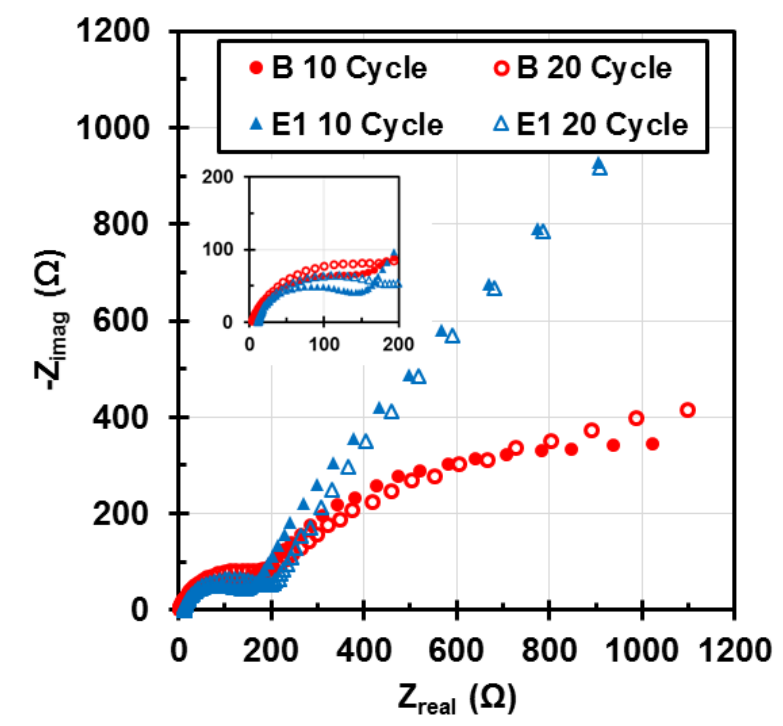

(a)

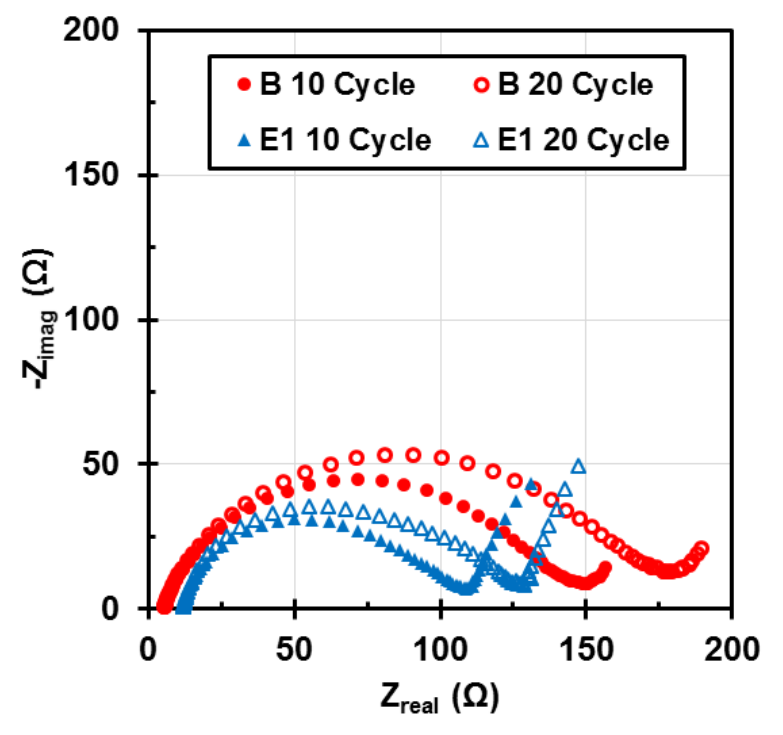

(b)

Figure 4-43: (a) Nyquist Plot at 1.75 V After 10 and 20 Cycles (b) Nyquist Plot at $3.5 \mathrm{~V}$ After 10 and 20 Cycles for $\mathrm{LFP} / \mathrm{Cu}_{2} \mathrm{Sb}$ Cells

current collector corrosion on the LFP cathode is not present in the E1-containing full cells. The E1-containing LFP/Li half cells had a growing charge transfer resistance and severe capacity fade over the 20 cycles, but this is not the case for the full cells. This is attributed to the positive electrode of the full cells never reaching a potential greater than $3.8 \mathrm{~V}$ vs. $\mathrm{Li} / \mathrm{Li}^{+}$to activate the aluminum corrosion reaction.

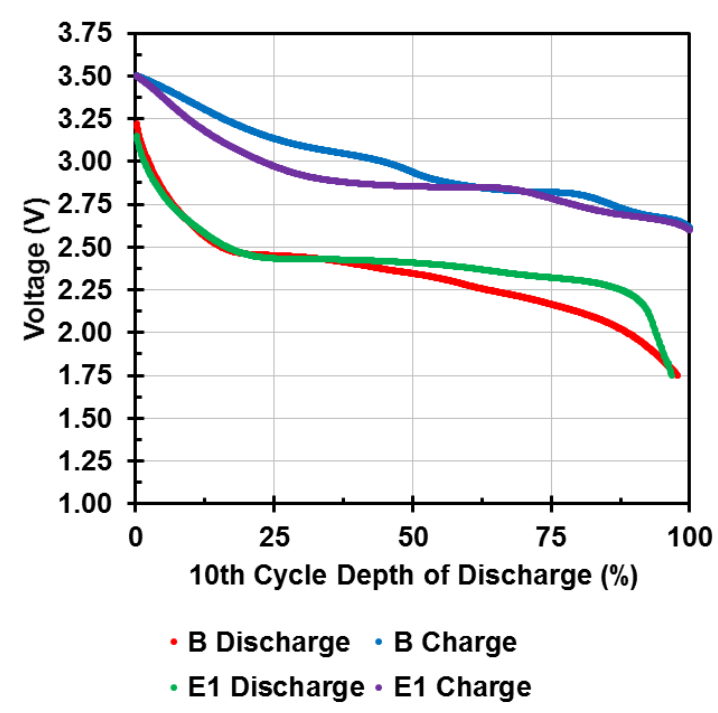

(a)

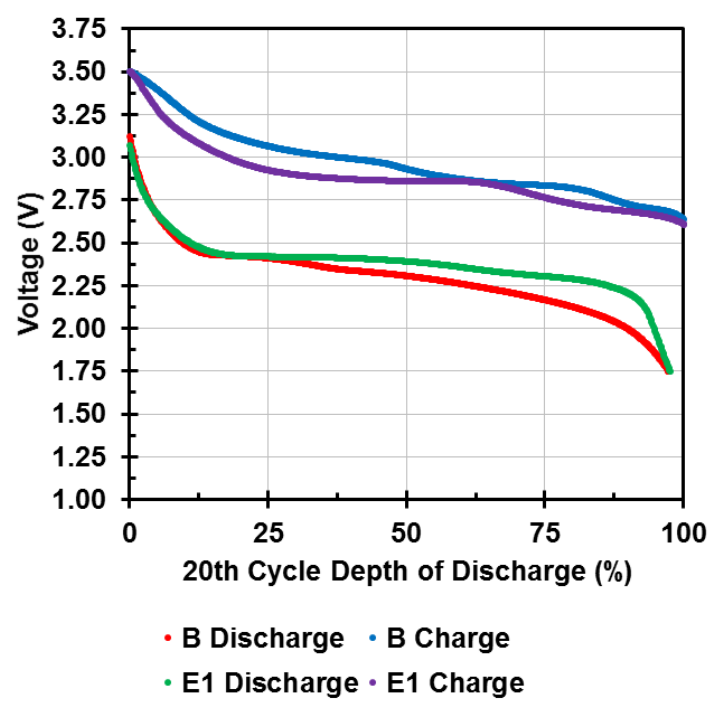

(b)

Figure 4-44: LFP/Cu2Sb Cell Voltage vs. Depth of Discharge (a) $10^{\text {th }}$ Cycle (b) $20^{\text {th }}$ Cycle 
Figure 4-44 shows the voltage of the $\mathrm{LFP} / \mathrm{Cu}_{2} \mathrm{Sb}$ cells as a function of state of charge for the $10^{\text {th }}$ and $20^{\text {th }}$ cycle. The plots were generated by normalizing the measured cell capacity during the $10^{\text {th }}$ and $20^{\text {th }}$ cycle to the total charge capacity of the $10^{\text {th }}$ and $20^{\text {th }}$ cycle, respectively. It can clearly be seen that the E1 cells have a more narrow cycling voltage window at both cycles 10 and 20. This result suggests that the lithium intercalation and de-intercalation kinetics in the active materials are improved for the E1 cells; a consistently lower overpotential is measured over the entire state of charge window compared to B cells. This is a promising result for the proposed E1 electrolyte mixture.

\subsubsection{Lithium Iron Phosphate - Lithium Titanate Oxide Cells}

Figure 4-45 shows the cycling and coulombic efficiency performance of LFP/LTO full cells. Both the E1 and B cells have a first cycle coulombic efficiency of $84 \%$. This value is much higher than the coulombic efficiency seen for the LFP/ $\mathrm{Cu}_{2} \mathrm{Sb}$ cells. At the upper and lower voltage limits of the full cell, minimal active material particle passivation is required, and therefore minimal capacity loss due to
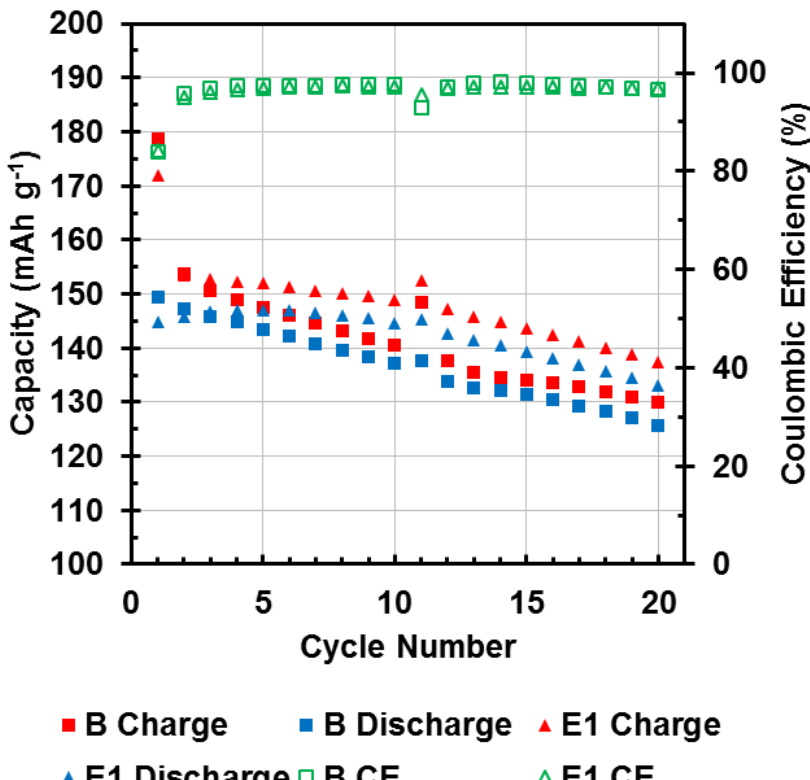

$\triangle$ E1 Discharge $\square \mathrm{BCE} \quad \triangle \mathrm{E} 1 \mathrm{CE}$

Figure 4-45: Charge and Discharge Capacity and Coulombic Efficiency of LFP/LTO Cells lithium consumption in decomposition products is observed on the first cycle. However, over the course of the 20 cycles, significant capacity fade was observed for both E1 and B cells. The rate of capacity fade for both electrolytes is also very similar. This result suggests that despite the two different electrolytes, the same capacity fade mechanism is present in the two cell types. Moreover, 


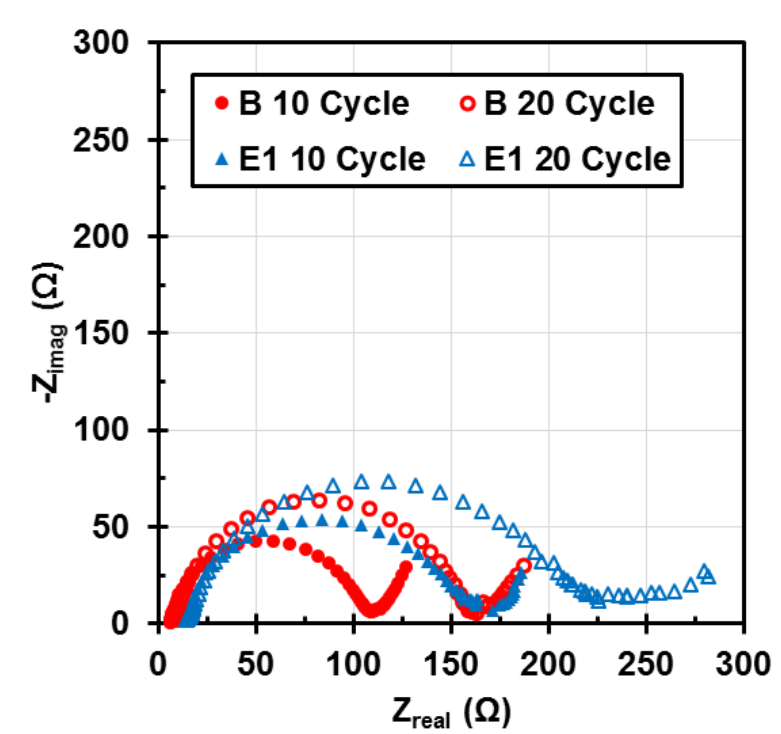

(a)

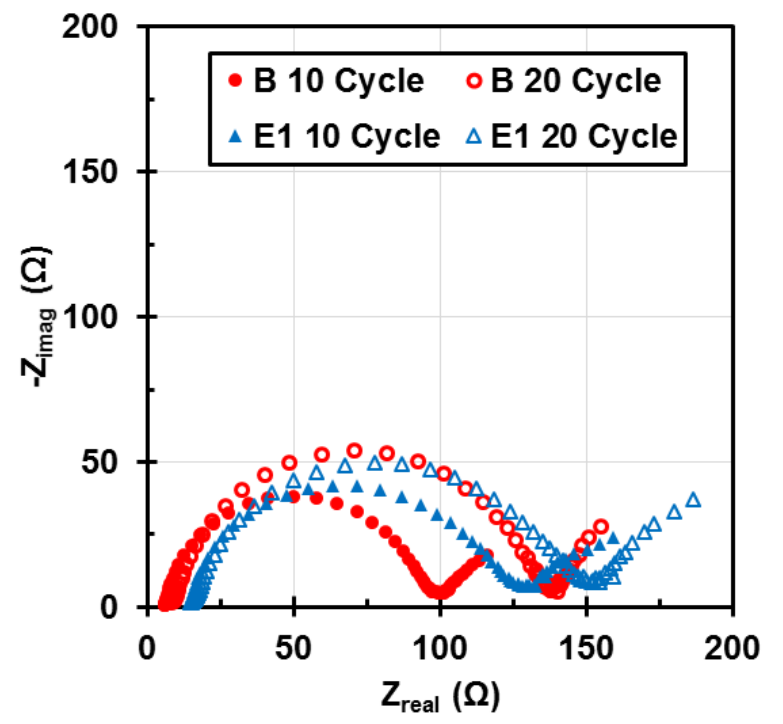

(b)

Figure 4-46: (a) Nyquist Plot at $1.5 \mathrm{~V}$ After 10 and 20 Cycles (b) Nyquist Plot at $2.5 \mathrm{~V}$ After 10 and 20 Cycles for LFP/LTO Cells

the rate of capacity fade is similar to that observed with the B-containing LFP/Li half cells indicating the mechanism could possibly be LFP active material particle cracking.

Figure 4-46a-b shows the cell impedance spectra at the lower and upper voltage limits of the LFP/LTO cells. The cell resistance $\left(R_{\Omega}\right.$ in Table $\left.4-10\right)$ of the B cells increased approximately $18 \%$ from cycles 10 to 20 . The cell resistance for the E1 cells increased as well, but to a much lesser degree $(\sim 5 \%)$. This increase could possibly be due to the LFP particle cracking causing reduced electrical conductivity for the positive electrode. In addition, the charge transfer resistance for both the B and E1 cells significantly increased at both the upper and lower voltage limits. This result indicates that continued decomposition products are formed at the anode and cathode interfaces of the cell increasing the thickness and impedance of the SEI. Figure 4-47 shows the $10^{\text {th }}$ and $20^{\text {th }}$ cycle voltage versus state of charge for the LFP/LTO cells. It can be seen that a larger overpotential is required for the E1 cells compared to B cells for both charge and discharge at the same cycling rate during cycles 10 and 20 . This relates directly to the lower conductivity of the electrolyte and the larger impedances found in the E1 cells compared to the B cells. 


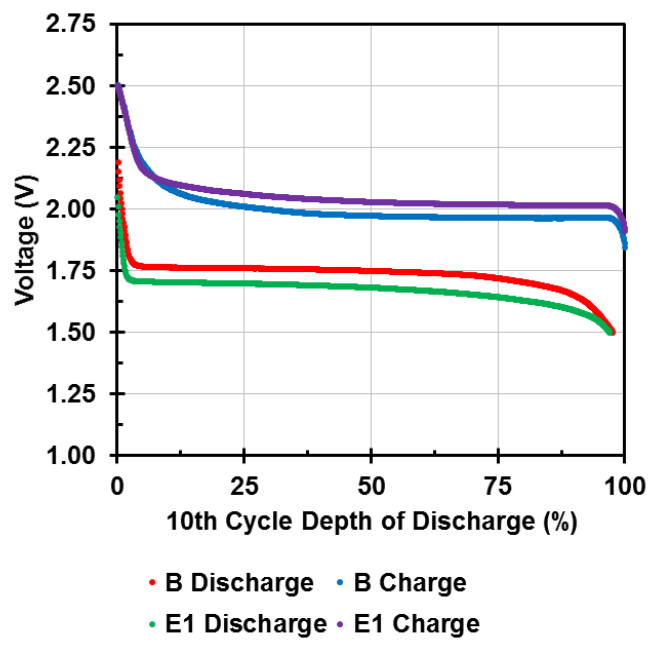

(a)

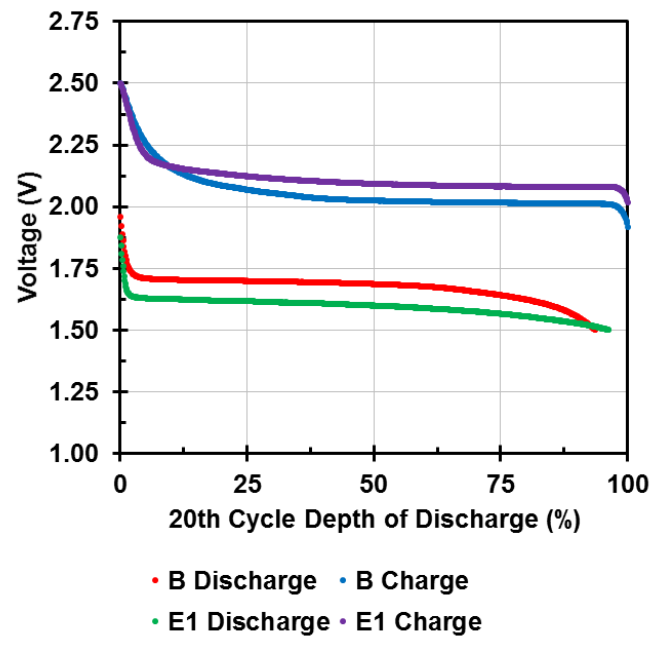

(b)

Figure 4-47: LFP/LTO Cell Voltage vs. Depth of Discharge (a) $10^{\text {th }}$ Cycle (b) $20^{\text {th }}$ Cycle

Table 4-10: Average EIS Fit Parameters for Full Cells

\begin{tabular}{|c|c|c|c|c|c|c|c|c|c|}
\hline \multirow[b]{3}{*}{$\begin{array}{l}\text { Model } \\
\text { Parameter }\end{array}$} & \multirow[b]{3}{*}{ Electrolyte } & \multicolumn{4}{|c|}{$\mathrm{LFP} / \mathrm{Cu} \mathrm{u}_{2} \mathrm{Sb}$} & \multicolumn{4}{|c|}{ LFP/LTO } \\
\hline & & \multicolumn{2}{|c|}{10 Cycle } & \multicolumn{2}{|c|}{20 Cycle } & \multicolumn{2}{|c|}{10 Cycle } & \multicolumn{2}{|c|}{20 Cycle } \\
\hline & & $1.75 \mathrm{~V}$ & $3.5 \mathrm{~V}$ & $1.75 \mathrm{~V}$ & $3.5 \mathrm{~V}$ & $1.5 \mathrm{~V}$ & $2.5 \mathrm{~V}$ & $1.5 \mathrm{~V}$ & $2.5 \mathrm{~V}$ \\
\hline \multirow{2}{*}{$\begin{array}{l}R_{\Omega} \\
{[\Omega]}\end{array}$} & $\mathrm{B}$ & 4.67 & 4.89 & 5.00 & 5.13 & 5.10 & 5.06 & 6.18 & 6.32 \\
\hline & E1 & 12.1 & 12.6 & 12.2 & 13.1 & 12.8 & 13.1 & 13.4 & 14.0 \\
\hline \multirow{2}{*}{$\begin{array}{c}Q \times 10^{4} \\
{\left[\mathrm{~S} \mathrm{~s}^{\alpha}\right]}\end{array}$} & $\mathrm{B}$ & 0.233 & 0.339 & 0.241 & 0.363 & 0.285 & 0.286 & 0.281 & 0.273 \\
\hline & E1 & 0.152 & 0.234 & 0.175 & 0.204 & 0.176 & 0.158 & 0.190 & 0.159 \\
\hline \multirow{2}{*}{$\begin{array}{c}\alpha \\
{[-]}\end{array}$} & $\mathrm{B}$ & 0.823 & 0.783 & 0.819 & 0.776 & 0.826 & 0.826 & 0.815 & 0.818 \\
\hline & E1 & 0.845 & 0.790 & 0.830 & 0.809 & 0.827 & 0.840 & 0.815 & 0.835 \\
\hline \multirow{2}{*}{$\begin{array}{c}W_{\mathrm{d}} \times \mathbf{1 0}^{2} \\
{\left[\mathrm{~S} \mathrm{~s} \mathbf{s}^{1 / 2}\right]}\end{array}$} & $\mathrm{B}$ & 0.135 & 4.71 & 0.148 & 3.93 & 2.54 & 3.52 & 2.39 & 3.10 \\
\hline & E1 & 0.147 & 3.05 & 0.152 & 4.22 & 2.83 & 3.41 & 2.33 & 3.02 \\
\hline \multirow{2}{*}{$\begin{array}{l}R_{\mathrm{ct}} \\
{[\mathbf{\Omega}]}\end{array}$} & $\mathrm{B}$ & 237 & 204 & 318 & 231 & 101 & 89.3 & 141 & 112 \\
\hline & E1 & 141 & 134 & 175 & 115 & 129 & 99.6 & 185 & 128 \\
\hline \multirow{2}{*}{$\begin{array}{c}f_{c} \\
{[\mathrm{~Hz}]}\end{array}$} & $\mathrm{B}$ & 88 & 91 & 61 & 76 & 190 & 219 & 140 & 189 \\
\hline & E1 & 230 & 234 & 170 & 284 & 250 & 344 & 164 & 265 \\
\hline
\end{tabular}




\subsection{Summary}

In this chapter, the non-boiling electrochemical performance of the MFE was investigated. Miscibility testing quickly narrowed the candidate volatile co-solvents for the MFE to HFE-7000 and HFE-7100. FC-72 and Perflenapent were not miscible with EMC and no further testing was performed. HFE-7000 was selected over HFE-7100 for further investigation due to its more favorable thermal properties for the proposed internal TMS. A candidate MFE was formulated using 1.0 M LiTFSI salt in 1:1 HFE-7000/EMC by volume. Room temperature conductivity testing showed that the MFE had a slightly lower ionic conductivity than a baseline electrolyte, $1.0 \mathrm{M}$ $\mathrm{LiPF}_{6}$ in 3:7 EC/DEC by weight.

After these tests, the electrochemical stability window, half and full cell cycling, and impedance spectroscopy measurements were conducted with the MFE and a baseline electrolyte. The electrochemical stability on a glassy carbon electrode showed that the candidate E1 electrolyte had improved oxidative and reductive stability compared to the baseline, and the stability window on a platinum electrode measured impurities in the MFE rather than the stability limits for the electrolyte. In half cell tests with $\mathrm{Cu}_{2} \mathrm{Sb}$ and LTO anode active materials, the candidate electrolyte exceeded the charge-discharge capacity of the baseline electrolyte. Impedance spectroscopy testing showed E1-containing cells had higher cell resistance due to lower ionic conductivity, but in some instances had reduced charge transfer resistance compared to the baseline. Half cell tests with LFP showed the HFE-7000 in the E1 electrolyte is not effective at passivating the aluminum current collector to the LiTFSI salt. Additional refinement to the MFE electrolyte is required to minimize these irreversible reactions. Full cell tests showed that the MFE electrolyte is capable of equally reversible cycling as the baseline electrolyte, with particularly promising performance in $\mathrm{LFP} / \mathrm{Cu}_{2} \mathrm{Sb}$ architecture. 
This study is the first work to investigate HFE-7000 in a lithium-ion electrolyte. Even with its high volatility, the experiments conducted in the present study with HFE-7000 has proven the feasibility of this co-solvent for use in lithium-ion batteries. The thermal and electrochemical performance of the MFE in the proposed internal thermal management system is investigated in Chapter 5. 


\section{CHAPTER 5. ELECTROCHEMICAL PERFORMANCE OF MULTI-FUNCTIONAL ELECTROLYTE WITH SIMULTANEOUS CO-SOLVENT BOILING}

In the previous chapter, the non-boiling electrochemical performance of the MFE was established using a variety of electrochemical experimental techniques. Despite having a lower ionic conductivity and low active material passivation capability, the MFE performed comparably well to the baseline electrolyte. Several electrolyte mixture additives are proposed for the continued improvement of the electrochemical performance of the MFE. However, before further electrolyte refinement is done, the electrochemical performance of candidate MFE needs to be evaluated while the volatile co-solvent is continuously evaporating, which is critical to validating the proposed internal TMS. For validation purposes, the under test will be a LFP/Cu $2 \mathrm{Sb}$ cell, which showed more stable electrochemical performance than the LFP/LTO cells, was selected. To evaluate electrochemical performance while boiling the HFE-7000 co-solvent, a custom electrolyte boiling facility was constructed. The following sections detail the experimental requirements, design, manufacturing, and commissioning of the facility, followed by a description of the testing parameters. Finally, the results and discussion of the electrochemical performance of the MFE under constant vapor generation are presented.

\subsection{Experimental Requirements for Custom Electrolyte Boiling Facility}

The design of the electrolyte boiling facility (EBF) needed to encompass all of the requirements listed in Table 5-1. The EBF must be operated as a closed system to approximate a loop heat pipe architecture and prevent the loss of the volatile HFE-7000 co-solvent during testing. The EBF is a low pressure test facility, and internal pressures greater than $172.4 \mathrm{kPa}$ will be vented. To keep the system pressure low, the MFE is introduced into the EBF while it is under vacuum. In this scenario, the components of the vapor in the EBF are only the components of the liquid 
MFE mixture. This should allow for rapid two-phase heat transfer to occur upon the application of an external heat source. Furthermore, to prevent parasitic side reactions, all of the electrolyte wetted components must be inert to the $\mathrm{LFP} / \mathrm{Cu}_{2} \mathrm{Sb}$ electrodes and the MFE. For safety, all testing with the EBF is done in the argon glove box, and, therefore, the facility must fit in the large antechamber of the glove box. Finally, the EBF must measure relevant temperatures and pressures to evaluate the thermal-hydraulic performance of the MFE. In the next section, the design of the facility is presented.

\section{Table 5-1: Requirements of the Electrolyte Boiling Facility}

\section{\# Requirement}

1 The EBF must be a closed system constructed with non-reactive materials.

2 The electrolyte wetted EBF components must be chemically clean.

3 The EBF must withstand internal pressures up to $172.4 \mathrm{kPa}$ (25 psig).

4 The EBF must contain and electrically connect to a positive electrode with an electrolyte evaporation channel and a negative electrode.

5 The EBF must be capable of variably applying heat to the positive electrode to induce boiling in the evaporation channel.

6 The EBF must allow for direct viewing of vapor generation in the positive electrode channel.

7 The EBF must accurately measure the bulk electrolyte temperature, positive electrode temperature, and condenser inlet and outlet temperatures.

8 The EBF must accurately measure the electrolyte vapor pressure.

9 The EBF must interface with a battery testing instrument (potentiostat) to cycle the cell.

10 The EBF must measure the voltage of the cell independent of the potentiostat.

11 The EBF electrolyte wetted test section must be capable of complete vacuum evacuation and charging with electrolyte.

12 The EBF must fit into the large glove box antechamber and be capable of assembly in glove box.

13 The EBF must be fully capable of disassembly for cleaning and component replacement, if necessary.

\subsection{Electrolyte Boiling Facility}

In this section, the design, fabrication, and assembly of and the cleaning and filling procedures for the boiling facility are documented. When reviewing the design, the material 
selection and compatibility issues for all components are discussed. Some of the components are not compatible with the battery materials, and, as a result, are isolated from the MFE and electrode assembly. The items in contact with the electrolyte are thoroughly cleaned to prevent impurities from impacting the results. This includes the electrolyte, which also must be a pure fluid mixture and special filling procedures are utilized

\subsubsection{Facility Design and Fabrication}

The design of the EBF is given in Figure 5-1 and Figure 5-2. As shown in Figure 5-1, two 316 stainless steel ball valves are used for system evacuation and charging of the MFE. A 172.4 $\mathrm{kPa}$ pop safety valve is used to ensure that the system is never pressurized above this value. The test section contains a small $\mathrm{LFP} / \mathrm{Cu}_{2} \mathrm{Sb}$ cell contained within a sight glass. The surrounding infrastructure of the test facility mimics a closed-loop thermosiphon. All generated vapor is routed out of the test section through $6.35 \mathrm{~mm} 316$ stainless steel smooth-bore tubing and into a shell-andtube water-cooled condenser that is connected to the test

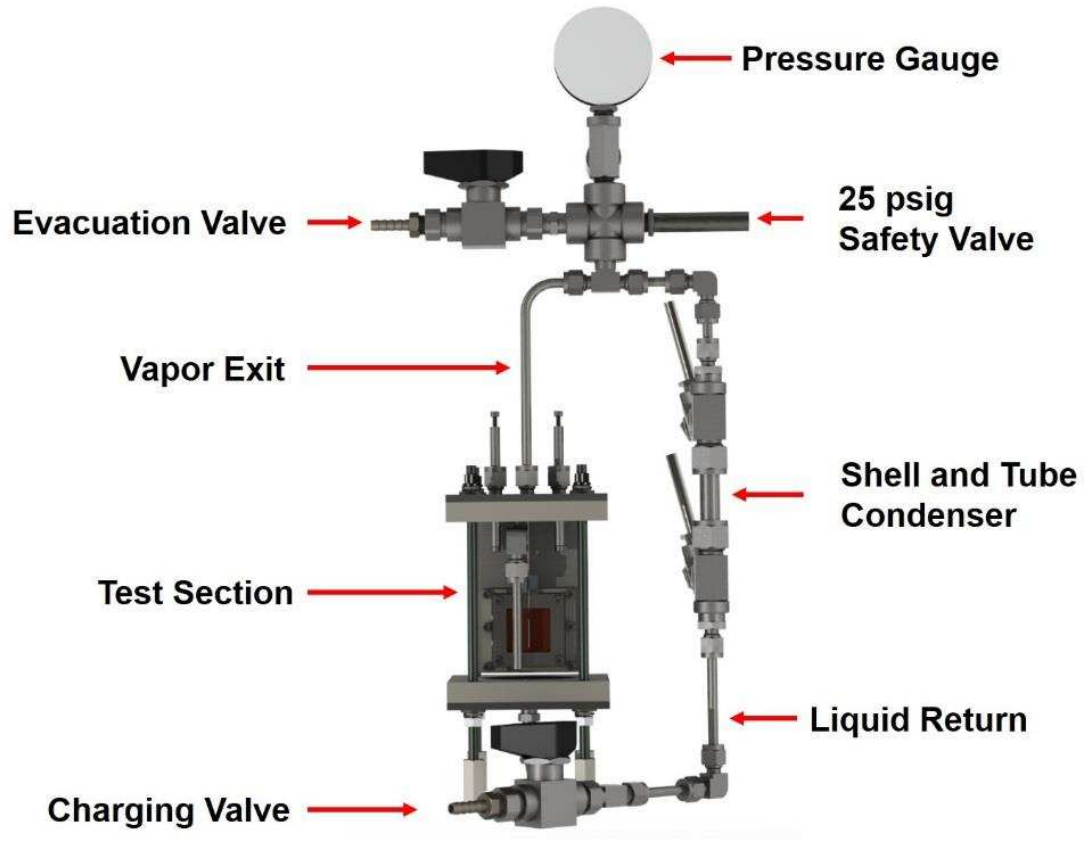
section.

\section{Figure 5-1: Electrolyte Boiling Facility}

An exploded view of the test section is provided in Figure 5-2. A $10 \mathrm{~W}$ thin film heater $(25.4 \mathrm{~mm} \times 25.4 \mathrm{~mm})$ interfaces with the backside of the LFP electrode, which contains a single evaporation channel in the center of the active material coating. The thin film heater simulates 


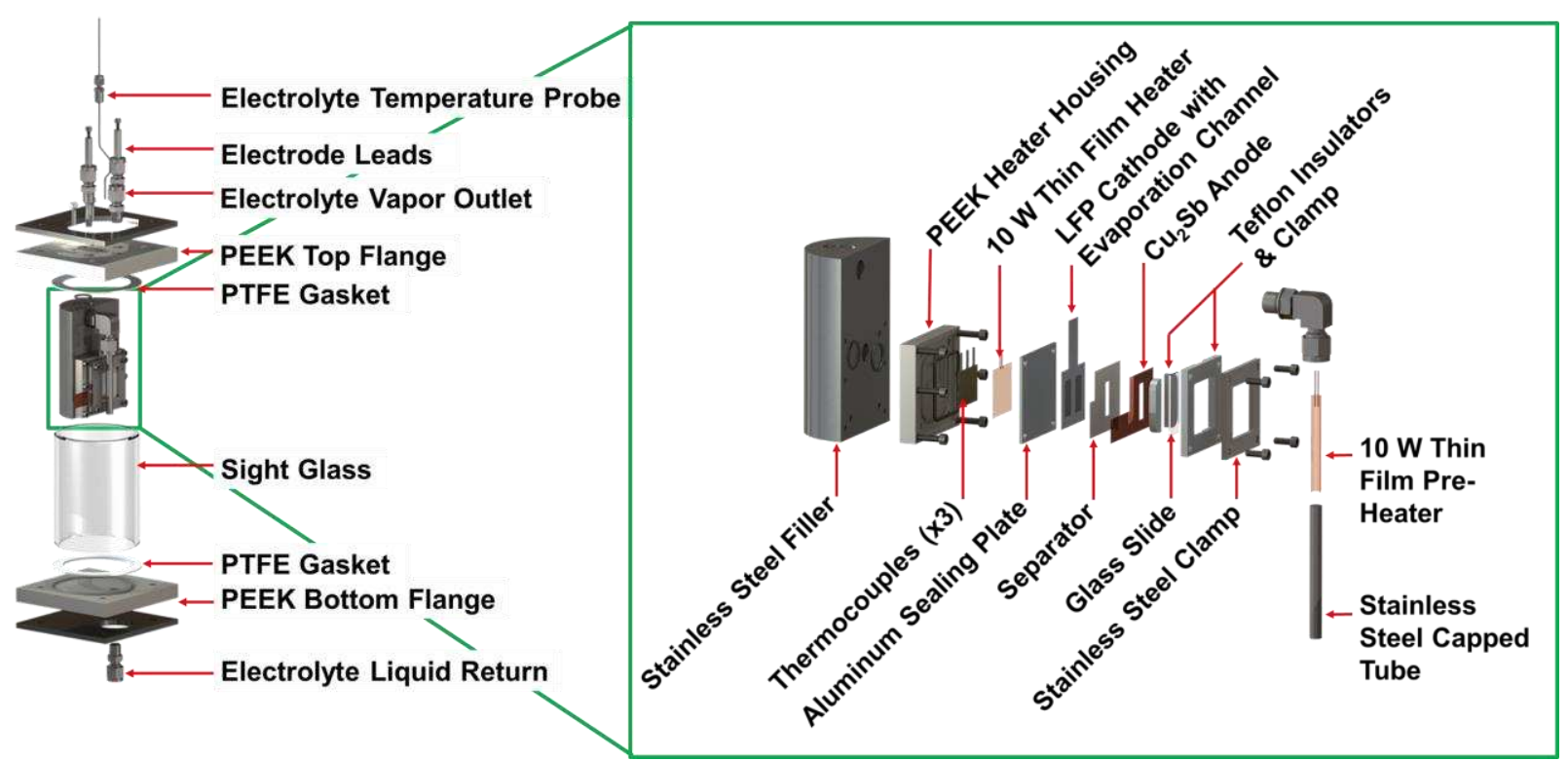

Figure 5-2: Electrolyte Boiling Facility: Exploded View of Test Section

extreme heat generation in a LIB to continuously generate vapor in the LFP electrode channel while the cell is cycled. It is important to note that the applied heat is not representative of normal heat generation in a LIB, which is typically on the order of $100 \mathrm{~W} \mathrm{~L}^{-1}$ [3], but is intended to represent thermal runaway of neighboring cells. The $10 \mathrm{~W}$ heater is capable of applying up to 1.55 $\mathrm{W} \mathrm{cm}{ }^{-2}$ of heat to the backside of the positive electrode of the cell; normal heat generation of 100 $\mathrm{W} \mathrm{L} \mathrm{L}^{-1}$ scales to approximately $136 \mu \mathrm{W} \mathrm{cm}{ }^{-2}$. Further discussion on the normal heat generation of a LIB is given in Section 5.4. The material incompatibility of the $10 \mathrm{~W}$ thin film heater and thermocouples in the MFE required it to be isolated from the cell. To do this, a PEEK heater housing and stainless steel filler were used. The PEEK heater housing interfaces with an aluminum sealing plate to keep the heater and thermocouples sealed from the MFE. The stainless steel filler provides a mounting location for the heater housing, a wire passage for the heater and thermocouples, and substantially reduces the volume of MFE required to completely submerge the $\mathrm{LFP} / \mathrm{Cu}_{2} \mathrm{Sb}$ cell. The separator and $\mathrm{Cu}_{2} \mathrm{Sb}$ anode contain a window that enable direct viewing of the vapor generation channel in the LFP electrode. 
A full list of the components used to construct the EBF is provided in Appendix D. The component description, supplier, and part number are also provided. Particular attention was given to the selection of electrolytewetted materials used in the facility. Very minimal information

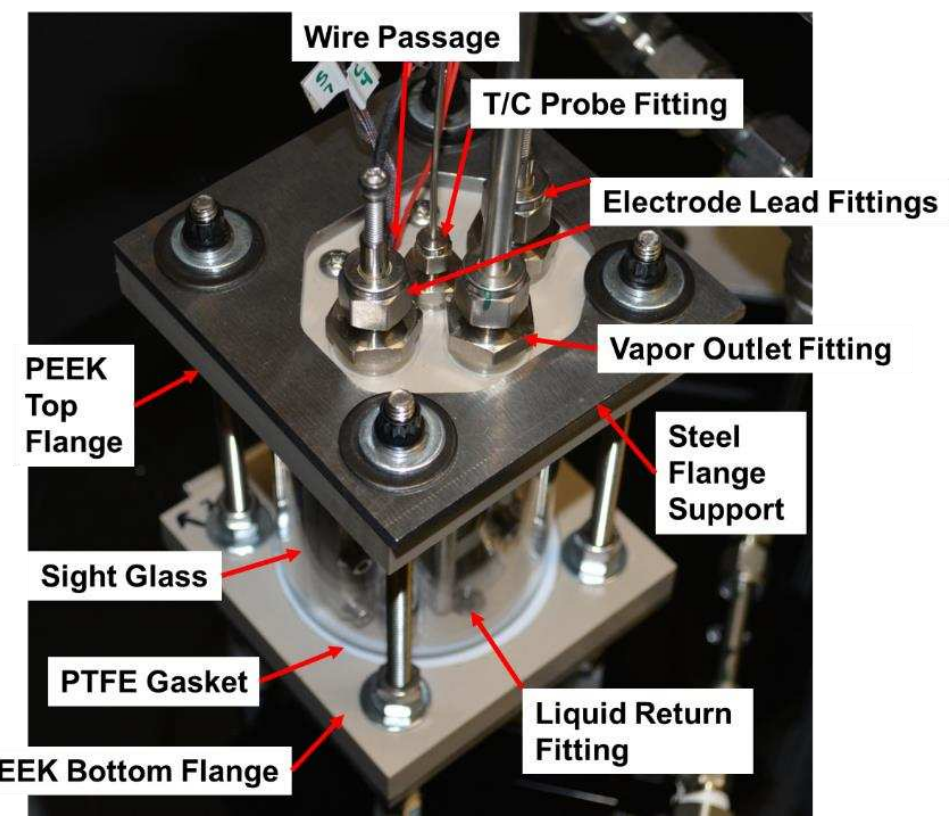

Figure 5-3: Interface of PEEK Top and Bottom Flanges is available on material with Test Section compatibility for lithium-ion electrolytes, especially for a novel MFE mixture; however, there are materials that are consistently used in LIB research and manufacturing. Following this logic, the only wetted materials of the EBF are 316 stainless steel, 3003 aluminum, unfilled PEEK plastic, PTFE Teflon, and Parker compound FF500-75 (used for all o-rings). All o-ring grooves were designed using Parker inPHorm software available online.

As seen in Figure 5-3, the PEEK top and bottom flanges provided grooves for PTFE gaskets to seal the glass sight. In addition, the top flange located four Swagelok fittings: two for electrical connections with the LFP and $\mathrm{Cu}_{2} \mathrm{Sb}$ electrodes, one for a vapor exit, and one for a thermocouple probe. Furthermore, the top flange also interfaced with the stainless steel filler,
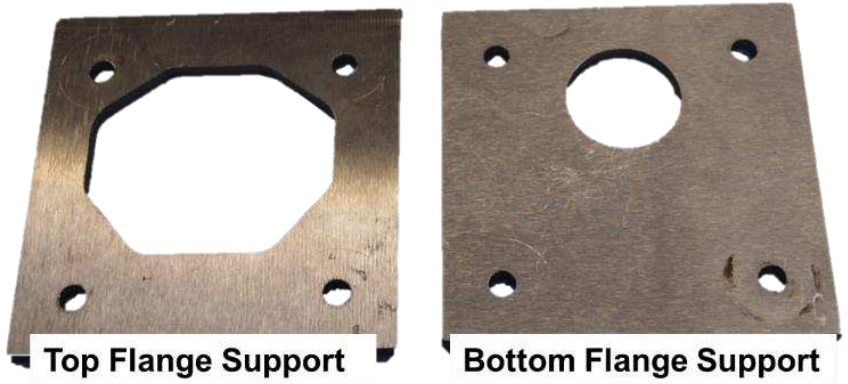

providing an o-ring seal for the heater and thermocouple wire passage. The PEEK bottom flange interfaced with the Swagelok liquid return fitting. The Figure 5-4: Steel Top and Bottom Flange Supports 
steel top and bottom flange supports

(Figure 5-4) behaved like washers, spreading the clamping load created by the four $1 / 4-20$ threaded rods more evenly across the PEEK top and bottom flanges.

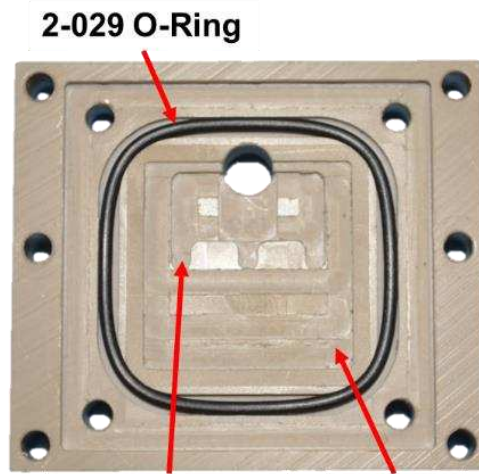

Thermocouple Heater Recess
2-013 O-Ring

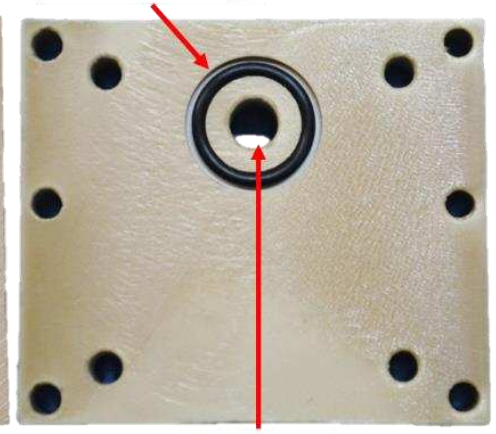

Wire

Passage

The PEEK heater housing is Figure 5-5: PEEK Heater Housing with O-Rings

seen in Figure 5-5 with its two FF500-75 o-rings. A small recessed pocket (0.254 mm depth) was created for the $10 \mathrm{~W}$ thin film heater. Additional recesses were machined for the three surface thermocouples placed on the backside of the thin film heater (Figure 5-6). Four \#8 close fit clearance holes were drilled to allow for the 8-32 screws to pass through and provide clamping force on the 2-029 o-ring seal. In addition, six \#6 close fit clearance holes were drilled for 6-32 screws to pass through and mount the PEEK heater housing to the stainless steel filler and provide clamping force for the 2-013 o-ring seal on the backside of the part.

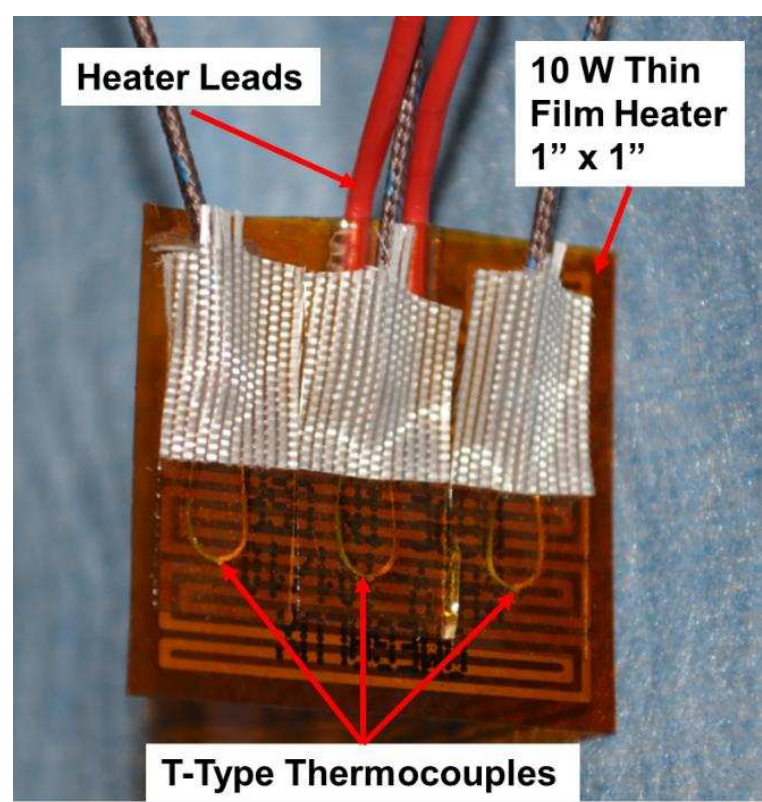

Figure 5-6: $10 \mathrm{~W}$ Thin Film Heater with Three T-Type Surface Thermocouples
The stainless steel filler and relevant features are highlighted in Figure 5-7. Most critically, the stainless steel filler provides a sealed heater and thermocouple wire passage. The stainless steel filler interfaces with the PEEK top flange with two 8-32 screws which provide clamping force on a 2-013 o-ring seal. It also provides the mounting location for the PEEK heater housing and entire stacking assembly seen 


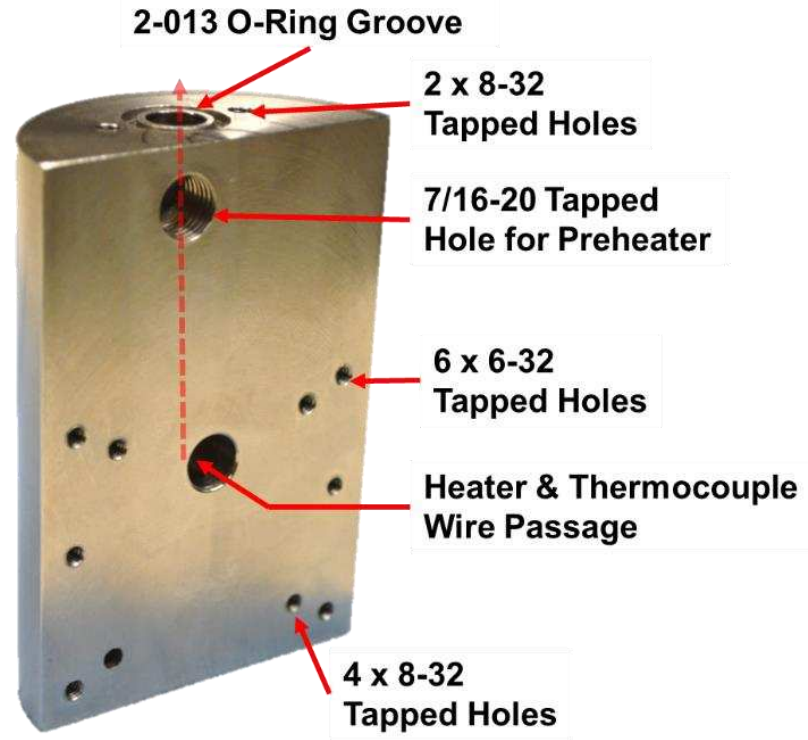

Figure 5-7: 316 Stainless Steel Filler in Figure 5-2. Moreover, the stainless steel filler contains a mounting location for a preheater, also seen in the exploded view of the test section (Figure 5-2). The preheater contains a $10 \mathrm{~W}$ thin film heater $(12.7 \mathrm{~mm}$ $\times 50.8 \mathrm{~mm}$ ) and is used to preheat the fluid to near the saturation temperature. Figure 5-8 shows the how the preheater wire passage connects with the main sealed wire

passage.

The LFP electrode contains two rectangular patches $(19.1 \times 7.62 \mathrm{~mm})$ of active material separated by $6.35 \mathrm{~mm}$ to create a vapor generation channel (Figure 5-9). The LFP electrode slurry was the same composition as the slurry used for half and full cell experiments (Table 4-4). The LFP slurry was applied to a $50 \mu \mathrm{m}$ aluminum foil cut to its final shape and vacuum dried overnight. Excess active material was removed with a metal spatula. The electrode was then calendared to a total thickness of $93 \mu \mathrm{m}$ with an active material weight of $0.0225 \mathrm{~g}$. The $\mathrm{Cu}_{2} \mathrm{Sb}$ electrode was fabricated by direct electrodeposition onto $25 \mu \mathrm{m}$ copper foil using process described elsewhere

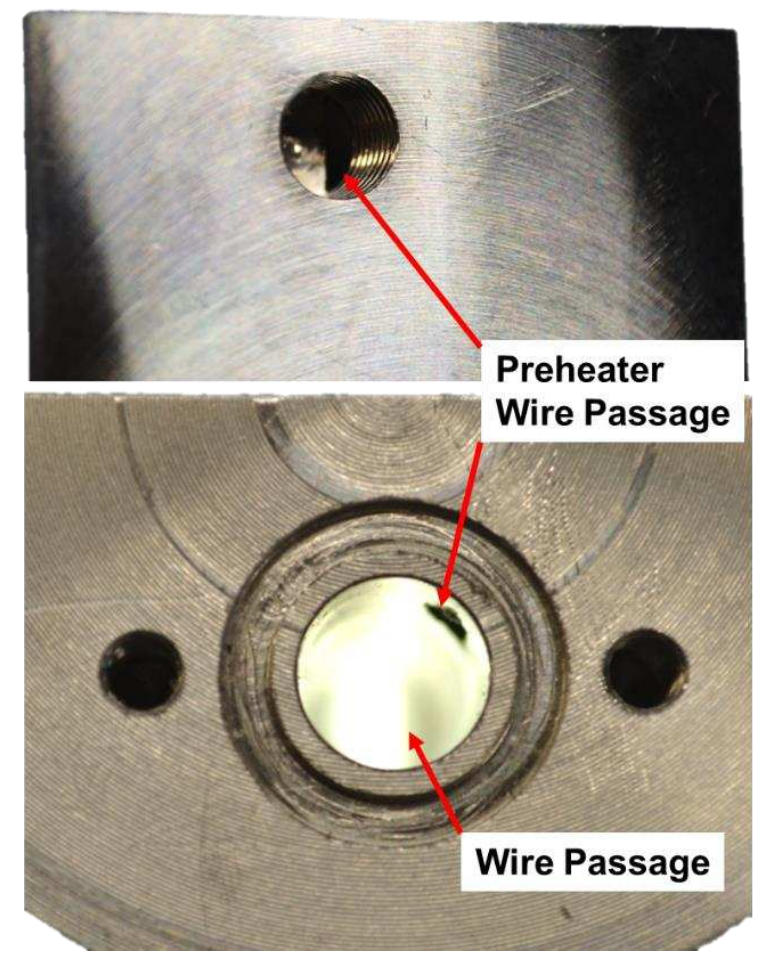

Figure 5-8: Preheater Wire Passage in Stainless Steel Filler 


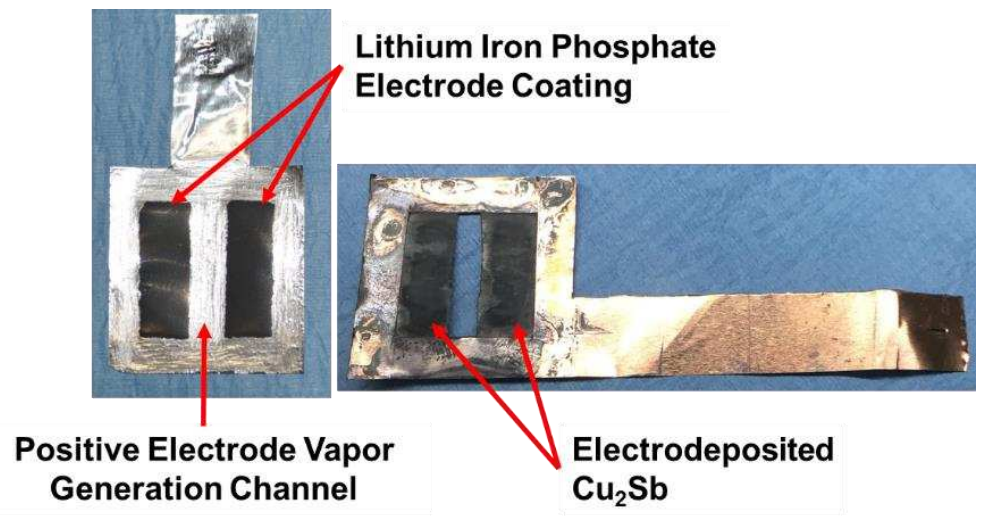

Figure 5-9: $\mathrm{LFP}$ and $\mathrm{Cu}_{2} \mathrm{Sb}$ Electrodes Used in Electrolyte Boiling Facility
[127]. Prior to electrodeposition, a $19.1 \times 5.6 \mathrm{~mm}$ window was cut into the center of the copper foil to allow for direct viewing of the evaporation channel on the LFP electrode (Figure 5-9). Kapton tape was used to mask all submerged portions of the copper foil in the deposition electrolyte except for two locations of the same approximate area of $19.1 \times 7.62 \mathrm{~mm}$ on either side of the window, where $3 \mu \mathrm{m}$ of $\mathrm{Cu}_{2} \mathrm{Sb}$ was deposited. The $\mathrm{Cu}_{2} \mathrm{Sb}$ electrode contained approximately $0.0130 \mathrm{~g}$ of active material. A $31.8 \mathrm{~mm}$ tall $\times 11.1 \mathrm{~mm}$ wide $\times 1 \mathrm{~mm}$ thick piece of borosilicate glass was placed on the backside of the $\mathrm{Cu}_{2} \mathrm{Sb}$ electrode to confine any nucleate boiling to the evaporation channel (Figure 5-10). A 25 $\mu \mathrm{m}$ polypropylene/polyethylene separator (MTI) was cut to ensure no electrical shorting between the anode and cathode and obstruction of the LFP evaporation channel.

Prior to assembling the facility, all parts wetted by the electrolyte were thoroughly cleaned. In the next two sections, the cleaning procedure and assembly of the facility are described.
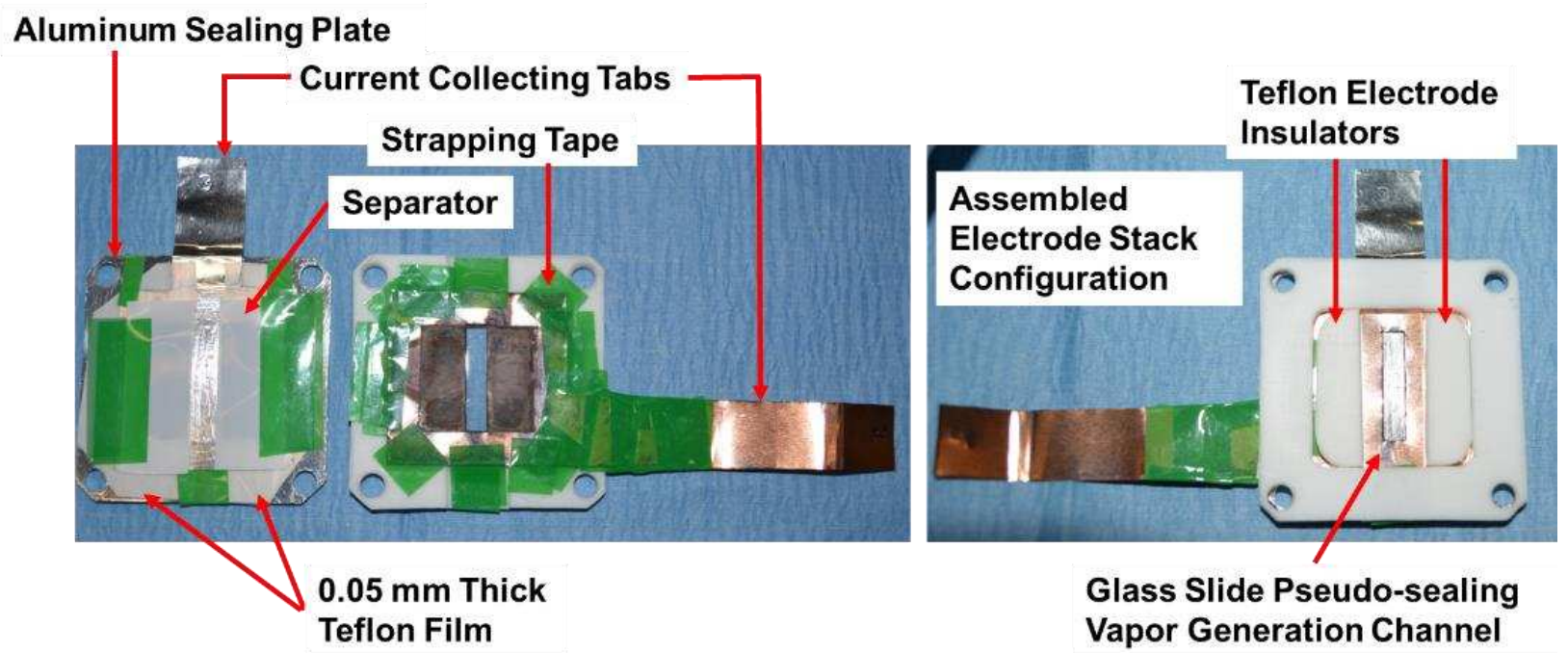

Figure 5-10: Electrode Configuration in Electrolyte Boiling Facility 


\subsubsection{Cleaning of Electrolyte Wetted Components}

Cleaning the electrolyte wetted components - except the LFP and $\mathrm{Cu}_{2} \mathrm{Sb}$ electrodes, which were only vacuum dried - in the EBF was the most labor-intensive task of the experimental setup. Cleaning required the use of nitrile gloves for handing of all components. All electrolyte-wetted metallic components except for tubing were hand polished with Wenol metal polish. The metal polish was applied to a Kimwipe and rubbed on all of the surfaces of the metallic component, which would initially darken the metal surface. The surface was then cleaned with a fresh Kimwipe, which caused the metal surface to have a substantially brighter appearance. All components (metallic and non-

metallic) were then cleaned with the following solvents in the specified order: de-ionized water, acetone, methanol, and isopropanol (Figure 5-11). Finally, all components were vacuum dried overnight at $80^{\circ} \mathrm{C}$ before final assembly both inside

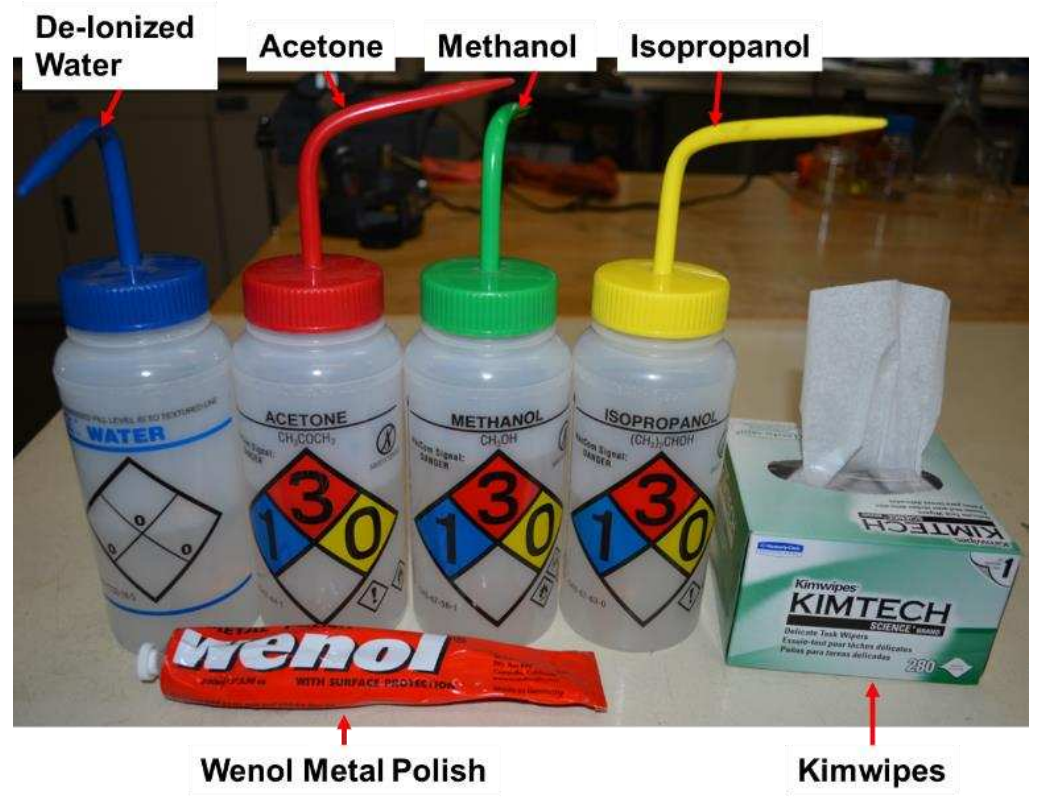
and outside of an argon glove box.

Figure 5-11: Metal Polish and Solvents Used for Cleaning Electrolyte Wetted Components 


\subsubsection{Assembly of Electrolyte Boiling Facility}

The assembly of the EBF was conducted in two phases: outside and inside the argon glove box. The test section was assembled outside of the glove box first. This portion of the assembly included all components seen in Figure 5-2 and was performed with a clean pair of nitrile gloves. To help with positioning the electrodes and separator in the stacked electrochemical cell in the test section, green strapping tape $(10 \mathrm{~mm}$ wide $\times 0.03 \mathrm{~mm}$ thick, MTI) was used (Figure 5-10). The strapping tape located the LFP positive electrode on the aluminum sealing plate and the $\mathrm{Cu}_{2} \mathrm{Sb}$ negative electrode on the Teflon clamp. Prior to locating the $\mathrm{Cu}_{2} \mathrm{Sb}$ electrode on the Teflon clamp with tape, the glass slide was positioned into the groove on the Teflon clamp. To supplement the electrical insulation between the two electrodes provided by the separator, a $0.05 \mathrm{~mm}$ thick Teflon sheet was positioned around the edge of the positive electrode to create additional physical separation of the LFP and $\mathrm{Cu}_{2} \mathrm{Sb}$ electrodes. With this arrangement, the compressive load applied

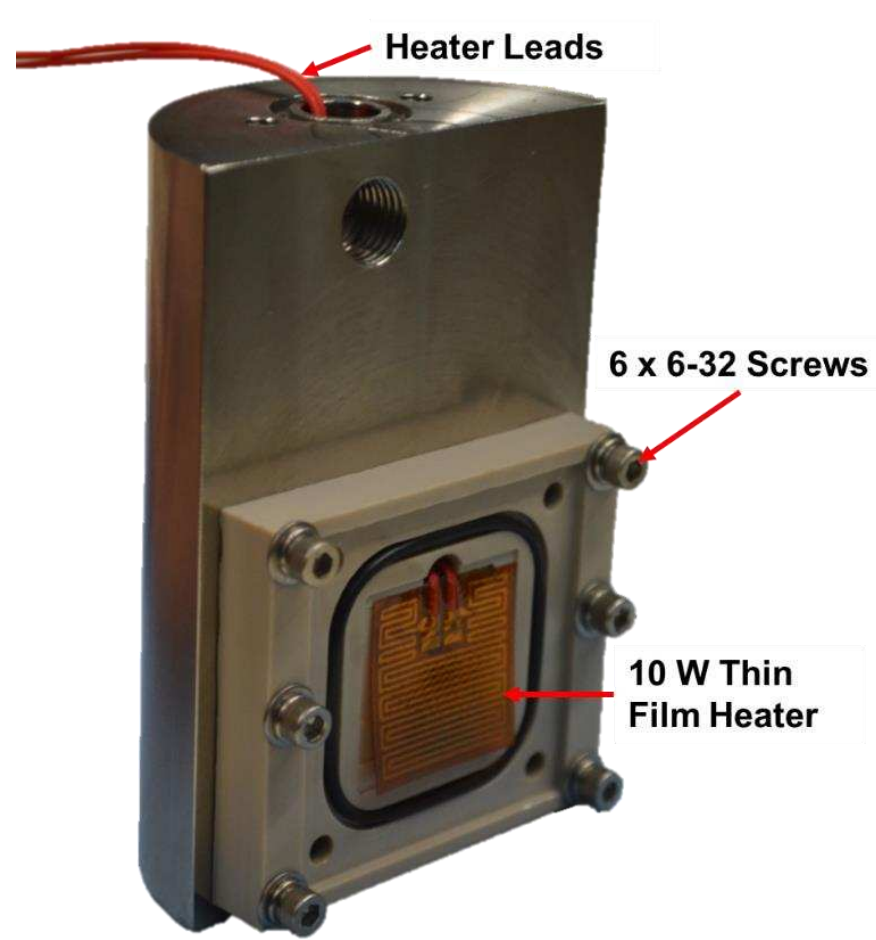

Figure 5-12: Mounting of PEEK Heater Housing flange. The PEEK heater housing was then onto Stainless Steel Filler with 10 W Thin Film Heater to the cell stacking architecture would not compress and compromise the porous separator. The addition of the Teflon sheet increased the spacing between the LFP and $\mathrm{Cu}_{2} \mathrm{Sb}$ electrode to approximately $50 \mu \mathrm{m}$. After the electrodes and separator were located with strapping tape, the thin film heater wires and thermocouple wires were carefully fed through the PEEK heater housing, stainless steel filler, and PEEK top 
secured to the stainless steel filler using six 6-32 screws (Figure 5-12). The aluminum sealing plate was placed on top of the heater over the 2-029 o-ring such that the vapor channel is visible through the glass. The four 8-32 screws were tightened into the stainless steel filler to create the clamping force necessary to compress the heater oring. The wires of the preheater were then fed through the stainless steel filler wire passage shown in Figure 5-8. The o-ring

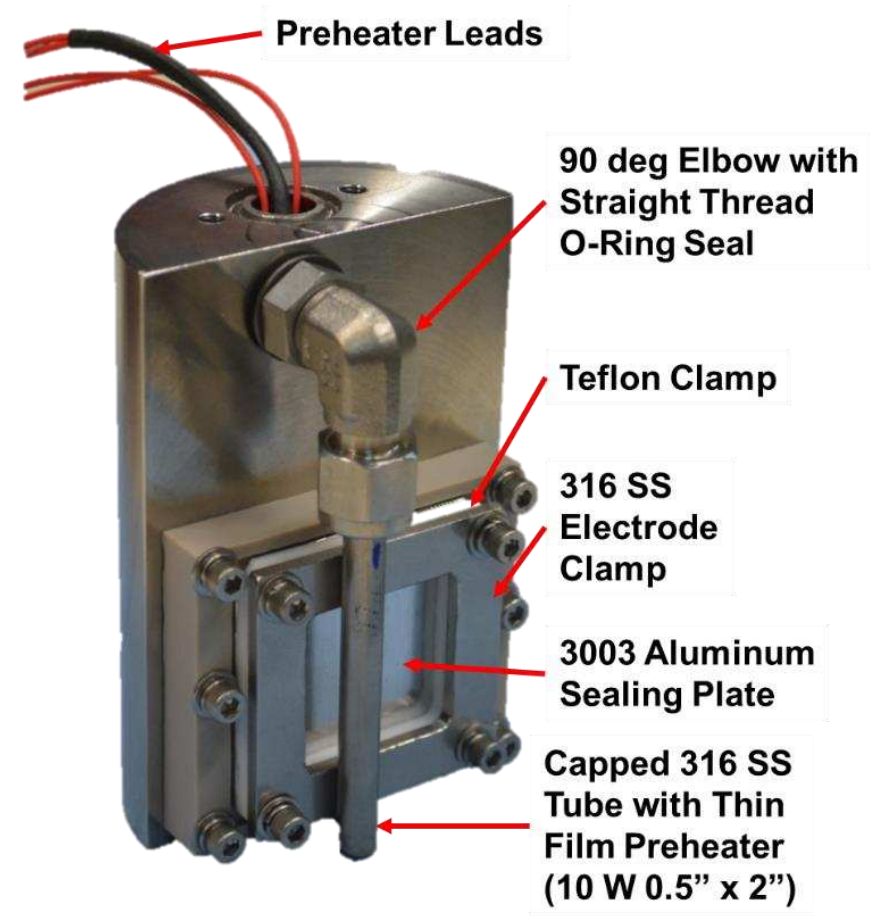

Figure 5-13: Assembled Test Section with Preheater and without Electrodes

elbow fitting of the preheater was then tightened to form a seal on the face of the stainless steel filler. Figure 5-13 shows the assembled test section with the preheater and without the LFP and

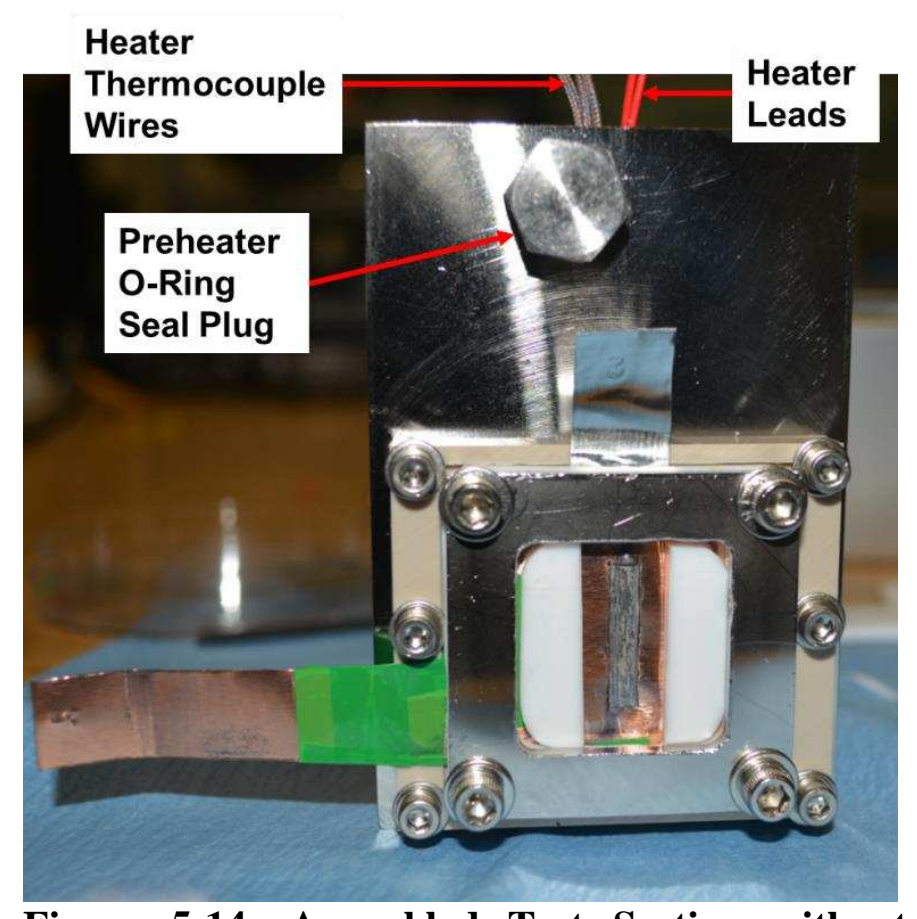

Figure 5-14: Assembled Test Section without Preheater and with Electrodes
$\mathrm{Cu}_{2} \mathrm{Sb}$ electrodes, and Figure 5-14 shows the assembled test section without the preheater and with the electrodes.

The PTFE gasket was inserted into its machined groove in the PEEK top flange. The top flange was then attached to the stainless steel filler using two 8-32 screws with an o-ring sealing head (Figure 5-15 and Figure 5-16). These two screws also provided the necessary compression 


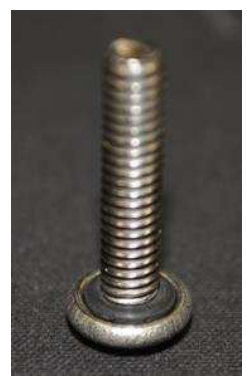

force for the o-ring seal on the top of the stainless steel filler. The fittings shown in Figure 5-16 were tightened onto the top surface of the top flange. The positive and negative electrode electrical connections were then created with 316 stainless steel wire and smooth jaw stainless steel Figure 5-15: 8-32 Screw with O-Ring Sealing Head

alligator clips (Figure 5-17). One end of the connection wire was wrapped around the screw connected to the electrode leads and the other was placed in between the alligator clip and the current collecting tabs of the electrodes. During assembly, a digital multimeter was used to measure the resistance between the electrode leads; if the test section was assembled properly, infinite resistance Gasket

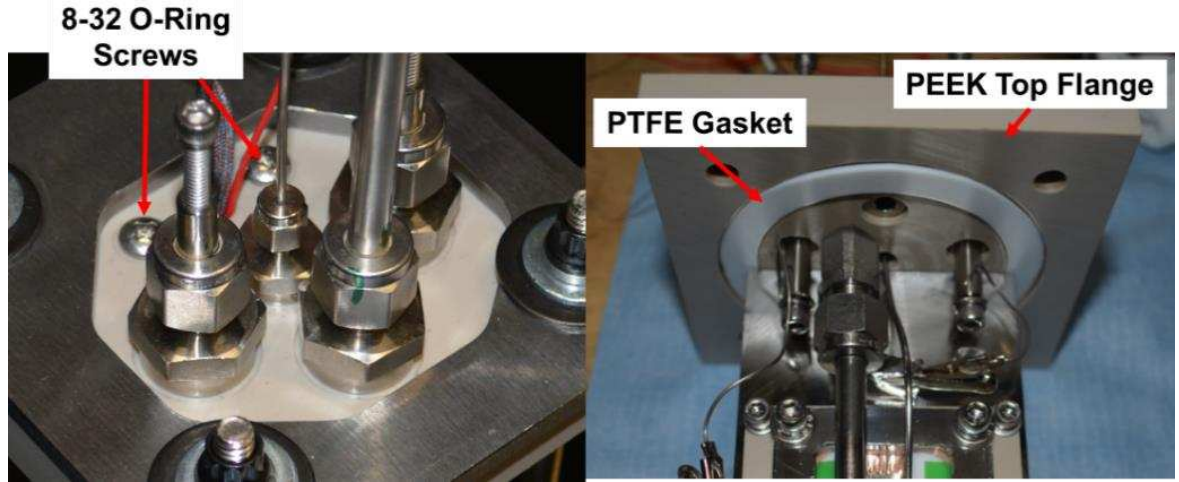

Figure 5-16: Installed 8-32 O-Ring Screws, Fittings, and PTFE should be measured the positive and negative electrodes before it was submerged in the electrolyte.

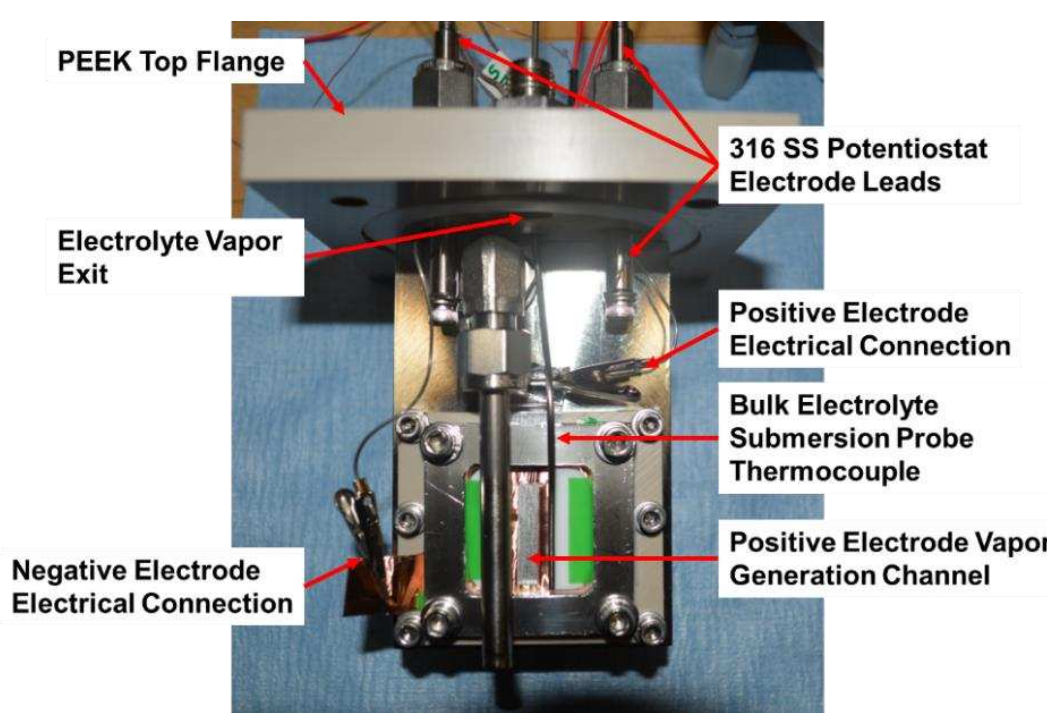

Figure 5-17: Assembly of the Test Section with Top Flange and Electrode Leads
Part of the design challenge of the boiling facility was ensuring all components fit into the cylindrical glass sight and minimized the volumetric fill of the MFE to reduce cost (Section 4.1.1). Figure 5-18 shows a bottom view of the 


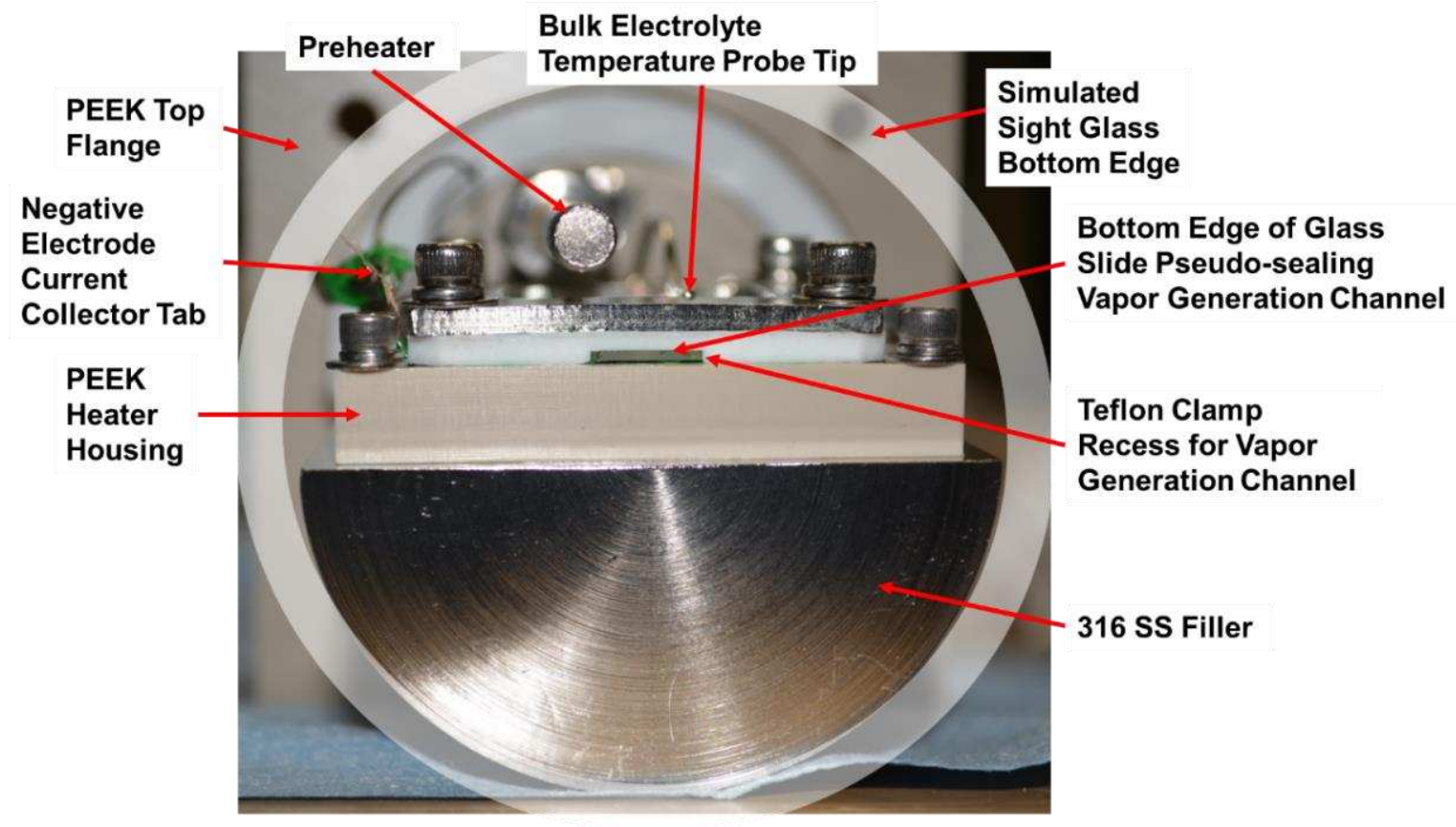

Figure 5-18: Bottom View of Assembled Test Section with Top Flange and Electrode Leads with Simulated Sight Glass Bottom Edge

assembled test section with the approximated clearances of the glass sight. All of the components fit snugly into the $66.8 \mathrm{~mm}$ inner diameter of the sight glass. Special care was taken when inserting the stainless steel filler and assembled electrode stack into the sight glass. The electrode lead wires and current collector tabs needed to be carefully folded inwards to ensure they did not catch on the glass sight edge. The sight glass and top flange/stainless steel filler assembly were then aligned with the bottom flange by the four 1/4-20 threaded rods. High torque 12-point flange nuts were used to provide the clamping force of the glass sight on the PTFE gaskets via the threaded rods. Figure 5-19 shows the final assembled test section.

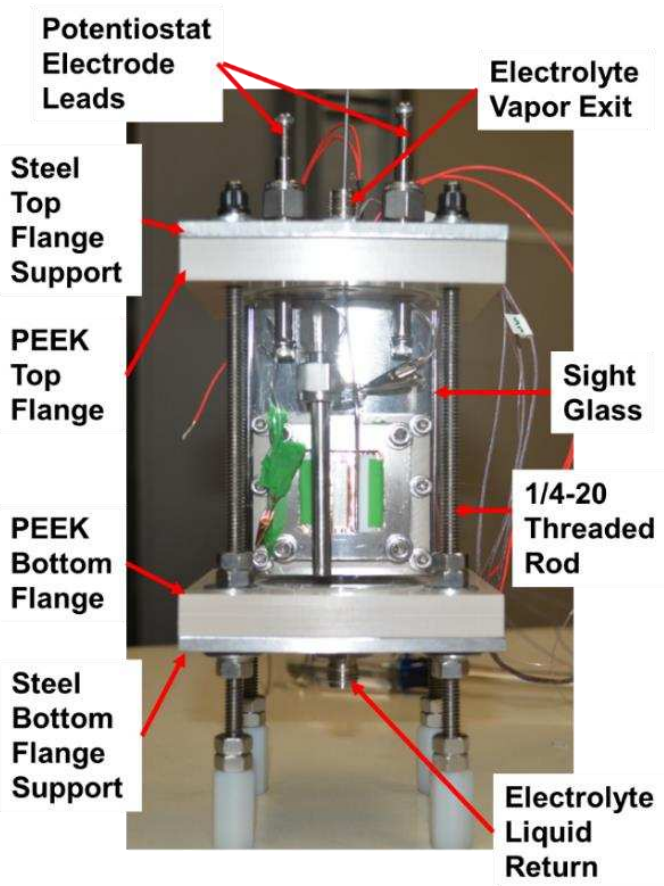

Figure 5-19: Final Assembly of Test Section with Sight Glass and Bottom Flange 
Upon completion of the test section, all components were placed into the glove box for the second phase of the assembly. The components were first placed in a large antechamber, which was evacuated and filled with Argon for a total of three cycles (Figure 5-20). The atmosphere of the large antechamber was evacuated for a minimum of 30 minutes for each cycle. At the conclusion of the atmosphere exchange, the water and oxygen levels introduced by the lab atmosphere have been reduced to levels acceptable for the argon glove box environment.

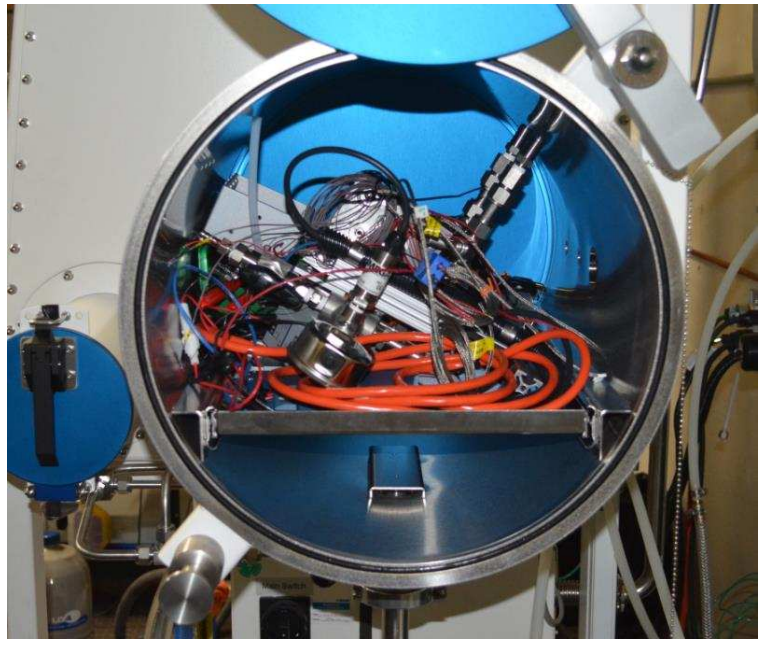

Figure 5-20: Inserting all Electrolyte Boiling Facility Components into Glove Box via the Large Antechamber
Inside the glove box, the test section was connected to the surrounding loop heat pipe structure; the condenser water lines were connected to the re-circulating chiller; all temperature, pressure and voltage leads were wired and connected to the data acquisition system; and the electrodes leads were connected to a potentiostat (Gamry, used for all EBF experiments, Figure 4-8).

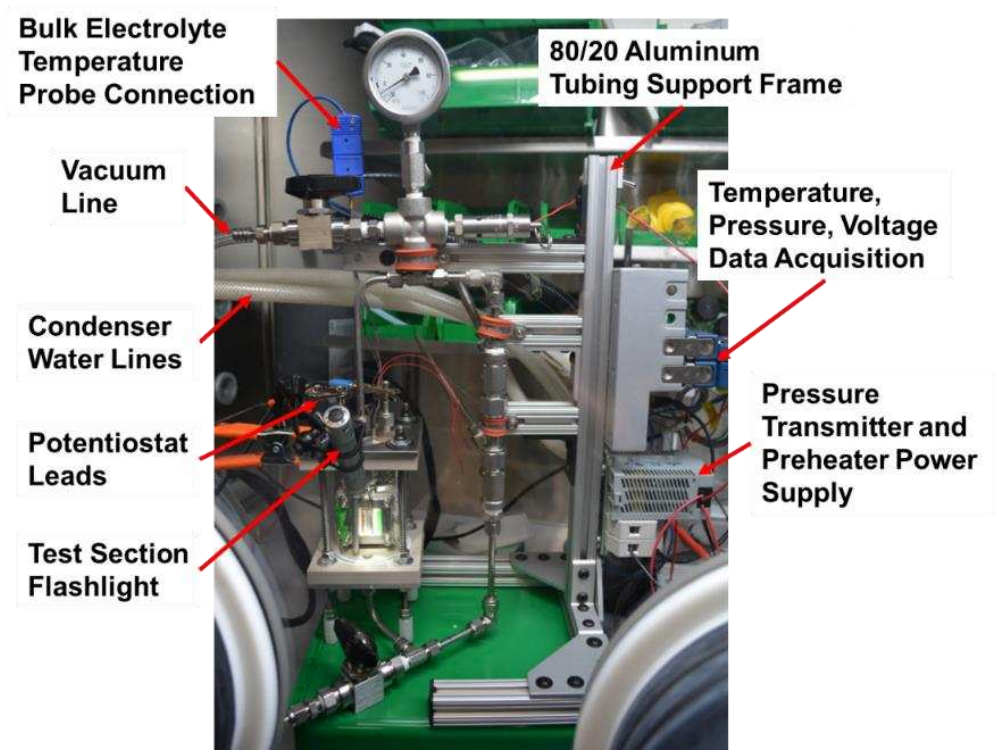

Figure 5-21: Fully Assembled Electrolyte Boiling Facility in Glove Box 
The fully assembled EBF in the glove box is seen in Figure 5-21. Further details on the EBF integration with auxiliary equipment are discussed in Section 5.3. The procedure used to fill the test section with electrolyte is described in the next section.

\subsubsection{Charging Electrolyte Boiling Facility with Multi-Functional Electrolyte}

To ensure that the only components of the vapor in the EBF were constituents of the MFE mixture, a charging process for the system was developed where all of the gas trapped within the facility is evacuated prior to introducing only the liquid MFE. The process of charging the EBF with the MFE was developed using water outside the glove box prior to the cleaning and assembly process. The charging process required the modification of a $180 \mathrm{~mL}$ glass jar that was used for the initial MFE mixing. A cap that fit the jar was modified to contain a $6.35 \mathrm{~mm}$ ID Teflon dip tube that extends to the bottom surface of the glass jar (Figure 5-22). The Teflon dip tube slides snugly over an epoxied $6.35 \mathrm{~mm}$ OD 316 stainless steel tube that is inserted through the cap. Because the Teflon tube extended to the bottom of the jar, only the liquid phase of the electrolyte will be drawn into the boiling facility if the jar was maintained in an upright position. The modified jar cap also contained a small check valve with a $13.4 \mathrm{kPa}$ (2 psi) cracking pressure to allow the pressure within the jar to equalize as the MFE is drawn from it.

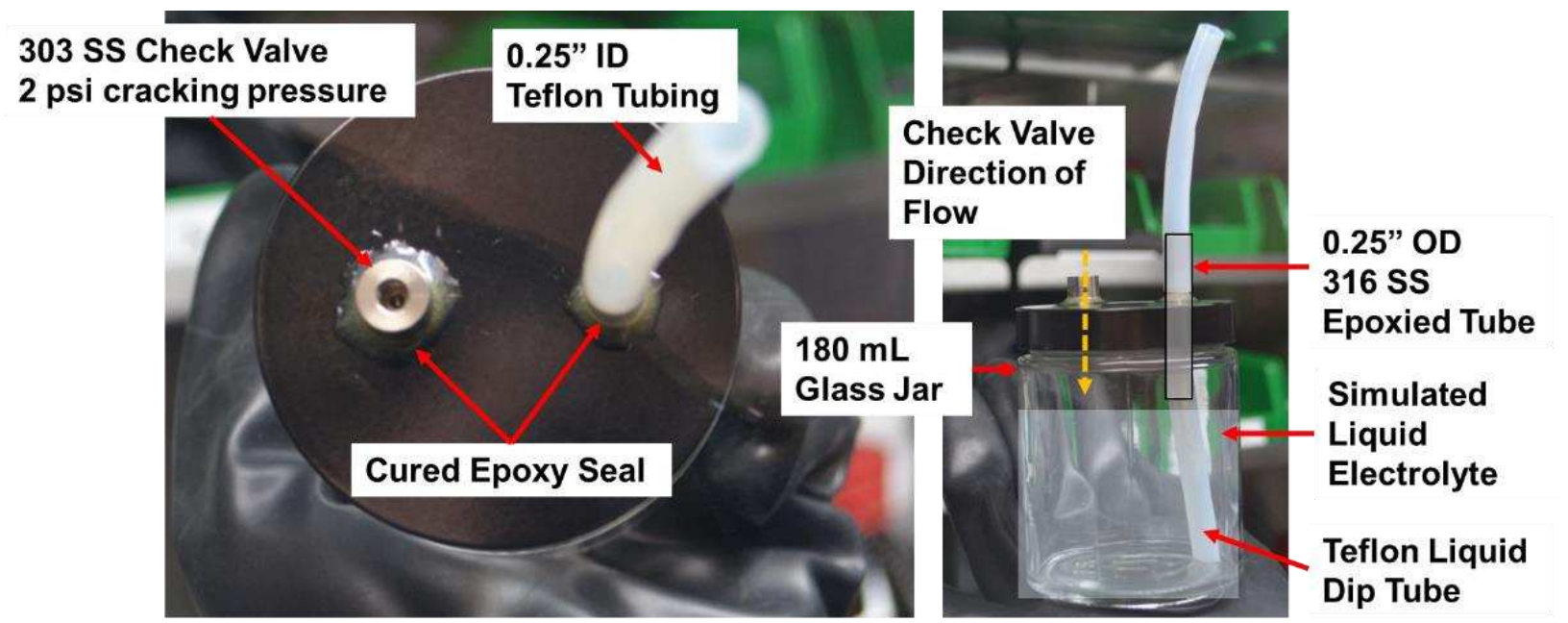

Figure 5-22: Modified $180 \mathrm{~mL}$ Glass Jar Lid for Charging the MFE into the Electrolyte Boiling Facility 
To begin the charging process, the teflon dip tube was connected to the charging valve as shown in Figure 5-23. The EBF was connected to the Trivac vacuum pump via the evacuation valve (seen in Figure 5-23). The vacuum pump was turned on and the evacuation valve was opened, while the charging valve remained closed. The EBF system pressure was monitored on the data acquisition laptop by reading the vapor pressure transmitter (described in Section 5.3.1). After the EBF was completely evacuated, the evacuation valve was closed. The charging valve was opened slightly and the liquid was observed to travel up the dip tube towards the valve. Once the liquid was drawn to the valve fitting, the charging valve was closed. The gas trapped in the dip tube line that was displaced by the liquid was evacuated from the EBF by opening the evacuation valve. After the system pressure reduced to its fully-evacuated state ( $\sim \mathrm{kPa}$ as measured by the pressure transmitter), the evacuation valve was closed. The charging valve was then slowly opened, and the water was drawn into the EBF. This process is illustrated in Figure 5-24 which plots the system pressure versus time during the valve events. While the charging valve was open and water was filling the system, the water level in the jar was carefully monitored to ensure the

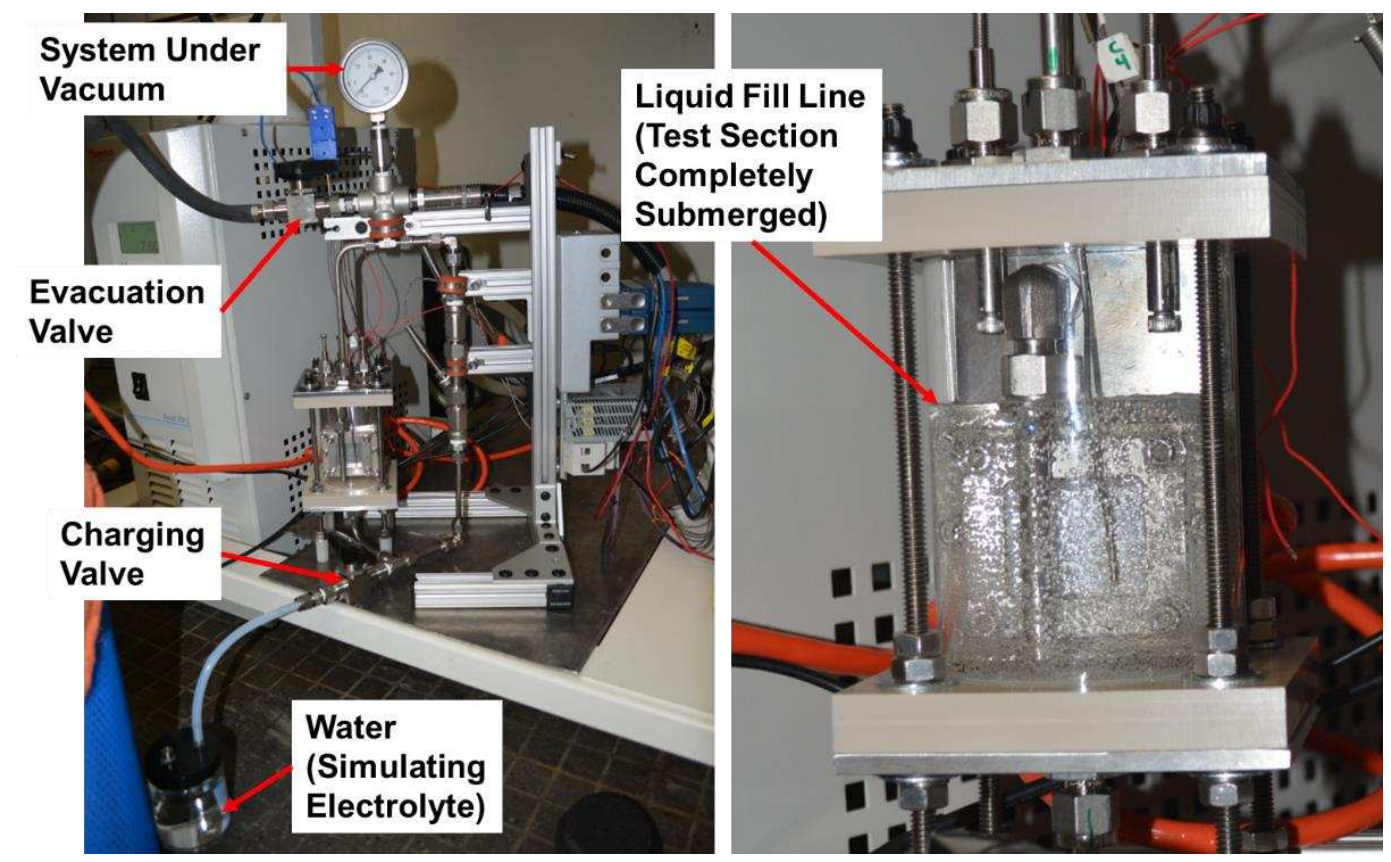

Figure 5-23: EBF Charging Process Development with Water 
liquid level never reached below the dip tube. After the test section was completely submerged, the charging valve was immediately closed and the charging process was complete. The approximate volumetric fill of the EBF to completely submerge the test section was $75 \mathrm{~mL}$.

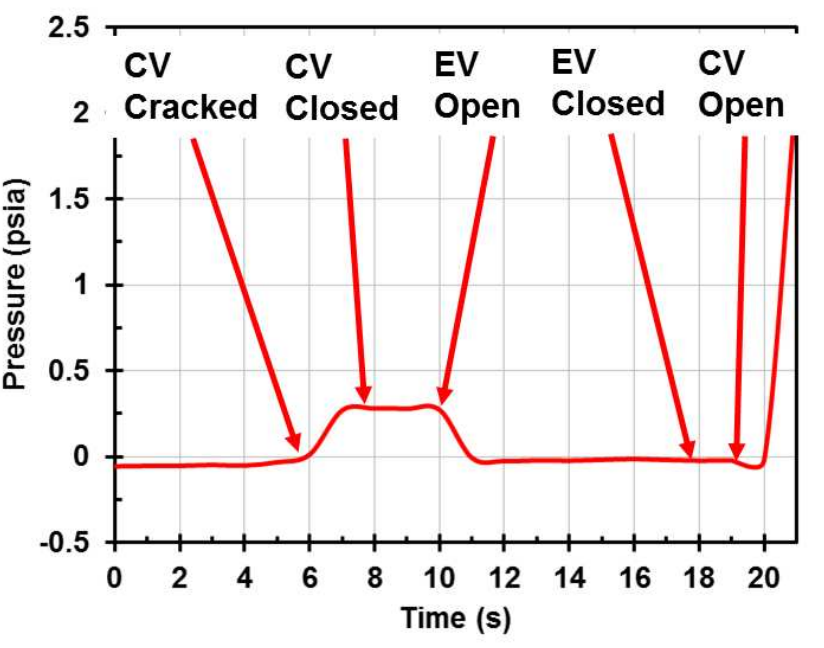

With the charging process defined, Figure 5-24: EBF System Pressure versus Time with Valve Events the MFE electrolyte for the EBF was prepared in the argon glove box. The HFE-7000 and EMC solvents were degassed using the freeze-pump-thaw procedure prior to mixing (Appendix A). The LiTFSI salt was used as received. A 1.0 M concentration of LiTFSI salt was solvated into a mixture containing 1:1 HFE-7000/EMC by volume. Approximately $100 \mathrm{~mL}$ of electrolyte was mixed in the $180 \mathrm{~mL}$ glass jar. The $25 \mathrm{~mL}$ excess of the MFE was critical to ensuring the dip tube remained completely submerged in liquid so that no argon gas was drawn into the facility. Figure 5-25 shows the $180 \mathrm{~mL}$ glass jar with modified cap containing the MFE connected to the EBF. The liquid line

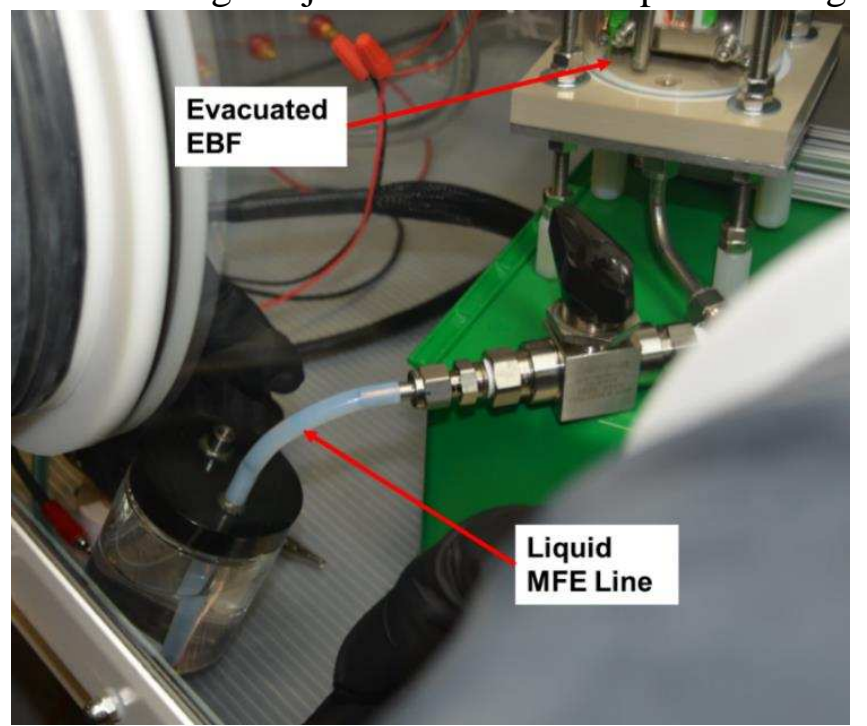
can be seen in the Teflon tube.

Figure 5-26 shows the system pressure versus time with snapshots from a recorded video of charging the EBF with the MFE. The highly volatilized mixture had a milky-white foam texture that gathered at the top of the liquid line. The texture was quickly Figure 5-25: Modified Glass Jar Containing dissipated within seconds after the charging MFE Connected to EBF for Charging 
valve was closed. It is believed that in this extreme case of boiling, the LiTFSI was precipitated from the solution to produce the milky-while color and was quickly re-solvated by the HFE-7000 and EMC solvents.

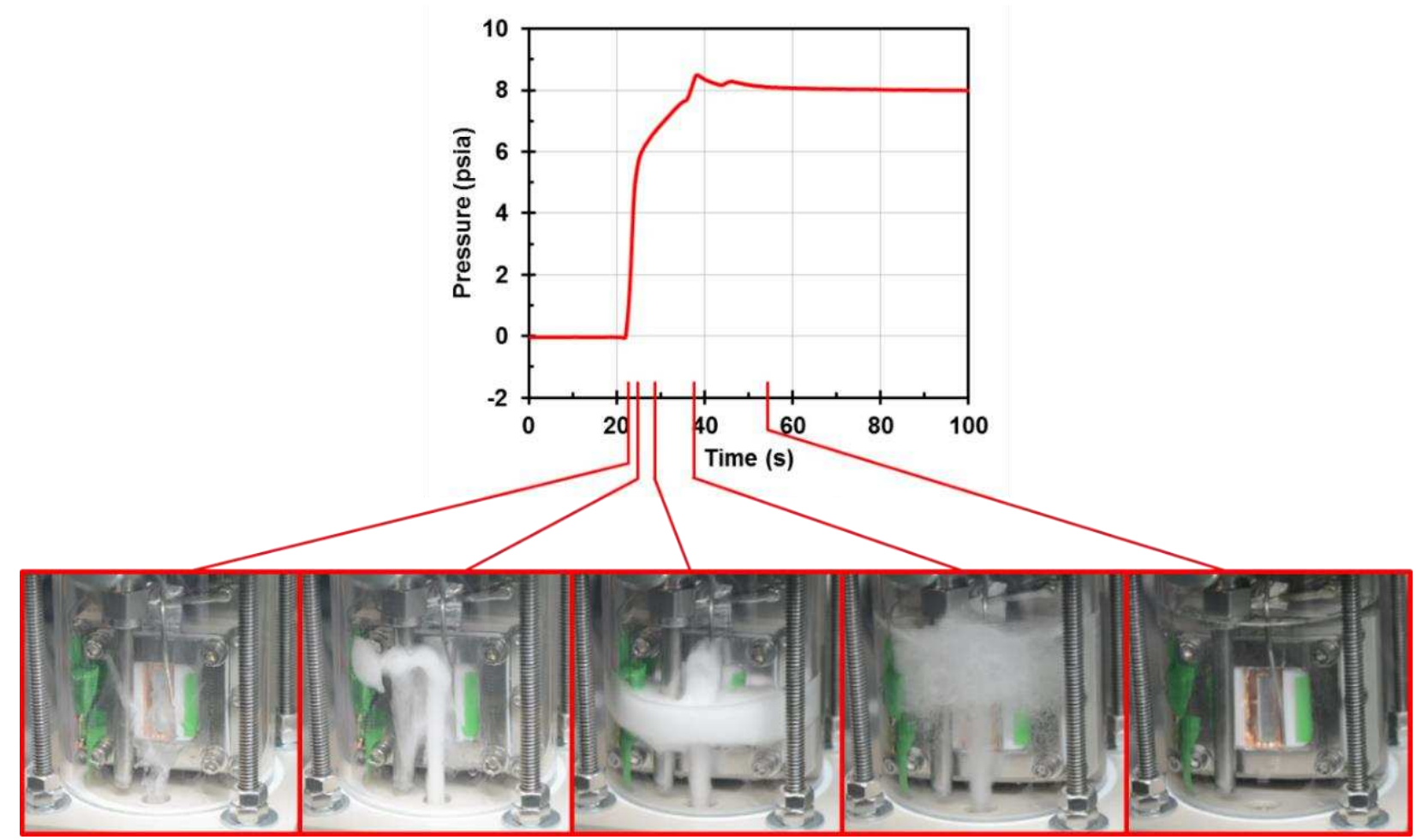

Figure 5-26: Video Snapshots of MFE Charging Process into the EBF

\subsection{Test Parameters and Equipment Used}

In the following subsections, the data acquisition system components and wiring are presented. The auxiliary components of the EBF are also presented including the recirculating chiller and DC power supplies. Thereafter, the thermal control of the EBF using the $10 \mathrm{~W}$ thin film heater and associated DC power supply is described. Finally, the galvanostatic cycling testing process and parameters are detailed. A complete list of components and equipment used for the EBF can be found in Appendix D. 


\subsubsection{Data Acquisition and Auxiliary Components}

The EBF utilizes T-type thermocouples to make several temperature measurements, all of which were 7-point water bath calibrated from $0-90^{\circ} \mathrm{C}$ using an internal platinum resistance thermometer standard with a NIST-traceable calibrated uncertainty of $\pm 0.012^{\circ} \mathrm{C}$. Further details
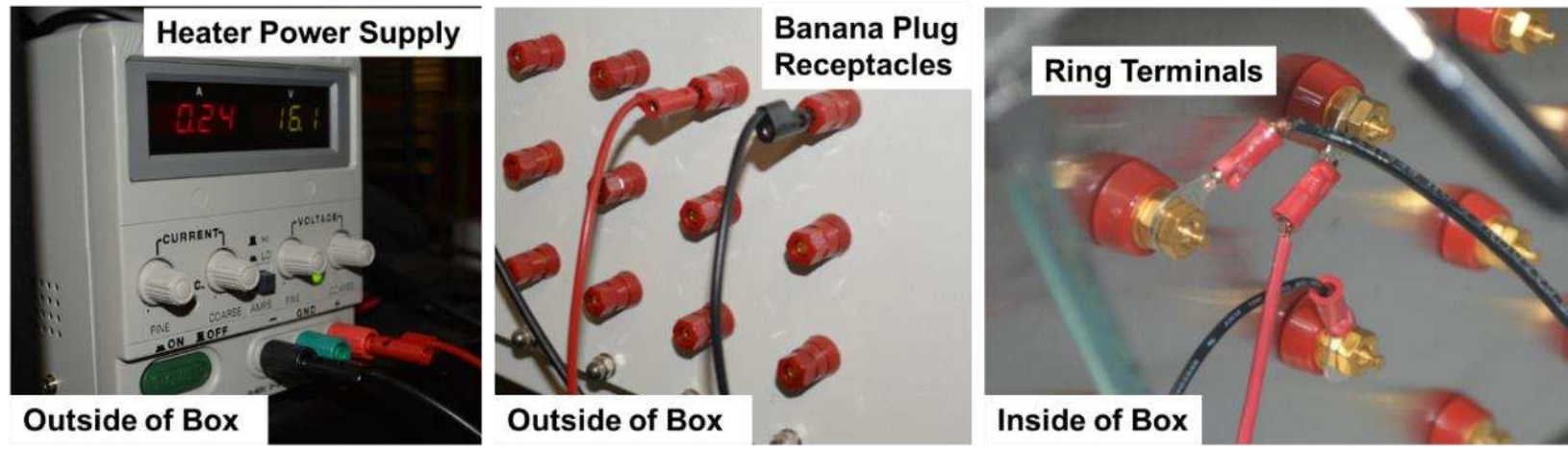

Figure 5-27: Instek SPS-606 Variable DC Power Supply for 10 W Thin Film Heater

of thermocouple calibration are provided in Appendix E. Three surface thermocouples are placed on the backside of the $10 \mathrm{~W}$ thin film heater, and the reported data for the heater temperature is an average of these three measurements (Figure 5-6).

The electrolyte bulk temperature is measured just outside of the vapor generation channel using a $1.59 \mathrm{~mm} 316$ stainless steel probe thermocouple (Figure 5-17). The water condenser inlet and outlet temperatures are measured with $6.35 \mathrm{~mm}$ 316 stainless steel NPT embedded thermocouples.

In addition, the vapor pressure of the MFE is measured with a $0-50$ psia pressure transmitter with an accuracy of $\pm 0.25 \%$ of the measurement. Power for the pressure transmitter is supplied by a

$26 \mathrm{~V}, 1.3$ A maximum DC power supply (Sola

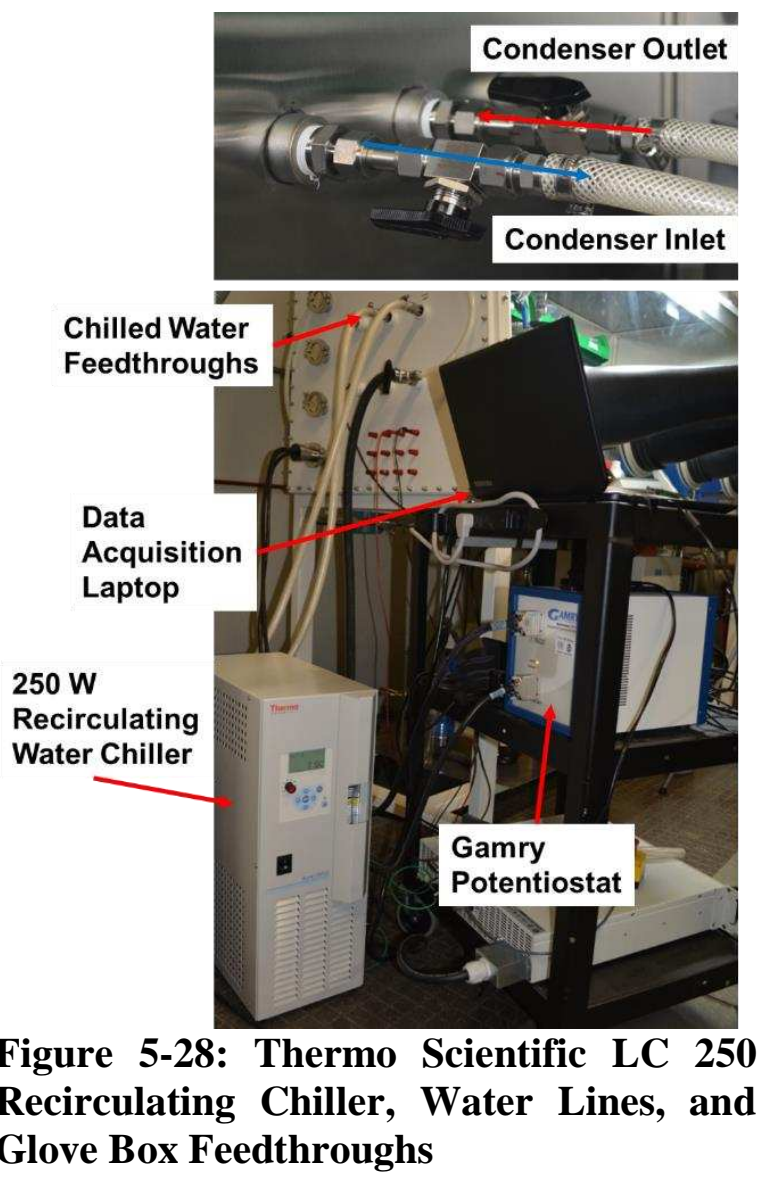




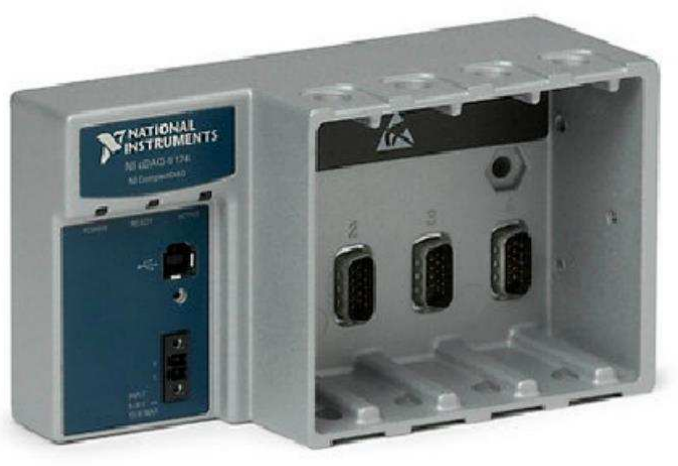

Figure 5-29: National Instruments cDAQ-9174 DAQ Chassis (source: electrical feedthrough to interface with the $10 \mathrm{~W}$ thin ni.com)
SDP1-24-100T, Figure 5-21). This power supply can also be wired to provide power to the $10 \mathrm{~W}$ preheater, if necessary. A 0-60 V, 6 A maximum variable DC power supply (Instek SPS-606) provides power to the thin film heater. Figure 5-27 shows the Instek power supply and glove box film heater contained within the EBF. Banana plug terminated wires connect to the glove box feedthrough on the outside; ring terminals provide the connection on the inside. A $250 \mathrm{~W}$ recirculating water chiller (Thermo Scientific LC 250) is connected to the shell-and-tube condenser using $12.7 \mathrm{~mm}$ ID water lines. Figure 5-28 shows the chiller, water lines, and glove box feedthroughs to interface with the condenser.

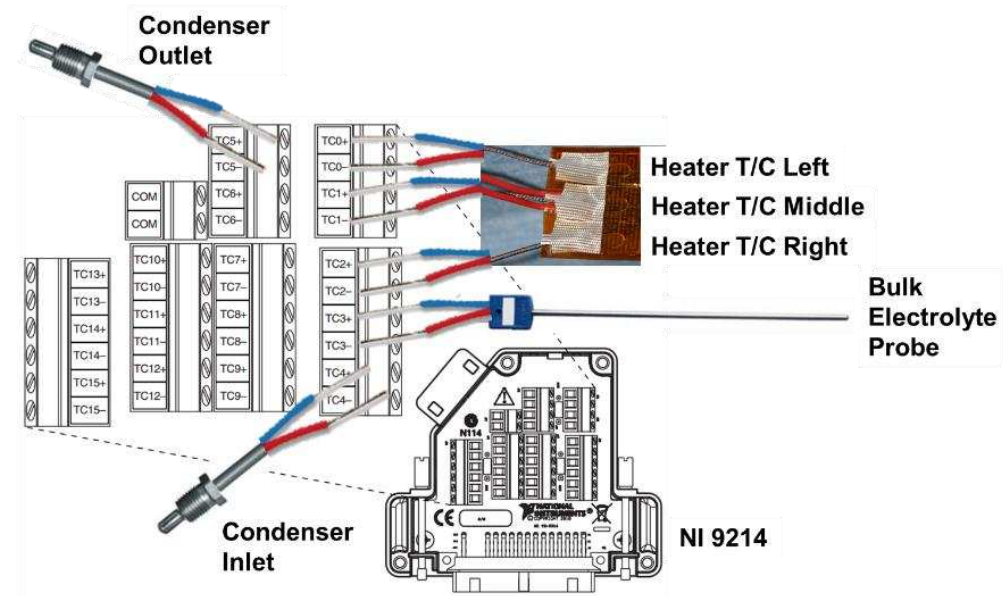

Figure 5-30: Wiring of NI 9214 16-Channel Isothermal Thermocouple Input Module

The thermocouple, pressure transmitter, and cell voltage measurements are collected using National Instruments DAQ hardware. A cDAQ-9174 chassis (Figure 5-29) is used to collect measurement signals from a NI 9214 thermocouple sensing module contained in slot 1 and a NI 9207 analog voltage and current sensing module contained in slot 2. The wiring of the NI 9214 module is shown in Figure 5-30. The heater surface thermocouples are connected in TC0-TC2. The bulk electrolyte thermocouple probe is connected to TC3. The NPT condenser thermocouples are connected to TC4-TC5. 


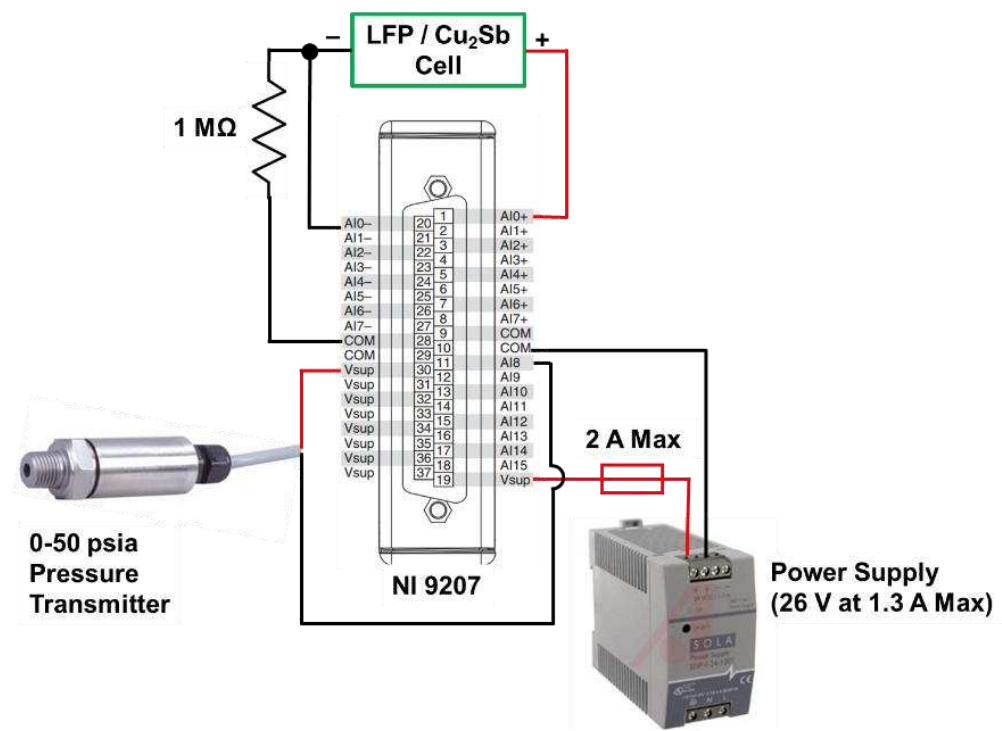

Figure 5-31: Wiring of NI 9207 Voltage/Current Analog Input Module
The wiring of the NI 9207

module is shown in Figure 5-31.

The positive lead of Sola power supply is wired directly to a $V_{\text {sup }}$ pin, 19, with a 2 A quick burn inline fuse. The ground of the power supply is attached to a common ground pin, 10. The positive pressure transmitter lead is connected to a $V_{\text {sup }}$ pin, 30, and the negative lead is connected to a current sensing channel, AI8. The voltage of the $\mathrm{LFP} / \mathrm{Cu}_{2} \mathrm{Sb}$ cell is measured via the $\mathrm{AIO}+$ and $\mathrm{AIO}$ - pins. A $1 \mathrm{M} \Omega$ resistor is wired to have a common node with the negative voltage lead and is connected to the common ground (pin 28). The addition of the $1 \mathrm{M} \Omega$ resistor removed the noise associated with measuring the floating differential voltage signal of the cell relative to the DAQ module.

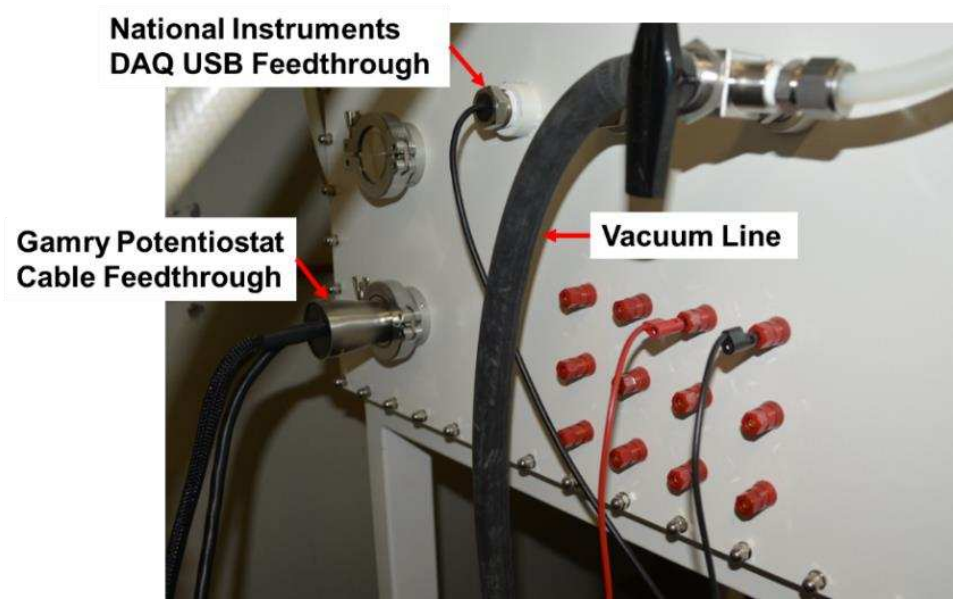

Figure 5-32: Glove Box Feedthroughs for NI DAQ and Gamry Potentiostat
The cDAQ-9174 chassis was attached to the side of the $80 / 20$ aluminum support frame inside the glove box (Figure 5-21). The chassis communicated with the data acquisition laptop via a USB cable hermetically sealed in a glove box feedthrough (Figure 5-32). 
A Virtual Instrument (VI) was created in LabVIEW software to collect, calibrate, display, and record the data from the sensors contained within the EBF. The VI Front Panel is seen on the data acquisition laptop screen in Figure 5-33. In separate charts, the bulk electrolyte temperature, vapor pressure, heater temperatures, and chiller water temperatures were displayed. The VI output a *.TDMS binary file type that contains the recorded measurements. A Microsoft Excel plug-in was used to import the National Instruments binary data file into an easily manipulated spreadsheet format.

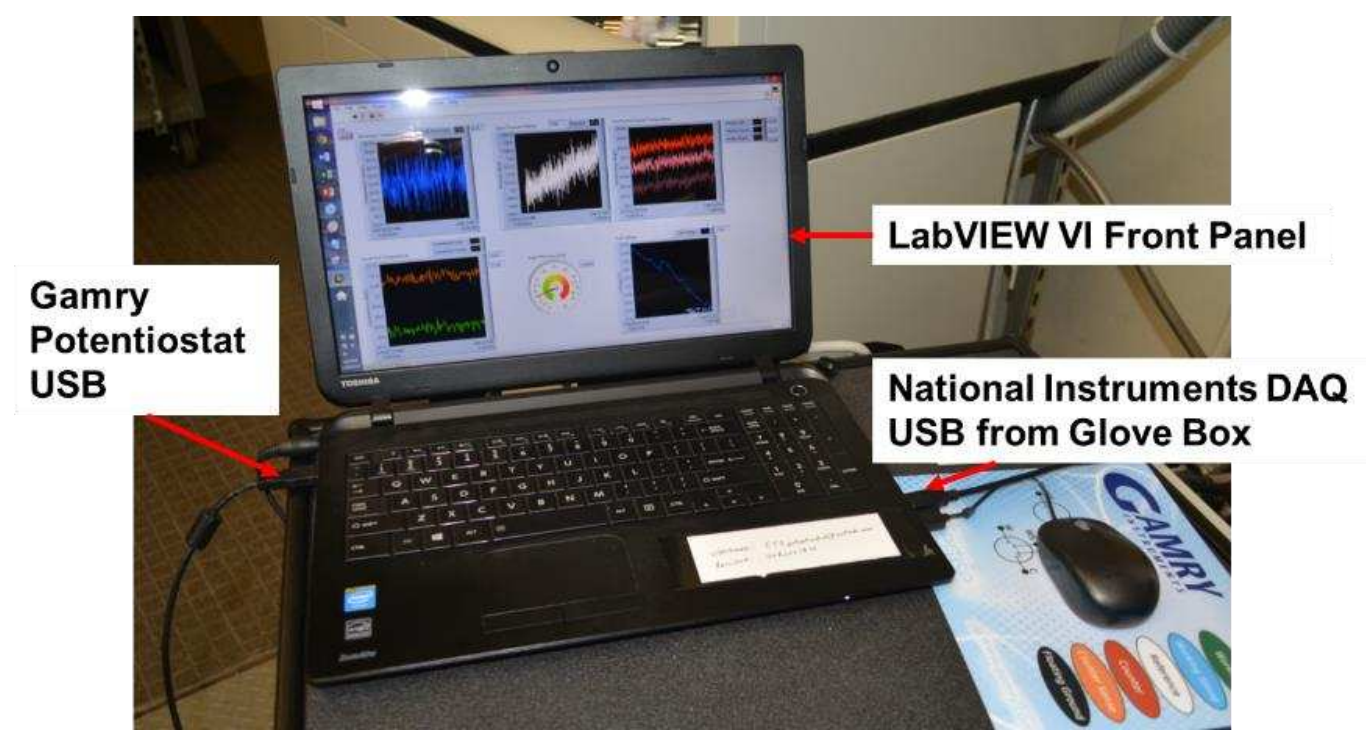

Figure 5-33: VI Front Panel for EBF DAQ System

\subsubsection{Thermal Control of Electrolyte Boiling Facility}

The amount of heat input required to induce nucleate boiling in the LFP positive electrode channel was determined iteratively. After the EBF was charged with the MFE, the variable DC power supply that controlled the heater (Figure 5-27) was turned set to $1 \mathrm{~W}$, and the evaporation channel was monitored for vapor bubble generation. After 10 minutes, if the continuous vapor bubble generation was not observed, the power supply output was increased by $0.5 \mathrm{~W}$. During the experiments, $4 \mathrm{~W}$ produced the desired continuous vapor generation in the channel and was used as the set point for the boiling and galvanostatic cycling experiments. Due to the sufficiency of the 
$4 \mathrm{~W}$ supplied by the thin film heater on the backside of the LFP electrode, the preheater was not used for any of the boiling experiments.

In initial testing with the EBF, a significant system thermal time constant was observed when the heater was turned on or off. This is mainly due to the large thermal mass of the stainless steel filler, which required a significant amount of time to reach thermal equilibrium with the MFE and the surroundings. Approximately 7 hours were required for the system temperature and pressure to stabilize. The galvanostatic cycling experiments were only performed once equilibrated thermal conditions existed for both boiling and non-boiling experiments.

\subsubsection{Galvanostatic Cycling}

A Gamry Reference 3000 Potentiostat (Figure 4-8) was used to perform the galvanostatic cycling of the LFP/Cu $\mathrm{Cu}_{2} \mathrm{Sb}$ cell in the EBF. A set of $3 \mathrm{~m}$ potentiostat cables were hermetically sealed into the glove box feedthrough (seen in Figure 5-32) which allowed the potentiostat to perform the cycling experiments while outside of the glove box. The same calibration procedure described in Section 4.3.2 was used.

The $\mathrm{LFP} / \mathrm{Cu}_{2} \mathrm{Sb}$ cell cycling voltage limits were $1.75-3.5 \mathrm{~V}$, the same used in full cell testing described in Section 4.5.2. The testing procedure was split into three segments: (1) preboiling cycling, (2) boiling and cycling, and (3) post-boiling cycling (Table 5-2). The pre-boiling cycling was accomplished in cycles $1-10$. The cell was initially charged at $51.7 \mu \mathrm{A} \mathrm{cm}^{-2}(0.15$ $\mathrm{mA})$. All remaining cycles were performed with a cycling current of $172 \mu \mathrm{A} \mathrm{cm}^{-2}(0.5 \mathrm{~mA})$ which is approximately a $0.5 \mathrm{C}$ rate. Cycles $1-10$ were used to establish an electrochemical performance baseline prior to boiling the MFE. After the completion of cycle 10, the DC power supply was turned on to apply $4 \mathrm{~W}$ to the thin film heater, the re-circulating water chiller was turned on and set to $8^{\circ} \mathrm{C}$, and the cell was placed on a 7 hour rest for thermal steady-state conditions to be 
achieved. After the rest, the cell was cycled 10 times while the MFE was continuously boiled (cycles 11-20). Upon the completion of cycle 20, the power supply for the thin film heater was turned off and the cell is placed on a 7 hour rest to achieve non-boiling thermal equilibrium. Once cooled back to room temperature, the cell was cycled 10 additional times (cycles 21-30) to assess the electrochemical impact of the boiling electrolyte during cycles 11-20.

Table 5-2: Testing Procedure for Electrolyte Boiling Facility

Test EBF Testing

Segment Event Test Parameters Notes

\begin{tabular}{|c|c|c|c|}
\hline (1) & First Charge & $\begin{array}{l}51.7 \mu \mathrm{A} \mathrm{cm}^{-2} \text { until } 3.5 \mathrm{~V} \\
\text { at room temperature }\end{array}$ & Only applied for first charge \\
\hline (1) & Cycles 1-10 & $\begin{array}{l}172 \mu \mathrm{A} \mathrm{cm}^{-2} \text { from } 1.75- \\
3.5 \mathrm{~V} \text { at room temperature }\end{array}$ & $\begin{array}{l}\text { Establish electrochemical } \\
\text { performance baseline pre-boil }\end{array}$ \\
\hline (2) & Heater On & $4 \mathrm{~W}$, chiller set to $8^{\circ} \mathrm{C}$ & Wait 7 hours for thermal equilibrium \\
\hline (2) & Cycles $11-20$ & $\begin{array}{l}172 \mu \mathrm{A} \mathrm{cm}^{-2} \text { from } 1.75- \\
3.5 \mathrm{~V} \text { with } 4 \mathrm{~W} \text { heat input }\end{array}$ & $\begin{array}{l}\text { Measure electrochemical performance } \\
\text { with continuous co-solvent } \\
\text { evaporation }\end{array}$ \\
\hline (2) & Heater Off & Chiller off & Wait 7 hours for thermal equilibrium \\
\hline (3) & Cycles 21-30 & $\begin{array}{l}172 \mu \mathrm{A} \mathrm{cm}^{-2} \text { from } 1.75- \\
3.5 \mathrm{~V} \text { at room temperature }\end{array}$ & Assess impact of boiling electrolyte \\
\hline
\end{tabular}

\subsection{Results and Discussion}

The pre-boiling data of cycles 1-10

is seen in Figure 5-34 and Figure 5-35. The first charge capacity was approximately $1.06 \mathrm{mAh} \mathrm{cm}^{-2}$, with a first cycle coulombic efficiency (CE) of $37.4 \%$ (Figure 5-35). The $\mathrm{CE}$ of the $\mathrm{LFP} / \mathrm{Cu}_{2} \mathrm{Sb}$ cell in the boiling facility was much lower than that observed in Section 4.5 with the same electrode architecture in PFA T-cells

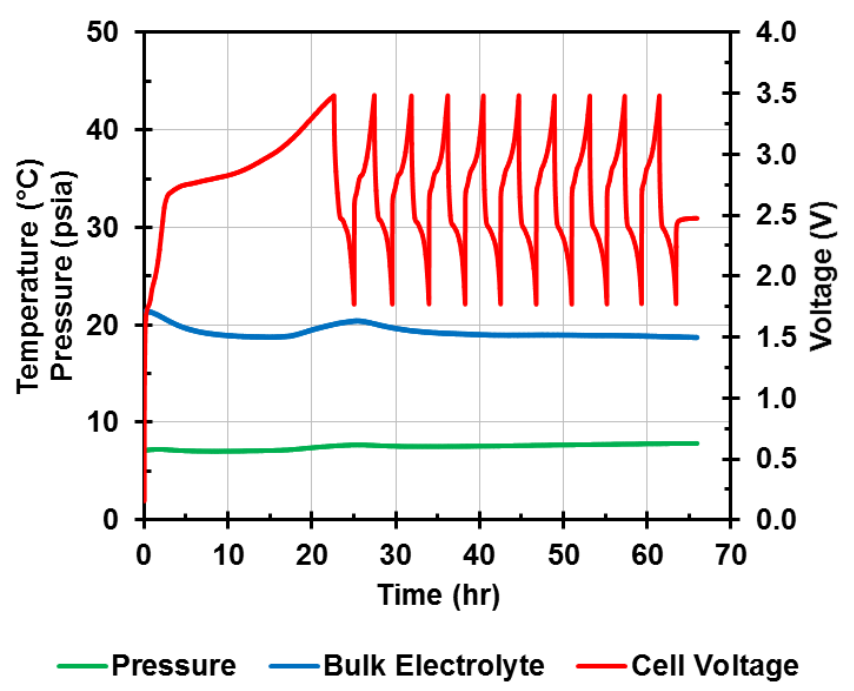

Figure 5-34: Pre-Boiling Chronopotentiogram of $\mathrm{LFP} / \mathrm{Cu}_{2} \mathrm{Sb}$ Cell during Cycles 1-10 with System Temperature and Pressure Traces 


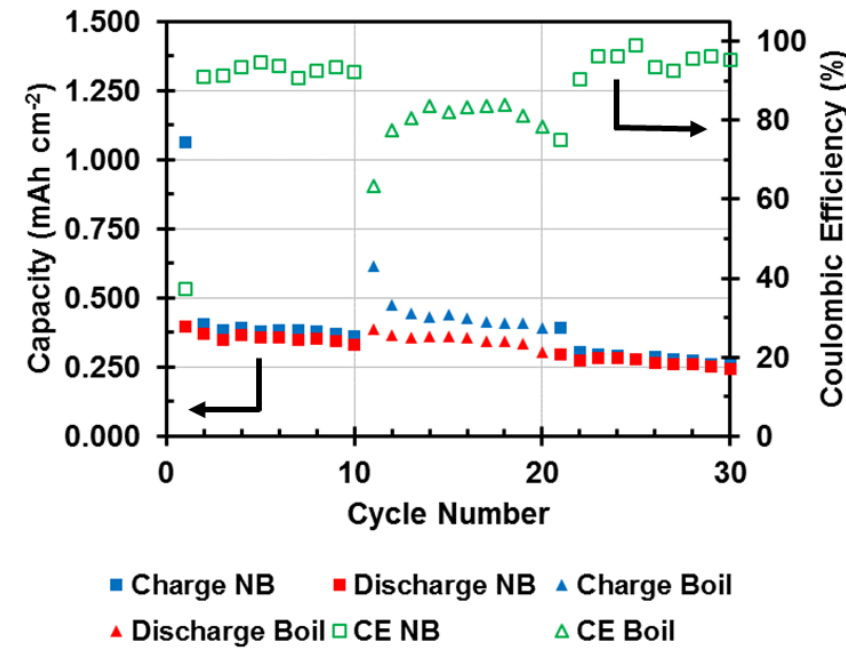

Figure 5-35: LFP/Cu2Sb Cell Capacity and dropped to approximately $0.347 \mathrm{mAh} \mathrm{cm}^{-2}$ Coulombic Efficiency versus Cycle Number

$(83 \%)$. The large first charge capacity loss is attributed to the formation of the SEI on both the anode and cathode interfaces as well as reactions with any surface oxides on the active materials and wetted components of the boiling facility. After cycle 10 , the reversible cycling capacity $\left(76.3 \mathrm{mAh} \mathrm{g}^{-1}\right)$. This capacity is lower than the reversible capacity of the LFP/Cu $2 \mathrm{Sb}$ full cell tests conducted in Chapter $4\left(\sim 150 \mathrm{mAh} \mathrm{g}^{-1}\right)$. The lower capacity is most likely due to the additional decomposition products formed on the electrode surfaces from excess manipulation outside of the glove box for EBF assembly. During these cycles, the bulk electrolyte temperature and absolute pressure of the MFE were, on average, $19.3^{\circ} \mathrm{C}$ and $51.7 \mathrm{kPa}(7.5 \mathrm{psia})$, respectively. As expected, HFE-7000 appears to be the primary contribution to the high vapor pressure of the MFE. HFE-7000 in pure form has a vapor pressure of $55.6 \mathrm{kPa}$ at $19.3^{\circ} \mathrm{C}$. The slight temperature and pressure fluctuations seen over the course of the 65 hours of this portion of the experiment are due to changes in the room temperature, which were present in all phases of the experiment. The cell appears to have normal operation throughout the 10 cycles and establishes an electrochemical performance baseline prior to boiling.

At the conclusion of cycle 10, $4 \mathrm{~W}$ were supplied to the thin film heater. Vapor generation was immediately observed ( $<1.5$ seconds) in the evaporation channel. The intensity of the vapor generation increased as both the heater and electrolyte temperatures increased. After approximately 7 hours, the heater and bulk electrolyte temperatures reach thermal equilibrium, and 
cycles 11-20 were started (Figure 5-36).

Interestingly, while the heater and bulk

electrolyte temperatures increased, the

voltage of the cell decreased under open-

circuit conditions. Cell thermodynamics

predict the potential to increase with

increasing temperature. This indicates

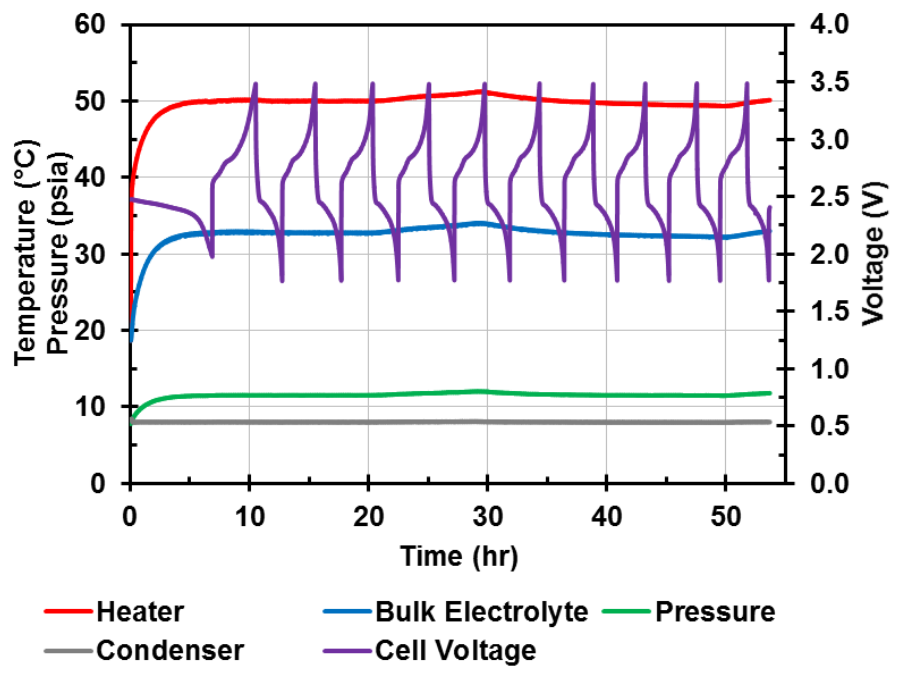

that the cell in the current experiment is

self-discharging as the system warms to

Figure 5-36: Boiling Chronopotentiogram of LFP/Cu2Sb Cell during Cycles 11-20 with System Temperature and Pressure Traces

thermal equilibrium. Over the course of the 7 hours, the cell voltage dropped by approximately 0.5

V. Bandhauer et al. states reversible self-discharge is caused by the dissolution of surface species, such as the SEI. The dissolution increases the reactivity of the active materials which, in turn, increases the rate of self-discharge [3]. It appears this is the case for the LFP/Cu $2 \mathrm{Sb}$ cell, which has already demonstrated reduced performance in stable SEI formation with the MFE in prior nonboiling experiments.

The average heater surface and bulk electrolyte temperatures during cycles 11-20 were $50.0^{\circ} \mathrm{C}$ and $32.9^{\circ} \mathrm{C}$, respectively. The average vapor pressure during the same time was $80.19 \mathrm{kPa}$ (11.63 psia). The water circulating through the condenser remained at a constant temperature of $8^{\circ} \mathrm{C}$ throughout cycles $11-20$. The boiling temperature of the MFE, $32.9^{\circ} \mathrm{C}$, is critical in evaluating the feasibility of the proposed internal TMS. These results show that the MFE can be continuously boiled at temperatures lower than those associated with capacity fade and thermal runaway. The isothermal heat absorption provided by boiling HFE-7000 allows the cell to maintain safe 
operation temperatures while effectively dissipating a very large heat flux $\left(1.4 \mathrm{~W} \mathrm{~cm}^{-2}\right)$ applied to the positive electrode.

To better understand the thermal dissipation performance of the $\mathrm{LFP} / \mathrm{Cu}_{2} \mathrm{Sb}$ cell with the MFE, the applied heat flux is compared to heat flux of the cells in the Chevy Volt battery pack. Hamut et al. reports the Chevy Volt battery TMS is designed to manage $0.35 \mathrm{~kW}$ of heat generation from the 288-15 Ah pouch cells of the pack [64], each of which contains 16 unit cells (two-sided cathode, separator, two-sided anode) with approximate electrode dimensions of $19.2 \times 14.5 \mathrm{~cm}$ [30]. By estimating the electrode area, and normalizing the reported heat generation, the approximate heat flux experienced by the electrodes of the cells during normal operation is 136 $\mu \mathrm{W} \mathrm{cm}{ }^{-2}$, or four orders of magnitude lower than the heat flux experienced by the $\mathrm{LFP} / \mathrm{Cu}_{2} \mathrm{Sb}$ cell during this experiment. Therefore, the results shown in Figure 5-36 represent an extreme case of heat flux, such as thermal runaway of a neighboring cell in a large battery pack. Not only did the cell manage to dissipate the heat while maintaining a safe operating temperature, it was also capable of cycling.

The chronopotentiogram in Figure 5-36 and the charge-discharge capacity in Figure 5-35 show the effect of the self-discharge during the 7 hour rest prior to cycle 11 . The $11^{\text {th }}$ cycle charge had a capacity of $0.616 \mathrm{mAh} \mathrm{cm}^{-2}$. This is nearly double the charge capacity during the $10^{\text {th }}$ cycle charge, $0.361 \mathrm{mAh} \mathrm{cm}^{-2}$, indicating that additional electrolyte decomposition products are formed to re-passivate the active material surface. It is also possible that the cell was slowly discharged by the test fixture during the 7 hour rest due to a small electrical short, but this is unlikely because physical separation of the anode and cathode created by the $50 \mu \mathrm{m}$ Teflon sheet in the electrode stacking structure. After the $11^{\text {th }}$ cycle, the cell cycles more reversibly and the coulombic efficiency improves. However, the cell never achieves a coulombic efficiency greater than $84 \%$ 
during cycles 11-20. The reduced coulombic efficiency during the boiling experiments can be attributed to two different mechanisms: $\mathrm{Li}_{3} \mathrm{Sb}$ formation in the $\mathrm{Cu}_{2} \mathrm{Sb}$ anode and continual SEI decomposition. $\mathrm{Li}_{3} \mathrm{Sb}$ has more favorable formation kinetics at higher temperatures. $\mathrm{Cu}_{2} \mathrm{Sb}$ is known to have high sensitivity to the potential vs. $\mathrm{Li} / \mathrm{Li}^{+}$, and slight capacity fade is expected when cycled to potentials lower than $0.5 \mathrm{~V}$ vs. $\mathrm{Li} / \mathrm{Li}^{+}[128]$. In the $\mathrm{LFP} / \mathrm{Cu}_{2} \mathrm{Sb}$ cell of this experiment, the $\mathrm{Cu}_{2} \mathrm{Sb}$ anode had the limiting capacity of the two electrodes. Therefore, its potential vs. $\mathrm{Li} / \mathrm{Li}^{+}$ varied much more significantly and was more difficult to control. Potentials lower than $0.5 \mathrm{~V}$ vs. $\mathrm{Li} / \mathrm{Li}^{+}$causes excess formation of $\mathrm{Li}_{3} \mathrm{Sb}$ and loss of electrical contact with the surrounding copper, preventing de-intercalation of lithium. This causes a high charge capacity and a low discharge capacity. The higher cell temperatures did induce slight SEI decomposition, most notably during the self-discharge during the rest period before cycle 11. Because the higher cell temperature is maintained during the boiling, this slight SEI decomposition is expected to continue during the cycling of the cell, contributing to the lower coulombic efficiency. Evaporating HFE-7000 from the MFE appears to have a smaller effect on the capacity fade and reduced coulombic efficiency than $\mathrm{Li}_{3} \mathrm{Sb}$ formation in the anode and SEI decomposition due to higher cell temperatures. In future experiments, revised cell voltage limits are required to better manage the capacity-limiting $\mathrm{Cu}_{2} \mathrm{Sb}$ anode. In addition, investigations into the inclusion of SEI stabilizing additives in the MFE, such as $\mathrm{EC}$ and $\mathrm{LiPF}_{6}$, could help to reduce the amount of SEI decomposition when the temperature of the cell is increased. Arai showed significant electrochemical improvement by utilizing these additives in electrolytes that contained HFE-7100, a very similar fluid to HFE-7000 [86]. 


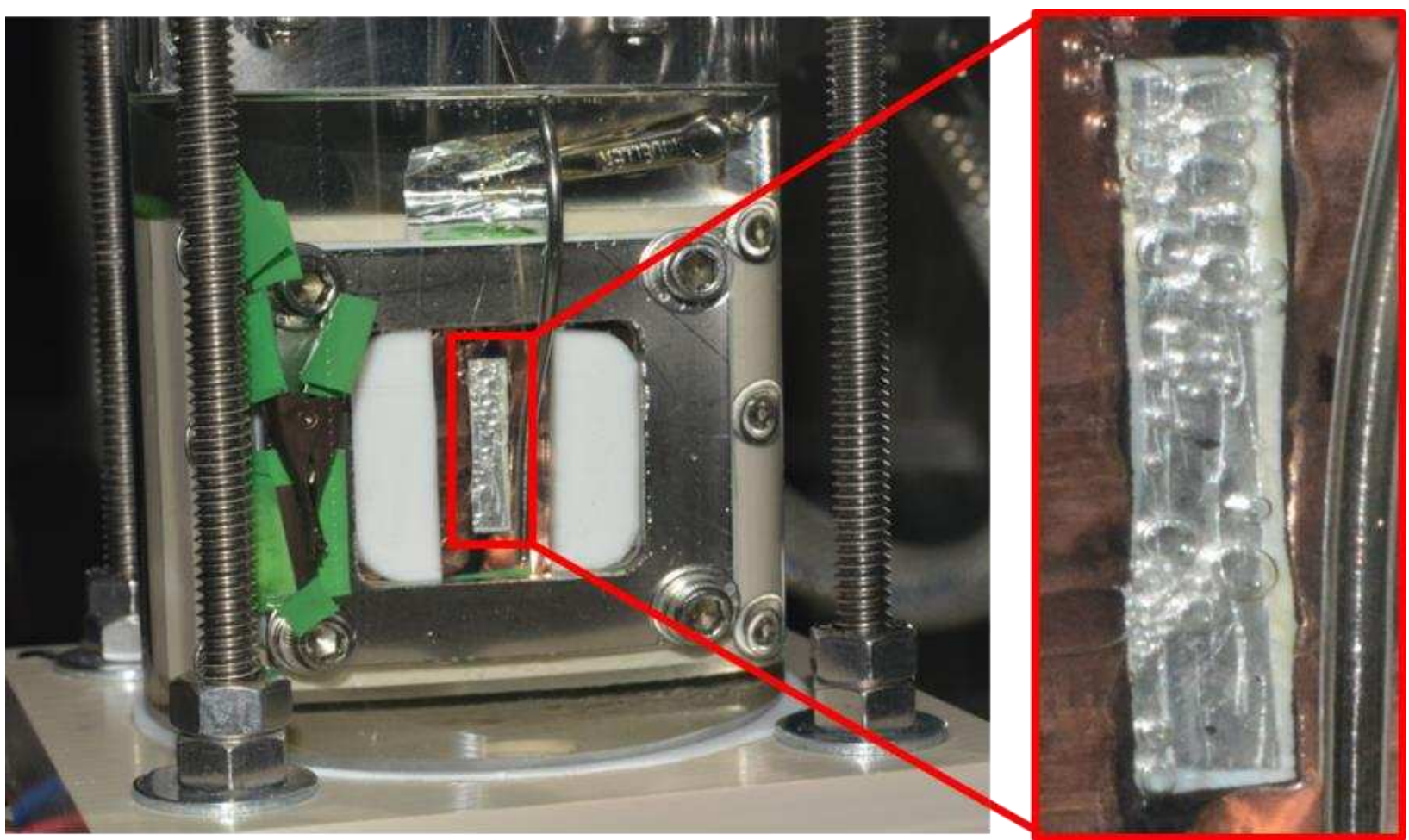

Figure 5-37: View of Evaporation Channel while Cycling LFP/Cu2Sb Cell at 0.5C with 4 W of Heat Input

Figure 5-37 shows a representative snapshot of the continuous vapor generation in the evaporation channel of the LFP electrode during cycles 11-20. The elongation of the bubbles indicates the boiling was confined into the channel bounded by the $1 \mathrm{~mm}$ thick piece of glass on the backside of the $\mathrm{Cu}_{2} \mathrm{Sb}$ electrode (see Figure 5-10 and Figure 5-18). Upon sufficient heat absorption, the vapor bubbles rapidly rose to exit the top of the channel and were just as rapidly replaced by the formation of additional vapor bubbles. The high vapor pressure of the MFE indicates that HFE-7000 accounts for the majority of the vapor phase, and, therefore, it is the primary constituent boiled in the channel. Visual observation showed no lithium salt precipitation at any location within the test facility, including the evaporation channel. This result was not unexpected. Arai and Dokko et al. have measured the role of HFEs in solvation of $\mathrm{Li}^{+}$. Arai demonstrated with C-NMR solvation shift measurements that HFE-7100 participated minimally in $\mathrm{Li}^{+}$solvation of LiBETI salt when mixed with EMC [85]. Dokko et al. used pulsed-gradient 
spin-echo NMR to evaluate the self diffusion coefficients of $\mathrm{Li}^{+}$and HFE-458 in a triglyme and LiTFSI salt electrolyte, and found the HFE had increased diffusivity due to no participation in $\mathrm{Li}^{+}$ solvation in comparison to its triglyme counterpart [140, 141]. Due to direct similarities in molecular structure of HFE-7000 and HFE-7100, the same solvation performance of $\mathrm{Li}^{+}$was expected in the MFE. Therefore, the increased availability of HFE-7000 for evaporation, due to minimal participation in $\mathrm{Li}^{+}$solvation, further supports the feasibility of the proposed internal TMS.

The measured heater and bulk electrolyte temperatures during cycles 11-20 were compared to thermodynamic calculations based on ideal mixture assumptions of the HFE-7000 and EMC fluids. These calculations did not incorporate the effect of LiTFSI salt. Two component ideal mixtures can be modeled using the following system of equations [142]:

$$
\begin{gathered}
y_{\mathrm{HFE}-7000} P_{\mathrm{v}}=x_{\mathrm{HFE}-7000} P_{\mathrm{HFE}-7000}^{\text {sat }} \\
y_{\mathrm{EMC}} P_{\mathrm{v}}=x_{\mathrm{EMC}} P_{\mathrm{EMC}}^{\text {sat }} \\
y_{\mathrm{HFE}-7000}+y_{\mathrm{EMC}}=1 \\
x_{\mathrm{HFE}-7000}+x_{\mathrm{EMC}}=1
\end{gathered}
$$

where $y_{\mathrm{i}}$ is the vapor molar fraction of either EMC or HFE-7000, $P_{\mathrm{v}}$ is the total system vapor pressure, $x_{\mathrm{i}}$ is the liquid molar fraction of EMC or HFE-7000 in the MFE mixture, and $P_{\mathrm{i}}^{\text {sat }}$ is the saturation pressure of either the EMC or HFE-7000 liquid. Equation (5.1) is Raoult's Law which describes the system vapor pressure contribution of the HFE-7000 fluid based upon its liquid molar fraction in the MFE mixture. Similarly, Equation (5.2) describes the contribution to the system vapor pressure of the EMC fluid based on its molar mixing ratio. Equations (5.3) and (5.4) describe that only HFE-7000 and EMC comprise the ideal mixture in the vapor and liquid phase, respectively. The two component ideal mixture model contains six unknowns in four equations. 
To solve this system, two independent variables are required: fluid temperature and system vapor pressure. Therefore, for any predetermined temperature and pressure, the mole fractions of HFE7000 and EMC in the liquid and vapor phase can be calculated.

The thermodynamic properties of HFE-7000 are well understood and available in EES software. Unfortunately, the thermodynamic properties of EMC are not defined in commercial software. However, a study has been performed to determine the vapor pressure of EMC as a function of temperature, which is sufficient for performing this ideal mixture analysis [143]. In this study, the vapor pressure measurements of EMC were fit using the Antoine Equation which has the general expression:

$$
\log \left(P_{\mathrm{EMC}}^{\text {sat }}\right)=A-\frac{B}{T+C}
$$

where $A$ is $6.4308, B$ is 1466.437 , and $C$ is -49.461 for EMC [143].

Bubble point-dew point graphs are useful for visualizing the equilibrium vapor and liquid composition in two component fluid mixtures. The bubble point line defines the lowest temperature at which vapor will be generated in the mixture by the evaporation of the most volatile of the two liquids. The dew point line defines the temperature at which the ideal vapor mixture will begin to condense. To generate bubble point-dew point curves, the liquid molar fraction of one component is varied between 0 and 1 and a single system vapor pressure $\left(P_{\mathrm{v}}\right)$ is defined. Therefore, the systems of equations described above can be solved for the molar fractions of the

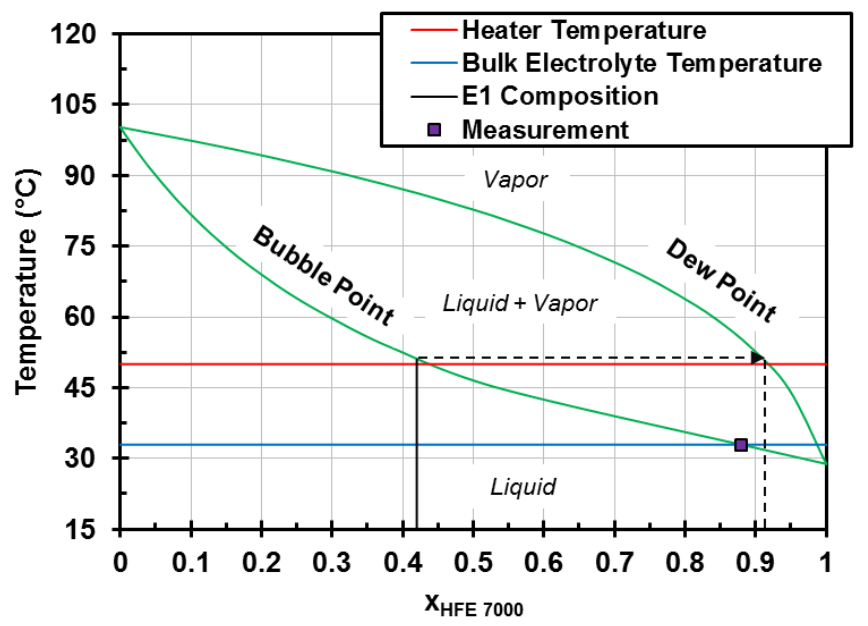

Figure 5-38: Bubble Point-Dew Point of HFE7000/EMC Mixture with Measured Heater and Bulk Electrolyte Temperature from EBF Test 
vapor phase and the saturation temperature of both HFE-7000 and EMC.

Figure 5-38 shows the bubble point-dew point curve of an ideal HFE-7000/EMC mixture. Since HFE-7000 has a much greater vapor pressure than EMC, the 1:1 HFE-7000/EMC by volume liquid mixture (i.e. $x_{\mathrm{HFE}-7000}=0.42$ ) is expected to have a vapor composition that contains approximately $92 \%$ HFE-7000 by molar ratio. The vapor composition is determined by tracing the liquid molar composition to the bubble point line. A horizontal line can then be drawn to the dew point line. The molar composition at which the horizontal line and the dew point line intersect is the vapor molar composition. Because HFE-7000 is $92 \%$ of the vapor mixture under equilibrium conditions, the vapor generation observed in the EBF is primarily HFE-7000. Figure 5-38 also contains the average temperatures of the heater and bulk electrolyte during the MFE boiling. Based upon these two physical measurements, it is expected that the actual temperature of the boiling electrolyte would be in between these two values. Interestingly, the heater temperature and the expected bubble point of the HFE-7000/EMC mixture are in close agreement. This result suggests that the effect of the LiTFSI salt might not be significant, and that the fluid evaporation temperature is very close to the heater temperature. More investigation of the effect of salt concentration on the bubble point is warranted.

Figure 5-39 shows the effect of system pressure on the bubble point-dew point curves of the HFE-7000/EMC mixture. It can clearly be seen that boiling temperature of the mixture increases with increased in system pressure. LIBs generate gas during SEI formation cycles

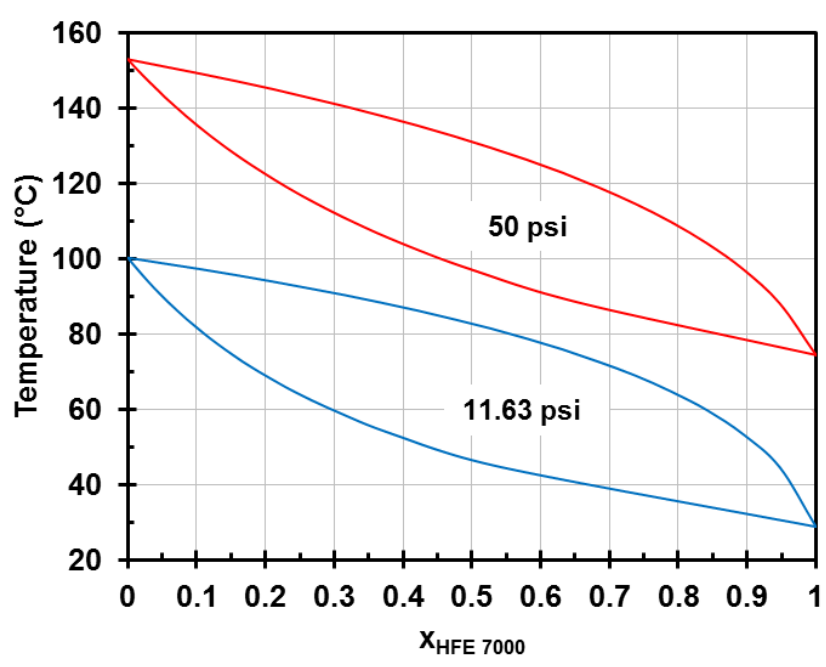

Figure 5-39: Effect of System Pressure on Bubble Point-Dew Point of HFE-7000/EMC Mixture 
which will contribute to increased cell pressure. In addition, gas generation occurs during SEI decomposition as well, which was evident by the lower coulombic efficiencies seen during the boiling cycles. The effect of gas generation, due to SEI formation and decomposition, on the boiling point of the MFE mixture warrants further investigation.

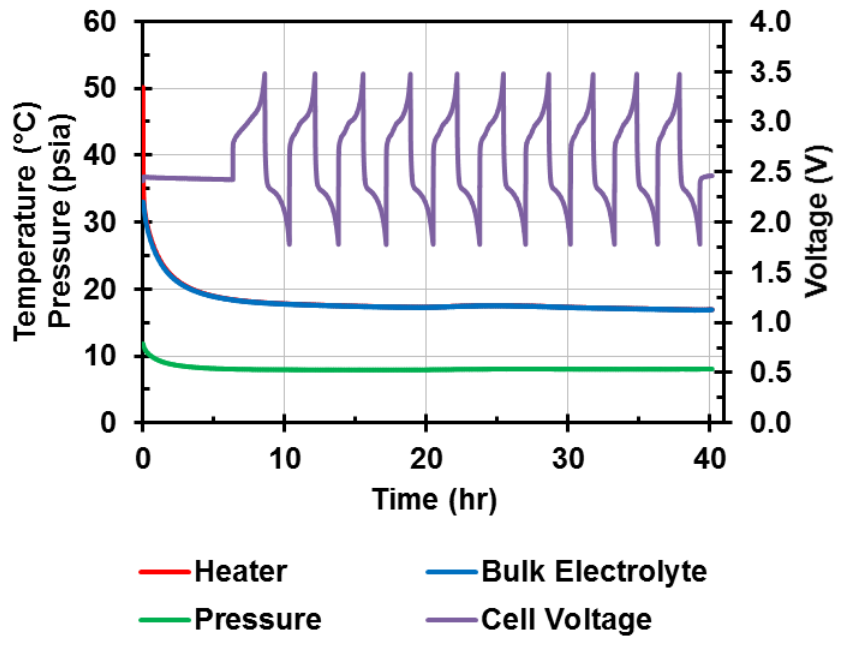

Figure 5-40: Post-Boiling Chronopotentiogram of LFP/Cu2Sb Cell during Cycles 21-30 with System Temperature and Pressure Traces

Figure 5-40 shows the post-boiling data collected on the $\mathrm{LFP} / \mathrm{Cu}_{2} \mathrm{Sb}$ cell. After the 7 hour rest, the bulk electrolyte cooled to an average temperature of $17.35^{\circ} \mathrm{C}$ and vapor pressure of 55.57 $\mathrm{kPa}(8.06 \mathrm{psia})$ for cycles 21-30. The vapor pressure of the system increased slightly from cycles $1-10$ to cycles $21-30$. The small increase of $3.87 \mathrm{kPa}(0.56 \mathrm{psi})$ is attributed to the dissolution of the SEI during the 7 hour rest prior to cycle 11. Decomposition and reformation of the SEI has been shown to produce gaseous products in the electrolyte [34], which caused the slight increase in the electrolyte vapor pressure. The chronopotentiogram of Figure 5-40 shows very similar properties to those seen in the previous 20 cycles. There appears to be a small SEI reformation capacity loss during the $21^{\text {st }}$ charge, which had a coulombic efficiency of $75.1 \%$ (Figure 5-35). In subsequent cycles, the coulombic efficiency quickly recovered to $>90 \%$. The cell's return to normal function after the extreme heat flux was applied shows definite promise for the proposed internal TMS. 
The charge-discharge voltage profiles of the $\mathrm{LFP} / \mathrm{Cu}_{2} \mathrm{Sb}$ cell during cycles 10, 20, and 30 are shown in Figure 5-41. The same characteristic voltage profile observed in cycle 10 , is still seen in cycles 20 and 30 . This indicates the electrodes are utilizing the same capacityproducing reactions, despite the multiple reformations of the SEI on the electrode

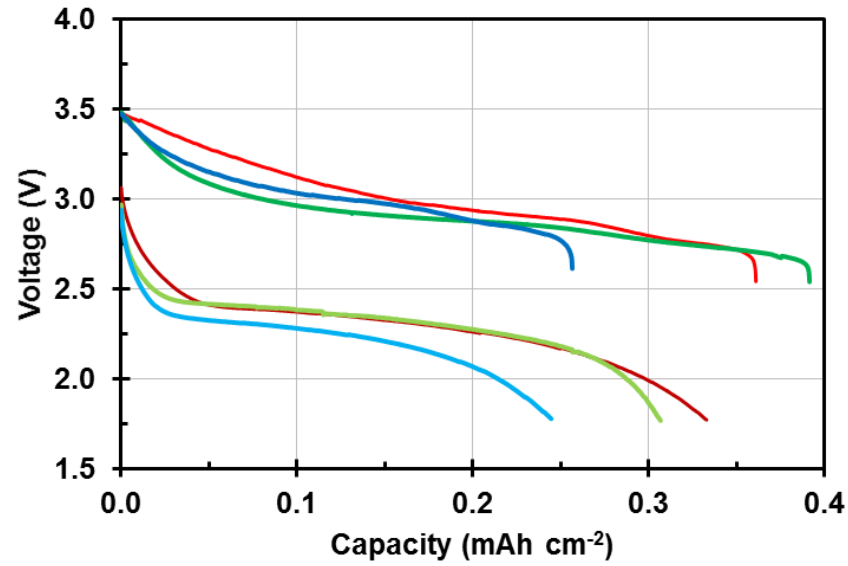

-Charge 10 -Discharge 10-Charge 20

-Discharge 20 - Charge 30 Discharge 30

Figure 5-41: $\mathrm{LFP} / \mathrm{Cu}_{2} \mathrm{Sb}$ Cell Voltage versus Capacity for Cycles 10, 20, and 30

surfaces. The capacity separation of the charge-discharge profiles for each cycle is a direct indication of the coulombic efficiency of the cycle. The lowest coulombic efficiencies of the cell occurred during cycles $11-20$, and the $20^{\text {th }}$ cycle had a coulombic efficiency of $78.4 \%$. The poor coulombic efficiency performance of the cell during boiling was quickly overcome after the heat was removed, rising to $98.9 \%$ during the $25^{\text {th }}$ cycle. The $\mathrm{LFP} / \mathrm{Cu}_{2} \mathrm{Sb}$ cell did experience capacity fade over the 30 cycles; the discharge capacity of the $30^{\text {th }}$ cycle is approximately only $73.5 \%$ of the discharge capacity of the $10^{\text {th }}$ cycle. The binary MFE mixture requires additional refinement to improve its electrochemical performance. Nonetheless, the electrochemical performance demonstrated by the binary mixture under the extreme heat flux is promising.

\subsection{Summary}

The thermal and electrochemical performance of a MFE containing a volatile co-solvent, HFE-7000, has been demonstrated in a boiling facility containing a $\mathrm{LFP} / \mathrm{Cu}_{2} \mathrm{Sb}$ cell. Under continuous vapor generation in the channel of the LFP electrode, the cell was capable of cycling with an average coulombic efficiency of $80 \%$. The coulombic efficiency loss during boiling 
compared to non-boiling, is attributed to $\mathrm{Li}_{3} \mathrm{Sb}$ formation in the $\mathrm{Cu}_{2} \mathrm{Sb}$ anode and continual SEI decomposition at the higher temperatures of the cell. Boiling the MFE is not believed to be a significant source of the capacity fade seen in the cell. The proposed internal TMS that relies on evaporating HFE-7000 from MFE mixture has proven to be feasible. The heat flux applied to the cell to induce the continual vapor generation far exceeded the internal heat generation of a LIB during normal operation. Future investigations into revised cycling voltage limits and SEI stabilizing additives in the MFE are warranted to improve the electrochemical performance of the cell with the proposed TMS. 


\section{CHAPTER 6. CONCLUSION AND RECOMMENDATIONS}

The present study is the first investigation of a MFE for the internal thermal management of LIBs. State-of-the-art TMSs for LIB-powered EVs employ single-phase liquid cooling to the exterior surfaces of the cells within the pack. These systems, although effective at maintaining cell temperatures below those associated with capacity fade and thermal runaway, negatively affect the pack size and weight. In addition, these systems are limited in their effectiveness by the low thermal conductivity of the LIB materials comprising the cell. This can lead to potentially high thermal gradients within the cell, which causes uneven active material utilization and associated aging. The proposed internal TMS eliminates the high thermal gradients that can plague conventional TMSs by the introduction of small vapor generation channels in the positive electrode at strategic locations in the cell. These small channels allow for heat to be quickly dissipated through the evaporation of a volatile co-solvent contained within the MFE mixture. The proposed internal TMS is capable of scaling to address the cooling needs of cells with high capacities and enable cell geometries that are no longer limited by external TMS heat transfer limitations.

Previous investigations to modify LIB electrolytes for their thermal properties have focused on reducing flammability in an effort to improve cell safety. The most flammable component of state-of-the-art electrolyte mixtures are the solvents. Investigators have sought to find alternative co-solvents that produce nonflammable mixtures when mixed with flammable cosolvents in the electrolyte. Some of the co-solvents aimed to significantly reduce the vapor pressure

of the electrolyte to increase thermal stability, while other nonflammable co-solvents were introduced to dominate the vapor phase of the mixture with an inert molecule. The results consistently showed that nonflammable electrolytes can be achieved, but at the expense of electrochemical performance of the LIB. Some of the more promising co-solvents were 
hydrofluoroethers (HFE). Arai formulated a nonflammable electrolyte with HFE-7100 that had comparable electrochemical performance to a conventional electrolyte mixture. The HFE-7000series of fluids all have high vapor pressure, making them ideal for the proposed MFE mixture.

In addition to HFEs, several other co-solvents for the LIB chemistry were proposed and tested, all of which were nonaqueous and aprotic. After basic miscibility testing, the most promising volatile co-solvent for the MFE mixture was HFE-7000. This study is the first to investigate HFE-7000 as a lithium-ion electrolyte co-solvent. The candidate MFE mixture was 1.0 M LiTFSI in 1:1 HFE-7000/EMC by volume. LiTFSI salt was chosen for its high solubility and conductivity in solutions of low dielectric constant solvents. EMC was chosen as the carbonate cosolvent due to the work of Arai who mixed the highest ionic conductivity electrolytes with EMC as compared to other linear carbonate co-solvents. Prior to any coupled thermal and electrochemical testing for the proposed internal TMS with this MFE, the electrochemical performance was compared to a conventional LIB electrolyte mixture: 1.0 $\mathrm{M} \mathrm{LiPF}_{6}$ in 3:7 EC/DEC by weight. The electrochemical experiments performed on the MFE and the baseline electrolyte included conductivity, stability window, half cell cycling and impedance spectroscopy, and full cell cycling and impedance spectroscopy. The results of the conductivity testing showed that the MFE had a lower ionic conductivity than the baseline. This result was not unexpected, and was a consequence of using the organic LiTFSI salt, which has a larger anion and reduces the mobility of lithium-ions in the electrolyte solution. The electrochemical stability window of the electrolytes was measured using cyclic voltammetry with two inert working electrodes, glassy carbon and platinum, with lithium metal counter and reference electrodes. The CV experiment was controlled by a potentiostat. The stability window results with a glassy carbon electrode showed comparable oxidative and reductive decomposition limits for the MFE and baseline electrolyte, and decreased 
reactivity of the MFE between the potential scan limits. The stability window measured with a platinum electrode showed more reactivity for the MFE, but was more likely due to oxygen and water impurities. The oxygen and water impurities are most likely the result of the imperfect process of the freeze-pump-thaw degassing procedure and 3M's HFE-7000 synthesizing process.

Three LIB active materials were used to investigate the electrochemical performance of the electrolytes in half cells: LFP, LTO, and $\mathrm{Cu}_{2} \mathrm{Sb}$. The electrodes used in this study were produced at Prieto Battery using either a slurry-based (LFP, LTO) or an aqueous electrodeposition process $\left(\mathrm{Cu}_{2} \mathrm{Sb}\right)$. The half cells were constructed using $19 \mathrm{~mm}$ PFA T-fittings inside an argon glove box. The cells were galvanostatically cycled using a battery tester at a $0.5 \mathrm{C}$ rate for a total of 10 cycles in an environmental chamber at $25^{\circ} \mathrm{C}$. After these cycles were complete, each cell underwent impedance spectroscopy at the upper and lower voltage limits of the cell. The cell was cycled 10 additional times for a total of 20 cycles, and the impedance measurements were then repeated. The galvanostatic cycling results showed that the MFE produced comparable cycling capacity to the baseline electrolyte in $\mathrm{LTO} / \mathrm{Li}$ and $\mathrm{Cu}_{2} \mathrm{Sb} / \mathrm{Li}$ cells. The impedance data showed that the MFE-containing cells did have a higher cell resistance directly attributed to the lower ionic conductivity of the electrolyte, but had comparable charge transfer resistance and double-layer capacitance. MFE-containing LFP/Li cells did not demonstrate comparable performance to the cell made with the baseline electrolyte. The upper voltage limit of the half cell ( $\left.4 \mathrm{~V} \mathrm{vs} . \mathrm{Li} / \mathrm{Li}^{+}\right)$appeared to activate the aluminum corrosion reaction associated with the TFSI ${ }^{-}$anion of the organic LiTFSI salt. Several published studies have shown different methods of supressing the aluminum corrosion reaction with the LiTFSI salt, and warrant further investigation with the current MFE mixture.

The same three LIB active materials were used to formulate two full cell architectures: $\mathrm{LFP} / \mathrm{Cu}_{2} \mathrm{Sb}$ and LFP/LTO. In both of the full cell architectures, the anode active material limited 
capacity. The same galvanostatic cycling and impedance spectroscopy measurements were made as the half cell experiments. In $\mathrm{LFP} / \mathrm{Cu}_{2} \mathrm{Sb}$ cells, the MFE cycled with higher coulombic efficiency than the baseline throughout all 20 cycles. Both the MFE and baseline cells experienced slight capacity fade that can be attributed to voltage limits of the cell. Because the $\mathrm{Cu}_{2} \mathrm{Sb}$ electrode was the limiting capacity of the cell, its potential versus lithium changed much more significantly than LFP and very likely decreased below $0.5 \mathrm{~V}$ vs. $\mathrm{Li} / \mathrm{Li}^{+}$. The delivered capacity of $\mathrm{LFP} / \mathrm{Cu}_{2} \mathrm{Sb}$ cells was much greater than $\mathrm{Cu}_{2} \mathrm{Sb} / \mathrm{Li}$ cells for similarly manufactured $\mathrm{Cu}_{2} \mathrm{Sb}$ electrodes. This caused excess $\mathrm{Li}_{3} \mathrm{Sb}$ formation and subsequent copper extrusion from the electrode structure. Studies have shown that the reversibility of $\mathrm{Cu}_{2} \mathrm{Sb}$ is compromised when $\mathrm{Li}_{3} \mathrm{Sb}$ is formed, which is the only capacity producing reaction at potentials below $0.5 \mathrm{~V}$ vs. $\mathrm{Li} / \mathrm{Li}^{+}$. Excess $\mathrm{Li}_{3} \mathrm{Sb}$ formation occurred in $\mathrm{LFP} / \mathrm{Cu}_{2} \mathrm{Sb}$ cells independent of the electrolyte. The impedance data showed the MFEcontaining cells had lower charge transfer resistance and higher double-layer capacitance than the baseline cells, but, more importantly, showed that the aluminum corrosion reaction was not active. In LFP/LTO cells, the MFE and baseline showed equal capacity fade over the 20 cycles, suggesting the same capacity degradation mechanism was present for both electrolytes. It is believed that the capacity fade is due to LFP particle cracking due to the entire utilization of its available capacity with the full cell voltage limits selected. The impedance spectra for both electrolytes showed increasing charge transfer resistance, which can be attributed to continually re-passivation of the newly exposed surfaces of the cracked LFP particles. In both LFP/Cu $\mathrm{Sb}_{2} \mathrm{Sb}$ and LFP/LTO cells, the MFE performed comparably well to the baseline electrolyte. Investigation into revised full cell voltage limits are warranted to reduce the amount of capacity fade observed from excess $\mathrm{Li}_{3} \mathrm{Sb}$ formation and LFP particle cracking. 
After the electrochemical performance of the MFE was established, the coupled thermalelectrochemical performance of the MFE was investigated. To prove the feasibility of the proposed TMS, the electrochemical cycling of a full cell with the MFE needed to be demonstrated while the volatile co-solvent (HFE-7000) continuously boiled. This experiment required the development and construction of a custom electrolyte boiling facility. The facility contained a $\mathrm{LFP} / \mathrm{Cu}_{2} \mathrm{Sb}$ cell that enabled direct viewing of a vapor generation channel contained in the LFP positive electrode. A small thin film heater was placed on the backside of the LFP electrode to induce continuous vapor generation in the channel. The test facility was capable of measuring the heater temperature, bulk electrolyte temperature, condenser temperatures, and system vapor pressure. During testing, $4 \mathrm{~W}$ of heat input was sufficient to continuously evaporate the MFE in the channel. The $\mathrm{LFP} / \mathrm{Cu}_{2} \mathrm{Sb}$ cell was galvanostatically cycled for a total of 30 cycles: the first 10 without heat input, the second 10 with a $4 \mathrm{~W}$ heat input, and the final 10 without heat input.

The results showed that the cell was capable of cycling under continuous vapor generation with an average coulombic efficiency of $80 \%$. The cell was observed to self-discharge while the system was warmed with the $4 \mathrm{~W}$ heat input during the 7 hour rest period prior to cycling. It was believed that the increased temperature reduced the stability of the SEI layer, causing increased reactivity at both the anode and cathode interfaces. With the addition of SEI stabilizing additives to the MFE mixture, the thermal stability of the SEI is expected to significantly improve. The slight capacity fade seen in the cell appeared to be consistent with the capacity fade seen in the $\mathrm{LFP} / \mathrm{Cu}_{2} \mathrm{Sb} 19 \mathrm{~mm}$ PFA T-fitting cells, indicating excess formation of $\mathrm{Li}_{3} \mathrm{Sb}$ in the capacitylimiting $\mathrm{Cu}_{2} \mathrm{Sb}$ anode. The cell voltage limits between the two experiments (PFA cells and EBF) were unchanged. This source of capacity fade appeared to be the only one present: boiling the electrolyte continuously did not adversely affect the cell. Most promisingly, the heat flux applied 
to the cell to induce the continuous vapor generation far exceeded the internal heat generation of a LIB during normal operation. This result showed definite promise for the proposed internal TMS. Continuous vapor generation in the LIB is not expected to occur under normal operating conditions; therefore, the excess heat rejection capability of the proposed internal TMS could provide safety improvements over external TMSs by removing heat from the normally insulated internal portion of the cell during a thermal event (e.g., an internal short). The experiments performed in the EBF were the first of their kind: no studies have ever reported evaporating a cosolvent in the electrolyte while cycling a LIB cell. The experiments proved that boiling the electrolyte is possible without salt precipitation under the conditions of the experiment in the present study and appeared to have a relatively small impact on the electrochemical performance of the $\mathrm{LFP} / \mathrm{Cu}_{2} \mathrm{Sb}$ cell.

\subsection{Recommendations for Future Research}

The current study has answered many fundamental questions towards using the electrolyte as part of an internal TMS for a LIB. However, significant work is required to continue to develop the proposed TMS for its ultimate deployment in a large LIB pack:

- The binary MFE mixture requires refinement to continue to improve its electrochemical and thermal performance. The mixture currently contains no solvents or additives commonly credited for the creation of a stable SEI. Such solvents and additives include ethylene carbonate, vinylene carbonate, fluoroethylene carbonate, and $\mathrm{LiPF}_{6}$. Furthermore, the relative amount of HFE-7000 in the MFE mixture is not optimized. A 1:1 mixing ratio by volume of EMC to HFE-7000 was chosen only as a starting point. Future work can assess the thermal and electrochemical impact of reducing or increasing the amount of HFE-7000 relative to the other solvents. With a reduction in HFE-7000, it will be possible 
to mix EC to much higher concentrations than Arai showed in his work [86]. Higher concentrations of EC might provide the opportunity to exclusively use $\mathrm{LiPF}_{6}$ as the electrolyte salt, and forego the lower ionic conductivity and aluminum corrosion issues of LiTFSI. The impact of the MFE mixture refinement can be assessed using the experimental techniques and facilities developed in this study.

- The electrochemical performance of the MFE needs to be evaluated with other, higher energy LIB active materials to be comparable to the cell chemistries currently employed in EVs. LFP was chosen as a positive electrode material for its relatively low redox potential versus $\mathrm{Li} / \mathrm{Li}^{+}$, which precluded the need to form a substantial SEI. Higher energy cathode active materials such as lithium nickel cobalt aluminum oxide, lithium nickel manganese cobalt oxide, and lithium manganese oxide warrant investigation with the MFE. Furthermore, $\mathrm{LTO}$ and $\mathrm{Cu}_{2} \mathrm{Sb}$ were used as alternatives to using a graphite negative electrode, both of which have a higher redox potential versus $\mathrm{Li} / \mathrm{Li}^{+}$. Graphite was specifically avoided in this study due to the lack of SEI stabilizing additives in the binary MFE mixture required for successful passivation of graphite. However, graphite is the state-of-the-art negative electrode material and needs to be evaluated with the MFE. If a refined MFE mixture is developed that includes SEI stabilizing additives (e.g., EC and $\mathrm{LiPF}_{6}$ ), its electrochemical performance needs to be evaluated with graphite. The combination of a higher energy cathode and anode with the MFE will produce a state-ofthe-art LIB that can fundamentally change how LIB thermal management is approached.

- The current study did not include any thermal modeling efforts of a LIB containing evaporation channels in the positive electrode. The modeling study needs to simulate (1) normal volumetric heat generation of a LIB with vapor generation channels and (2) thermal 
runaway in an immediately adjacent with simultaneous conduction through the battery and convective heat rejection in vapor generation channels. The results of thermal modeling efforts can better inform the ideal number and placement of evaporation channels in the positive electrode to minimize thermal gradients throughout the electrode stack.

- In the present study, the vapor generation channel in the positive electrode was created by abrasively removing the electrode coating with a metal spatula. Although it worked well to quickly produce the channel in this study, this is not a scalable or economical manufacturing process. Therefore, the development of a scalable vapor generation channel manufacturing process for slurry-based positive electrodes is a critical need for the future development of the proposed internal TMS.

- The final recommendation from this study is to demonstrate the system concept in a higher capacity cell (>1 Ah) with a more realistic MFE volumetric fill. The EBF was designed to minimize the required volumetric fill of the MFE; however, $75 \mathrm{~mL}$ of electrolyte is an extreme excess for the size of cell studied in this work. This concept also needs to package the condenser to a more commercially-viable size. Under normal heat generation, the surface of the cell casing should be sufficient in providing adequate heat transfer area to condense the evaporated HFE-7000. With a successful system demonstration, the proposed internal TMS can be directly compared to conventional TMS in EVs and other applications to assess the system volumetric and gravimetric energy density improvements. 


\section{REFERENCES}

1. T. B. R. David Linden, Linden's Handbook of Batteries, McGraw-Hill (2011).

2. E. E. Donald Chung, Shriram Santhanagopalan, Automotive Lithium-ion Battery (LIB) Supply Chain and U.S. Competitiveness Considerations, Clean Energy Manufacturing Analysis Center (2015).

3. T. M. Bandhauer, S. Garimella and T. F. Fuller, Journal of the Electrochemical Society, 158, R1 (2011).

4. R. Saidur, M. Rezaei, W. K. Muzammil, M. H. Hassan, S. Paria and M. Hasanuzzaman, Renewable and Sustainable Energy Reviews, 16, 5649 (2012).

5. G. Pistoia, Lithium-Ion Batteries: Advances and Applications, Elsevier, USA (2014).

6. T. R. Board and N. R. Council, Overcoming Barriers to Deployment of Plug-in Electric Vehicles, p. 152, The National Academies Press, Washington, DC (2015).

7. G. Girishkumar, B. McCloskey, A. C. Luntz, S. Swanson and W. Wilcke, The Journal of Physical Chemistry Letters, 1, 2193 (2010).

8. T. Network, Tesla Model S Weight Distribution, in Model S (2013).

9. U. S. E. I. Administration, Renewable \& Alternative Fuels, in (2016).

10. U. S. E. I. Administration, Consumption \& Efficiency, in (2016).

11. W. Xu, J. Wang, F. Ding, X. Chen, E. Nasybulin, Y. Zhang and J.-G. Zhang, Energy \& Environmental Science, 7, 513 (2014).

12. T. Ohzuku, Y. Iwakoshi and K. Sawai, Journal of The Electrochemical Society, 140, 2490 (1993).

13. K. Takahashi and V. Srinivasan, Journal of The Electrochemical Society, 162, A635 (2015).

14. T. Ohzuku, A. Ueda and N. Yamamoto, Journal of The Electrochemical Society, 142, 1431 (1995).

15. M. Winter and J. O. Besenhard, Electrochimica Acta, 45, 31 (1999).

16. B. Liang, Y. Liu and Y. Xu, Journal of Power Sources, 267, 469 (2014).

17. L. M. L. Fransson, J. T. Vaughey, R. Benedek, K. Edström, J. O. Thomas and M. M. Thackeray, Electrochemistry Communications, 3, 317 (2001). 
18. M. Hu, X. Pang and Z. Zhou, Journal of Power Sources, 237, 229 (2013).

19. J. B. Goodenough and Y. Kim, Chemistry of Materials, 22, 587 (2009).

20. M. B. Materials, Powering the Future, 3M (2011).

21. R. Matthe, L. Turner and H. Mettlach, SAE Int. J. Engines, 4, 1944 (2011).

22. K. Xu, Chemical Reviews (2014).

23. K. X. M. S. Ding, S. S. Zhang, K. Amine, G. L. Henriksen, T. R. Jow, Journal of the Electrochemical Society, 148, A1196 (2001).

24. K. Xu, Chemistry Review, 104, 4303 (2004).

25. D. O'Hagan, Chemical Society Reviews, 37, 308 (2008).

26. P. Arora and Z. Zhang, Chemical Reviews, 104, 4419 (2004).

27. V. Deimede and C. Elmasides, Energy Technology, 3, 453 (2015).

28. J. Trevey, J. S. Jang, Y. S. Jung, C. R. Stoldt and S.-H. Lee, Electrochemistry Communications, 11, 1830 (2009).

29. L. F. Allen Bard, Electrochemical Methods: Fundamentals and Applications, John Wiley $\&$ Sons, New York (2001).

30. U. Seong Kim, J. Yi, C. B. Shin, T. Han and S. Park, Journal of The Electrochemical Society, 158, A611 (2011).

31. T. B. R. David Linden, Handbook of Batteries, McGraw-Hill (2002).

32. M. Griggs, 'Solar Impulse 2' Delayed By Battery Troubles, in Aviation, Popular Science (2015).

33. B. A. Johnson and R. E. White, Journal of Power Sources, 70, 48 (1998).

34. Q. Wang, P. Ping, X. Zhao, G. Chu, J. Sun and C. Chen, Journal of Power Sources, 208, 210 (2012).

35. N. T. S. Board, Incident Report: Auxiliary Power Unit Battery Fire, in (2014).

36. M. Sinnett, Moving Forward With the 787, Boeing (2013).

37. P. D. Rawlinson, Vehicle battery pack ballistic shield, Google Patents (2013).

38. B. Smith, Chevrolet Volt Battery Incident Summary Report, NHSTA Editor, Office of Vehicle Safety Compliance, Washington, DC (2012). 
39. C. Jensen, Chevy Volt Fire Prompts Federal Investigation Into Lithium-Ion Batteries, in The New York Times (2011).

40. C. Jensen, Tesla Says Car Fire Started in Battery, in The New York Times (2013).

41. E. Musk, Model S Fire, in Tesla Updates, Tesla (2013).

42. W. Godfrey, Fire - Propulsion Battery - Road Debris, in Office of Defects Investigation (2014).

43. E. Musk, Tesla Adds Titanium Underbody Shield and Aluminum Deflector Plates to Model $\mathrm{S}$, in Tesla Updates, Tesla (2014).

44. W. Freedman, Child's Hoverboard Explodes, Catches Fire Inside Pentaluma Home, in $A B C$ 7 News, California (2016).

45. C. News, Fire explosions linked to e-cigarettes spark safety concerns, in CBS (2016).

46. C. Mikolajczak, M. Kahn, K. White and R. T. Long, Lithium-Ion Batteries Hazard and Use Assessment, Springer US (2011).

47. I. Buchmann, BU-301a: Types of Battery Cells, Battery University (2015).

48. wk057, Pics/Info: Inside the battery pack, in Model S: Battery \& Charging, Tesla Motors Club (2014).

49. Nissan, Charging and Range, in 2015 LEAF, Nissan (2015).

50. T. Williams, All The Results From Independent Test Of Nissan LEAFs With Lost Capacity. Not All Instrument Failure, InsideEVs (2012).

51. J. Cole, Nissan Says Capacity Loss Issues Are Due To High Mileage. Pheonix Cars Are Still Expected to Retain 76\% After 5 Years, InsideEVs (2012).

52. N. W. Service, Pheonix, AZ Monthly Weather Summary, 2014 ed., National Weather Service Forecast Office (2014).

53. P. Liu, J. Wang, J. Hicks-Garner, E. Sherman, S. Soukiazian, M. Verbrugge, H. Tataria, J. Musser and P. Finamore, Journal of The Electrochemical Society, 157, A499 (2010).

54. J. Cole, Nissan CEO Carlos Ghosn: Second Generation Battery Is Coming Online NOW, InsideEVs (2012).

55. S. Panchal, S. Mathewson, R. Fraser, R. Culham and M. Fowler, SAE Int. J. Alt. Power., 4, 293 (2015).

56. E. B. Center, Nissan Leaf Batteries, Hybrid Auto Center (2015). 
57. M. LaMonica, Are Air-Cooled Batteries Hurting Nissan Leaf Range?, MIT Technology Review (2012).

58. A. Prilutsky and W. A. Hermann, Active Thermal Runaway Mitigation System for Use Within a Battery Pack, Google Patents (2010).

59. W. A. Hermann, Liquid cooling manifold with multi-function thermal interface, Google Patents (2012).

60. V. H. Mehta and A. Prilutsky, Cell thermal runaway propagation resistance using dual intumescent material layers, Google Patents (2010).

61. V. H. Mehta, A. Prilutsky and W. A. Hermann, Cell with an outer layer of intumescent material, Google Patents (2012).

62. R. Parrish, K. Elankumaran, M. Gandhi, B. Nance, P. Meehan, D. Milburn, S. Siddiqui and A. Brenz, Voltec Battery Design and Manufacturing, SAE International (2011).

63. K. Schultz, Chevy Volt Batteries, in Delta-11 - Project Overview, Schultz Engineering (2015).

64. H. S. Hamut, I. Dincer and G. F. Naterer, Journal of Energy Resources Technology, 136, 011202 (2014).

65. B. M. Conlon, T. Blohm, M. Harpster, A. Holmes, M. Palardy, S. Tarnowsky and L. Zhou, SAE Int. J. Alt. Power., 4, 248 (2015).

66. F. Markus, 2016 Chevrolet Volt Technical First Look, in Motor Trend, The Enthusiast Network (2014).

67. PHEV Battery Testing Results: 2013 Chevrolet Volt - VIN 3491, Idaho National Laboratory (2015).

68. PHEV Battery Testing Results: 2013 Chevrolet Volt - VIN 3929, Idaho National Laboratory (2015).

69. BEV Battery Testing Results: 2013 Nissan Leaf S - VIN 7885, Idaho National Laboratory (2015).

70. E. V. Wiki, Battery Specs, Electric Vehicle Wiki (2010).

71. G. C. Congress, Panasonic Develops New Higher-Capacity 18650 Li-Ion Cells; Application of Silicon-based Alloy in Anode, Green Car Congress (2009).

72. T. M. Bandhauer and S. Garimella, Applied Thermal Engineering, 61, 756 (2013).

73. S. K. Mohammadian, Y.-L. He and Y. Zhang, Journal of Power Sources, 293, 458 (2015). 
74. J. K. P. S. E. Sloop, S. Wang, J. B. Kerr, and K. Kinoshita, Electrochemical and SolidState Letters, 4, A42 (2001).

75. K. F. Ganesan Nagasubramanian, Electrochimica Acta, 101, 3 (2013).

76. X. Wang, E. Yasukawa and S. Kasuya Journal of The Electrochemical Society, 148, A1058 (2001).

77. M. S. D. Kang Xu, Shengshui Zhang, Jan L. Allen, T. Richard Jow, Journal of the Electrochemical Society, 149, A1079 (2002).

78. M. S. D. Kang Xu, Shengshui Zhang, Jan L. Allen, T. Richard Jow, Journal of the Electrochemical Society, 150, A161 (2003).

79. M. S. D. Kang Xu, Shengshui Zhang, Jan L. Allen, T. Richard Jow, Journal of the Electrochemical Society, 150, A170 (2003).

80. S. S. Zhang, K. Xu and T. R. Jow, Journal of Power Sources, 113, 166 (2003).

81. D. H. C. Wong, J. L. Thelen, Y. Fu, D. Devaux, A. A. Pandya, V. S. Battaglia, N. P. Balsara and J. M. DeSimone, Proceedings of the National Academy of Sciences, 111, 3327 (2014).

82. Solvay, Galden HT PFPE, in Heat Transfer Fluids, Solvay (2014).

83. W.-T. Tsai, Journal of Hazardous Materials, 119, 69 (2005).

84. J. Arai, Lithium Secondary Battery and Liquid Electrolyte for the Battery, H01M 4/58 ed., Hitachi, Ltd. (2001).

85. J. Arai, Journal of Applied Electrochemistry, 32, 1071 (2002).

86. J. Arai, Journal of the Electrochemical Society, 150, A219 (2003).

87. J. Arai, Journal of Power Sources, 119-121, 388 (2003).

88. 3M, 3M Thermal Management Fluids, in (2009).

89. M. S. Ding and T. R. Jow, Journal of The Electrochemical Society, 152, A1199 (2005).

90. E. I. Katsuhiko Naoi, Nobuhiro Ogihara, Yasuhiro Nakamura, Haruki Segawa, Yuji Ino, Journal of the Electrochemical Society, 156, A272 (2009).

91. E. I. Katsuhiko Naoi, Yoki Honda, and Fumitaka Shimodate, Journal of the Electrochemical Society, 157, A190 (2010).

92. C. J. O. Ganesan Nagasubramanian, Journal of Power Sources, 196, 8604 (2011).

93. S. H. Noie, Applied Thermal Engineering, 25, 495 (2005). 
94. D. Aurbach, Y. Talyosef, B. Markovsky, E. Markevich, E. Zinigrad, L. Asraf, J. S. Gnanaraj and H.-J. Kim, Electrochimica Acta, 50, 247 (2004).

95. R. Jow, K. Xu, O. Borodin and M. Ue, Electrolytes for Lithium and Lithium-Ion Batteries, p. 476, Springer-Verlag, New York (2014).

96. C.-K. Park, Z. Zhang, Z. Xu, A. Kakirde, K. Kang, C. Chai, G. Au and L. Cristo, Journal of Power Sources, 165, 892 (2007).

97. U. Heider, R. Oesten and M. Jungnitz, Journal of Power Sources, 81-82, 119 (1999).

98. J. S. Wright, D. J. Carpenter, D. J. McKay and K. U. Ingold, Journal of the American Chemical Society, 119, 4245 (1997).

99. J. L. Franklin and H. E. Lumpkin, Journal of the American Chemical Society, 74, 1023 (1952).

100. A.-A. G. Shaikh and S. Sivaram, Chemical Reviews, 96, 951 (1996).

101. D. P. W. J. T. Dudley, G. Thomas, R. LeVae, S. Woo, H. Blom, C. Horvath, M.W. Juzkow, B. Denis, P. Juric, P. Aghakian, J. R. Dahn, Journal of Power Sources, 35, 59 (1991).

102. P. Verma, P. Maire and P. Novák, Electrochimica Acta, 55, 6332 (2010).

103. G. Lamoureux, A. D. MacKerell and B. t. Roux, The Journal of Chemical Physics, 119, 5185 (2003).

104. A. K. Rappe and W. A. Goddard, The Journal of Physical Chemistry, 95, 3358 (1991).

105. M. S. Ding, Journal of the Electrochemical Society, 150, A455 (2003).

106. M. Morita, T. Shibata, N. Yoshimoto and M. Ishikawa, Electrochimica Acta, 47, 2787 (2002).

107. M. Morita, T. Shibata, N. Yoshimoto and M. Ishikawa, Journal of Power Sources, 119121, 784 (2003).

108. P. T. Kissinger and W. R. Heineman, Journal of Chemical Education, 60, 702 (1983).

109. G. A. Mabbott, Journal of Chemical Education, 60, 697 (1983).

110. J. J. Van Benschoten, J. Y. Lewis, W. R. Heineman, D. A. Roston and P. T. Kissinger, Journal of Chemical Education, 60, 772 (1983).

111. D. Aurbach, M. Daroux, P. Faguy and E. Yeager, Journal of Electroanalytical Chemistry and Interfacial Electrochemistry, 297, 225 (1991).

112. V. Borgel, E. Markevich, D. Aurbach, G. Semrau and M. Schmidt, Journal of Power Sources, 189, 331 (2009). 
113. M. Nagahama, N. Hasegawa and S. Okada, Journal of The Electrochemical Society, 157, A748 (2010).

114. S. Liu, N. Imanishi, T. Zhang, A. Hirano, Y. Takeda, O. Yamamoto and J. Yang, Journal of Power Sources, 195, 6847 (2010).

115. Z. Zhang, L. Hu, H. Wu, W. Weng, M. Koh, P. C. Redfern, L. A. Curtiss and K. Amine, Energy \& Environmental Science, 6, 1806 (2013).

116. BASi, Polishing Guidelines, Bioanalytical Systems (2015).

117. X. Zhang, R. Kostecki, T. J. Richardson, J. K. Pugh and P. N. Ross, Journal of The Electrochemical Society, 148, A1341 (2001).

118. L.-X. Yuan, Z.-H. Wang, W.-X. Zhang, X.-L. Hu, J.-T. Chen, Y.-H. Huang and J. B. Goodenough, Energy \& Environmental Science, 4, 269 (2011).

119. A. K. Padhi, K. S. Nanjundaswamy, C. Masquelier, S. Okada and J. B. Goodenough, Journal of The Electrochemical Society, 144, 1609 (1997).

120. S.-L. Chou, J.-Z. Wang, H.-K. Liu and S.-X. Dou, The Journal of Physical Chemistry C, 115, 16220 (2011).

121. L. Kavan and M. Grätzel, Electrochemical and Solid-State Letters, 5, A39 (2002).

122. J. R. M. Evgenij Barsoukov, Impedance Spectroscopy: Theory, Experiment, and Applications, Wiley-Interscience, New Jersey (2005).

123. M. E. Orazem, Electrochemical impedance spectroscopy, Wiley, Hoboken, N.J. (2008).

124. A. V. Oppenheim, A. S. Willsky and S. H. Nawab, Signals \& Systems (2nd ed.), p. 957, Prentice-Hall, Inc. (1996).

125. G. J. Brug, A. L. G. van den Eeden, M. Sluyters-Rehbach and J. H. Sluyters, Journal of Electroanalytical Chemistry and Interfacial Electrochemistry, 176, 275 (1984).

126. M. E. Orazem, I. Frateur, B. Tribollet, V. Vivier, S. Marcelin, N. Pébère, A. L. Bunge, E. A. White, D. P. Riemer and M. Musiani, Journal of The Electrochemical Society, 160, C215 (2013).

127. J. M. Mosby and A. L. Prieto, Journal of the American Chemical Society, 130, 10656 (2008).

128. S.-W. Song, R. P. Reade, E. J. Cairns, J. T. Vaughey, M. M. Thackeray and K. A. Striebel, Journal of The Electrochemical Society, 151, A1012 (2004).

129. G. Yan, X. Li, Z. Wang, H. Guo and J. Wang, The Journal of Physical Chemistry C, 118, $6586(2014)$. 
130. K. Wu, J. Yang, X.-Y. Qiu, J.-M. Xu, Q.-Q. Zhang, J. Jin and Q.-C. Zhuang, Electrochimica Acta, 108, 841 (2013).

131. N. Takami, K. Hoshina and H. Inagaki, Journal of The Electrochemical Society, 158, A725 (2011).

132. X. Yan, W. Liu, W. Yan, D. Sun, Y. Jin, J. Wang, L. Xiang, H. Munakata and K. Kanamura, Electrochimica Acta, 191, 661 (2016).

133. S. Matsuno, M. Noji, T. Kashiwagi, M. Nakayama and M. Wakihara, The Journal of Physical Chemistry C, 111, 7548 (2007).

134. J. Ren, X. He, W. Pu, C. Jiang and C. Wan, Electrochimica Acta, 52, 1538 (2006).

135. H. Bryngelsson, J. Eskhult, L. Nyholm and K. Edström, Electrochimica Acta, 53, 7226 (2008).

136. D. Aurbach, B. Markovsky, G. Salitra, E. Markevich, Y. Talyossef, M. Koltypin, L. Nazar, B. Ellis and D. Kovacheva, Journal of Power Sources, 165, 491 (2007).

137. D. Wang, X. Wu, Z. Wang and L. Chen, Journal of Power Sources, 140, 125 (2005).

138. M. Dahbi, F. Ghamouss, F. Tran-Van, D. Lemordant and M. Anouti, Journal of Power Sources, 196, 9743 (2011).

139. X. Chen, W. Xu, M. H. Engelhard, J. Zheng, Y. Zhang, F. Ding, J. Qian and J.-G. Zhang, Journal of Materials Chemistry A, 2, 2346 (2014).

140. K. Dokko, N. Tachikawa, K. Yamauchi, M. Tsuchiya, A. Yamazaki, E. Takashima, J.-W. Park, K. Ueno, S. Seki, N. Serizawa and M. Watanabe, Journal of The Electrochemical Society, 160, A1304 (2013).

141. H. Moon, T. Mandai, R. Tatara, K. Ueno, A. Yamazaki, K. Yoshida, S. Seki, K. Dokko and M. Watanabe, The Journal of Physical Chemistry C, 119, 3957 (2015).

142. R. E. Sonntag and G. J. Wylen, in Introduction to Thermodynamics: Classical and Statistical, 3rd ed., p. 479, John Wiley, New York (1991).

143. X. Zhang, J. Zuo and C. Jian, Journal of Chemical \& Engineering Data, 55, 4896 (2010). 


\section{APPENDIX A. FREEZE-PUMP-THAW LIQUID DEGASSING PROCEDURE}

The following freeze-pump-thaw degassing procedure was used for all LIB electrolyte solvents, including HFE-7000 and EMC. It was also used on Perflenapent and FC-72 prior to their miscibility testing with EMC. Aside from LIB electrolyte solvents, it is recommended that any fluids that enter the argon glove box undergo the freeze-pump-thaw degassing procedure. The only limitation for the use of the procedure is the boiling temperature of liquid nitrogen, $77 \mathrm{~K}$. Any fluids that are to be degassed must have a freezing point greater than $77 \mathrm{~K}$.

1. Clean and rinse a Schlenk flask and cap with an appropriate solvent; typically, de-ionized water is suitable.

2. Vacuum dry the Schlenk flask without the PTFE cap threaded in place overnight in the oven at $80^{\circ} \mathrm{C}$.

3. Insert the solvent to be degassed in the flask using a funnel. Be sure to leave adequate head room in the flask, especially if it is uncertain if the fluid will expand when solidified. Ensure the cap is properly sealed: a white ring becomes visible in the glass stem when the cap has properly seated (Figure A-1).

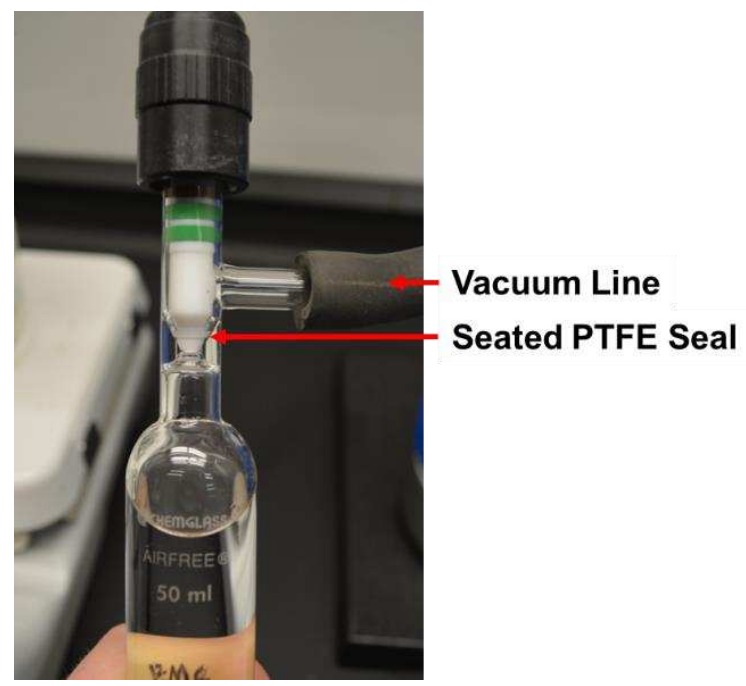

Figure A-1: $50 \mathrm{~mL}$ Sealed Schlenk Flask Connected to Vacuum Line

4. Connect the Schlenk flask to a vacuum line.

The lab fume hood has a three line vacuum manifold (Figure A-2). 
5. Fill the two dewars noted in Figure A-2 with liquid nitrogen. Wrap a rag around the top of the dewar to minimize the amount of liquid nitrogen loss. Turn on the vaccum pump, but do not open any vacuum valves at this time.

6. Submerge the Schlenk flask

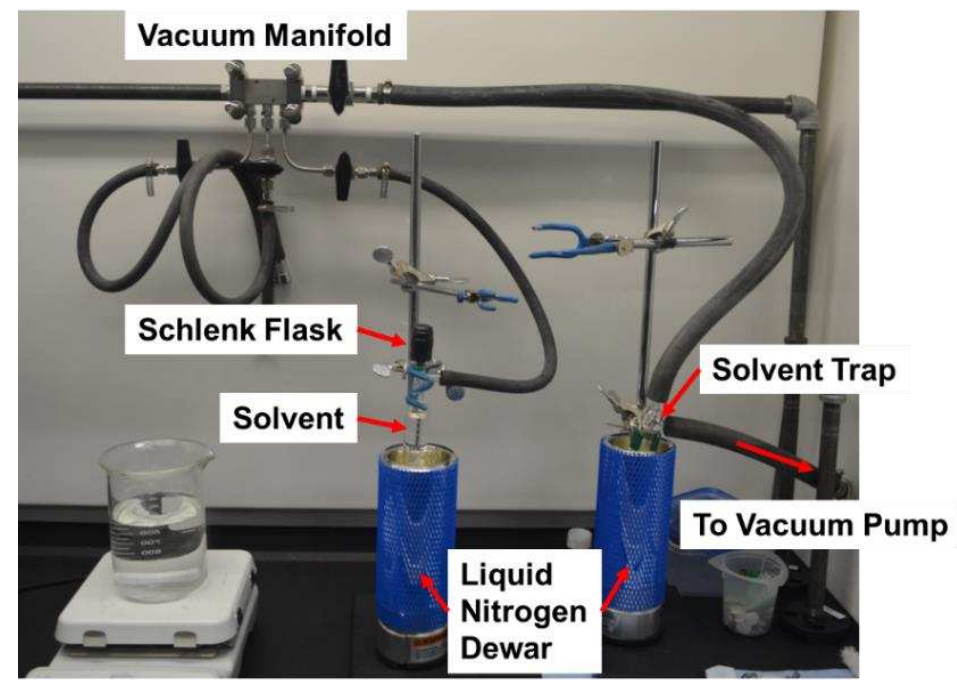

Figure A-2: Freeze-Pump-Thaw Vacuum Manifold Setup in Fume Hood into the liquid nitrogen dewar. Secure the flask in place with a ring stand clamp. Allow sufficient time for the liquid contents to freeze solid.

7. Once the contents are frozen solid, open the main vacuum manifold valve. Then, open the next downstream valve. The vacuum line connected to the flask should now be under vacuum. Open the Schlenk cap for the headspace above the frozen liquid to be evacuated. Leave the Schlenk flask submerged in the liquid nitrogen throughout the pumping process.

8. After 10-20 minutes, tighten the Schlenk cap and remake the seal. Leave all the vacuum valves open.

9. Leaving the vacuum line in place, remove the flask from the dewar and carefully submerge in a luke-warm water bath to thaw the solvent (Figure A-3). A heated stir plate is ideal for maintaining a uniform temperature water bath. Make sure the entire frozen section of the flask is submerged in the beaker to minimize severe temperature gradients which can cause the flask to shatter. Watch for gas evolution as the frozen solvent thaws. The trapped gas will travel to the headspace of the flask. 
10. Repeat steps 6-9 until no more gas evolution is observed during the thawing process. Repeat the process a minimum of three times.

11. Once completed, close all vacuum valves, disconnect the vacuum line from the flask, and turn off the vacuum pump.

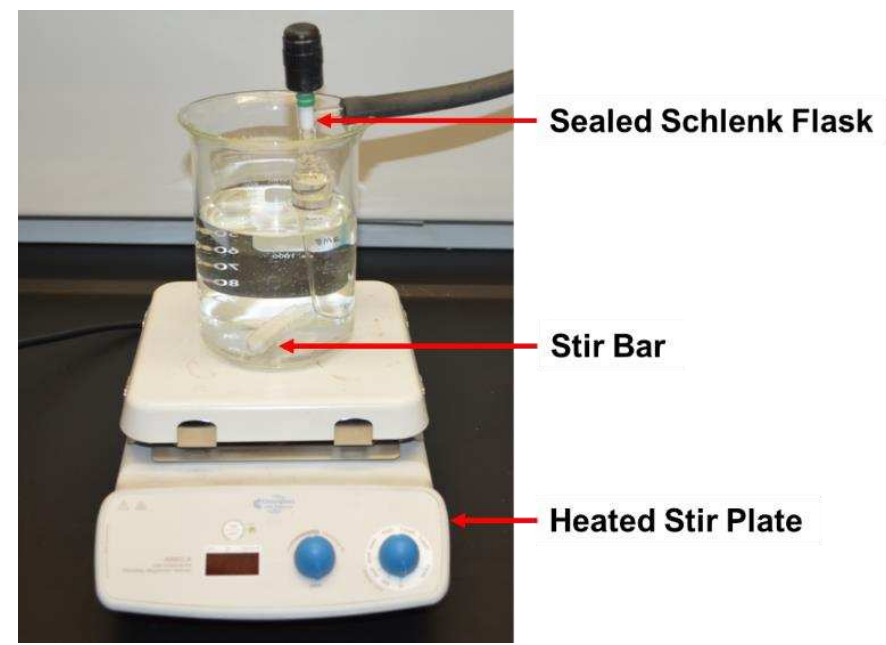

Figure A-3: Luke Warm Water Bath for Thawing Frozen Solvents 


\section{APPENDIX B. WORKING ELECTRODE POLISHING PROCEDURE}

The working electrode polishing procedure presented here is adapted from the polishing procedure provided by BASi [116]. The polishing procedure aims to completely clean the electrode surface which can form many different contaminants during experimentation and sitting in open air for a long duration. This complete procedure was performed on all platinum working electrodes prior to all electrochemical stability window measurements. The glassy carbon working electrode polishing procedure is an abbreviated version of the procedure presented below; only perform steps 1, 4-5 to complete the polishing process. The BASi electrode polishing kit is used.

1. Wearing a pair of clean nitrile gloves, rinse the electrode surface with deionized water followed by methanol. Wipe the electrode dry with a Kimwipe laboratory tissue.

2. Wet the surface of the nylon disk with deionized water and apply a few drops of the $1 \mu \mathrm{m}$ diamond polish slurry on the white nylon disk. Thoroughly shake to mix the diamond slurry prior to applying it to the nylon disk.

3. Using very light and uniform pressure, place the electrode against the nylon disk and move the electrode through the wetted portion in a figureeight motion. Ensure the electrode remains perpendicular to the surface and rotate the electrode in regular 90 degree intervals (Figure B-1). After 12 minutes, remove the electrode from

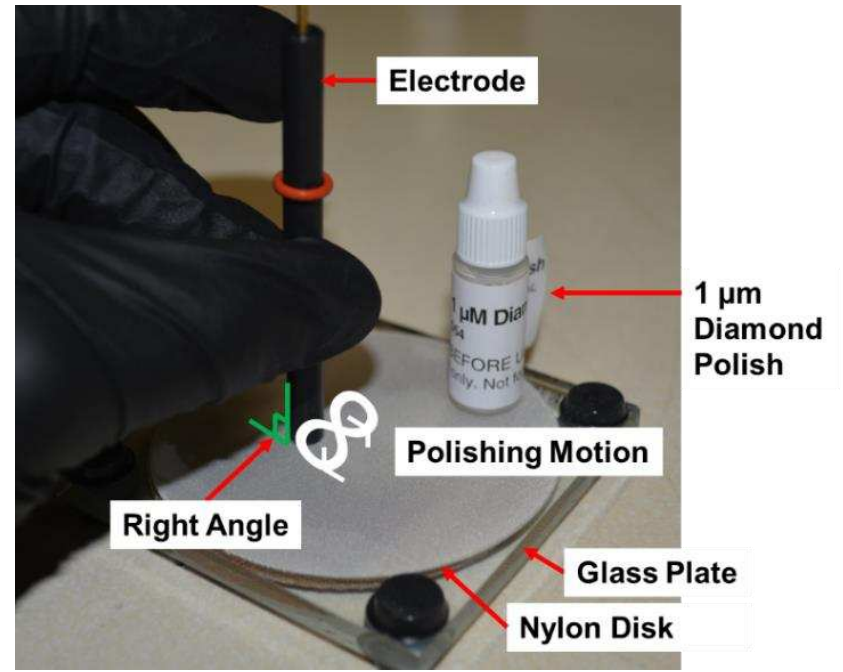

Figure B-1: Platinum Electrode Polishing on Nylon Disk

the nylon disk and rinse with methanol. 
4. Wet the brown microcloth disk surface with deionized water. After thoroughly shaking, apply several drops of the alumina polish (Figure B-2). Use the same procedure described in step 3. After 1-2 minutes, remove the electrode from the

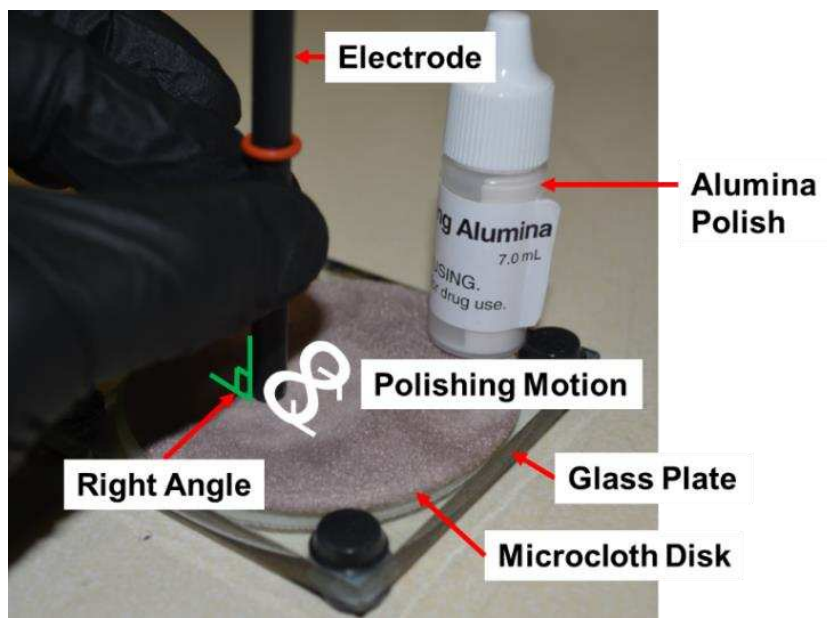
Figure B-2: Electrode Polishing on Microcloth microcloth disk and rinse with Disk deionized water.

5. Rinse the electrode with methanol and dry with a Kimwipe. Do not touch the electrode surface as this can contaminate it or possibly scratch the surface. 


\section{APPENDIX C. SLURRY-BASED ELECTRODE COATING PROCEDURE}

The following slurry-based electrode coating procedure was used to produce the LFP and LTO single-sided electrodes used in half and full cell testing. In addition, this procedure was used for producing the LFP electrode used in the electrolyte boiling facility. All of the electrodes in this work were created on equipment at Prieto Battery (Figure C-1).

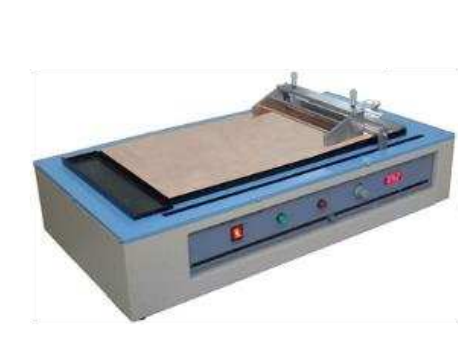

Drawdown Machine

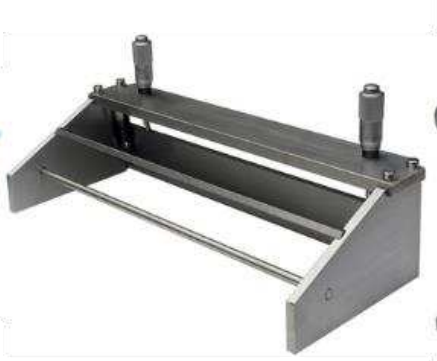

Doctor Blade

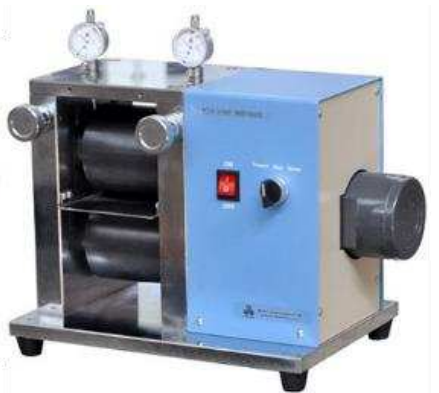

Calendaring Machine

Figure C-1: Surry-Based Electrode Coating Equipment used at Prieto Battery

\section{Initial Current Collector Cutting and Characterization:}

1. Cut strips of copper or aluminum current collector appropriate for the desired application.

2. Label the top of each current collector strip with a sharpie for identification purposes (e.g., “LTO 1”).

3. Measure the weight of each current collector strip and record. This will be used for the determination of the active material loading after the coating process has been completed.

\section{Slurry Mixing:}

1. Measure required weights of PVDF binding agents (Kynar 161 and 761) using a weigh boat and scale and insert into plastic jar.

2. Measure required weight of NMP solvent and insert into plastic jar containing PVDF binding agents. 
3. Place $\sim 30$ stainless steel ball bearings into plastic jar (Figure C-2). Seal lid tightly.

4. Place the jar in the paint can shaking machine (Figure C-3). Secure tightly. Shake for 15 minutes.

5. After shaking, remove the plastic jar. Measure out appropriate quantities of carbon black and active material and place into plastic jar (Figure C-4 and Figure C-5).

6. Place the jar in the paint can shaking machine. Secure tightly. Shake for 15 minutes.

7. The slurry should now be thoroughly homogenized (Figure C-6) and ready to be used for electrode coating on the Draw Down Machine (DDM).

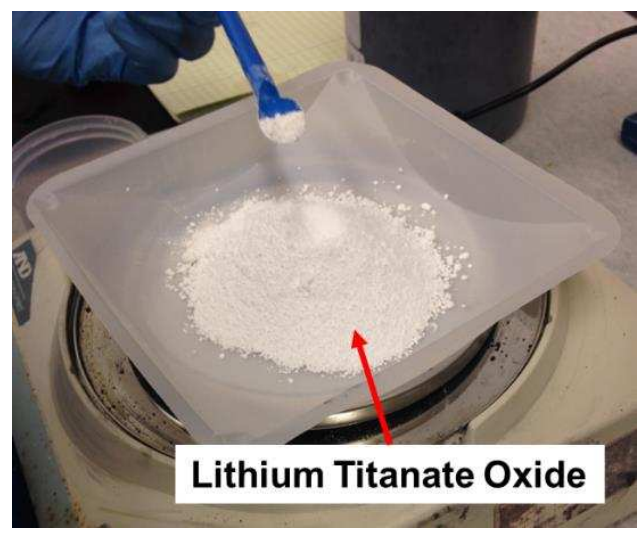

Figure C-4: Weighing Lithium Titanate Oxide Particles Prior to Inserting into Slurry Mixing Jar

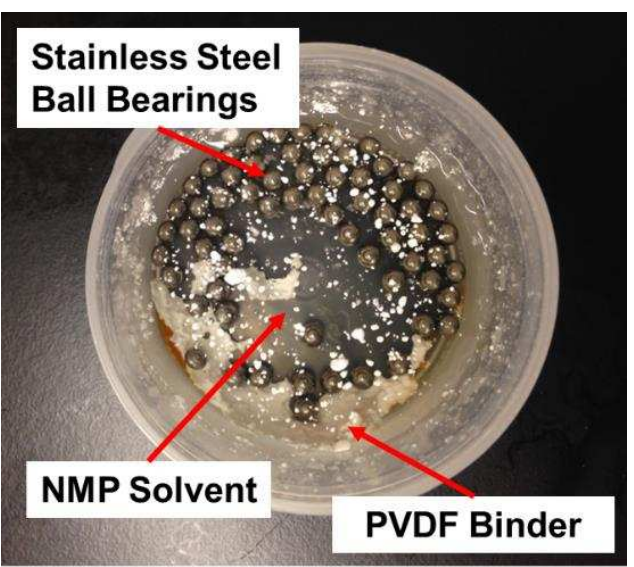

Figure C-2: NMP Solvent, PVDF Binder, and Stainless Steel Ball Bearings in Plastic Jar

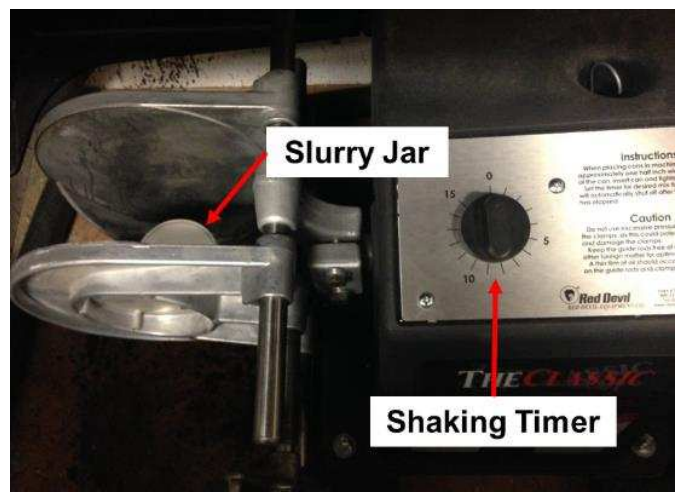

Figure C-3: Paint Can Shaker used for Slurry Homogenizing

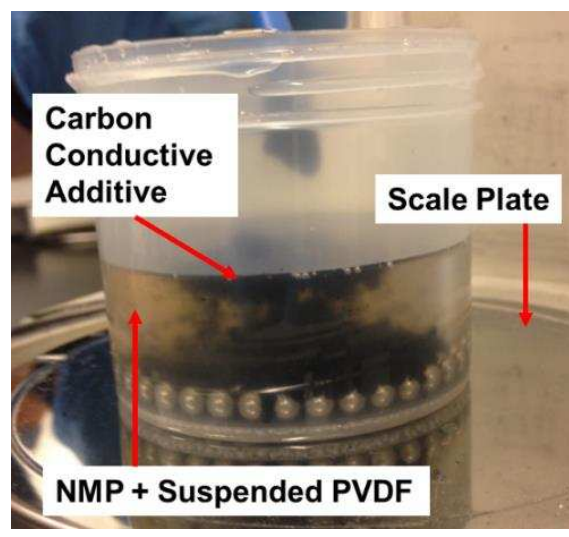

Figure C-5: NMP and Suspended PVDF with Carbon Conductive Additives 


\section{Current Collector (CC) Preparation on Draw Down Machine (DDM):}

1. Completely cover the coating surface of the DDM with a small amount of NMP to help the $\mathrm{CC}$ adhere to the surface.

2. Turn on the DMM's suction down feature to aid with holding the $\mathrm{CC}$ to the surface.

3. Apply a small amount of isopropanol (IPA) onto a Kimwipe and wipe the top surface of the CC. For aluminum, make sure the shiny (less oxidized) side is face-up (only if there is a visible difference between the two sides).

4. Using a steel wool roughing pad wetted with a little IPA, lightly scrub the top surface of the CC to roughen it. You should see scrape marks appear on the surface after properly scrubbing. This will help the slurry to adhere to the $\mathrm{CC}$ surface.

5. Use NMP on a Kimwipe to wipe the scraped surface clean. The CC is now ready for coating (Figure C-7).
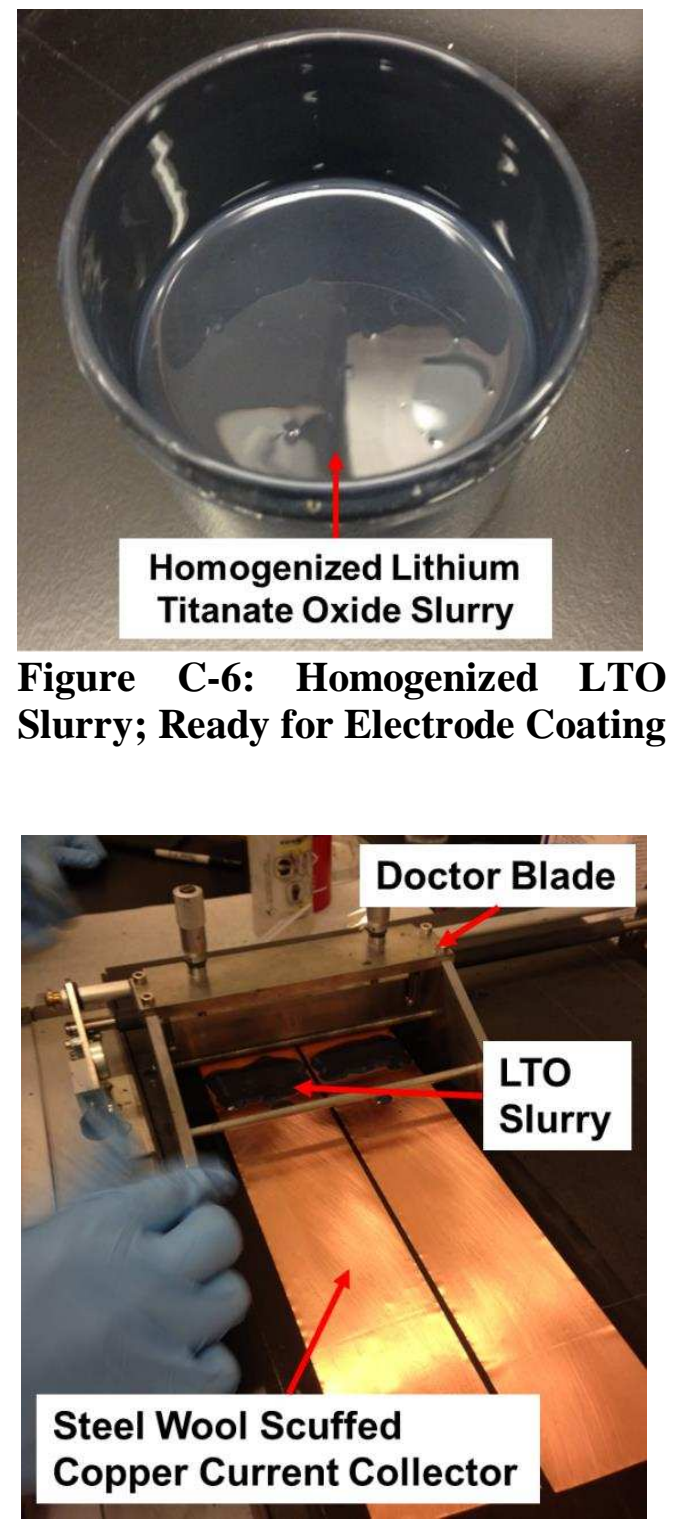

Figure C-7: Prepared Copper Current Collector with LTO Slurry Globs Prior to First Pass with DDM

\section{Coating the slurry onto the current collector using the DDM:}

1. Using a polypropylene spatula, glob the slurry onto the front of the CC strip ahead of the direction of travel (Figure C-8). The slurry glob will then be spread by the DDM which pushes the doctor blade over the glob to evenly spread it over the length of the CC strip. 
2. Set the doctor blade height. For the LTO and LFP electrodes coated in this study, the doctor blade height was set to $450 \mu \mathrm{m}$.

3. Place the doctor blade around the CC strips to be coated.

4. Press the $\rightarrow$ button on the DMM to advance the doctor blade over the length of the $\mathrm{CC}$ strip (Figure C-8).

5. Once complete press the $\leftarrow$ button to return the DDM to its home position.

6. Note where the coating stopped spreading on the CC strip. Glob additional slurry on areas that need

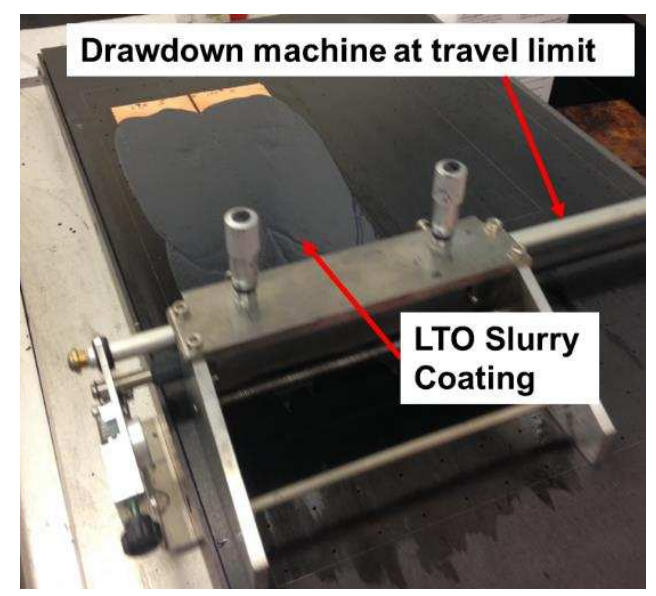

Figure C-8: Coated LTO Electrodes with DDM at Travel Limit to be coated more.

7. Repeat steps 3-6 until the entire CC is coated with electrode material minus the small portion at the top where the $\mathrm{CC}$ is labeled.

8. Remove the coated electrodes from the DDM using tweezers and hands covered with nitrile gloves. Be very careful moving the coated electrodes - the coating can very easily be wiped from the surface of the current collector.

9. Place the coated electrodes under a heat lamp for approximately 10 to 15 minutes (Figure C-9).

10. Transfer the coated electrodes to a vacuum oven.

11. Turn the vacuum oven on and set to $110^{\circ} \mathrm{C}$ for 1 hour. Do not draw vacuum on the chamber at this point.

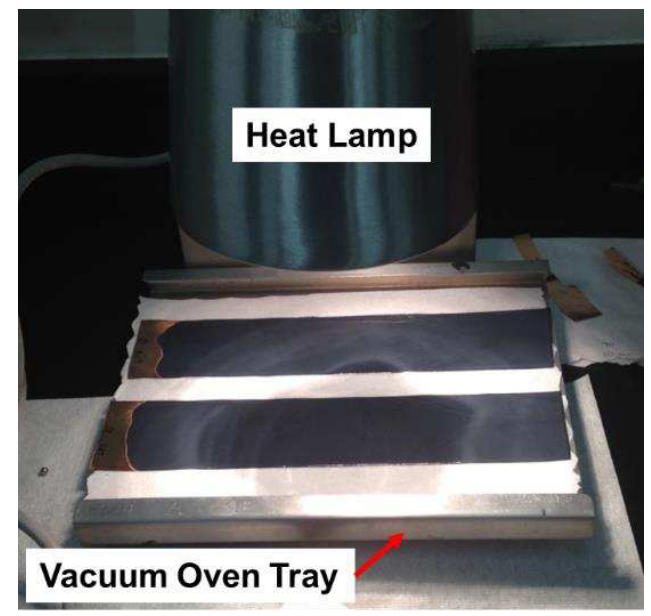

Figure C-9: Coated LTO Electrodes under Heat Lamp Prior to Vacuum Oven 
12. After 1 hour in the oven at atmospheric pressure, turn on the vacuum and let the electrodes vacuum dry overnight.

\section{Calendaring the Dried Electrodes:}

1. Using two sheets of nickel foil, sandwich the coated electrode strip (Figure C-10).

2. Feed the sandwich through the calendaring machine once. Check the thickness of the $\mathrm{CC}+$ coating using calipers.

3. The desired coating thickness is approximately $80 \mu \mathrm{m}$. Adjust

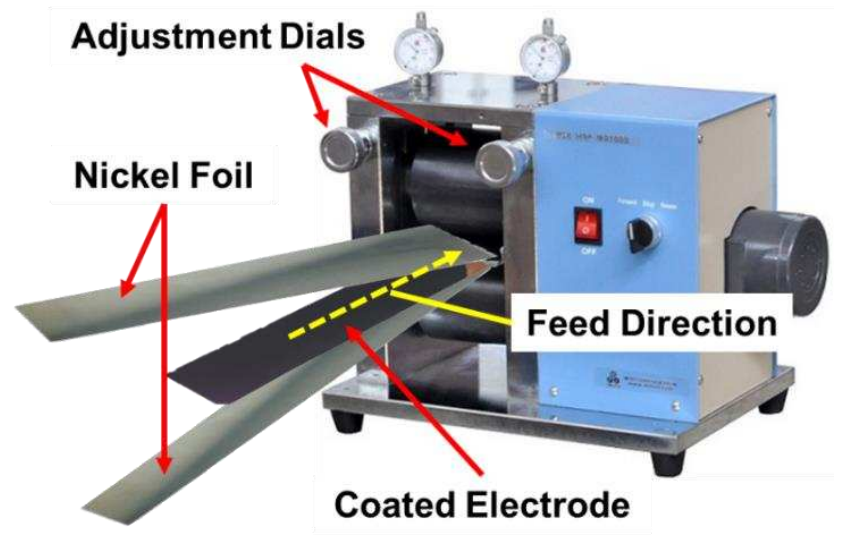
the dials on the front of the

Figure C-10: Calendaring Electrodes to Final Thickness

machine which set the roller height accordingly.

\section{Final Electrode Preparation:}

1. Measure the weight of the coated and calendared electrode strip. The difference in weight between the uncoated and coated current collector is the weight of the electrode coating.

2. Determine the average active material loading, $w_{\mathrm{AM}}$, of the $\mathrm{CC}$ strip in $\mathrm{mg} \mathrm{cm}^{-2}$ using the following formula:

$$
w_{\mathrm{AM}}=\frac{\left(m_{\mathrm{CE}}-m_{\mathrm{CC}}\right) \cdot Y_{\mathrm{AM}}}{A_{\mathrm{CC}}}
$$

where $m_{\mathrm{CE}}$ is the mass of the coated electrode strip, $m_{\mathrm{CC}}$ is the mass of the uncoated current collector strip, $Y_{\mathrm{AM}}$ is the mass fraction of active material in the electrode slurry, and $A_{\mathrm{CC}}$ is the coated area of the current collector strip. 
3. Using a $19 \mathrm{~mm}$ punch, create electrode disks that can be directly inserted into $19 \mathrm{~mm}$ PFA T-fitting.

4. Vacuum dry the electrodes at $80^{\circ} \mathrm{C}$ overnight before being assembled in a cell. 
Table D-1: Components of Electrolyte Boiling Facility

\begin{tabular}{|c|c|c|c|c|}
\hline Part & Description & Supplier & Part Number & $\begin{array}{l}\text { Electrolyte } \\
\text { Wetted? }\end{array}$ \\
\hline $\begin{array}{l}\text { Heater } \\
\text { Housing }\end{array}$ & $\begin{array}{l}\text { Machined Unfilled } \\
\text { PEEK }\end{array}$ & McMaster & 9089K129 & Yes \\
\hline $\begin{array}{l}\text { Heater } \\
\text { Sealing Plate }\end{array}$ & $\begin{array}{l}3003 \text { Aluminum, } \\
1.60 \mathrm{~mm} \text { Thick }\end{array}$ & McMaster & 8973K137 & Yes \\
\hline $\begin{array}{l}\text { Top \& } \\
\text { Bottom } \\
\text { Flanges }\end{array}$ & $\begin{array}{l}\text { Machined Unfilled } \\
\text { PEEK }\end{array}$ & McMaster & 9089K129 & Yes \\
\hline $\begin{array}{l}\text { Top \& } \\
\text { Bottom } \\
\text { Flange } \\
\text { Supports }\end{array}$ & $\begin{array}{l}\text { Machined Low } \\
\text { Carbon Steel, } 4.76 \\
\text { mm thick }\end{array}$ & McMaster & $1388 \mathrm{~K} 664$ & No \\
\hline $\begin{array}{l}\text { Teflon } \\
\text { Clamp }\end{array}$ & $\begin{array}{l}\text { PTFE Teflon, } 3.18 \\
\text { mm Thick }\end{array}$ & McMaster & $8735 \mathrm{~K} 14$ & Yes \\
\hline $\begin{array}{l}\text { Electrode } \\
\text { Stack } \\
\text { Clamping } \\
\text { Plate }\end{array}$ & $\begin{array}{l}\text { 316 Stainless Steel, } \\
2.67 \mathrm{~mm} \text { thick }\end{array}$ & McMaster & $88885 \mathrm{~K} 78$ & Yes \\
\hline Sight Glass & $\begin{array}{l}6.35 \mathrm{~cm} \text { OD Tube } \\
\text { Replacement Glass }\end{array}$ & MSC Direct & 61924767 & Yes \\
\hline Gaskets & $\begin{array}{l}\text { Pure PTFE Flange } \\
\text { Gasket, } 2 \text { Pipe Size, } \\
1.59 \mathrm{~mm} \text { thick }\end{array}$ & McMaster & $9483 \mathrm{~K} 846$ & Yes \\
\hline $\begin{array}{l}\text { Stainless } \\
\text { Steel Filler }\end{array}$ & $\begin{array}{l}\text { Machined } 316 \\
\text { Stainless Steel }\end{array}$ & McMaster & $89325 \mathrm{~K} 68$ & Yes \\
\hline O-Rings & $\begin{array}{l}\text { FF500-75 } \\
\text { Compound, Sizes } \\
\text { 2-011, 2-013, 2-029 }\end{array}$ & Parker & FF500-X-XXX & Yes \\
\hline $\begin{array}{l}\text { O-Ring } \\
\text { Sealing } \\
\text { Screw }\end{array}$ & $\begin{array}{l}8-32 \times 19 \mathrm{~mm}, \# 2 \\
\text { Drive, } 18-8 \text { Stainless, } \\
\text { Fluoroelastomer } \\
\text { O-Ring }\end{array}$ & McMaster & $90825 \mathrm{~A} 387$ & Yes \\
\hline $\begin{array}{l}\text { Heater } \\
\text { Housing } \\
\text { Clamping } \\
\text { Screws } \\
\end{array}$ & $\begin{array}{l}316 \text { SS Hex Head } \\
\text { Cap Screws } \\
6-32 \times 15.9 \mathrm{~mm}\end{array}$ & McMaster & 92185A149 & Yes \\
\hline
\end{tabular}




\begin{tabular}{|c|c|c|c|c|}
\hline Part & Description & Supplier & Part Number & $\begin{array}{l}\text { Electrolyte } \\
\text { Wetted? }\end{array}$ \\
\hline $\begin{array}{l}\text { Heater } \\
\text { Housing } \\
\text { Washers }\end{array}$ & $\begin{array}{l}\text { No. } 8 \text { lock washers, } \\
\text { general purpose } \\
\text { washers }\end{array}$ & McMaster & $\begin{array}{l}\text { 92147A420 } \\
90107 \mathrm{~A} 007\end{array}$ & Yes \\
\hline $\begin{array}{l}\text { Electrode } \\
\text { Stack } \\
\text { Clamping } \\
\text { Screws } \\
\end{array}$ & $\begin{array}{l}316 \text { SS Hex Head } \\
\text { Cap Screws } \\
8-32 \times 22.2 \mathrm{~mm}\end{array}$ & McMaster & 92185A198 & Yes \\
\hline $\begin{array}{l}\text { Electrode } \\
\text { Stack } \\
\text { Washers }\end{array}$ & $\begin{array}{l}\text { No. } 8 \text { lock washers, } \\
\text { general purpose } \\
\text { washers }\end{array}$ & McMaster & $\begin{array}{l}\text { 92147A425 } \\
90107 \mathrm{~A} 010\end{array}$ & Yes \\
\hline Tubing & $\begin{array}{l}316 \text { Stainless Steel } \\
\text { Smooth Bore Tubing }\end{array}$ & McMaster & $89785 \mathrm{~K} 823$ & Yes \\
\hline Tube Fittings & $\begin{array}{l}6.35 \mathrm{~mm} 316 \\
\text { Stainless Steel Tee, } \\
\text { Straight, Elbow } \\
\text { Fittings }\end{array}$ & Swagelok & $\begin{array}{l}\text { SS-400-3-4TTM } \\
\text { SS-400-3-4TMT } \\
\text { SS-400-1-4 } \\
\text { SS-400-9 }\end{array}$ & Yes \\
\hline $\begin{array}{l}\text { Condenser } \\
\text { Tube Fittings }\end{array}$ & $\begin{array}{l}316 \text { Stainless Steel } \\
\text { Tee and Straight } \\
\text { Fittings }\end{array}$ & Swagelok & $\begin{array}{l}\text { SS-810-3TFT } \\
\text { SS-810-3-4TTF } \\
\text { SS-400-1-6BT }\end{array}$ & No \\
\hline Ball Valves & $\begin{array}{l}6.35 \mathrm{~mm} \text { FNPT, } \\
\text { PTFE Seats }\end{array}$ & Swagelok & SS-83TF4 & Yes \\
\hline $\begin{array}{l}\text { Pre-Heater } \\
\text { Elbow } \\
\text { Fitting }\end{array}$ & $\begin{array}{l}316 \text { Stainless Steel } \\
\text { 7/16-20 ST Elbow } \\
\text { with O-Ring }\end{array}$ & Swagelok & SS-400-2-4ST & Yes \\
\hline $\begin{array}{l}\text { O-Ring Tube } \\
\text { Fittings for } \\
\text { PEEK } \\
\text { Flanges }\end{array}$ & $\begin{array}{l}316 \text { Stainless Steel, } \\
7 / 16-20 \text { ST }(6.35 \mathrm{~mm} \\
\text { Tube OD) or } 5 / 16-24 \\
\text { ST (1.59 mm Tube } \\
\text { OD) }\end{array}$ & Swagelok & $\begin{array}{l}\text { SS-400-1-OR } \\
\text { SS-400-1-ORBT } \\
\text { SS-100-1-ORBT }\end{array}$ & Yes \\
\hline $\begin{array}{l}\text { Pressure } \\
\text { Relief Safety } \\
\text { Valve }\end{array}$ & $\begin{array}{l}\text { 6.35 mm NPT Pop- } \\
\text { Safety Valve, } 172.4 \\
\mathrm{kPa}\end{array}$ & McMaster & $98905 \mathrm{~K} 15$ & Yes \\
\hline $\begin{array}{l}\text { NPT Cross } \\
\text { Fitting }\end{array}$ & $\begin{array}{l}\text { High-Pressure } 316 \\
\text { Stainless Steel, } 6.35 \\
\text { mm NPT }\end{array}$ & McMaster & 4443 K652 & Yes \\
\hline $\begin{array}{l}\text { NPT Tee } \\
\text { Fitting }\end{array}$ & $\begin{array}{l}\text { Extreme-Pressure } \\
316 \text { Stainless Steel, } \\
6.35 \mathrm{~mm} \text { NPT }\end{array}$ & McMaster & $51205 \mathrm{~K} 127$ & Yes \\
\hline $\begin{array}{l}\text { Barbed Hose } \\
\text { to NPT } \\
\text { Adapter }\end{array}$ & $\begin{array}{l}\text { 316 Stainless Steel, } \\
6.35 \mathrm{~mm} \text { Hose ID to } \\
6.35 \mathrm{~mm} \text { NPT }\end{array}$ & McMaster & $53505 \mathrm{~K} 64$ & Yes \\
\hline
\end{tabular}




\begin{tabular}{|c|c|c|c|c|}
\hline Part & Description & Supplier & Part Number & $\begin{array}{l}\text { Electrolyte } \\
\text { Wetted? }\end{array}$ \\
\hline $\begin{array}{l}\text { Heater } \\
\text { Thermo- } \\
\text { couples }\end{array}$ & $\begin{array}{l}\text { Type T Surface } \\
\text { Thermocouples, } \\
\text { Stripped Ends, } 3 \text { m }\end{array}$ & Omega & SA1XL-T-120 & No \\
\hline $\begin{array}{l}\text { Pre-Heater } \\
\text { Thermo- } \\
\text { couple }\end{array}$ & $\begin{array}{l}\text { PFA-insulated, Type } \\
\text { T, } 40 \text { gauge, stripped } \\
\text { lead termination }\end{array}$ & Omega & 5TC-TT-T-40-36 & No \\
\hline $\begin{array}{l}\text { Thin Film } \\
\text { Test Section } \\
\text { Heater }\end{array}$ & $\begin{array}{l}28 \mathrm{~V}, 10 \mathrm{~W} \text { Kapton } \\
\text { Insulated Flexible } \\
\text { Heater, } 0.254 \mathrm{~mm} \\
\text { Max Thickness, } 25.4 \\
\mathrm{~mm} \times 25.4 \mathrm{~mm}\end{array}$ & Omega & KHLV-101/10 & No \\
\hline $\begin{array}{l}\text { Thin Film } \\
\text { Pre-Heater }\end{array}$ & $\begin{array}{l}28 \mathrm{~V}, 10 \mathrm{~W} \text { Kapton } \\
\text { Insulated Flexible } \\
\text { Heater, } 0.254 \mathrm{~mm} \\
\text { Max Thickness, } 50.8 \\
\mathrm{~mm} \times 12.7 \mathrm{~mm}\end{array}$ & Omega & KHLV-0502/10 & No \\
\hline $\begin{array}{l}\text { Electrolyte } \\
\text { Bulk } \\
\text { Temperature } \\
\text { Probe }\end{array}$ & $\begin{array}{l}\text { Quick Disconnect T- } \\
\text { Type Thermocouple, } \\
316 \text { Stainless Steel }\end{array}$ & Omega & $\begin{array}{l}\text { CP316SS-116U- } \\
12\end{array}$ & Yes \\
\hline $\begin{array}{l}\text { Condenser } \\
\text { Thermo- } \\
\text { couples }\end{array}$ & $\begin{array}{l}6.35 \mathrm{~mm} \text { NPT T- } \\
\text { Type Thermocouples }\end{array}$ & Omega & TC-T-NPT-U-72 & No \\
\hline $\begin{array}{l}\text { Vapor } \\
\text { Pressure } \\
\text { Transmitter }\end{array}$ & $\begin{array}{l}\text { 4-20 mA Output, 0- } \\
\text { 344.7 kPa, } 316 \mathrm{SS} \\
\text { Wetted Parts, } \\
6.35 \mathrm{~mm} \text { NPT }\end{array}$ & Omega & PX309-050AI & Yes \\
\hline $\begin{array}{l}\text { Vapor } \\
\text { Pressure } \\
\text { Transmitter } \\
\text { Power } \\
\text { Supply } \\
\end{array}$ & $\begin{array}{l}\text { AC-DC Power } \\
\text { Supply, } 26 \mathrm{~V} \text { at } 1.3 \\
\text { A, 85-264 V In }\end{array}$ & $\begin{array}{l}\text { Allied } \\
\text { Electronics }\end{array}$ & $\begin{array}{l}\text { SOLA-HD } \\
\text { SDP1-24-100T }\end{array}$ & No \\
\hline $\begin{array}{l}\text { Power } \\
\text { Supply Fuse } \\
\text { to NI } 9207\end{array}$ & $\begin{array}{l}2 \text { A maximum fast- } \\
\text { acting fuse }\end{array}$ & $\begin{array}{l}\text { Mountain } \\
\text { States } \\
\text { Electronics } \\
\end{array}$ & N/A & No \\
\hline Wire & $\begin{array}{l}18 \text { Gauge, Red \& } \\
\text { Black }\end{array}$ & $\begin{array}{l}\text { Mountain } \\
\text { States } \\
\text { Electronics }\end{array}$ & N/A & No \\
\hline Bread Board & Classic style & $\begin{array}{l}\text { Mountain } \\
\text { States } \\
\text { Electronics }\end{array}$ & N/A & No \\
\hline
\end{tabular}




\begin{tabular}{|c|c|c|c|c|}
\hline Part & Description & Supplier & Part Number & $\begin{array}{l}\text { Electrolyte } \\
\text { Wetted? }\end{array}$ \\
\hline $\begin{array}{l}\text { Alligator } \\
\text { Clips }\end{array}$ & $\begin{array}{l}304 \text { Stainless Steel } \\
\text { Micro Alligator Clip, } \\
\text { 5A }\end{array}$ & Digi-Key & 314-1017-ND & Yes \\
\hline $\begin{array}{l}\text { Resistor for } \\
\text { DAQ } \\
\text { Voltage } \\
\text { Sense } \\
\end{array}$ & $1 \mathrm{M} \Omega$ resistance & $\begin{array}{l}\text { SparkFun } \\
\text { Electronics }\end{array}$ & COM-10969 & No \\
\hline $\begin{array}{l}\text { Thermo- } \\
\text { couple Data } \\
\text { Acquisition } \\
\text { Card }\end{array}$ & $\begin{array}{l}\text { 16-Channel } \\
\text { Isothermal } \\
\text { Thermocouple Input } \\
\text { Module }\end{array}$ & $\begin{array}{l}\text { National } \\
\text { Instruments }\end{array}$ & NI 9214 & No \\
\hline $\begin{array}{l}\text { Current \& } \\
\text { Voltage Data } \\
\text { Acquisition } \\
\text { Card }\end{array}$ & $\begin{array}{l}\text { Voltage/Current } \\
\text { Analog Input, } 500 \\
\text { Samples/second, } 16 \\
\text { Channel Module }\end{array}$ & $\begin{array}{l}\text { National } \\
\text { Instruments }\end{array}$ & $\begin{array}{l}\text { NI } 9207 \text { with } \\
\text { D-SUB }\end{array}$ & No \\
\hline $\begin{array}{l}\text { Data } \\
\text { Acquisition } \\
\text { Card Chassis }\end{array}$ & $\begin{array}{l}\text { CompactDAQ 4-Slot } \\
\text { USB Chassis }\end{array}$ & $\begin{array}{l}\text { National } \\
\text { Instruments }\end{array}$ & cDAQ-9174 & No \\
\hline $\begin{array}{l}\text { Compression } \\
\text { Threaded } \\
\text { Rods }\end{array}$ & $\begin{array}{l}316 \text { Stainless Steel } \\
1 / 4-20 \times 20.3 \mathrm{~cm}\end{array}$ & McMaster & 90575 A223 & No \\
\hline $\begin{array}{l}\text { Nuts for } \\
\text { Compression } \\
\text { Rod }\end{array}$ & $\begin{array}{l}\text { Hex Nut, } \\
\text { High-Torque 12- } \\
\text { Point Flange Nut }\end{array}$ & McMaster & $\begin{array}{l}\text { 94819A043 } \\
\text { 90759A100 }\end{array}$ & No \\
\hline $\begin{array}{l}\text { Washers for } \\
\text { Compression } \\
\text { Rod }\end{array}$ & $\begin{array}{l}\text { Black-Oxide Steel } \\
\text { Oversized Flat, Split } \\
\text { Lock }\end{array}$ & McMaster & $\begin{array}{l}\text { 90377A } 157 \\
91475 \mathrm{~A} 029\end{array}$ & No \\
\hline $\begin{array}{l}\text { Standoff } \\
\text { Nuts }\end{array}$ & $\begin{array}{l}\text { Nylon } 6 / 6 \text { Female } \\
\text { Threaded Standoff, } \\
25.4 \mathrm{~mm} \text { Length, 1/4- } \\
20\end{array}$ & McMaster & 92319A550 & No \\
\hline $\begin{array}{l}\text { Tubing } \\
\text { Support } \\
\text { Clamps }\end{array}$ & $\begin{array}{l}\text { Vibration-Damping } \\
\text { Loop Clamps, } 3.175 \\
\text { cm ID }\end{array}$ & McMaster & $3225 \mathrm{~T} 61$ & No \\
\hline $\begin{array}{l}\text { Electrolyte } \\
\text { Mixing \& } \\
\text { Charging Jar }\end{array}$ & $\begin{array}{l}180 \text { mL Glass Jar, } \\
\text { Phenolic Cap with } \\
\text { PTFE Seal }\end{array}$ & McMaster & 4239T33 & Yes \\
\hline $\begin{array}{l}\text { Check Valve } \\
\text { for Charging } \\
\text { Jar }\end{array}$ & $\begin{array}{l}\text { Miniature Check } \\
\text { Valve, } 303 \text { SS }\end{array}$ & McMaster & $8567 \mathrm{~T} 32$ & Yes \\
\hline
\end{tabular}


Electrolyte

\begin{tabular}{|c|c|c|c|c|}
\hline Part & Description & Supplier & Part Number & Wetted? \\
\hline $\begin{array}{l}\text { Teflon } \\
\text { Tubing for } \\
\text { Electrolyte } \\
\text { Charging }\end{array}$ & $\begin{array}{l}\text { Teflon PTFE, } 6.35 \\
\text { mm ID, } 7.94 \mathrm{~mm} \\
\text { OD, Semi-Clear } \\
\text { White }\end{array}$ & McMaster & $5239 \mathrm{~K} 13$ & Yes \\
\hline $\begin{array}{l}\text { Epoxy for } \\
\text { Charging Jar } \\
\text { Lid }\end{array}$ & $\begin{array}{l}\text { WEST System G/flex } \\
650-8 \text { Liquid Epoxy }\end{array}$ & $\begin{array}{l}\text { Jamestown } \\
\text { Distributors }\end{array}$ & WSY-650-8 & Yes \\
\hline
\end{tabular}




\section{APPENDIX E. THERMOCOUPLE CALIBRATION}

All of the T-type thermocouples used in the electrolyte boiling facility were calibrated using a Fluke 5615 platinum resistance device with a Fluke 1502A readout. The internal standard has a NIST-traceable calibrated uncertainty of $\pm 0.012^{\circ} \mathrm{C}$. A water calibration bath was created by using Chemglass AREC.X Heating Magnetic Stirring Plate (CG-1999-V-10) with an insulated glass beaker. A magnetic stirring bar was inserted into the bottom of the beaker to continually circulate the water bath. The heated stirring plate contains a PT100 temperature probe that provides temperature feedback to control the water temperature to within $\pm 1.0^{\circ} \mathrm{C}$. All surface thermocouples were wrapped into a plastic bag prior to insertion into the water bath. The 5615 temperature probe and the thermocouples were fixtured above the glass beaker using ring stand clamps. The top of the water bath was then covered with insulation. The temperature from the Fluke 1502A readout and the thermocouple measurements was recorded using a LabVIEW VI. The thermocouples were calibrated over a nominal range of $0^{\circ} \mathrm{C}$ to $90^{\circ} \mathrm{C}$ with seven set points $\left(0,25,40,50,60,75,90^{\circ} \mathrm{C}\right)$. This temperature range encompassed the expected temperature range for the present study. At each temperature set point, a minimum of 50 temperature measurements were made over 50 seconds.

After recording the thermocouple and 5615 temperature data at each set point, a linear regression was fit to the average of each thermocouple's measured value versus the average of the 5616 standard measurement. The resulting slope and intercept was used as the calibration fit for each thermocouple. A calibration uncertainty was determined for each of the thermocouples which is a combination of the bias uncertainty of the Fluke standard and precision uncertainty of the thermocouple measurement. The following equations were used to determine the total measurement uncertainty of each of the thermocouples. 
To determine the bias uncertainty associated with the calibration, the standard error of estimate (SEE) was calculated as follows:

$$
S E E^{2}=\frac{\sum_{i=1}^{N}\left[Y_{i}-\left(a X_{i}+b\right)\right]^{2}}{N-2}
$$

where $Y_{i}$ is the average temperature measured by the 5615 standard at the temperature set point, $a$ is the slope of the linear regression, $X_{i}$ is the average temperature measured by the thermocouple at the temperature set point, $b$ is the intercept of the linear regression, and $N$ is the number of calibration set points. For the Heater TC 1 thermocouple, the slope (1.00) and intercept (-0.28) of the calibration fit gave a $S E E^{2}$ of $0.020\left[{ }^{\circ} \mathrm{C}^{2}\right]$. The total bias uncertainty of the thermocouple calibration was calculated using:

$$
B_{\mathrm{TC}}=\sqrt{B_{\mathrm{TS}}{ }^{2}+4 S E E^{2}}
$$

where $B_{\mathrm{TS}}$ is the bias uncertainty of the 5615 temperature calibration standard $\left(0.012^{\circ} \mathrm{C}\right)$. For the Heater TC 1 thermocouple, the bias uncertainty was determined to be $0.28^{\circ} \mathrm{C}$.

To determine the precision uncertainty, which is the random error associated with a measured value of the thermocouple, several parameters were calculated. First, the sample mean $(\bar{X})$ and standard deviation $\left(S_{\mathrm{X}}\right)$ of the thermocouple measurement at each of the set points were determined as follows:

$$
\begin{gathered}
\bar{X}=\frac{1}{N} \sum_{i=1}^{N} X_{i} \\
S_{\mathrm{X}}=\left[\frac{1}{N-1} \sum_{i=1}^{N}\left(X_{i}-\bar{X}\right)^{2}\right]^{1 / 2}
\end{gathered}
$$


The precision error $\left(P_{\mathrm{X}}\right)$ at each temperature set point can then be estimated using a $t$-distribution with $N$-1 degrees of freedom, where $N$ is the number of temperature measurements collected at each set point:

$$
P_{\mathrm{X}}=t \frac{S_{\mathrm{X}}}{\sqrt{N}}
$$

For a $95 \%$ confidence interval and 50 temperature measurements at a single temperature set point $(N), t$ is conservatively estimated to be 2.021 (the closest value of $t$ provided was for 40 degrees of freedom). Values of $t$ can be found in a $t$-distribution lookup table for various degrees of freedom and confidence intervals. The precision error at each temperature set point was calculated and then averaged to determine the total precision uncertainty of the thermocouple $\left(\bar{P}_{\mathrm{X}}\right)$ :

$$
\bar{P}_{\mathrm{X}}=\frac{1}{N} \sum_{i=1}^{N} P_{\mathrm{X}}
$$

For the Heater TC 1 thermocouple, the total precision uncertainty was determined to be $0.0045^{\circ} \mathrm{C}$.

Lastly, the total uncertainty which combines the bias uncertainty of the calibration with the precision uncertainty of the measurement was determined using:

$$
U_{\mathrm{T}}=\sqrt{B_{\mathrm{TC}}^{2}+\bar{P}_{\mathrm{X}}^{2}}
$$

For the Heater TC 1 thermocouple, the bias uncertainty of $0.28^{\circ} \mathrm{C}$ and precision uncertainty of $0.0045^{\circ} \mathrm{C}$ gave a total uncertainty of $0.28^{\circ} \mathrm{C}$. The summary of calibration and uncertainty calculations for each of the thermocouples used in the electrolyte boiling facility is provided in Table E-1. 
Table E-1: Thermocouple Calibration Fits and Total Uncertainty

\begin{tabular}{lllllll}
\multicolumn{1}{c}{ Thermocouple ID } & $\begin{array}{l}\text { Heater } \\
\text { TC 1 }\end{array}$ & $\begin{array}{l}\text { Heater } \\
\text { TC 2 }\end{array}$ & $\begin{array}{l}\text { Heater } \\
\text { TC 3 }\end{array}$ & $\begin{array}{l}\text { Probe } \\
\text { TC }\end{array}$ & $\begin{array}{l}\text { NPT } \\
\text { TC 1 }\end{array}$ & $\begin{array}{l}\text { NPT } \\
\text { TC 2 }\end{array}$ \\
\hline Linear Regression Slope, $\boldsymbol{a}$ & 1.00 & 1.00 & 1.00 & 0.99 & 1.00 & 1.00 \\
\hline Linear Regression Intercept, $\boldsymbol{b}$ & -0.28 & -0.29 & -0.27 & -0.07 & -0.08 & -0.16 \\
\hline Standard Error of Estimate, $\boldsymbol{S E E}$ & 0.020 & 0.00038 & 0.00076 & 0.00023 & 0.00048 & 0.0027 \\
\hline Set Point Measurement Count, $\boldsymbol{N}$ & 50 & 50 & 50 & 50 & 50 & 50 \\
\hline Bias (standard), $\boldsymbol{B}_{\mathbf{T s}}\left[{ }^{\circ} \mathbf{C}\right]$ & 0.012 & 0.012 & 0.012 & 0.012 & 0.012 & 0.012 \\
\hline Bias Uncertainty, $\boldsymbol{B}_{\mathbf{T C}}\left[{ }^{\circ} \mathbf{C}\right]$ & 0.28 & 0.041 & 0.056 & 0.033 & 0.046 & 0.11 \\
\hline Precision Uncertainty,,$\overline{\boldsymbol{P}}_{\mathbf{X}}\left[{ }^{\circ} \mathbf{C}\right]$ & 0.0045 & 0.0055 & 0.0057 & 0.010 & 0.0058 & 0.0061 \\
\hline Total Uncertainty, $\boldsymbol{U}_{\mathbf{T}}\left[{ }^{\circ} \mathbf{C}\right]$ & 0.280 & 0.041 & 0.057 & 0.034 & 0.046 & 0.105
\end{tabular}

